\title{
Quiver gauge theories: beyond reflexivity
}

\author{
Jiakang Bao, ${ }^{a}$ Grace Beaney Colverd $^{b}$ and Yang-Hui He $\mathrm{He}^{a, b, c}$ \\ ${ }^{a}$ Department of Mathematics, City University of London, \\ Northampton Square, London, EC1V OHB, U.K. \\ ${ }^{b}$ Department of Physics, University of Oxford, \\ Parks Road, Oxford, OX1 3PU, U.K. \\ ${ }^{c}$ School of Physics, NanKai University, \\ 94 Weijin Road, Tianjin, 300071, P.R. China \\ E-mail: jiakang.bao@city.ac.uk, grace.bcolverd@gmail.com, \\ hey@maths.ox.ac.uk
}

ABSTRACT: Reflexive polygons have been extensively studied in a variety of contexts in mathematics and physics. We generalize this programme by looking at the 45 different lattice polygons with two interior points up to $\mathrm{SL}(2, \mathbb{Z})$ equivalence. Each corresponds to some affine toric 3 -fold as a cone over a Sasaki-Einstein 5 -fold. We study the quiver gauge theories of D3-branes probing these cones, which coincide with the mesonic moduli space. The minimum of the volume function of the Sasaki-Einstein base manifold plays an important role in computing the R-charges. We analyze these minimized volumes with respect to the topological quantities of the compact surfaces constructed from the polygons. Unlike reflexive polytopes, one can have two fans from the two interior points, and hence give rise to two smooth varieties after complete resolutions, leading to an interesting pair of closely related geometries and gauge theories.

Keywords: Brane Dynamics in Gauge Theories, Differential and Algebraic Geometry, Duality in Gauge Field Theories

ArXiv EPrint: 2004.05295 


\section{Contents}

1 Introduction 1

2 Quiver gauge theories and the inverse algorithm 4

2.1 Lattice polytopes 4

2.2 Brane tilings 5

2.3 The moduli spaces 8

2.4 Volume minimization 9

3 Five triangles $\quad 12$

3.1 Polytope $1: \mathbb{C}^{3} / \mathbb{Z}_{6}(1,1,4) \quad 12$

3.2 Polytope $2: \mathbb{C}^{3} / \mathbb{Z}_{5}(1,2,2) \quad 14$

$\begin{array}{lll}3.3 & \text { Polytope } 3: \mathbb{C}^{3} / \mathbb{Z}_{8}(1,3,4) & 16\end{array}$

3.4 Polytope 4: $\mathbb{C}^{3} /\left(\mathbb{Z}_{2} \times \mathbb{Z}_{5}\right)(1,0,1)(0,1,4) \quad 18$

3.5 Polytope $5: \mathbb{C}^{3} /\left(\mathbb{Z}_{2} \times \mathbb{Z}_{6}\right)(1,0,1)(1,0,5)$

4 Nineteen quadrilaterals $\quad \mathbf{2 5}$

4.1 Polytope 6: $L^{3,3,1} \quad 25$

$\begin{array}{lll}4.2 & \text { Polytope } 7: L^{3,3,2} & 27\end{array}$

$\begin{array}{lll}4.3 & \text { Polytope 8: } Y^{3,0} & 29\end{array}$

4.4 Polytope 9: $\mathrm{SPP} /\left(\mathbb{Z}_{2} \times \mathbb{Z}_{2}\right)(1,0,0,1)(0,1,1,0) \quad 30$

4.5 Polytope 10: $L^{2,3,2} / \mathbb{Z}_{2}(1,0,0,1) \quad 32$

4.6 Polytope 11: $\mathrm{dP}_{1} / \mathbb{Z}_{2}(1,0,0,1) \quad 36$

4.7 Polytope $12: L^{1,4,1} / \mathbb{Z}_{2}(1,0,0,1) \quad 38$

4.8 Polytope 13: $\mathrm{PdP}_{2} / \mathbb{Z}_{2}(1,1,1,1) \quad 40$

4.9 Polytope 14: $L^{1,3,1} / \mathbb{Z}_{2}(1,0,0,1)$

4.10 Polytope 15: $L^{3,5,2} 44$

4.11 Polytope $16 ; L^{2,5,1} 46$

4.12 Polytope 17: $L^{5,6,1} 48$

4.13 Polytope 18: $L^{2,4,1} \quad 50$

4.14 Polytope 19: $L^{5,4,1} \quad 52$

4.15 Polytope 20: $L^{1,5,1} / \mathbb{Z}_{2}(1,0,0,1) \quad 53$

4.16 Polytope 21: $\mathrm{SPP} / \mathbb{Z}_{3}(1,0,0,2) \quad 55$

4.17 Polytope 22: $\mathcal{C} /\left(\mathbb{Z}_{3} \times \mathbb{Z}_{2}\right)(1,0,0,2)(0,1,1,0) \quad 56$

4.18 Polytope 23: $L^{1,3,2} \quad 57$

$\begin{array}{ll}4.19 \text { Polytope } 24: \mathcal{C} / \mathbb{Z}_{4}(0,1,2,1) & 59\end{array}$

$\begin{array}{lll}5 & \text { Sixteen pentagons } & \mathbf{6 1}\end{array}$

5.1 Polytope 25: $X^{3,2}-62$

5.2 Polytope 26: $X^{3,1} \quad 64$

5.3 Polytope 27: $\mathrm{PdP}_{4 c}(2) \quad 66$ 
5.4 Polytope 28: $\mathrm{PdP}_{4 d}(2) \quad 68$

5.5 Polytope 29: $\mathrm{PdP}_{5 b}(2) \quad 70$

5.6 Polytope 30: $\mathrm{PdP}_{6 a}(2) \quad 72$

5.7 Polytope 31: $K^{2,5,1,4}-73$

5.8 Polytope 32: $K^{2,5,1,3}-75$

5.9 Polytope 33: $K^{2,5,1,2} \quad 76$

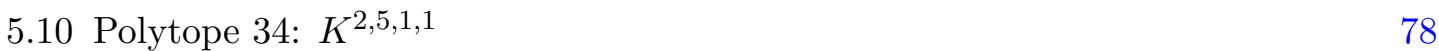

5.11 Polytope 35: $K^{4,4,2,4} \quad 80$

5.12 Polytope $36: K^{4,4,2,2} \quad 82$

5.13 Polytope 37: $K^{2,4,1,3} \quad 83$

5.14 Polytope 38: $K^{2,4,1,2} 85$

5.15 Polytope 39: $K^{2,4,1,1} \quad 87$

5.16 Polytope 40: $K^{4,3,2,2} 89$

6 Five hexagons $\quad 91$

6.1 Polytope 41: $\mathrm{PdP}_{4 e}(3) \quad 91$

6.2 Polytope 42: $\operatorname{PdP}_{5 c}(3) \quad 93$

6.3 Polytope 43: $\mathrm{PdP}_{6 b}(3) \quad 95$

6.4 Polytope 44: $\mathrm{PdP}_{4 f}(2) \quad 97$

6.5 Polytope 45: $\operatorname{PdP}_{6 c}(3) \quad 99$

7 The toric variety $\widetilde{X(\Delta)} \quad 101$

7.1 The two interior points as origins 102

7.2 Minimized volumes and topological quantities 105

8 Conclusions and outlook 110

A The 45 lattice polygons with two interior points 112

B Volume functions $r 13$

$\begin{array}{lr}\text { C Higgsing the parent theory } & 115\end{array}$

$\begin{array}{ll}\text { D More toric phases } & 116\end{array}$

\section{Introduction}

The worldvolume theory of a stack of D3-branes probing a toric Calabi-Yau (CY) conetype singularity is a $4 \mathrm{~d} \mathcal{N}=1$ supersymmetric gauge theory. Such gauge theories can be represented by quivers in which the bifundamental matter contents and the superpotentials are encoded ${ }^{1}$ [1]. Each toric $\mathrm{CY}_{3}$ corresponds to a toric diagram which is a 2-dimensional

\footnotetext{
${ }^{1}$ Saying this, we should bear in mind that the superpotential is generally additional data for defining a theory, unless we are considering periodic quivers for toric theories.
} 


\begin{tabular}{|c|cccccccccc|}
\hline & 0 & 1 & 2 & 3 & 4 & 5 & 6 & 7 & 8 & 9 \\
\hline D5 & $\times$ & $\times$ & $\times$ & $\times$ & & $\times$ & & $\times$ & & \\
\hline NS5 & $\times$ & $\times$ & $\times$ & $\times$ & - & & & & & \\
\hline
\end{tabular}

Table 1. The brane configuration of D5 and NS5 branes.

lattice polytope, viz, a lattice polygon. The geometry of the $\mathrm{CY}_{3}$ 's can thus be studied via their toric diagrams.

Hence, it is natural to expect that there are some connections between the quivers and toric diagrams. From one diagram, we can find the other following the approaches in $[2,3]$. Given a quiver diagram, the process of finding the toric diagram is called the forward algorithm. Conversely, obtaining quivers from a toric diagram is known as the inverse algorithm. Generally speaking, the correspondence between the two kinds of diagrams is often one-to-many. A toric diagram may give rise to more than one quivers while many quivers can have the same toric diagram. As a matter of fact, these quiver theories are related by toric duality, which can be understood as Seiberg duality in the toric phases [1,4].

If we consider the back reaction to the geometry from D3s, then we get an AdS nearhorizon geometry. As a result, the gauge/gravity duality [5] gives another point of view to the above problem. The $4 \mathrm{~d} \mathcal{N}=4 \mathrm{SYM}$ theory is related to the string theory in $\operatorname{AdS} \times S^{5}$. If we replace the 5 -sphere with a Sasaki-Einstein manifold $Y$ of real dimension 5, then the SUSY is broken down to $\mathcal{N}=1[6,7]$.

In fact, we can use type IIB brane configurations to study this. Consider D5-branes suspended between an NS5-brane wrapping a holomorphic surface $\Sigma$ as tabulated in table 1 . Then the Newton polynomial of the toric diagram defines this holomorphic surface. The system is compactified along directions 5 and 7 on a torus $\mathbb{T}^{2}$. After performing a T-duality on each of these two directions, the D5s would be mapped back to D3s probing the CY 3 -fold.

We can draw a 5-brane web diagram on $\mathbb{T}^{2}$. The dual graph of the web diagram is then a bipartite periodic graph on the torus. Such dual graphs are known as dimers/brane tilings [8-12]. With the help of brane tilings, we are able to bridge the toric diagrams and the quivers.

Similar stories also happen in other dimensions. Under $n$ T-dualities, the system of $\mathrm{D}(7-n)$-branes suspended between an NS5 wrapping a holomorphic $n$-cycle, where the branes meet in a $\mathbb{T}^{n}$, corresponds to $\mathrm{D}(7-2 n)$-branes probing $\mathrm{CY}_{n+1}$ [13]. These are related to various topics in different dimensions, such as Chern-Simons theory [14-18], brane brick models [19-22], triality [23], quadrality [24] and so forth.

For reflexive polytopes, the cases are very well-studied in $[13,25,26]$. In this paper, we will try to extend these to non-reflexive cases, in particular, polygons with two interior points. Up to $\mathrm{SL}(2, \mathbb{Z})$ equivalence, there are 45 such polygons (5 triangles, 19 quadrilaterals, 16 pentagons and 5 hexagons). They are found in [27] and we list them in appendix A, as well as their volume functions in appendix B. Hence, we will apply the inverse algorithm to get the corresponding gauge theories. Most of the toric varieties are related to known 
families including $\mathbb{C}^{3}$, (generalized) conifolds $(\mathcal{C})[28,29]$, suspended pinch point (SPP), $Y^{p, q}[30-33], L^{a, b, c}[34], X^{p, q}[35]$ and (pseudo) del Pezzos ((P)dP) [2, 36-38]. When orbifolding a space, the orbifold action can be determined via Hermite normal forms and barycentric coordinates $[39,40]$. In particular, some of the quivers and superpotentials are studied in previous literature, such as $Y^{3,0}$ in [41] and toric diagrams up to (normalized) area 8 in [42]. In [43-45], some of the toric diagrams are studied from 5d SCFT perspective. The number of interior points is the rank of the 5d SCFT, which sheds light onto the classification of 5d SCFTs.

We start by briefly reviewing the relevant background of quivers and volume minimizations in section 2. Then in section 3-6, we report the gauge theories obtained from inverse algorithm. Since many toric diagrams correspond to a large number of quivers, we will present only one toric quiver for each polytope. Some more toric quivers in different phases are presented in appendix D. In section 7 , we will turn to the compact surfaces constructed from these toric diagrams. The relevant topology can be related to the volume minization which plays an important role especially in R-symmetry. Finally, we will make a summary and discuss possible future directions in section 8 .

\section{Nomenclature}

$X(\Delta)$

$\widetilde{X}=\widetilde{X(\Delta)}$

$X_{i}(\Delta)$

$\widetilde{X_{1}^{\prime}(\Delta)}$

$\mathcal{X}$

$\mathcal{M} / \Gamma\left(a_{1}, \ldots, a_{n}\right) \quad$ : orbifold from space $\mathcal{M}$ quotiented by a discrete group $\Gamma$

with group action $\left(z_{1}, z_{2}, z_{3}\right) \sim\left(\omega z_{1}, \omega z_{2}, \omega^{4} z_{3}\right)$ where $\omega^{6}=1$

$H S=H S\left(t_{i} ; \mathcal{X}\right) \quad: \quad$ Hilbert series of $\mathcal{X}$, with $t_{i}$ being the fugacities

$Y=\mathcal{B}_{\mathbb{R}}(\mathcal{X})$ with action $\left(a_{1}, \ldots, a_{n}\right)$

e.g. orbifold $\mathbb{C}^{3} / \mathbb{Z}_{6}(1,1,4)$ is $\mathbb{C}^{3}$ quotiented by $\mathbb{Z}_{6}$

: convex lattice polytope

: compact toric variety corresponding to $\Delta$;

if $\Delta \subset \mathbb{Z}^{n}$, then $X(\Delta)$ is of complex dimension $n$

: complete resolution of $X(\Delta)$

: compact toric variety;

obtained from choosing the $i^{\text {th }}$-grade point as the origin

: toric variety $\widetilde{X_{1}(\Delta)}$ with a further blow-up

: $\quad$ affine $\mathrm{CY}$ cone over $\Delta$;

if $\Delta \subset \mathbb{Z}^{n}$, then $X(\Delta)$ is of complex dimension $(n+1)$

Sasaki-Einstein base manifold of $\mathcal{X}$;

if $\operatorname{dim}_{\mathbb{C}}(\mathcal{X})=n+1$, then $Y$ is of real dimension $(2 n+1)$ 


$$
\begin{array}{ll}
V=V\left(b_{i} ; Y\right) & : \text { volume function of } Y, \text { with } b_{i} \text { being components of Reeb vector } \\
I \& N_{P} & : \text { number of interior \& perimeter points of the toric diagram } \\
G & : \text { number of gauge nodes in the quiver } \\
E & : \text { number of bifundamentals in the quiver } \\
c & : \text { structure sheaf of } \widetilde{\mathcal{X}} \\
\mathcal{O}_{\tilde{\mathcal{X}}} & : \text { (Weil) divisor } D \text { on } \widetilde{\mathcal{X}} \\
D & : \text { sheaf of divisor } D \text { on } \widetilde{\mathcal{X}} \\
\mathcal{O}_{\tilde{\mathcal{X}}}(D) & : \text { (total) Chern class of } \widetilde{\mathcal{X}}, \text { with } c_{i} \text { denoting the } i^{\text {th }} \text { Chern class } \\
c(\widetilde{\mathcal{X}}) & : \text { the } i^{\text {th }} \text { Chern number } \\
C_{i} & \text { (the top Chern number is the Euler number } \chi)
\end{array}
$$

\section{Quiver gauge theories and the inverse algorithm}

We begin with a lightning review of the key requisite concepts, from toric CY cones to quiver gauge theories.

\subsection{Lattice polytopes}

A lattice polytope $\Delta$ is a convex hull of a finite number of points in $\mathbb{Z}^{n}$, and its vertices form the set $\Delta \cap \mathbb{Z}^{n}$. A polytope is said to be reflexive if its dual polyotpe

$$
\Delta^{\circ}=\left\{\boldsymbol{v} \in \mathbb{Z}^{n}: \boldsymbol{u} \cdot \boldsymbol{v} \geq-1, \forall \boldsymbol{u} \in \Delta\right\}
$$

is also a lattice polytope in $\mathbb{Z}^{n}$. For $n=2$, it is not hard to show that $\Delta$ is reflexive iff there is only one interior point. ${ }^{2}$ Hence, we can always choose this unique interior point as the origin.

However, in this paper, we will contemplate $2 \mathrm{~d}$ polytopes with two interior points. Hence, they are not reflexive, and we have two choices of origins. This would lead to a different discussion on the compact toric surface $X(\Delta)$ in section 7 . Here, we will first focus on the rational polyhedral cone generated by the vertices of the polytope/toric diagram $\Delta$ in $3 \mathrm{~d}^{3}$

The affine toric CY 3-fold. We take the origin $(0,0,0) \in \mathbb{Z}^{3}=: M$, and let the vertices in the polygon be $\boldsymbol{u}_{i}^{\prime}=\left(\boldsymbol{u}_{i}, 1\right) \in \mathbb{Z}^{3}$. Then these vectors generate a cone $\sigma$ with the origin

\footnotetext{
${ }^{2}$ We acknowledge Alexander Kasprzyk for pointing out that this statement (namely the "if" part, in other words, the " $\Leftarrow$ " direction) is not generally true when $n \neq 2$.

${ }^{3}$ Notice that this construction can be done in any dimension, but here we are just talking about lattice polygons.
} 
as the apex to the vertices of $\Delta$ :

$$
\sigma=\left\{\sum_{i} \lambda_{i} \boldsymbol{u}_{i}^{\prime}: \lambda_{i} \geq 0\right\} \subset M \otimes_{\mathbb{Z}} \mathbb{R}=: M_{\mathbb{R}} .
$$

The dual cone lives in the dual lattice $N_{\mathbb{R}}$ where $N:=\operatorname{Hom}(M, \mathbb{Z})$ :

$$
\sigma^{\vee}=\left\{\boldsymbol{w} \in N_{\mathbb{R}}: \boldsymbol{w} \cdot \boldsymbol{u} \geq 0, \forall \boldsymbol{u} \in \sigma\right\}
$$

Then we have the algebra $\mathbb{C}\left[\sigma^{\vee} \cap N\right]$ spanned over $\mathbb{C}$ by the points in $\sigma \cap M$. We can therefore define an affine toric variety $\mathcal{X}$ to be the maximal spectrum of this semigroup ring:

$$
\mathcal{X} \cong \operatorname{Spec}_{\max } \mathbb{C}\left[\sigma^{\vee} \cap N\right] .
$$

Since the endpoints of $\sigma$ live on the same (hyper)plane, $\mathcal{X}$ is a Gorenstein singularity, and hence can be resolved to a CY 3-fold, although being co-hyperplanar makes it noncompact $[13,46,47]$.

The Higgs-Kibble mechanism. The Higgs(-Kibble) mechanism [48-50] has a natural interpretation in the toric diagrams. Blowing down points of the polytopes corresponds to higgsing while blowing up points is unhiggsing. All the 45 toric diagrams (and corresponding quiver gauge theories) can be obtained by higgsing the same parent theory. This is analyzed in appendix C.

\section{$2.2 \quad$ Brane tilings}

As mentioned in section 1, the junction of $N$ D5-branes and one NS5-brane can be plotted on the torus. Given a toric diagram, we can draw the outer normal vector to each segment separated by the perimeter points of the polytope. Then we put these vectors on the torus, which will divide the torus into different regions. Each region is a bound state of 5 -branes, including $(N, 0)$ and $(N, \pm 1)$ 5-branes. Every time when we move from one region to another, we will cross a vector. If we cross the vector from left (right) to right (left), then the NS5 charge is increased (decreased) by 1 . For instance, the NS5 cycles of $\mathbb{C}^{3} / \mathbb{Z}_{5}$ $(1,2,2)$ which we will study later in section 3.2 is (figure taken from [51], figure 29):
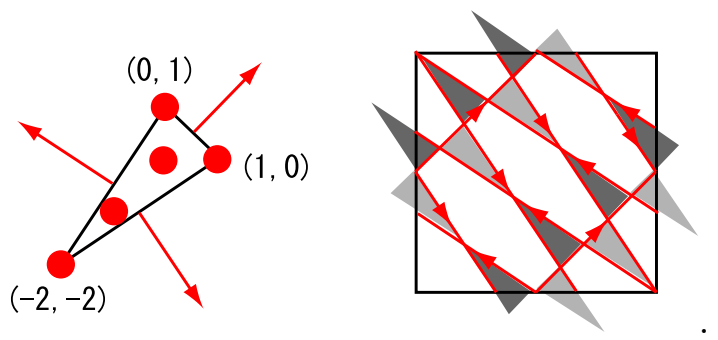

Then we can obtain a bipartite graph by taking the $(N, \pm 1)$ regions to be white/black nodes. The $(N, 0)$ regions give faces in the tiling. The intersection points of the branes, for which we have massless open strings, correspond to edges in the tiling. As the open 


\begin{tabular}{|c|c|c|}
\hline Fivebrane diagram & Brane Tiling & Quiver \\
\hline$(N, 1)$ brane & white node & superpotential term $(+)$ \\
\hline$(N,-1)$ brane & black node & superpotential term $(-)$ \\
\hline$(N, 0)$ brane & face & gauge node/group \\
\hline open string & edge & bifundamental \\
\hline
\end{tabular}

Table 2. The relation for fivebrane diagrams, brane tilings and quivers.

strings/bifundamentals are oriented, every loop surrounding the white/black node is clockwise/counterclockwise, which gives a sign in the corresponding superpotential term. For instance, the above example leads to the brane tiling in (3.11). Since the bipartite graph is periodic, the fundamental region is in a red box. From fivebrane diagrams/brane tilings, we can read off the quivers. This is summarized in table 2. Readers are referred to [51, 52] for a detailed discussion.

Quivers. In our context, our quivers only have two objects: round nodes and arrows. Each round node corresponds to a gauge group, which is always unitary here. Also, as we are contemplating toric quivers, viz, quivers in the toric phases, the ranks of nodes in one quiver are always the same. Each arrow connects two gauge nodes. These arrows correspond to the matter fields transform under fundamental and anti-fundamental representations under the two gauge groups. We can write a $G \times E$ matrix, where $G$ is the number of gauge nodes ${ }^{4}$ and $E$ is the number of edges/bifundamentals, called incidence matrix $d$ to encode the quiver data. If the arrow leaves the node $i$, viz, the bifundamental $X_{i j}$, then the corresponding entry is assigned 1 . Likewise, if the arrow comes into the node $i$, viz, the bifundamental $X_{j i}$, then the entry is -1 . Otherwise, the entry is 0 .

Perfect matchings and charges. It is always to possible to find a set $p_{\alpha}$ of bifundamentals that connect all the nodes in the brane tiling precisely once. This set $p_{\alpha}$ is known as a perfect matching. A new basis of fields in the language of gauged linear sigma model (GLSM) [53] can be naturally defined from the bifundamental fields [2]. The number of GLSM fields is the number of perfect matchings $c$. Then we can write the $P_{E \times c}$ perfect matching matrix $P$ which encodes the relation between the two sets of matter fields. For instance, the first row in (3.4) indicates that

$$
X_{12}^{1}=q_{1} s_{2} s_{4} s_{6} r_{5} p_{2} .
$$

As the F-terms come from $\partial W / \partial X_{i j}=0$, where $W$ is the superpotential and $X_{i j}$ 's are the bifundamentals, one can show that the charges of GLSM fields under the F-term constraints are given by the $F$-term charge matrix of size $(c-G-2) \times c$ :

$$
Q_{F}=\operatorname{ker}(P) .
$$

\footnotetext{
${ }^{4}$ As we will see shortly, the number of nodes $G$ is always equal to the number of unit simplices under full triangulation of the toric diagram. This in turn equals twice the area of the toric diagram where the area of a unit triangle is not normalized here, i.e., equals $1 / 2$.
} 
From [53], we know that the D-terms in terms of the bifundamentals $X_{a}$ 's are

$$
D_{i}=-e^{2}\left(\sum_{a} d_{i a}\left|X_{a}\right|^{2}-\zeta_{i}\right),
$$

where $e$ is the gauge coupling and $d$ is the incidence matrix. The $\zeta_{i}$ 's are Fayet-Iliopoulos (FI) parameters. In fact, as shown in [1,2], the FI parameters encode the resolutions of toric singularities. In the matrix form, this reads

$$
\delta \cdot\left|X_{a}\right|^{2}=\zeta
$$

where $\delta$ is the reduced quiver matrix ${ }^{5}$ of size $(G-1) \times E$. This can be related to perfect matching matrix via $[2,25]$

$$
\delta=Q_{D} P^{\mathrm{T}}
$$

where $Q_{D}$ is a $(G-1) \times c$ matrix. As $Q_{D}$ encodes the GLSM charges under D-term constraints, this is known as the D-term matrix.

In light of GLSM, the F- and D-terms can be treated on an equal footing. Hence, the two charge matrices can be concatenated to a $(c-3) \times c$ matrix, known as the total charge matrix [2]:

$$
Q_{t}=\left(\begin{array}{c}
Q_{F} \\
Q_{D}
\end{array}\right) .
$$

As the F-terms must vanish while the D-terms are adjusted by the FI parameters, the last column is always in the form $(\mathbf{0}, \boldsymbol{\zeta})^{\mathrm{T}}$. Hence, we will always omit the last column. Then taking the kernel yields

$$
G_{t}=\operatorname{ker}\left(Q_{t}\right) .
$$

This matrix $G_{t}$ exactly encodes the information of the toric diagrams. Each column is the coordinate of a vertex in the polytope (thus, the last row of $G_{t}$ is $(1, \ldots, 1)$ ). Therefore, every vertex is assigned to some GLSM field(s). Each corner (aka extremal) point always correspond to one GLSM field with non-zero R-charge. On the other hand, non-extremal points corresponds to multiple GLSM fields all with zero R-charges.

Toric/Seiberg duality. The toric/Seiberg duality $[4,54,55]$ is a duality among theories that have the same IR fixed point under RG flow. As we are always staying in the toric phases, there will be no fractional branes, and hence our theories keep superconformal and the quivers have nodes of the same rank as aforementioned. The dual quiver gauge theories all have the same moduli space/Higgs branch, which is exactly the toric CY cone corresponding to the toric diagram.

Therefore, we can use toric duality to obtain different quivers of the same toric diagram with the following steps:

\footnotetext{
${ }^{5}$ In $[1,2]$, the reduced quiver matrix was originally denoted by $\Delta$. However, as $\Delta$ represents polyotpes here, we use $\delta$ for the matrix to avoid any possible confusion.
} 
1. As Seiberg duality takes $\mathrm{SU}\left(N_{c}\right)$ gauge group with $N_{f}$ fundamentals and $N_{f}$ bifundamentals to $\mathrm{SU}\left(N_{f}-N_{c}\right)$ gauge group, in the toric phase, only nodes satisfying $N_{f}=2 N_{c}$ can be dualized. ${ }^{6}$ We first scale the gauge couplings of gauge groups other than the chosen node $i$ to zero, and the fields not connected to $i$ decouple. Then the bifundamentals connected to $i$ is reduced to (anti-)fundamentals under the flavour symmetry. Since duality requires the dual quarks to transform in the conjugate (flavour) representations to the original ones, the directions of the $2 N_{f}$ arrows should be reversed. The overall result is that every time we perform such duality, we flip one node $i$ in the quiver so that the arrows connecting to it are all reversed.

2. To be anomaly-free, new arrows needs to be added among pairs of nodes adjacent to dualized node $i$ so as to keep them balanced. This is just the quarks-to-meson map $Q_{i} \tilde{Q}^{j} \rightarrow M_{i}^{j}$. As the flavours groups are gauged back, these mesons are promoted to bifundamentals. Overall, we are adding $N_{f}$ arrows to the pairs of unbalanced nodes after we flip the dualized node.

In cluster algebra, the whole process is known as the quiver mutations [56]. For the superpotential, the composite singlets are replaced with the new mesons, and adding new cubic terms couples the mesons to magnetic flavours. This may make some fields massive, so we need to integrate them out as they become non-dynamical when flowing to IR. In terms of brane tilings, the technique called urban renewal can be applied to obtain dual tilings. For more details in Seiberg duality in quiver gauge theories, one is referred to, for example, $[9,57-59]$.

\subsection{The moduli spaces}

The master space $\mathcal{F}^{b}[60,61]$ is a combination of baryonic and mesonic moduli spaces defined as the symplectic quotient of the perfect matching ring: ${ }^{7}$

$$
\mathcal{F}^{b}=\mathbb{C}^{c}\left[p_{1}, \ldots, p_{c}\right] / / Q_{F} .
$$

The global symmetry. The master space has global symmetry that can be divided into two parts:

- The mesonic symmetry is $\mathrm{U}(1)^{3}$ or its enhancement with rank 3 . It may be enhanced to $\mathrm{SU}(2) \times \mathrm{U}(1)^{2}, \mathrm{SU}(2)^{2} \times \mathrm{U}(1)$ or $\mathrm{SU}(3) \times \mathrm{U}(1)$. The enhancement is determined by the duplicated columns in $Q_{t}$. In particular, there is always a $\mathrm{U}(1)$ which is the R-symmetry.

- The baryonic symmetry is $\mathrm{U}(1)^{G-1}$ or its enhancement with rank $(G-1)$. It consists of non-anomalous and anomalous symmetries. The non-anomalous symmetry is always $\mathrm{U}(1)^{N_{P}-3}$, where $N_{P}$ is the number of perimeter points in the polytope. The

\footnotetext{
${ }^{6}$ As we will take only U(1) nodes for simplicity, this means we can only choose nodes with two arrows in and two arrows out. However, we should remember that any node can be dualized if we do not restrict to staying in toric phases.

${ }^{7}$ Strictly speaking, this is the largest irreducible component, known as the coherent component, of the master space rather than $\mathcal{F}^{b}$ itself. Nevertheless, we will solely focus on the coherent component and make this abuse.
} 
anomalous symmetry is $\mathrm{U}(1)^{2 I}$ or an enhancement of rank $2 I$, where $I$ is the number of interior points. The enhancement is determined by the repeated columns in $Q_{F}$. The non-abelian enhancement of anomalous symmetry is also known as hidden symmetry.

Notice that the combination in the baryonic symmetry is actually the Pick's theorem:

$$
\frac{G}{2}=I+\frac{N_{P}}{2}-1=A,
$$

where $A$ is the (unnormalized) area of the toric diagram.

The mesonic moduli space and Hilbert series. The mesonic moduli space $\mathcal{M}$ is a subspace of $\mathcal{F}^{b}$ :

$$
\mathcal{M}=\mathcal{F}^{b} / / Q_{D}=\left(\mathbb{C}^{c}\left[p_{1}, \ldots, p_{c}\right] / / Q_{F}\right) / / Q_{D} .
$$

We can use the (mesonic) Hilbert series (aka Hilbert-Poincaré series) to desribe the moduli space. The Hilbert series is a generating function that enumerates the invariant monomials under the group action. Physically, it counts the gauge invariant operators of each degree in the chiral ring. As aforementioned, the moduli space coincides the toric CY 3 -fold $\mathcal{X}$. Hence, we can use the following formula to compute the Hilbert series. The (refined) Hilbert series for a toric CY $n$-fold cone can be computed as [62, 63]

$$
H S=\sum_{i=1}^{r} \prod_{j=1}^{n}\left(1-\boldsymbol{t}^{\boldsymbol{u}_{\boldsymbol{i}, \boldsymbol{j}}}\right)^{-1} .
$$

The number $r$ is the number of $(n-1)$-dimensional simplices under triangulation. The index $j$ runs over the $n$ faces of each simplex. The vector $\boldsymbol{u}_{\boldsymbol{i}, \boldsymbol{j}}$ is an $n$-vector inner normal to the $j^{\text {th }}$ face of the $i^{\text {th }}$ simplex, and $\boldsymbol{t}$ are the fugacities $t_{1}, \ldots, t_{n}$. Then $\boldsymbol{t}^{\boldsymbol{u}_{\boldsymbol{i}, \boldsymbol{j}}}=\prod_{k=1}^{n} t_{k}^{\boldsymbol{u}_{i, j}(k)}$, multiplied by the $k^{\text {th }}$ component of $\boldsymbol{u}$. One can also use Molien-Weyl integral to compute Hilbert series of the Higgs branch [64]. The two results should be the same under some fugacity map.

\subsection{Volume minimization}

As $\mathcal{X}$ of complex dimension $n$ is the Kähler cone over the Sasaki-Einstein manifold $Y=$ $\left.\mathcal{X}\right|_{r=1}$ of real dimension $(2 n-1)$ :

$$
\mathrm{d} s^{2}(\mathcal{X})=\mathrm{d} r^{2}+r^{2} \mathrm{~d} s^{2}(Y)
$$

the volume of $Y$ is then $[62,63]$

$$
\operatorname{vol}(Y)=2 n \int_{0}^{1} \mathrm{~d} r r^{2 n-1} \operatorname{vol}(Y)=2 n \operatorname{vol}\left(\left.\mathcal{X}\right|_{r \leq 1}\right)=2 n \int_{r \leq 1} \frac{\omega^{n}}{n !},
$$

where $\omega$ is the Kähler form of $\mathcal{X}$. We are now going to see that the volume of the SasakiEinstein base is closely related to the R-charges of the fields in our theory.

The Reeb vector $K:=\mathcal{J}(r \partial / \partial r)$ is the Killing vector of $Y$, where $\mathcal{J}$ is the complex structure of $\mathcal{X}$. Since the torus action $\mathbb{T}^{n}$ of the toric $\mathcal{X}$ leaves $\omega$ invariant, we can take 
the vector fields $\partial / \partial \phi_{i}$ to be the generators of the action with $\phi_{i} \sim \phi_{i}+2 \pi$. Then the reeb vector reads $K=b_{i} \partial / \partial \phi_{i}$, where the components $b_{i}$ 's are algebraic numbers, with the last component $b_{n}$ set to be $n$.

In $[62,63]$, the volume function of $Y$, which is shown to be related to the Reeb vector components, is introduced to be

$$
V\left(b_{i} ; Y\right)=\frac{\operatorname{vol}(Y)}{\operatorname{vol}\left(S^{2 n-1}\right)}
$$

such that the volume of the $(2 n-1)$-sphere,

$$
\operatorname{vol}\left(S^{2 n-1}\right)=\frac{2 \pi^{n}}{(n-1) !}
$$

is normalized. Then the volume function is related to the Hilbert series of $\mathcal{X}$ via $^{8}$

$$
V\left(b_{i} ; Y\right)=\lim _{\mu \rightarrow 0} \mu^{n} H S\left(t_{i}=\exp \left(-\mu b_{i}\right) ; \mathcal{X}\right)
$$

It is known that $V$ always admits precisely one positive minimum $V_{\min }$. Since the Reeb vector is algebraic, $V_{\min }$ is also an algebraic number.

For toric threefolds, in [65], it was shown that the $a$-function, in terms of the volume function, can be expressed as

$$
a(R)=\frac{1}{4 V}
$$

where $R$ denotes the R-charges of the superconformal theory. A procedure known as $a$ maximization can be used to determine the R-charges [66-68]. The central charges $a$ and $c$ of the SCFT in $4 \mathrm{~d}$ are

$$
a(R)=\frac{3}{32}\left(3 \operatorname{Tr} R^{3}-\operatorname{Tr} R\right), c=\frac{1}{32}\left(9 \operatorname{Tr} R^{3}-5 \operatorname{Tr} R\right),
$$

where $\operatorname{Tr} R^{3}$ and $\operatorname{Tr} R$ are 't Hooft anomalies. In general, as we have flavour symmetries in IR, a possible candidate is

$$
R_{t}=R_{0}+\sum_{i} t_{i} F_{i}
$$

where $F_{i}$ 's are the charges of global non-R symmetries and $R_{t}$ is called the trial $\mathrm{R}$-charge. According to [66], the U(1) R-symmetry should satisfy

$$
9 \operatorname{Tr}\left(R^{2} F_{i}\right)=\operatorname{Tr} F_{i}, \operatorname{Tr}\left(R F_{i} F_{j}\right)<0,
$$

which can be translated into the maximization of $a\left(R_{t}\right)$. When the trial $a$-function is maximized, only the R-charge $R_{0}$ will make contribution. Thus, we see that $V_{\min }$ plays a crucial role in determining the R-charges.

\footnotetext{
${ }^{8}$ If we are taking outer normal vectors to the faces of simplices when computing the Hilbert series, the Hilbert series would just change by the fugacity map $t_{i} \rightarrow 1 / t_{i}$. As a result, the volume function would only differ by a minus sign.
} 
In light of quiver diagrams, let $X_{I}$ be the R-charges of the bifundamentals. Then the vanishing $\beta$-function from the theory being conformal yields

$$
\sum_{I} X_{I}=2, \sum_{I}\left(1-X_{I}\right)=2
$$

where the first sum is taken in each superpotential term and the second sum is taken with respect to each gauge node. Let $N_{W}$ be the number of superpotential terms, then we have $\left(G+N_{W}\right)$ equations for $E$ parameters in all, which in general are not all independent though $G+N_{W}=E$ as the bipartite graph is embedded on a torus. With these conditions, the $a$-function can be written as ${ }^{9}$

$$
a=\frac{3}{32}\left(2 G+\sum_{I}\left(3\left(X_{I}-1\right)^{3}-\left(X_{I}-1\right)\right)\right) .
$$

Anomaly cancellation implies $a=c$, viz, $\operatorname{Tr} R=0[69,70] .{ }^{10}$ Thus, we have

$$
a=\frac{9}{32}\left(G+\sum_{I}\left(X_{I}-1\right)^{3}\right) .
$$

As we have seen, this is equivalent to minimizing $V$, together with (2.26), we can solve for the R-charges of the bifundamentals, and hence the R-charges of GLSM fields as well.

Example. Let us consider the abelian orbifold $\mathbb{C}^{n} / \mathbb{Z}_{n}$ with orbifold action $(1, \ldots, 1)$ as an example. The Hilbert series reads

$$
\begin{aligned}
H S= & \left(\left(1-t_{n}^{-s} \prod_{i=1}^{n-1} t_{i}^{s}\right) \prod_{j=1}^{n-1}\left(1-t_{j}^{s}\right)\right)^{-1} \\
& +\sum_{i=1}^{n-1}\left(\left(1-t_{i}^{-s}\right)\left(1-t_{i}^{s n} t_{n}^{s} \prod_{j=1}^{n-1} t_{j}^{s}\right) \prod_{\substack{k=1 \\
k \neq i}}^{n-1}\left(1-t_{k}^{s} t_{i}^{-s}\right)\right)^{-1}
\end{aligned}
$$

where $s=(-1)^{n}$. As the limit picks out the leading order of $\mu$, the volume function is

$$
V=\frac{(-1)^{n} n^{n-1}}{\prod_{j=1}^{n-1}\left(\sum_{i=1}^{n-1} b_{i}-n b_{j}-b_{n}\right)} .
$$

Then taking $b_{n}=n$, we find that $V_{\min }=1 / n$ at $b_{1}=\cdots=b_{n-1}=0$. In quiver gauge theories, we have a unique toric quiver for each $n$. The R-charges of all the bifundamentals are $2 / n$. Hence, the R-charges of the $n$ GLSM fields corresponding to extremal points are all $2 / n$, with others vanishing. Interestingly, the Sasaki-Einstein base of $\mathbb{C}^{n}$ (whose toric diagram is the unit simplex) is the $(2 n-1)$-sphere. Hence, the volume function equals 1. As we will see in section 7.2 , it is not a coincidence to have $1 / n=V\left(S^{2 n-1}\right) /\left|\mathbb{Z}_{n}\right|$ here.

\footnotetext{
${ }^{9}$ Notice that this expression itself, which is generally true when we assume all the gauge groups have the same rank $N$ and normalize by $N^{2}$, does not require that the dimer is embedded on $\mathbb{T}^{2}$.

${ }^{10}$ The relevant anomalies are the ones of the R-symmetry current with itself or with the stress tensor, namely $\langle R R R\rangle$ or $\langle R T T\rangle$.
} 


\section{Five triangles}

Having warmed up with an explicit example of an orbifold, and illustrating it with all the relevant concepts, let us now proceed to study the polygons of our concern. As aforementioned, there are 45 lattice polygons investigated here, which are collected in appendix A (one can explicitly see the 2 interior points, one of which could be taken as the origin). We begin with the five triangles.

\subsection{Polytope 1: $\mathbb{C}^{3} / \mathbb{Z}_{6}(1,1,4)$}

The polytope is

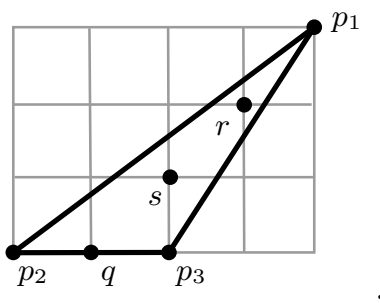

The brane tiling and the corresponding quiver are ${ }^{11}$
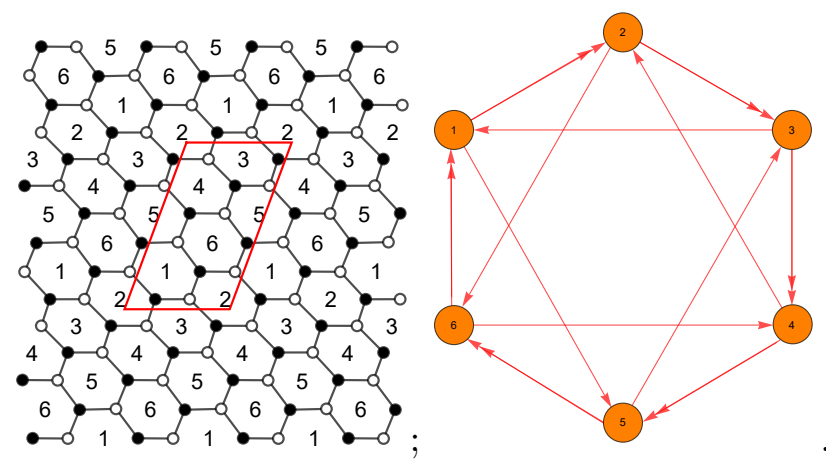

The superpotential is ${ }^{12}$

$$
\begin{aligned}
W= & X_{23}^{1} X_{34}^{2} X_{42}+X_{45}^{1} X_{56}^{2} X_{64}+X_{61}^{1} X_{12}^{2} X_{26}+X_{56}^{1} X_{61}^{2} X_{15}+X_{12}^{1} X_{23}^{2} X_{31}+X_{34}^{1} X_{45}^{2} X_{53} \\
& -X_{23}^{2} X_{34}^{1} X_{42}-X_{45}^{2} X_{56}^{1} X_{64}-X_{61}^{2} X_{12}^{1} X_{26}-X_{56}^{2} X_{61}^{1} X_{15}-X_{12}^{2} X_{23}^{1} X_{31} \\
& -X_{34}^{2} X_{45}^{1} X_{53} .
\end{aligned}
$$

\footnotetext{
${ }^{11}$ Notice that the numbers in the nodes are labels, not ranks.

${ }^{12}$ There is always a trace on the right hand side. For brevity, we will just omit it here. Alternatively, we can also think of it as the repeated lower indices being traced. Moreover, the upper indices are the labels of multiple bifundamentals between two nodes (rather than powers).
} 
The perfect matching matrix is

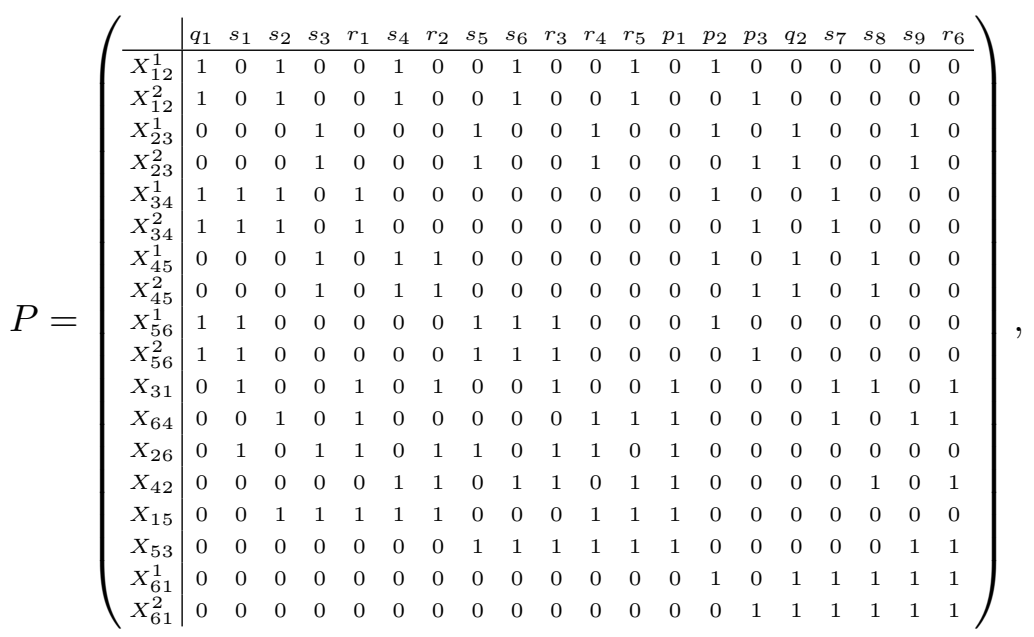

where the relations between bifundamentals and GLSM fields can be directly read off. Then we can get the total charge matrix:

$$
Q_{t}=\left(\begin{array}{cccccccccccccccccccc}
q_{1} & s_{1} & s_{2} & s_{3} & r_{1} & s_{4} & r_{2} & s_{5} & s_{6} & r_{3} & r_{4} & r_{5} & p_{1} & p_{2} & p_{3} & q_{2} & s_{7} & s_{8} & s_{9} & r_{6} \\
\hline 3 & -1 & -1 & 2 & 0 & -1 & 0 & -1 & 0 & 0 & 0 & 0 & 0 & -1 & -1 & 0 & 0 & 0 & 0 & 1 \\
2 & 0 & -1 & 1 & 0 & 0 & 0 & -1 & 0 & 0 & 0 & 0 & 0 & -1 & -1 & 0 & 0 & 0 & 1 & 0 \\
2 & -1 & 0 & 1 & 0 & -1 & 0 & 0 & 0 & 0 & 0 & 0 & 0 & -1 & -1 & 0 & 0 & 1 & 0 & 0 \\
2 & -1 & -1 & 1 & 0 & 0 & 0 & 0 & 0 & 0 & 0 & 0 & 0 & -1 & -1 & 0 & 1 & 0 & 0 & 0 \\
1 & 0 & 0 & 0 & 0 & 0 & 0 & 0 & 0 & 0 & 0 & 0 & 0 & -1 & -1 & 1 & 0 & 0 & 0 & 0 \\
2 & -1 & -1 & 1 & 0 & -1 & 0 & -1 & 0 & 0 & 0 & 0 & 1 & 0 & 0 & 0 & 0 & 0 & 0 & 0 \\
1 & 0 & -1 & 1 & 0 & -1 & 0 & -1 & 0 & 0 & 0 & 1 & 0 & 0 & 0 & 0 & 0 & 0 & 0 & 0 \\
1 & 0 & -1 & 0 & 0 & 0 & 0 & -1 & 0 & 0 & 1 & 0 & 0 & 0 & 0 & 0 & 0 & 0 & 0 & 0 \\
1 & -1 & 0 & 1 & 0 & -1 & 0 & -1 & 0 & 1 & 0 & 0 & 0 & 0 & 0 & 0 & 0 & 0 & 0 & 0 \\
0 & 0 & 0 & 1 & 0 & -1 & 0 & -1 & 1 & 0 & 0 & 0 & 0 & 0 & 0 & 0 & 0 & 0 & 0 & 0 \\
1 & -1 & 0 & 0 & 0 & -1 & 1 & 0 & 0 & 0 & 0 & 0 & 0 & 0 & 0 & 0 & 0 & 0 & 0 & 0 \\
1 & -1 & -1 & 0 & 1 & 0 & 0 & 0 & 0 & 0 & 0 & 0 & 0 & 0 & 0 & 0 & 0 & 0 & 0 & 0 \\
\hline 4 & -1 & -1 & 2 & 0 & 0 & 0 & 0 & 0 & 0 & 0 & 0 & 0 & -2 & -2 & 0 & 0 & 0 & 0 & 0 \\
2 & -1 & 0 & 2 & 0 & 0 & 0 & -1 & 0 & 0 & 0 & 0 & 0 & -1 & -1 & 0 & 0 & 0 & 0 & 0 \\
2 & -1 & 1 & 1 & 0 & -1 & 0 & 0 & 0 & 0 & 0 & 0 & 0 & -1 & -1 & 0 & 0 & 0 & 0 & 0 \\
2 & -2 & 0 & 1 & 0 & 0 & 0 & 1 & 0 & 0 & 0 & 0 & 0 & -1 & -1 & 0 & 0 & 0 & 0 & 0 \\
2 & -1 & 0 & 0 & 0 & 1 & 0 & 0 & 0 & 0 & 0 & 0 & 0 & -1 & -1 & 0 & 0 & 0 & 0 & 0
\end{array}\right)
$$

with kernel

$$
G_{t}=\left(\begin{array}{cccccccccccccccccccc}
q_{1} & s_{1} & s_{2} & s_{3} & r_{1} & s_{4} & r_{2} & s_{5} & s_{6} & r_{3} & r_{4} & r_{5} & p_{1} & p_{2} & p_{3} & q_{2} & s_{7} & s_{8} & s_{9} & r_{6} \\
\hline-1 & 0 & 0 & 0 & 1 & 0 & 1 & 0 & 0 & 1 & 1 & 1 & 2 & -2 & 0 & -1 & 0 & 0 & 0 & 1 \\
2 & 1 & 1 & 1 & 0 & 1 & 0 & 1 & 1 & 0 & 0 & 0 & -1 & 4 & 0 & 2 & 1 & 1 & 1 & 0 \\
1 & 1 & 1 & 1 & 1 & 1 & 1 & 1 & 1 & 1 & 1 & 1 & 1 & 1 & 1 & 1 & 1 & 1 & 1 & 1
\end{array}\right)
$$

From $G_{t}$, we can get the GLSM fields associated to each point as shown in (3.1), where

$$
q=\left\{q_{1}, q_{2}\right\}, r=\left\{r_{1}, \ldots, r_{6}\right\}, s=\left\{s_{1}, \ldots, s_{9}\right\} .
$$

From $Q_{t}$ (and $Q_{F}$ ), the mesonic symmetry reads $\mathrm{SU}(2) \times \mathrm{U}(1) \times \mathrm{U}(1)_{\mathrm{R}}$ and the baryonic symmetry reads $\mathrm{U}(1)_{\mathrm{h}}^{4} \times \mathrm{U}(1)$, where the subscripts "R" and "h" indicate R- and hidden symmetries respectively. 
The Hilbert series of the toric cone is

$$
\begin{aligned}
H S= & \frac{1}{\left(1-\frac{t_{2}}{t_{1}}\right)\left(1-\frac{t_{2}^{2}}{t_{1}}\right)\left(1-\frac{t_{1}^{2} t_{3}}{t_{2}^{3}}\right)}+\frac{1}{\left(1-t_{1}\right)\left(1-\frac{t_{1}}{t_{2}}\right)\left(1-\frac{t_{2} t_{3}}{t_{1}^{2}}\right)} \\
& +\frac{1}{\left(1-\frac{t_{1}}{t_{2}}\right)\left(1-\frac{t_{1}^{2}}{t_{2} t_{3}}\right)\left(1-\frac{t_{2}^{2} t_{3}^{2}}{t_{1}^{3}}\right)}+\frac{1}{\left(1-\frac{t_{1}^{3}}{t_{2}^{4}}\right)\left(1-\frac{t_{2}}{t_{1}}\right)\left(1-\frac{t_{2}^{3}}{t_{1}^{2} t_{3}}\right)} \\
& +\frac{1}{\left(1-\frac{t_{1}}{t_{2}^{2}}\right)\left(1-\frac{t_{2}}{t_{1}}\right)\left(1-t_{2} t_{3}\right)}+\frac{1}{\left(1-\frac{1}{t_{1}}\right)\left(1-\frac{t_{1}}{t_{2}}\right)\left(1-t_{2} t_{3}\right)} .
\end{aligned}
$$

The volume function is then

$$
V=-\frac{18}{\left(b_{2}+3\right)\left(-3 b_{1}+2 b_{2}+6\right)\left(-3 b_{1}+4 b_{2}-6\right)} .
$$

Minimizing $V$ yields $V_{\min }=1 / 6$ at $b_{1}=b_{2}=0$. Thus, $a_{\max }=3 / 2$. Together with the superconformal conditions, we can solve for the R-charges of the bifundamentals, which are $X_{I}=2 / 3$ for any $I$, viz, for all the bifundamentals. Hence, the R-charges of GLSM fields are $p_{i}=2 / 3$ with others vanishing. ${ }^{13}$

\subsection{Polytope 2: $\mathbb{C}^{3} / \mathbb{Z}_{5}(1,2,2)$}

The polytope is

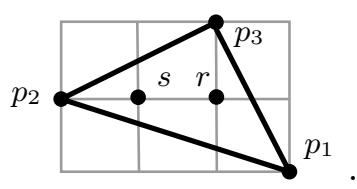

The brane tiling and the corresponding quiver are
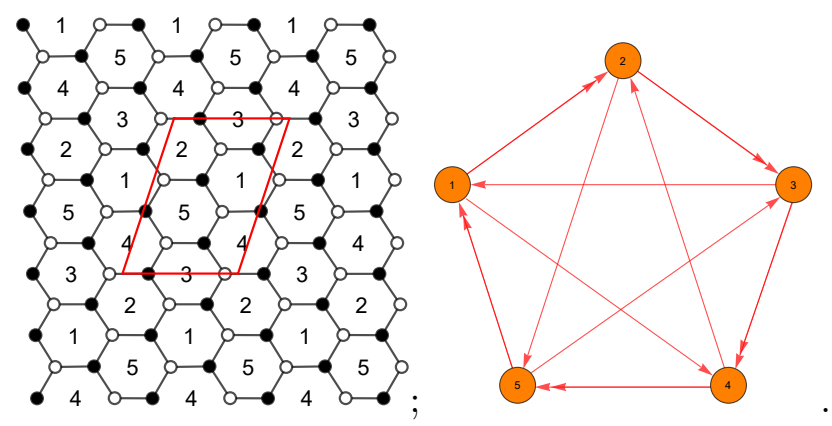

The superpotential is

$$
\begin{aligned}
W= & X_{12}^{1} X_{25} X_{51}^{2}+X_{12}^{2} X_{23}^{1} X_{31}+X_{23}^{2} X_{34}^{1} X_{42}+X_{34}^{2} X_{45}^{1} X_{53}+X_{45}^{2} X_{51}^{1} X_{14} \\
& -X_{12}^{2} X_{25} X_{51}^{1}-X_{12}^{1} X_{23}^{2} X_{31}-X_{23}^{1} X_{34}^{2} X_{42}-X_{34}^{1} X_{45}^{2} X_{53}-X_{45}^{1} X_{51}^{2} X_{14} .
\end{aligned}
$$

\footnotetext{
${ }^{13}$ We will simply use $p_{i}$ to denote the R-charge of $p_{i}$. This should not cause any confusion based on the context.
} 
The perfect matching matrix is

$$
P=\left(\begin{array}{c|ccccccccccccc} 
& p_{1} & p_{2} & s_{1} & s_{2} & s_{3} & r_{1} & s_{4} & r_{2} & s_{5} & r_{3} & r_{4} & r_{5} & p_{3} \\
\hline X_{12}^{1} & 1 & 0 & 0 & 0 & 1 & 0 & 1 & 0 & 0 & 0 & 0 & 1 & 0 \\
X_{12}^{2} & 0 & 1 & 0 & 0 & 1 & 0 & 1 & 0 & 0 & 0 & 0 & 1 & 0 \\
X_{23}^{1} & 1 & 0 & 0 & 1 & 0 & 0 & 0 & 0 & 1 & 0 & 1 & 0 & 0 \\
X_{23}^{2} & 0 & 1 & 0 & 1 & 0 & 0 & 0 & 0 & 1 & 0 & 1 & 0 & 0 \\
X_{34}^{1} & 1 & 0 & 1 & 0 & 0 & 0 & 1 & 1 & 0 & 0 & 0 & 0 & 0 \\
X_{34}^{2} & 0 & 1 & 1 & 0 & 0 & 0 & 1 & 1 & 0 & 0 & 0 & 0 & 0 \\
X_{45}^{1} & 1 & 0 & 0 & 1 & 1 & 1 & 0 & 0 & 0 & 0 & 0 & 0 & 0 \\
X_{45}^{2} & 0 & 1 & 0 & 1 & 1 & 1 & 0 & 0 & 0 & 0 & 0 & 0 & 0 \\
X_{51}^{1} & 1 & 0 & 1 & 0 & 0 & 0 & 0 & 0 & 1 & 1 & 0 & 0 & 0 \\
X_{51}^{2} & 0 & 1 & 1 & 0 & 0 & 0 & 0 & 0 & 1 & 1 & 0 & 0 & 0 \\
X_{31} & 0 & 0 & 1 & 0 & 0 & 1 & 0 & 1 & 0 & 1 & 0 & 0 & 1 \\
X_{25} & 0 & 0 & 0 & 1 & 0 & 1 & 0 & 1 & 0 & 0 & 1 & 0 & 1 \\
X_{42} & 0 & 0 & 0 & 0 & 1 & 1 & 0 & 0 & 0 & 1 & 0 & 1 & 1 \\
X_{14} & 0 & 0 & 0 & 0 & 0 & 0 & 1 & 1 & 0 & 0 & 1 & 1 & 1 \\
X_{53} & 0 & 0 & 0 & 0 & 0 & 0 & 0 & 0 & 1 & 1 & 1 & 1 & 1
\end{array}\right)
$$

where the relations between bifundamentals and GLSM fields can be directly read off. Then we can get the total charge matrix:

$$
Q_{t}=\left(\begin{array}{ccccccccccccc}
p_{1} & p_{2} & s_{1} & s_{2} & s_{3} & r_{1} & s_{4} & r_{2} & s_{5} & r_{3} & r_{4} & r_{5} & p_{3} \\
\hline 2 & 2 & -1 & -1 & -1 & 0 & -1 & 0 & -1 & 0 & 0 & 0 & 1 \\
1 & 1 & 0 & 0 & -1 & 0 & -1 & 0 & -1 & 0 & 0 & 1 & 0 \\
1 & 1 & 0 & -1 & 0 & 0 & -1 & 0 & -1 & 0 & 1 & 0 & 0 \\
1 & 1 & -1 & 0 & -1 & 0 & 0 & 0 & -1 & 1 & 0 & 0 & 0 \\
1 & 1 & -1 & -1 & 0 & 0 & -1 & 1 & 0 & 0 & 0 & 0 & 0 \\
1 & 1 & -1 & -1 & -1 & 1 & 0 & 0 & 0 & 0 & 0 & 0 & 0 \\
\hline 0 & 0 & -1 & 1 & 0 & 0 & 1 & 0 & -1 & 0 & 0 & 0 & 0 \\
0 & 0 & -1 & 0 & -1 & 0 & 2 & 0 & 0 & 0 & 0 & 0 & 0 \\
0 & 0 & -2 & 0 & 0 & 0 & 1 & 0 & 1 & 0 & 0 & 0 & 0 \\
0 & 0 & -1 & -1 & 1 & 0 & 1 & 0 & 0 & 0 & 0 & 0 & 0
\end{array}\right)
$$

with kernel

$$
G_{t}=\left(\begin{array}{ccccccccccccc}
p_{1} & p_{2} & s_{1} & s_{2} & s_{3} & r_{1} & s_{4} & r_{2} & s_{5} & r_{3} & r_{4} & r_{5} & p_{3} \\
\hline-3 & 0 & -1 & -1 & -1 & 0 & -1 & 0 & -1 & 0 & 0 & 0 & 1 \\
5 & 0 & 2 & 2 & 2 & 1 & 2 & 1 & 2 & 1 & 1 & 1 & 0 \\
1 & 1 & 1 & 1 & 1 & 1 & 1 & 1 & 1 & 1 & 1 & 1 & 1
\end{array}\right) .
$$

From $G_{t}$, we can get the GLSM fields associated to each point as shown in (3.10), where

$$
r=\left\{r_{1}, \ldots, r_{5}\right\}, s=\left\{s_{1}, \ldots, s_{5}\right\} .
$$

From $Q_{t}$ (and $Q_{F}$ ), the mesonic symmetry reads $\mathrm{SU}(2) \times \mathrm{U}(1) \times \mathrm{U}(1)_{\mathrm{R}}$ and the baryonic symmetry reads $\mathrm{U}(1)_{\mathrm{h}}^{4}$, where the subscripts " $\mathrm{R}$ " and " $\mathrm{h}$ " indicate $\mathrm{R}$ - and hidden symmetries respectively.

The Hilbert series of the toric cone is

$$
\begin{aligned}
H S= & \frac{1}{\left(1-\frac{t_{1}}{t_{3}}\right)\left(1-\frac{t_{1} t_{2}}{t_{3}}\right)\left(1-\frac{t_{3}^{3}}{t_{1}^{2} t_{2}}\right)}+\frac{1}{\left(1-t_{2}\right)\left(1-\frac{t_{2}}{t_{1}}\right)\left(1-\frac{t_{1} t_{3}}{t_{2}^{2}}\right)} \\
& +\frac{1}{\left(1-\frac{1}{t_{2}}\right)\left(1-t_{1} t_{2}^{2}\right)\left(1-\frac{t_{3}}{t_{1} t_{2}}\right)}+\frac{1}{\left(1-\frac{1}{t_{2}}\right)\left(1-\frac{1}{t_{1} t_{2}^{2}}\right)\left(1-t_{1} t_{2}^{3} t_{3}\right)} \\
& +\frac{1}{\left(1-t_{2}\right)\left(1-\frac{t_{1}}{t_{2}}\right)\left(1-\frac{t_{3}}{t_{1}}\right)} .
\end{aligned}
$$

The volume function is then

$$
V=-\frac{25}{\left(b_{1}-2 b_{2}+3\right)\left(2 b_{1}+b_{2}-9\right)\left(b_{1}+3 b_{2}+3\right)} .
$$


Minimizing $V$ yields $V_{\min }=1 / 5$ at $b_{1}=2, b_{2}=0$. Thus, $a_{\max }=5 / 4$. Together with the superconformal conditions, we can solve for the R-charges of the bifundamentals, which are $X_{I}=2 / 3$ for any $I$, viz, for all the bifundamentals. Hence, the R-charges of GLSM fields are $p_{i}=2 / 3$ with others vanishing. Such result is expected as the theory is in the same family of McKay quivers as the one in section 3.1.

\subsection{Polytope 3: $\mathbb{C}^{3} / \mathbb{Z}_{8}(1,3,4)$}

The polytope is

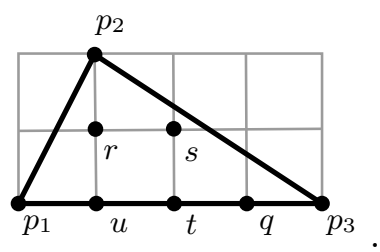

The brane tiling and the corresponding quiver are
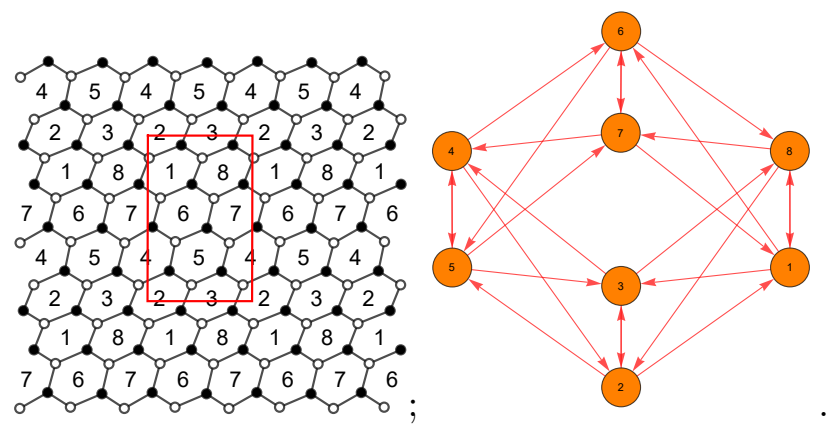

The superpotential is

$$
\begin{aligned}
W= & X_{82} X_{21} X_{18}+X_{13} X_{38} X_{81}+X_{34} X_{42} X_{23}+X_{25} X_{53} X_{32}+X_{46} X_{65} X_{54}+X_{57} X_{74} X_{45} \\
& +X_{68} X_{87} X_{76}+X_{67} X_{71} X_{16}-X_{23} X_{38} X_{82}-X_{13} X_{32} X_{21}-X_{25} X_{54} X_{42}-X_{34} X_{45} X_{53} \\
& -X_{57} X_{76} X_{65}-X_{46} X_{67} X_{74}-X_{16} X_{68} X_{81}-X_{18} X_{87} X_{71}
\end{aligned}
$$


The perfect matching matrix is

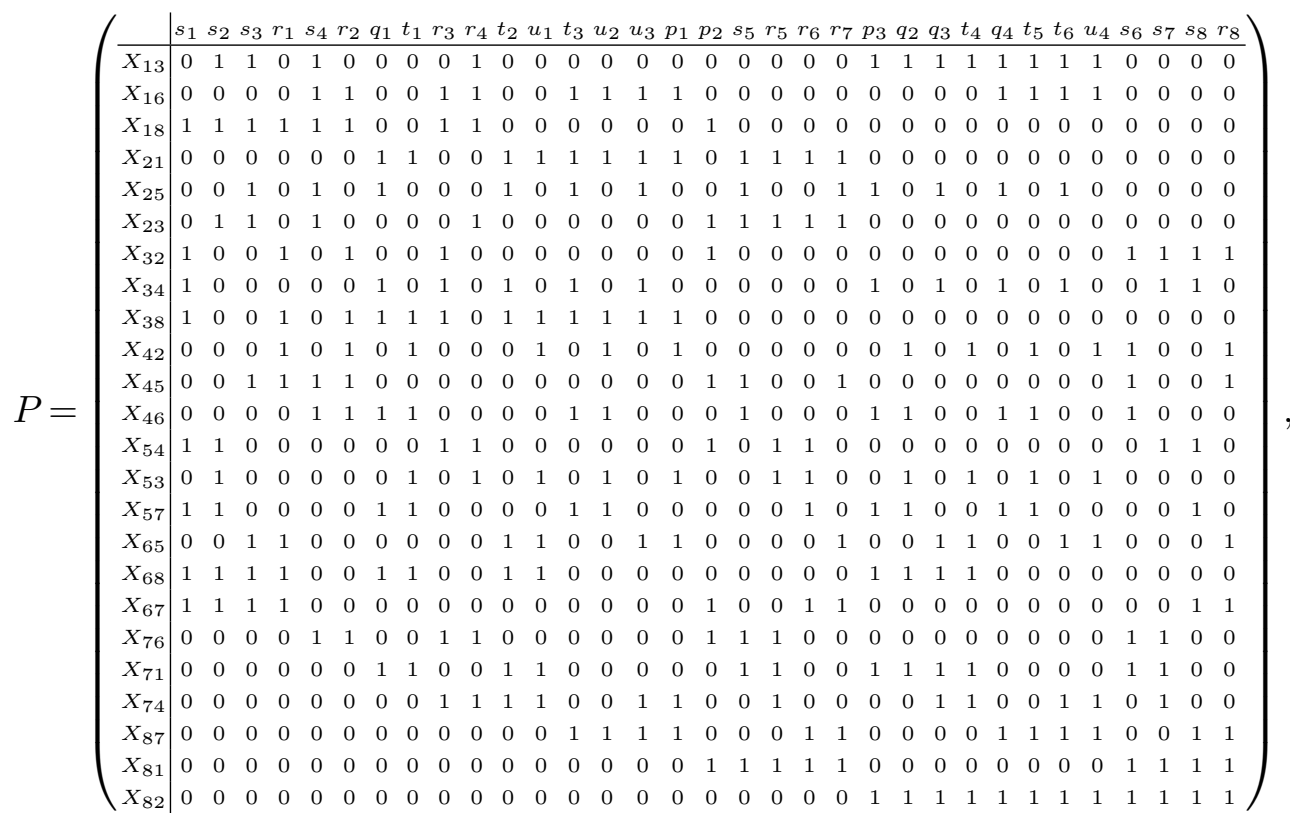

where the relations between bifundamentals and GLSM fields can be directly read off. Then we can get the total charge matrix:

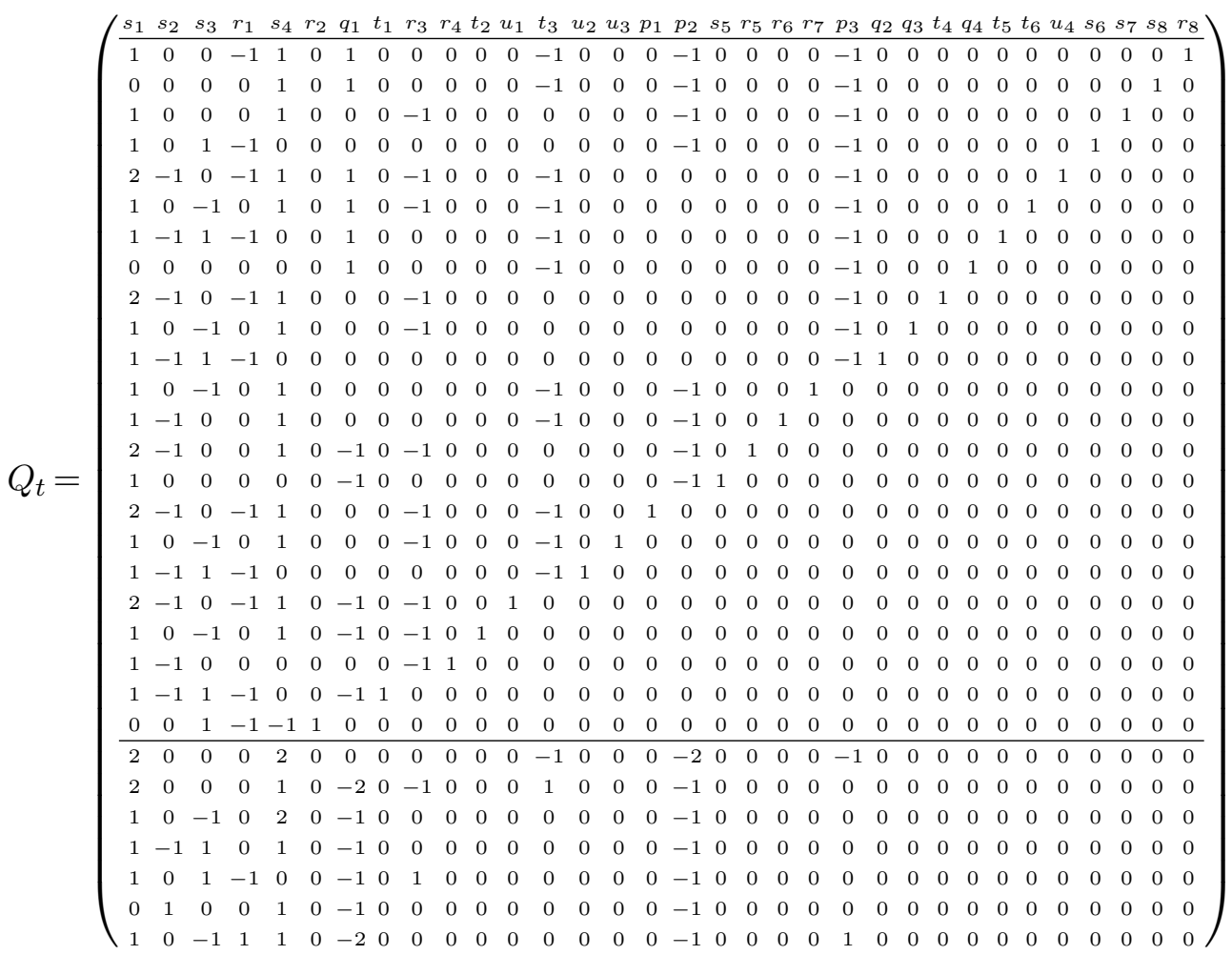

with kernel

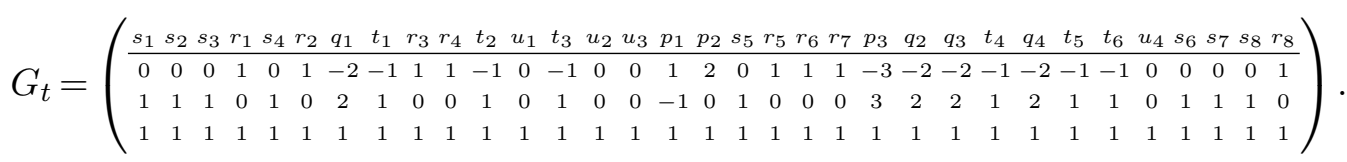


From $G_{t}$, we can get the GLSM fields associated to each point as shown in (3.19), where

$$
\begin{array}{ll}
q=\left\{q_{1}, \ldots, q_{4}\right\}, & r=\left\{r_{1}, \ldots, r_{8}\right\}, \quad s=\left\{s_{1}, \ldots, s_{8}\right\}, \\
t=\left\{t_{1}, \ldots, t_{6}\right\}, & u=\left\{u_{1}, \ldots, u_{4}\right\} .
\end{array}
$$

From $Q_{t}$ (and $Q_{F}$ ), the mesonic symmetry reads $\mathrm{U}(1)^{2} \times \mathrm{U}(1)_{\mathrm{R}}$ and the baryonic symmetry reads $\mathrm{U}(1)_{\mathrm{h}}^{4} \times \mathrm{U}(1)^{3}$, where the subscripts " $\mathrm{R}$ " and " $\mathrm{h}$ " indicate $\mathrm{R}$ - and hidden symmetries respectively.

The Hilbert series of the toric cone is

$$
\begin{aligned}
H S= & \frac{1}{\left(1-\frac{t_{1} t_{2}}{t_{3}}\right)\left(1-\frac{t_{1} t_{2}^{2}}{t_{3}}\right)\left(1-\frac{t_{3}^{3}}{t_{1} t_{2}{ }^{3}}\right)}+\frac{1}{\left(1-\frac{1}{t_{1}}\right)\left(1-\frac{t_{2}}{t_{1}}\right)\left(1-\frac{t_{1} t_{3}}{t_{2}}\right)} \\
& +\frac{1}{\left(1-t_{2} t_{3}\right)\left(1-\frac{t_{1} t_{2}}{t_{3}}\right)\left(1-\frac{t_{3}}{t_{1} t_{2}{ }^{2}}\right)}+\frac{1}{\left(1-\frac{1}{t_{1}}\right)\left(1-\frac{t_{1}}{t_{2}}\right)\left(1-t_{2} t_{3}\right)} \\
& +\frac{1}{\left(1-\frac{t_{3}}{t_{1}}\right)\left(1-t_{2} t_{3}\right)\left(1-\frac{t_{1}}{t_{2} t_{3}}\right)}+\frac{1}{\left(1-t_{1}\right)\left(1-\frac{1}{t_{2}}\right)\left(1-\frac{t_{2} t_{3}}{t_{1}}\right)} \\
& +\frac{1}{\left(1-t_{1}\right)\left(1-t_{2}\right)\left(1-\frac{t_{3}}{t_{1} t_{2}}\right)}+\frac{1}{\left(1-\frac{t_{1}}{t_{3}}\right)\left(1-t_{2} t_{3}\right)\left(1-\frac{t_{3}}{t_{1} t_{2}}\right)} .
\end{aligned}
$$

The volume function is then

$$
V=\frac{16}{\left(b_{2}+3\right)\left(-2 b_{1}+b_{2}-3\right)\left(2 b_{1}+3 b_{2}-9\right)} .
$$

Minimizing $V$ yields $V_{\min }=1 / 8$ at $b_{1}=2, b_{2}=-1$. Thus, $a_{\max }=2$. Together with the superconformal conditions, we can solve for the R-charges of the bifundamentals, which are $X_{I}=2 / 3$ for any $I$, viz, for all the bifundamentals. Hence, the R-charges of GLSM fields are $p_{i}=2 / 3$ with others vanishing.

\subsection{Polytope 4: $\mathbb{C}^{3} /\left(\mathbb{Z}_{2} \times \mathbb{Z}_{5}\right)(1,0,1)(0,1,4)$}

The polytope is

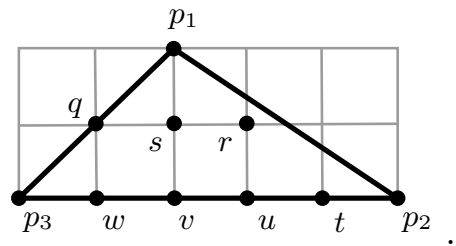

The brane tiling and the corresponding quiver are
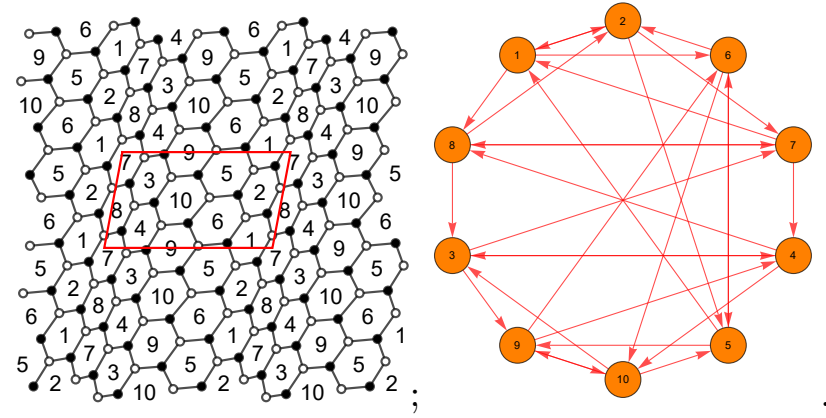
The superpotential is

$$
\begin{aligned}
W= & X_{16} X_{62} X_{21}+X_{25} X_{51} X_{12}+X_{59} X_{96} X_{65}+X_{56} X_{6,10} X_{10,5}+X_{4,10} X_{10,9} X_{94} \\
& +X_{39} X_{9,10} X_{10,3}+X_{37} X_{74} X_{43}+X_{34} X_{48} X_{83}+X_{18} X_{87} X_{71}+X_{27} X_{78} X_{82} \\
& -X_{16} X_{65} X_{51}-X_{25} X_{56} X_{62}-X_{6,10} X_{10,9} X_{96}-X_{59} X_{9,10} X_{10,5}-X_{39} X_{94} X_{43} \\
& -X_{34} X_{4,10} X_{10,3}-X_{48} X_{87} X_{74}-X_{37} X_{78} X_{83}-X_{18} X_{82} X_{21}-X_{12} X_{27} X_{71} .
\end{aligned}
$$


The perfect matching matrix is

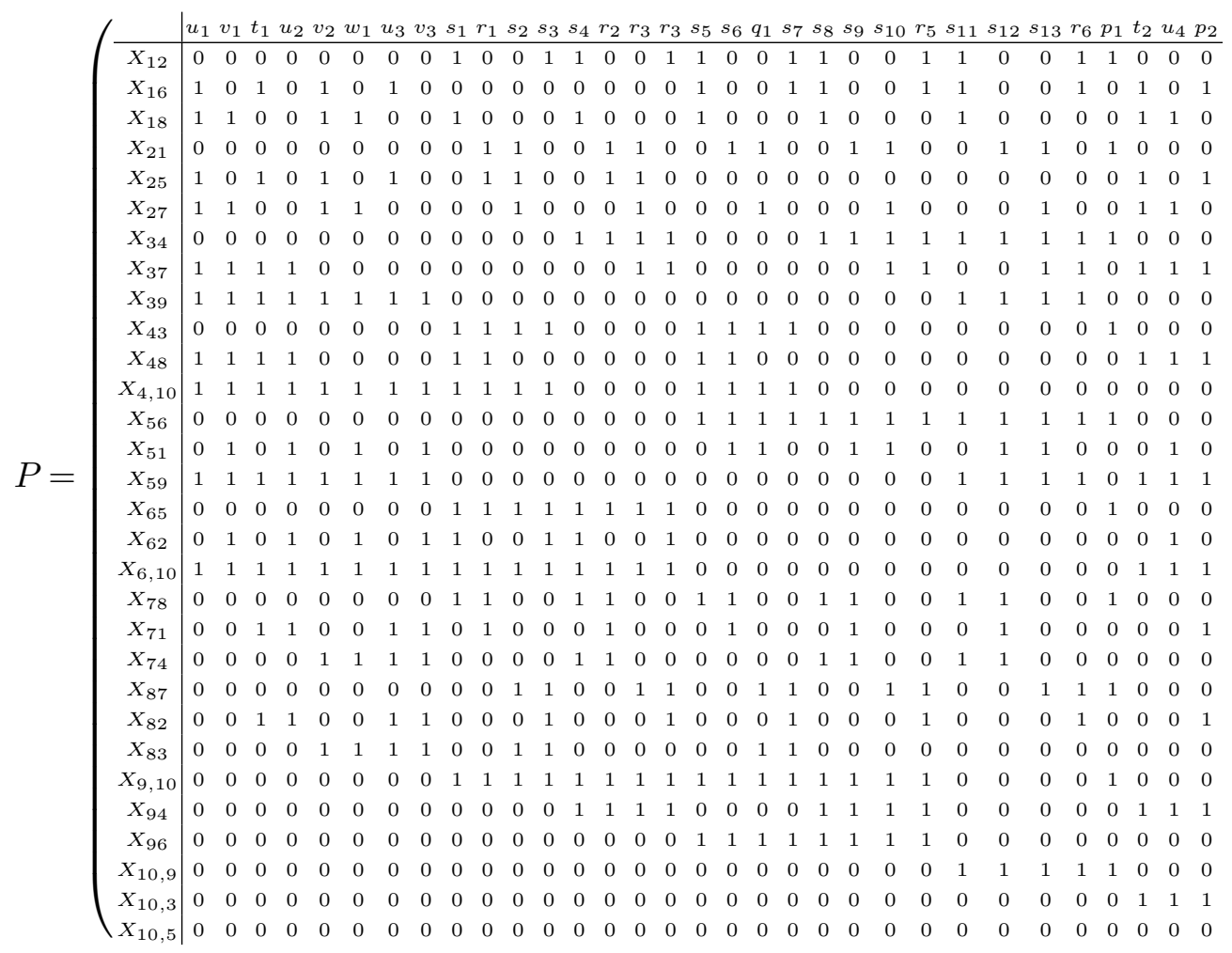

\begin{tabular}{|c|c|c|c|c|c|c|c|c|c|c|c|c|c|c|c|c|c|c|c|c|c|c|c|c|c|c|c|c|c|c|c|}
\hline 0 & 0 & 0 & 0 & 0 & 1 & 0 & 0 & 1 & 0 & 0 & 0 & 0 & 0 & 0 & 0 & 0 & 1 & 0 & 0 & 1 & 0 & 0 & $\begin{array}{lll}0 & 0\end{array}$ & $\begin{array}{ll}0 & 0\end{array}$ & 0 & 0 & 0 & 1 & 0 & 0 & 1 \\
\hline 0 & 1 & 0 & 1 & 0 & 1 & 0 & 0 & 1 & 1 & 0 & 1 & 0 & 1 & 0 & 1 & 0 & 0 & 0 & 0 & 0 & 1 & 0 & 10 & $\begin{array}{ll}0 & 1\end{array}$ & 0 & 1 & 0 & 0 & 0 & 0 & 0 \\
\hline 0 & 1 & 1 & 0 & 0 & 1 & 0 & 0 & 0 & 1 & 1 & 0 & 0 & 1 & 1 & 0 & 0 & 1 & 0 & 0 & 0 & 1 & 1 & $\begin{array}{ll}0 & 0\end{array}$ & $\begin{array}{ll}0 & 1\end{array}$ & 1 & 0 & 0 & 1 & 0 & 0 & 0 \\
\hline 0 & 0 & 0 & 0 & 0 & 0 & 1 & 1 & 0 & 0 & 0 & 0 & 0 & 0 & 0 & 0 & 0 & 0 & 1 & 1 & 0 & 0 & 0 & $\begin{array}{lll}0 & 0\end{array}$ & $\begin{array}{ll}0 & 0\end{array}$ & 0 & 0 & 0 & 0 & 1 & 1 & 0 \\
\hline 0 & 1 & 0 & 1 & 0 & 0 & 0 & 0 & 0 & 1 & 0 & 1 & 0 & 1 & 0 & 1 & 0 & 0 & 1 & 1 & 0 & 1 & 0 & 10 & $\begin{array}{ll}0 & 1\end{array}$ & 0 & 1 & 0 & 0 & 1 & 1 & 0 \\
\hline 0 & 1 & 1 & 0 & 0 & 0 & 0 & 1 & 0 & 1 & 1 & 0 & 0 & 1 & 1 & 0 & 0 & 0 & 0 & 1 & 0 & 1 & 1 & $\begin{array}{ll}0 & 0\end{array}$ & $\begin{array}{ll}0 & 1\end{array}$ & 1 & 0 & 0 & 0 & 0 & 1 & 0 \\
\hline 0 & 0 & 0 & 0 & 0 & 0 & 0 & 0 & 0 & 0 & 0 & 0 & 0 & 0 & 0 & 0 & 0 & 1 & 1 & 1 & 1 & 0 & 0 & $\begin{array}{ll}0 & 0\end{array}$ & $\begin{array}{ll}0 & 0\end{array}$ & 0 & 0 & 0 & 0 & 0 & 0 & 0 \\
\hline 1 & 0 & 0 & 0 & 0 & 0 & 0 & 0 & 0 & 1 & 1 & 1 & 1 & 0 & 0 & 0 & 0 & 0 & 0 & 1 & 1 & 1 & 1 & $1 \quad 1$ & 10 & 0 & 0 & 0 & 0 & 0 & 0 & 0 \\
\hline 0 & 0 & 0 & 0 & 0 & 0 & 0 & 0 & 0 & 1 & 1 & 1 & 1 & 1 & 1 & 1 & 1 & 1 & 1 & 1 & 1 & 0 & 0 & $\begin{array}{ll}0 & 0\end{array}$ & $\begin{array}{ll}0 & 0\end{array}$ & 0 & 0 & 0 & 0 & 0 & 0 & 0 \\
\hline 0 & 0 & 0 & 0 & 0 & 1 & 1 & 1 & 1 & 0 & 0 & 0 & 0 & 0 & 0 & 0 & 0 & 0 & 0 & 0 & 0 & 0 & 0 & $\begin{array}{ll}0 & 0\end{array}$ & $\begin{array}{ll}0 & 0\end{array}$ & 0 & 0 & 0 & 1 & 1 & 1 & 1 \\
\hline 1 & 0 & 0 & 0 & 0 & 1 & 1 & 0 & 0 & 1 & 1 & 1 & 1 & 0 & 0 & 0 & 0 & 0 & 0 & 0 & 0 & 1 & 1 & 11 & 10 & 0 & 0 & 0 & 1 & 1 & 0 & 0 \\
\hline 0 & 0 & 0 & 0 & 0 & 0 & 0 & 0 & 0 & 1 & 1 & 1 & 1 & 1 & 1 & 1 & 1 & 0 & 0 & 0 & 0 & 0 & 0 & $\begin{array}{ll}0 & 0\end{array}$ & $\begin{array}{ll}0 & 0\end{array}$ & 0 & 0 & 0 & 0 & 0 & 0 & 0 \\
\hline 0 & 0 & 0 & 0 & 0 & 1 & 1 & 1 & 1 & 0 & 0 & 0 & 0 & 0 & 0 & 0 & 0 & 0 & 0 & 0 & 0 & 0 & 0 & $\begin{array}{ll}0 & 0\end{array}$ & $\begin{array}{ll}0 & 0\end{array}$ & 0 & 0 & 0 & 0 & 0 & 0 & 0 \\
\hline 1 & 0 & 1 & 0 & 1 & 0 & 1 & 1 & 0 & 0 & 1 & 0 & 1 & 0 & 1 & 0 & 1 & 0 & 0 & 0 & 0 & 0 & 1 & $\begin{array}{ll}0 & 1\end{array}$ & 10 & 1 & 0 & 1 & 0 & 0 & 0 & 0 \\
\hline 1 & 1 & 1 & 1 & 1 & 1 & 1 & 1 & 1 & 0 & 0 & 0 & 0 & 0 & 0 & 0 & 0 & 0 & 0 & 0 & 0 & 0 & 0 & $\begin{array}{lll}0 & 0\end{array}$ & $\begin{array}{ll}0 & 0\end{array}$ & 0 & 0 & 0 & 0 & 0 & 0 & 0 \\
\hline 0 & 0 & 0 & 0 & 0 & 0 & 0 & 0 & 0 & 0 & 0 & 0 & 0 & 0 & 0 & 0 & 0 & 1 & 1 & 1 & 1 & 0 & 0 & $\begin{array}{ll}0 & 0\end{array}$ & $\begin{array}{ll}0 & 0\end{array}$ & 0 & 0 & 0 & 1 & 1 & 1 & 1 \\
\hline 1 & 0 & 1 & 0 & 1 & 0 & 0 & 0 & 0 & 0 & 1 & 0 & 1 & 0 & 1 & 0 & 1 & 1 & 0 & 0 & 1 & 0 & 1 & $\begin{array}{ll}0 & 1\end{array}$ & $\begin{array}{ll}1 & 0\end{array}$ & 1 & 0 & 1 & 1 & 0 & 0 & 1 \\
\hline 1 & 1 & 1 & 1 & 1 & 0 & 0 & 0 & 0 & 0 & 0 & 0 & 0 & 0 & 0 & 0 & 0 & 0 & 0 & 0 & 0 & 0 & 0 & $\begin{array}{lll}0 & 0\end{array}$ & $\begin{array}{ll}0 & 0\end{array}$ & 0 & 0 & 0 & 0 & 0 & 0 & 0 \\
\hline 0 & 0 & 0 & 0 & 0 & 1 & 1 & 0 & 0 & 0 & 0 & 0 & 0 & 0 & 0 & 0 & 0 & 1 & 1 & 0 & 0 & 0 & 0 & $\begin{array}{ll}0 & 0\end{array}$ & $\begin{array}{ll}0 & 0\end{array}$ & 0 & 0 & 0 & 1 & 1 & 0 & 0 \\
\hline 1 & 0 & 0 & 1 & 1 & 0 & 1 & 0 & 0 & 0 & 0 & 1 & 1 & 0 & 0 & 1 & 1 & 0 & 1 & 0 & 0 & 0 & 0 & 11 & $\begin{array}{ll}1 & 0\end{array}$ & 0 & 1 & 1 & 0 & 1 & 0 & 0 \\
\hline 0 & 1 & 1 & 1 & 1 & 0 & 0 & 0 & 0 & 0 & 0 & 0 & 0 & 1 & 1 & 1 & 1 & 1 & 1 & 0 & 0 & 0 & 0 & $\begin{array}{lll}0 & 0\end{array}$ & $\begin{array}{ll}0 & 1\end{array}$ & 1 & 1 & 1 & 0 & 0 & 0 & 0 \\
\hline 0 & 0 & 0 & 0 & 0 & 0 & 0 & 1 & 1 & 0 & 0 & 0 & 0 & 0 & 0 & 0 & 0 & 0 & 0 & 1 & 1 & 0 & 0 & $\begin{array}{lll}0 & 0\end{array}$ & $\begin{array}{ll}0 & 0\end{array}$ & 0 & 0 & 0 & 0 & 0 & 1 & 1 \\
\hline 1 & 0 & 0 & 1 & 1 & 0 & 0 & 0 & 1 & 0 & 0 & 1 & 1 & 0 & 0 & 1 & 1 & 0 & 0 & 0 & 1 & 0 & 0 & 11 & 10 & 0 & 1 & 1 & 0 & 0 & 0 & 1 \\
\hline 0 & 1 & 1 & 1 & 1 & 0 & 0 & 1 & 1 & 0 & 0 & 0 & 0 & 1 & 1 & 1 & 1 & 0 & 0 & 0 & 0 & 0 & 0 & $\begin{array}{lll}0 & 0\end{array}$ & $\begin{array}{ll}0 & 1\end{array}$ & 1 & 1 & 1 & 0 & 0 & 1 & 1 \\
\hline 0 & 0 & 0 & 0 & 0 & 0 & 0 & 0 & 0 & 0 & 0 & 0 & 0 & 0 & 0 & 0 & 0 & 0 & 0 & 0 & 0 & 0 & 0 & $\begin{array}{lll}0 & 0\end{array}$ & $\begin{array}{ll}0 & 0\end{array}$ & 0 & 0 & 0 & 0 & 0 & 0 & 0 \\
\hline 1 & 1 & 1 & 1 & 1 & 0 & 0 & 0 & 0 & 0 & 0 & 0 & 0 & 0 & 0 & 0 & 0 & 0 & 0 & 0 & 0 & 1 & 1 & 11 & $\begin{array}{ll}1 & 1\end{array}$ & 1 & 1 & 1 & 0 & 0 & 0 & 0 \\
\hline 0 & 0 & 0 & 0 & 0 & 0 & 0 & 0 & 0 & 1 & 1 & 1 & 1 & 1 & 1 & 1 & 1 & 0 & 0 & 0 & 0 & 1 & 1 & 11 & $\begin{array}{ll}1 & 1\end{array}$ & 1 & 1 & 1 & 0 & 0 & 0 & 0 \\
\hline 0 & 0 & 0 & 0 & 0 & 1 & 1 & 1 & 1 & 0 & 0 & 0 & 0 & 0 & 0 & 0 & 0 & 1 & 1 & 1 & 1 & 0 & 0 & $\begin{array}{lll}0 & 0\end{array}$ & $\begin{array}{ll}0 & 0\end{array}$ & 0 & 0 & 0 & 1 & 1 & 1 & 1 \\
\hline 1 & 1 & 1 & 1 & 1 & 1 & 1 & 1 & 1 & 0 & 0 & 0 & 0 & 0 & 0 & 0 & 0 & 0 & 0 & 0 & 0 & 1 & 1 & 11 & 1 & 1 & 1 & 1 & 1 & 1 & 1 & 1 \\
\hline 0 & 0 & 0 & 0 & 0 & 0 & 0 & 0 & 0 & 1 & 1 & 1 & 1 & 1 & 1 & 1 & 1 & 1 & 1 & 1 & 1 & 1 & 1 & 1 & 1 & 1 & 1 & 1 & 1 & 1 & & \\
\hline
\end{tabular}

where the relations between bifundamentals and GLSM fields can be directly read off. 
Then we can get the total charge matrix:

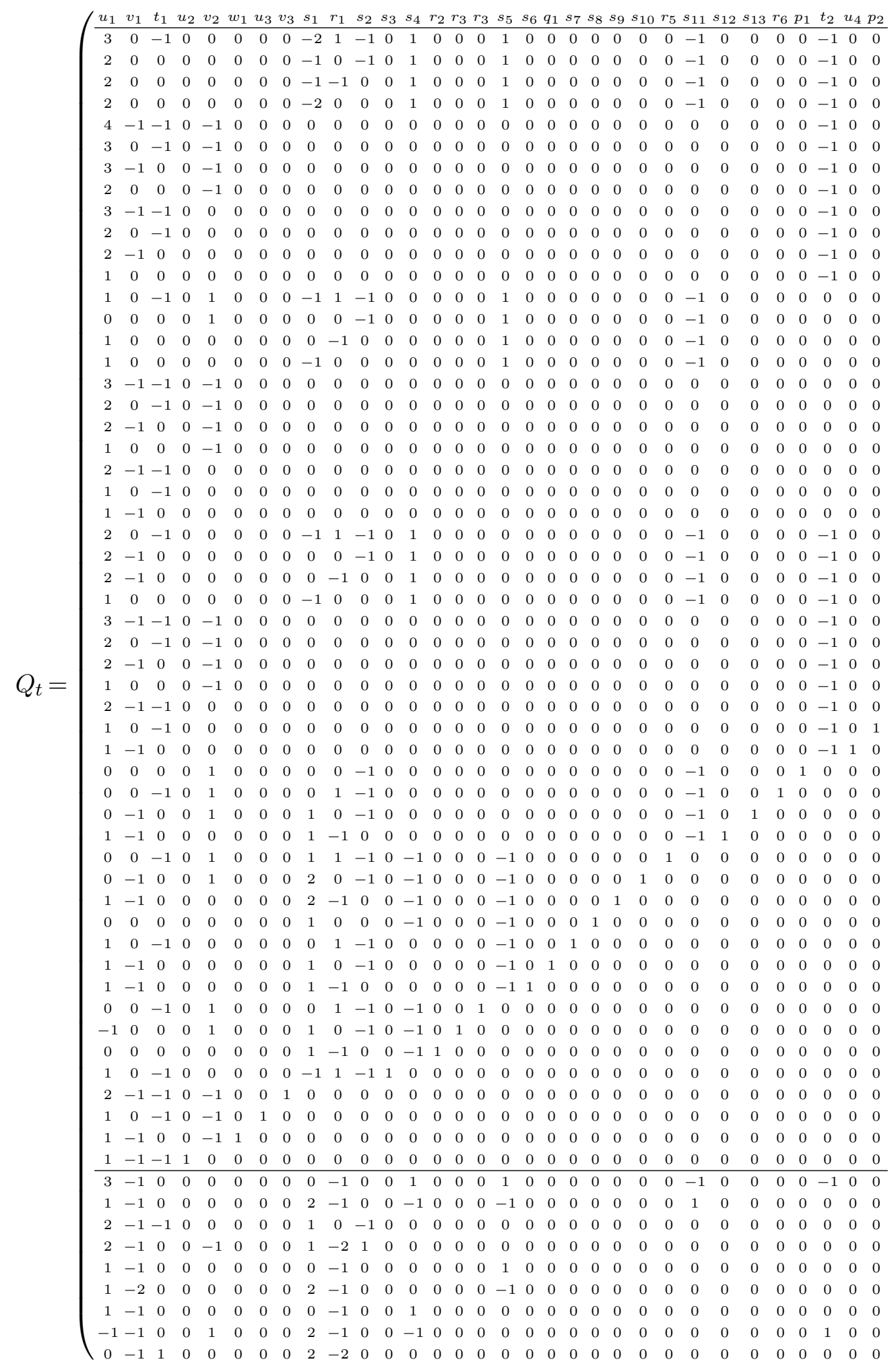



$\begin{array}{lllllllllllllllllllllllllllllllllllllll}0 & 0 & 0 & 0 & 0 & 0 & 0 & 0 & 0 & -1 & 0 & 0 & 0 & 0 & 0 & 0 & 0 & 0 & 0 & 0 & 0 & 0 & 0 & 0 & 0 & 0 & 0 & 0 & 0 & 0 & 0 & 1 & 0\end{array}$ $\begin{array}{lllllllllllllllllllllllllllllllllll}0 & 0 & 0 & 0 & 0 & 0 & 0 & 0 & 0 & -1 & 0 & 0 & 0 & 0 & 0 & 0 & 0 & 0 & 0 & 0 & 0 & 0 & 0 & 0 & 0 & 0 & 0 & 0 & 0 & 0 & 1 & 0 & 0\end{array}$ $\begin{array}{llllllllllllllllllllllllllllllllll}0 & 0 & 0 & 0 & 0 & 0 & 0 & 0 & 0 & -1 & 0 & 0 & 0 & 0 & 0 & 0 & 0 & 0 & 0 & 0 & 0 & 0 & 0 & 0 & 0 & 0 & 0 & 0 & 0 & 1 & 0 & 0 & 0\end{array}$ $\begin{array}{llllllllllllllllllllllllllllllllll}0 & 0 & 0 & 0 & 0 & 0 & 0 & 0 & 0 & -1 & 0 & 0 & 0 & 0 & 0 & 0 & 0 & 0 & 0 & 0 & 0 & 0 & 0 & 0 & 0 & 0 & 0 & 0 & 1 & 0 & 0 & 0 & 0\end{array}$ $\begin{array}{lllllllllllllllllllllllllllllllll}0 & 0 & 0 & 0 & 0 & 0 & 0 & 0 & 0 & -1 & 0 & 0 & 0 & 0 & 0 & 0 & 0 & 0 & 0 & 0 & 0 & 0 & 0 & 0 & 0 & 0 & 0 & 1 & 0 & 0 & 0 & 0 & 0\end{array}$ $\begin{array}{lllllllllllllllllllllllllllllllllll}0 & 0 & 0 & 0 & 0 & 0 & 0 & 0 & 0 & -1 & 0 & 0 & 0 & 0 & 0 & 0 & 0 & 0 & 0 & 0 & 0 & 0 & 0 & 0 & 0 & 0 & 1 & 0 & 0 & 0 & 0 & 0 & 0\end{array}$ $\begin{array}{llllllllllllllllllllllllllllllllll}0 & 0 & 0 & 0 & 0 & 0 & 0 & 0 & 0 & -1 & 0 & 0 & 0 & 0 & 0 & 0 & 0 & 0 & 0 & 0 & 0 & 0 & 0 & 0 & 0 & 1 & 0 & 0 & 0 & 0 & 0 & 0 & 0\end{array}$ $\begin{array}{llllllllllllllllllllllllllllllllll}0 & 0 & 0 & 0 & 0 & 0 & 0 & 0 & 0 & -1 & 0 & 0 & 0 & 0 & 0 & 0 & 0 & 0 & 0 & 0 & 0 & 0 & 0 & 0 & 1 & 0 & 0 & 0 & 0 & 0 & 0 & 0 & 0\end{array}$ $\begin{array}{lllllllllllllllllllllllllllllllll}0 & 0 & 0 & 0 & 0 & 0 & 0 & 0 & 0 & -1 & 0 & 0 & 0 & 0 & 0 & 0 & 0 & 0 & 0 & 0 & 0 & 0 & 0 & 1 & 0 & 0 & 0 & 0 & 0 & 0 & 0 & 0 & 0\end{array}$ $\begin{array}{llllllllllllllllllllllllllllllllll}0 & 0 & 0 & 0 & 0 & 0 & 0 & 0 & 0 & -1 & 0 & 0 & 0 & 0 & 0 & 0 & 0 & 0 & 0 & 0 & 0 & 0 & 1 & 0 & 0 & 0 & 0 & 0 & 0 & 0 & 0 & 0 & 0\end{array}$ $\begin{array}{lllllllllllllllllllllllllllllllllllllll}0 & 0 & 0 & 0 & 0 & 0 & 0 & 0 & 0 & -1 & 0 & 0 & 0 & 0 & 0 & 0 & 0 & 0 & 0 & 0 & 0 & 1 & 0 & 0 & 0 & 0 & 0 & 0 & 0 & 0 & 0 & 0 & 0\end{array}$ $\begin{array}{llllllllllllllllllllllllllllllllll}0 & 0 & 0 & 0 & 0 & 0 & 0 & 0 & 0 & -1 & 0 & 0 & 0 & 0 & 0 & 0 & 0 & 0 & 0 & 0 & 1 & 0 & 0 & 0 & 0 & 0 & 0 & 0 & 0 & 0 & 0 & 0 & 0\end{array}$ $\begin{array}{llllllllllllllllllllllllllllllllll}0 & 0 & 0 & 0 & 0 & 0 & 0 & 0 & 0 & -1 & 0 & 0 & 0 & 0 & 0 & 0 & 0 & 0 & 0 & 1 & 0 & 0 & 0 & 0 & 0 & 0 & 0 & 0 & 0 & 0 & 0 & 0 & 0\end{array}$ $\begin{array}{lllllllllllllllllllllllllllllllll}0 & 0 & 0 & 0 & 0 & 0 & 0 & 0 & 0 & -1 & 0 & 0 & 0 & 0 & 0 & 0 & 0 & 0 & 1 & 0 & 0 & 0 & 0 & 0 & 0 & 0 & 0 & 0 & 0 & 0 & 0 & 0 & 0\end{array}$ $\begin{array}{llllllllllllllllllllllllllllllllll}0 & 0 & 0 & 0 & 0 & 0 & 0 & 0 & 0 & -1 & 0 & 0 & 0 & 0 & 0 & 0 & 0 & 1 & 0 & 0 & 0 & 0 & 0 & 0 & 0 & 0 & 0 & 0 & 0 & 0 & 0 & 0 & 0\end{array}$ $\begin{array}{llllllllllllllllllllllllllllllllll}0 & 0 & 0 & 0 & 0 & 0 & 0 & 0 & 0 & -1 & 0 & 0 & 0 & 0 & 0 & 0 & 1 & 0 & 0 & 0 & 0 & 0 & 0 & 0 & 0 & 0 & 0 & 0 & 0 & 0 & 0 & 0 & 0\end{array}$ $\begin{array}{llllllllllllllllllllllllllllllllll}0 & 0 & 0 & 0 & 0 & 0 & 0 & 0 & 0 & -1 & 0 & 0 & 0 & 0 & 0 & 1 & 0 & 0 & 0 & 0 & 0 & 0 & 0 & 0 & 0 & 0 & 0 & 0 & 0 & 0 & 0 & 0 & 0\end{array}$ $\begin{array}{llllllllllllllllllllllllllllllllll}0 & 0 & 0 & 0 & 0 & 0 & 0 & 0 & 0 & -1 & 0 & 0 & 0 & 0 & 1 & 0 & 0 & 0 & 0 & 0 & 0 & 0 & 0 & 0 & 0 & 0 & 0 & 0 & 0 & 0 & 0 & 0 & 0\end{array}$ $\begin{array}{lllllllllllllllllllllllllllllllll}0 & 0 & 0 & 0 & 0 & 0 & 0 & 0 & 0 & -1 & 0 & 0 & 0 & 1 & 0 & 0 & 0 & 0 & 0 & 0 & 0 & 0 & 0 & 0 & 0 & 0 & 0 & 0 & 0 & 0 & 0 & 0 & 0\end{array}$ $\begin{array}{llllllllllllllllllllllllllllllllll}0 & 0 & 0 & 0 & 0 & 0 & 0 & 0 & 0 & -1 & 0 & 0 & 1 & 0 & 0 & 0 & 0 & 0 & 0 & 0 & 0 & 0 & 0 & 0 & 0 & 0 & 0 & 0 & 0 & 0 & 0 & 0 & 0\end{array}$ $\begin{array}{lllllllllllllllllllllllllllllllllll}0 & 0 & 0 & 0 & 0 & 0 & 0 & 0 & 0 & -1 & 0 & 1 & 0 & 0 & 0 & 0 & 0 & 0 & 0 & 0 & 0 & 0 & 0 & 0 & 0 & 0 & 0 & 0 & 0 & 0 & 0 & 0 & 0\end{array}$ $\begin{array}{llllllllllllllllllllllllllllllllll}0 & 0 & 0 & 0 & 0 & 0 & 0 & 0 & 0 & -1 & 1 & 0 & 0 & 0 & 0 & 0 & 0 & 0 & 0 & 0 & 0 & 0 & 0 & 0 & 0 & 0 & 0 & 0 & 0 & 0 & 0 & 0 & 0\end{array}$ $\begin{array}{llllllllllllllllllllllllllllllllll}0 & 0 & 0 & 0 & 0 & 0 & 0 & 0 & 1 & 0 & 0 & 0 & 0 & 0 & 0 & 0 & 0 & 0 & 0 & 0 & 0 & 0 & 0 & 0 & 0 & 0 & 0 & 0 & 0 & 0 & 0 & 0 & 0\end{array}$ $\begin{array}{lllllllllllllllllllllllllllllllll}0 & 0 & 0 & 0 & 0 & 0 & 0 & 1 & 0 & 0 & 0 & 0 & 0 & 0 & 0 & 0 & 0 & 0 & 0 & 0 & 0 & 0 & 0 & 0 & 0 & 0 & 0 & 0 & 0 & 0 & 0 & 0 & 0\end{array}$ $\begin{array}{llllllllllllllllllllllllllllllllll}0 & 0 & 0 & 0 & 0 & 0 & 1 & 0 & 0 & 0 & 0 & 0 & 0 & 0 & 0 & 0 & 0 & 0 & 0 & 0 & 0 & 0 & 0 & 0 & 0 & 0 & 0 & 0 & 0 & 0 & 0 & 0 & 0\end{array}$ $\begin{array}{lllllllllllllllllllllllllllllllllll}0 & 0 & 0 & 0 & 0 & 1 & 0 & 0 & 0 & 0 & 0 & 0 & 0 & 0 & 0 & 0 & 0 & 0 & 0 & 0 & 0 & 0 & 0 & 0 & 0 & 0 & 0 & 0 & 0 & 0 & 0 & 0 & 0\end{array}$ $\begin{array}{llllllllllllllllllllllllllllllllll}0 & 0 & 0 & 0 & 1 & 0 & 0 & 0 & 0 & 0 & 0 & 0 & 0 & 0 & 0 & 0 & 0 & 0 & 0 & 0 & 0 & 0 & 0 & 0 & 0 & 0 & 0 & 0 & 0 & 0 & 0 & 0 & 0\end{array}$ $\begin{array}{llllllllllllllllllllllllllllllllll}0 & 0 & 0 & 1 & 0 & 0 & 0 & 0 & 0 & 0 & 0 & 0 & 0 & 0 & 0 & 0 & 0 & 0 & 0 & 0 & 0 & 0 & 0 & 0 & 0 & 0 & 0 & 0 & 0 & 0 & 0 & 0 & 0\end{array}$ $\begin{array}{lllllllllllllllllllllllllllllllllll}0 & 0 & 1 & 0 & 0 & 0 & 0 & 0 & 0 & 0 & 0 & 0 & 0 & 0 & 0 & 0 & 0 & 0 & 0 & 0 & 0 & 0 & 0 & 0 & 0 & 0 & 0 & 0 & 0 & 0 & 0 & 0 & 0\end{array}$ $\begin{array}{lllllllllllllllllllllllllllllllll}0 & 1 & 0 & 0 & 0 & 0 & 0 & 0 & 0 & 0 & 0 & 0 & 0 & 0 & 0 & 0 & 0 & 0 & 0 & 0 & 0 & 0 & 0 & 0 & 0 & 0 & 0 & 0 & 0 & 0 & 0 & 0 & 0\end{array}$

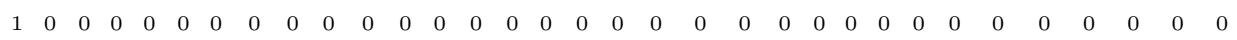
$\begin{array}{lllllllllllllllllllllllllllllllll}0 & 0 & 0 & 0 & 0 & 0 & 0 & 0 & 0 & 0 & 0 & 0 & 0 & 0 & 0 & 0 & 0 & 0 & 0 & 0 & 0 & 0 & 0 & 0 & 0 & 0 & 0 & 0 & 0 & 0 & 0 & 0 & 0\end{array}$ $\begin{array}{lllllllllllllllllllllllllllllllllllll}0 & 0 & 0 & 0 & 0 & 0 & 0 & 0 & 0 & 0 & 0 & 0 & 0 & 0 & 0 & 0 & 0 & 0 & 0 & 0 & 0 & 0 & 0 & 0 & 0 & 0 & 0 & 0 & 0 & 0 & 0 & 0 & 0\end{array}$ $\begin{array}{llllllllllllllllllllllllllllllllll}0 & 0 & 0 & 0 & 0 & 0 & 0 & 0 & 0 & 0 & 0 & 0 & 0 & 0 & 0 & 0 & 0 & 0 & 0 & 0 & 0 & 0 & 0 & 0 & 0 & 0 & 0 & 0 & 0 & 0 & 0 & 0 & 0\end{array}$ $\begin{array}{lllllllllllllllllllllllllllllllll}0 & 0 & 0 & 0 & 0 & 0 & 0 & 0 & 0 & 0 & 0 & 0 & 0 & 0 & 0 & 0 & 0 & 0 & 0 & 0 & 0 & 0 & 0 & 0 & 0 & 0 & 0 & 0 & 0 & 0 & 0 & 0 & 0\end{array}$ $\begin{array}{llllllllllllllllllllllllllllllllll}0 & 0 & 0 & 0 & 0 & 0 & 0 & 0 & 0 & 0 & 0 & 0 & 0 & 0 & 0 & 0 & 0 & 0 & 0 & 0 & 0 & 0 & 0 & 0 & 0 & 0 & 0 & 0 & 0 & 0 & 0 & 0 & 0\end{array}$ $\begin{array}{lllllllllllllllllllllllllllllllll}0 & 0 & 0 & 0 & 0 & 0 & 0 & 0 & 0 & 0 & 0 & 0 & 0 & 0 & 0 & 0 & 0 & 0 & 0 & 0 & 0 & 0 & 0 & 0 & 0 & 0 & 0 & 0 & 0 & 0 & 0 & 0 & 0\end{array}$ $\begin{array}{llllllllllllllllllllllllllllllllll}0 & 0 & 0 & 0 & 0 & 0 & 0 & 0 & 0 & 0 & 0 & 0 & 0 & 0 & 0 & 0 & 0 & 0 & 0 & 0 & 0 & 0 & 0 & 0 & 0 & 0 & 0 & 0 & 0 & 0 & 0 & 0 & 0\end{array}$ $\begin{array}{llllllllllllllllllllllllllllllllllll}0 & 0 & 0 & 0 & 0 & 0 & 0 & 0 & 0 & 0 & 0 & 0 & 0 & 0 & 0 & 0 & 0 & 0 & 0 & 0 & 0 & 0 & 0 & 0 & 0 & 0 & 0 & 0 & 0 & 0 & 0 & 0 & 0\end{array}$ $\begin{array}{lllllllllllllllllllllllllllllllll}0 & 0 & 0 & 0 & 0 & 0 & 0 & 0 & 0 & 0 & 0 & 0 & 0 & 0 & 0 & 0 & 0 & 0 & 0 & 0 & 0 & 0 & 0 & 0 & 0 & 0 & 0 & 0 & 0 & 0 & 0 & 0 & 0\end{array}$ $\begin{array}{llllllllllllllllllllllllllllllllll}0 & 0 & 0 & 0 & 0 & 0 & 0 & 0 & 0 & 0 & 0 & 0 & 0 & 0 & 0 & 0 & 0 & 0 & 0 & 0 & 0 & 0 & 0 & 0 & 0 & 0 & 0 & 0 & 0 & 0 & 0 & 0 & 0\end{array}$ $\begin{array}{lllllllllllllllllllllllllllllllll}0 & 0 & 0 & 0 & 0 & 0 & 0 & 0 & 0 & 0 & 0 & 0 & 0 & 0 & 0 & 0 & 0 & 0 & 0 & 0 & 0 & 0 & 0 & 0 & 0 & 0 & 0 & 0 & 0 & 0 & 0 & 0 & 0\end{array}$ $\begin{array}{lllllllllllllllllllllllllllllllllll}0 & 0 & 0 & 0 & 0 & 0 & 0 & 0 & 0 & 0 & 0 & 0 & 0 & 0 & 0 & 0 & 0 & 0 & 0 & 0 & 0 & 0 & 0 & 0 & 0 & 0 & 0 & 0 & 0 & 0 & 0 & 0 & 0\end{array}$ $\begin{array}{llllllllllllllllllllllllllllllllllll}0 & 0 & 0 & 0 & 0 & 0 & 0 & 0 & 0 & 0 & 0 & 0 & 0 & 0 & 0 & 0 & 0 & 0 & 0 & 0 & 0 & 0 & 0 & 0 & 0 & 0 & 0 & 0 & 0 & 0 & 0 & 0 & 0\end{array}$ $\begin{array}{lllllllllllllllllllllllllllllllll}0 & 0 & 0 & 0 & 0 & 0 & 0 & 0 & 0 & 0 & 0 & 0 & 0 & 0 & 0 & 0 & 0 & 0 & 0 & 0 & 0 & 0 & 0 & 0 & 0 & 0 & 0 & 0 & 0 & 0 & 0 & 0 & 0\end{array}$ $\begin{array}{llllllllllllllllllllllllllllllllll}0 & 0 & 0 & 0 & 0 & 0 & 0 & 0 & 0 & 0 & 0 & 0 & 0 & 0 & 0 & 0 & 0 & 0 & 0 & 0 & 0 & 0 & 0 & 0 & 0 & 0 & 0 & 0 & 0 & 0 & 0 & 0 & 0\end{array}$ $\begin{array}{llllllllllllllllllllllllllllllllll}0 & 0 & 0 & 0 & 0 & 0 & 0 & 0 & 0 & 0 & 0 & 0 & 0 & 0 & 0 & 0 & 0 & 0 & 0 & 0 & 0 & 0 & 0 & 0 & 0 & 0 & 0 & 0 & 0 & 0 & 0 & 0 & 0\end{array}$ $\begin{array}{llllllllllllllllllllllllllllllllll}0 & 0 & 0 & 0 & 0 & 0 & 0 & 0 & 0 & 0 & 0 & 0 & 0 & 0 & 0 & 0 & 0 & 0 & 0 & 0 & 0 & 0 & 0 & 0 & 0 & 0 & 0 & 0 & 0 & 0 & 0 & 0 & 0\end{array}$ $\begin{array}{llllllllllllllllllllllllllllllllllll}0 & 0 & 0 & 0 & 0 & 0 & 0 & 0 & 0 & 0 & 0 & 0 & 0 & 0 & 0 & 0 & 0 & 0 & 0 & 0 & 0 & 0 & 0 & 0 & 0 & 0 & 0 & 0 & 0 & 0 & 0 & 0 & 0\end{array}$ $\begin{array}{llllllllllllllllllllllllllllllllll}0 & 0 & 0 & 0 & 0 & 0 & 0 & 0 & 0 & 0 & 0 & 0 & 0 & 0 & 0 & 0 & 0 & 0 & 0 & 0 & 0 & 0 & 0 & 0 & 0 & 0 & 0 & 0 & 0 & 0 & 0 & 0 & 0\end{array}$ $\begin{array}{llllllllllllllllllllllllllllllllll}0 & 0 & 0 & 0 & 0 & 0 & 0 & 0 & 0 & 0 & 0 & 0 & 0 & 0 & 0 & 0 & 0 & 0 & 0 & 0 & 0 & 0 & 0 & 0 & 0 & 0 & 0 & 0 & 0 & 0 & 0 & 0 & 0\end{array}$ \begin{tabular}{ccccccccccccccccccccccccccccccccc}
0 & 0 & 0 & 0 & 0 & 0 & 0 & 0 & 0 & 0 & 0 & 0 & 0 & 0 & 0 & 0 & 0 & 0 & 0 & 0 & 0 & 0 & 0 & 0 & 0 & 0 & 0 & 0 & 0 & 0 & 0 & 0 & 0 \\
\hline 0 & 0 & 0 & 0 & 0 & 0 & 0 & 0 & 0 & -1 & 0 & 0 & 0 & 0 & 0 & 0 & 0 & 0 & 0 & 0 & 0 & 0 & 0 & 0 & 0 & 0 & 0 & 0 & 0 & 0 & 0 & 0 & 0
\end{tabular} $\begin{array}{llllllllllllllllllllllllllllllllll}0 & 0 & 0 & 0 & 0 & 0 & 0 & 0 & 0 & 0 & 0 & 0 & 0 & 0 & 0 & 0 & 0 & 0 & 0 & 0 & 0 & 0 & 0 & 0 & 0 & 0 & 0 & 0 & 0 & 0 & 0 & 0 & 0\end{array}$ $\begin{array}{llllllllllllllllllllllllllllllllll}0 & 0 & 0 & 0 & 0 & 0 & 0 & 0 & 0 & 0 & 0 & 0 & 0 & 0 & 0 & 0 & 0 & 0 & 0 & 0 & 0 & 0 & 0 & 0 & 0 & 0 & 0 & 0 & 0 & 0 & 0 & 0 & 0\end{array}$ $\begin{array}{llllllllllllllllllllllllllllllllll}0 & 0 & 0 & 0 & 0 & 0 & 0 & 0 & 0 & 0 & 0 & 0 & 0 & 0 & 0 & 0 & 0 & 0 & 0 & 0 & 0 & 0 & 0 & 0 & 0 & 0 & 0 & 0 & 0 & 0 & 0 & 0 & 0\end{array}$ $\begin{array}{llllllllllllllllllllllllllllllllllll}0 & 0 & 0 & 0 & 0 & 0 & 0 & 0 & 0 & 0 & 0 & 0 & 0 & 0 & 0 & 0 & 0 & 0 & 0 & 0 & 0 & 0 & 0 & 0 & 0 & 0 & 0 & 0 & 0 & 0 & 0 & 0 & 0\end{array}$ $\begin{array}{llllllllllllllllllllllllllllllllll}0 & 0 & 0 & 0 & 0 & 0 & 0 & 0 & 0 & 1 & 0 & 0 & 0 & 0 & 0 & 0 & 0 & 0 & 0 & 0 & 0 & 0 & 0 & 0 & 0 & 0 & 0 & 0 & 0 & 0 & 0 & 0 & 0\end{array}$ $\begin{array}{llllllllllllllllllllllllllllllllll}0 & 0 & 0 & 0 & 0 & 0 & 0 & 0 & 0 & 0 & 0 & 0 & 0 & 0 & 0 & 0 & 0 & 0 & 0 & 0 & 0 & 0 & 0 & 0 & 0 & 0 & 0 & 0 & 0 & 0 & 0 & 0 & 0\end{array}$ $\left.\begin{array}{lllllllllllllllllllllllllllllllllll}0 & 0 & 0 & 0 & 0 & 0 & 0 & 0 & 0 & 0 & 0 & 0 & 0 & 0 & 0 & 0 & 0 & 0 & 0 & 0 & 0 & 0 & 0 & 0 & 0 & 0 & 0 & 0 & 0 & 0 & 0 & 0 & 0 \\ 0 & 0 & 0 & 0 & 0 & 0 & 0 & 0 & 0 & 0 & 0 & 0 & 0 & 0 & 0 & 0 & 0 & 0 & 0 & 0 & 0 & 0 & 0 & 0 & 0 & 0 & 0 & 0 & 0 & 0 & 0 & 0 & 0\end{array}\right)$ 
with kernel

$$
\begin{aligned}
& G_{t}=\left(\begin{array}{cccccccccccccccccccccccccccccccccccccc}
u_{1} & v_{1} & t_{1} & u_{2} & v_{2} & w_{1} & u_{3} & v_{3} & s_{1} & r_{1} & s_{2} & s_{3} & s_{4} & r_{2} & r_{3} & r_{3} & s_{5} & s_{6} & q_{1} & s_{7} & s_{8} & s_{9} & s_{10} & r_{5} & s_{11} & s_{12} & s_{13} & r_{6} & p_{1} & t_{2} & u_{4} & p_{2} \\
\hline-1 & 0 & -2 & -1 & 0 & 1 & -1 & 0 & 1 & 0 & 1 & 1 & 1 & 0 & 0 & 0 & 1 & 1 & 2 & 1 & 1 & 1 & 1 & 0 & 1 & 1 & 1 & 0 & 2 & -2 & -1 & -3 \\
1 & 0 & 2 & 1 & 0 & -1 & 1 & 0 & 0 & 1 & 0 & 0 & 0 & 1 & 1 & 1 & 0 & 0 & -1 & 0 & 0 & 0 & 0 & 1 & 0 & 0 & 0 & 1 & 0 & 2 & 1 & 3 \\
1 & 1 & 1 & 1 & 1 & 1 & 1 & 1 & 1 & 1 & 1 & 1 & 1 & 1 & 1 & 1 & 1 & 1 & 1 & 1 & 1 & 1 & 1 & 1 & 1 & 1 & 1 & 1 & 1 & 1 & 1 & 1
\end{array}\right.
\end{aligned}
$$

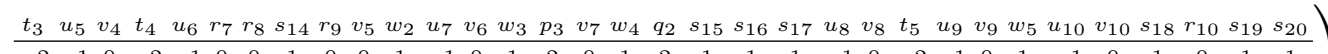

$$
\begin{aligned}
& \begin{array}{cccccccccccccccccccccccccccccccccc}
-2 & -1 & 0 & -2 & -1 & 0 & 0 & 1 & 0 & 0 & 1 & -1 & 0 & 1 & 2 & 0 & 1 & 2 & 1 & 1 & 1 & -1 & 0 & -2 & -1 & 0 & 1 & -1 & 0 & 1 & 0 & 1 & 1 \\
2 & 1 & 0 & 2 & 1 & 1 & 1 & 0 & 1 & 0 & -1 & 1 & 0 & -1 & -2 & 0 & -1 & -1 & 0 & 0 & 0 & 1 & 0 & 2 & 1 & 0 & -1 & 1 & 0 & 0 & 1 & 0 & 0 & \text {. }
\end{array} \\
& \left.\begin{array}{llllllllllllllllllllllllllllllllll}
1 & 1 & 1 & 1 & 1 & 1 & 1 & 1 & 1 & 1 & 1 & 1 & 1 & 1 & 1 & 1 & 1 & 1 & 1 & 1 & 1 & 1 & 1 & 1 & 1 & 1 & 1 & 1 & 1 & 1 & 1 & 1 & 1
\end{array}\right)
\end{aligned}
$$

From $G_{t}$, we can get the GLSM fields associated to each point as shown in (3.28), where

$$
\begin{aligned}
& q=\left\{q_{1}, q_{2}\right\}, \quad r=\left\{r_{1}, \ldots, r_{10}\right\}, \quad s=\left\{s_{1}, \ldots, s_{20}\right\}, \quad t=\left\{t_{1}, \ldots, t_{5}\right\}, \\
& u=\left\{u_{1}, \ldots, u_{10}\right\}, \quad v=\left\{v_{1}, \ldots, v_{10}\right\}, \quad w=\left\{w_{1}, \ldots, w_{5}\right\} .
\end{aligned}
$$

From $Q_{t}$ (and $Q_{F}$ ), the mesonic symmetry reads $\mathrm{U}(1)^{2} \times \mathrm{U}(1)_{\mathrm{R}}$ and the baryonic symmetry reads $\mathrm{U}(1)_{\mathrm{h}}^{4} \times \mathrm{U}(1)^{5}$, where the subscripts " $\mathrm{R}$ " and " $\mathrm{h}$ " indicate $\mathrm{R}$ - and hidden symmetries respectively.

The Hilbert series of the toric cone is

$$
\begin{aligned}
H S= & \frac{1}{\left(1-\frac{t_{1} t_{2}}{t_{3}}\right)\left(1-\frac{t_{1} t_{2}^{2}}{t_{3}}\right)\left(1-\frac{t_{3}^{3}}{t_{1}^{2} t_{2}^{3}}\right)}+\frac{1}{\left(1-t_{2} t_{3}\right)\left(1-\frac{t_{1} t_{2}}{t_{3}}\right)\left(1-\frac{t_{3}}{t_{1} t_{2}^{2}}\right)} \\
& +\frac{1}{\left(1-t_{1}\right)\left(1-t_{2}\right)\left(1-\frac{t_{3}}{t_{1} t_{2}}\right)}+\frac{1}{\left(1-\frac{1}{t_{1}}\right)\left(1-t_{2}\right)\left(1-\frac{t_{1} t_{3}}{t_{2}}\right)} \\
& +\frac{1}{\left(1-\frac{1}{t_{1}}\right)\left(1-\frac{t_{1}}{t_{2}}\right)\left(1-t_{2} t_{3}\right)}+\frac{1}{\left(1-\frac{t_{3}}{t_{1}}\right)\left(1-t_{2} t_{3}\right)\left(1-\frac{t_{1}}{t_{2} t_{3}}\right)} \\
& +\frac{1}{\left(1-\frac{t_{1}}{t_{3}}\right)\left(1-t_{2} t_{3}\right)\left(1-\frac{t_{3}}{t_{1} t_{2}}\right)}+\frac{1}{\left(1-\frac{1}{t_{1} t_{3}}\right)\left(1-t_{2} t_{3}\right)\left(1-\frac{t_{1} t_{3}}{t_{2}}\right)} \\
& +\frac{1}{\left(1-t_{1}\right)\left(1-\frac{1}{t_{2}}\right)\left(1-\frac{t_{2} t_{3}}{t_{1}}\right)}+\frac{1}{\left(1-\frac{1}{t_{2}}\right)\left(1-\frac{t_{2}}{t_{1}}\right)\left(1-t_{1} t_{3}\right)} .
\end{aligned}
$$

The volume function is then

$$
V=\frac{10}{\left(b_{2}+3\right)\left(-b_{1}+b_{2}-3\right)\left(2 b_{1}+3 b_{2}-9\right)} .
$$

Minimizing $V$ yields $V_{\min }=1 / 10$ at $b_{1}=1, b_{2}=-1$. Thus, $a_{\max }=5 / 2$. Together with the superconformal conditions, we can solve for the R-charges of the bifundamentals, which are $X_{I}=2 / 3$ for any $I$, viz, for all the bifundamentals. Hence, the R-charges of GLSM fields are $p_{i}=2 / 3$ with others vanishing.

\subsection{Polytope 5: $\mathbb{C}^{3} /\left(\mathbb{Z}_{2} \times \mathbb{Z}_{6}\right)(1,0,1)(1,0,5)$}

The polytope is

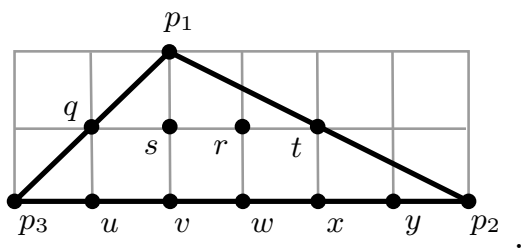


The brane tiling and the corresponding quiver are
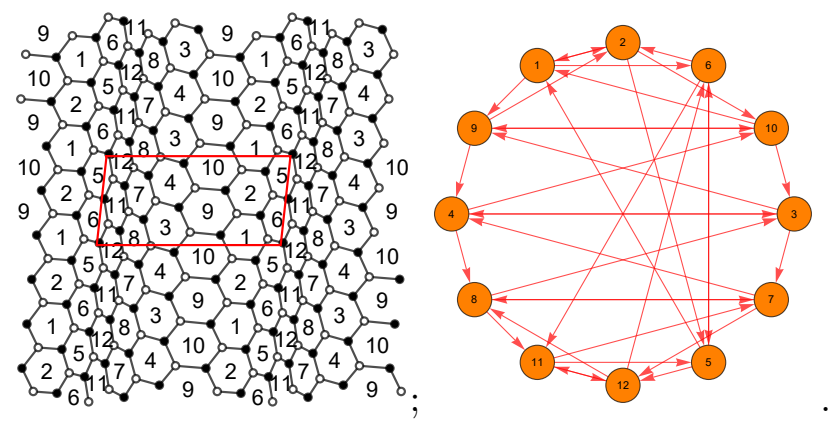

The superpotential is

$$
\begin{aligned}
W= & X_{1,9} X_{9,2} X_{2,1}+X_{2,10} X_{10,1} X_{1,2}+X_{10,3} X_{3,9} X_{9,10}+X_{9,4} X_{4,10} X_{10,9} \\
& +X_{3,7} X_{7,4} X_{4,3}+X_{4,8} X_{8,3} X_{3,4}+X_{7,12} X_{12,8} X_{8,7}+X_{8,11} X_{11,7} X_{7,8} \\
& +X_{11,5} X_{5,12} X_{12,11}+X_{12,6} X_{6,11} X_{11,12}+X_{5,1} X_{1,6} X_{6,5}+X_{6,2} X_{2,5} X_{5,6} \\
& -X_{1,9} X_{9,10} X_{10,1}-X_{2,10} X_{10,9} X_{9,2}-X_{3,9} X_{9,4} X_{4,3}-X_{4,10} X_{10,3} X_{3,4} \\
& -X_{8,3} X_{3,7} X_{7,8}-X_{7,4} X_{4,8} X_{8,7}-X_{11,7} X_{7,12} X_{12,11}-X_{12,8} X_{8,11} X_{11,12} \\
& -X_{5,12} X_{12,6} X_{6,5}-X_{6,11} X_{11,5} X_{5,6}-X_{2,5} X_{5,1} X_{1,2}-X_{1,6} X_{6,2} X_{2,1}
\end{aligned}
$$

The number of perfect matchings is $c=129$, which leads to gigantic $P, Q_{t}$ and $G_{t}$. Hence, we will not list them here. The GLSM fields associated to each point are shown in (3.37), where

$$
\begin{aligned}
& q=\left\{q_{1}, q_{2}\right\}, \quad r=\left\{r_{1}, \ldots, r_{30}\right\}, \quad u=\left\{u_{1}, \ldots, u_{6}\right\}, \quad v=\left\{v_{1}, v_{15}\right\}, \quad w=\left\{w_{1}, \ldots, w_{20}\right\}, \\
& t=\left\{t_{1}, t_{2}\right\}, \quad s=\left\{s_{1}, \ldots, s_{30}\right\}, \quad y=\left\{y_{1}, \ldots, y_{6}\right\}, \quad x=\left\{x_{1}, \ldots, x_{15}\right\} .
\end{aligned}
$$

The mesonic symmetry reads $\mathrm{U}(1)^{2} \times \mathrm{U}(1)_{\mathrm{R}}$ and the baryonic symmetry reads $\mathrm{U}(1)_{\mathrm{h}}^{4} \times \mathrm{U}(1)^{7}$, where the subscripts "R" and " $\mathrm{h}$ " indicate R- and hidden symmetries respectively.

The Hilbert series of the toric cone is

$$
\begin{aligned}
H S= & \frac{1}{\left(1-t_{2}\right)\left(1-\frac{t_{1} t_{2}}{t_{3}}\right)\left(1-\frac{t_{3}^{2}}{t_{1} t_{2}^{2}}\right)}+\frac{1}{\left(1-t_{2} t_{3}\right)\left(1-\frac{t_{1} t_{2}}{t_{3}^{2}}\right)\left(1-\frac{t_{3}^{2}}{t_{1} t_{2}^{2}}\right)} \\
& +\frac{1}{\left(1-\frac{1}{t_{2}}\right)\left(1-\frac{t_{3}^{2}}{t_{1}}\right)\left(1-\frac{t_{1} t_{2}}{t_{3}}\right)}+\frac{1}{\left(1-\frac{t_{1}}{t_{3}^{2}}\right)\left(1-t_{2} t_{3}\right)\left(1-\frac{t_{3}^{2}}{t_{1} t_{2}}\right)} \\
& +\frac{1}{\left(1-t_{1}\right)\left(1-t_{2}\right)\left(1-\frac{t_{3}}{t_{1} t_{2}}\right)}+\frac{1}{\left(1-\frac{1}{t_{1}}\right)\left(1-t_{2}\right)\left(1-\frac{t_{1} t_{3}}{t_{2}}\right)} \\
& +\frac{1}{\left(1-t_{1}\right)\left(1-\frac{1}{t_{1} t_{2}}\right)\left(1-t_{2} t_{3}\right)}+\frac{1}{\left(1-t_{1} t_{3}\right)\left(1-t_{2} t_{3}\right)\left(1-\frac{1}{t_{1} t_{2} t_{3}}\right)} \\
& +\frac{1}{\left(1-\frac{t_{1}}{t_{3}}\right)\left(1-t_{2} t_{3}\right)\left(1-\frac{t_{3}}{t_{1} t_{2}}\right)}+\frac{1}{\left(1-\frac{1}{t_{1} t_{3}}\right)\left(1-t_{2} t_{3}\right)\left(1-\frac{t_{1} t_{3}}{t_{2}}\right)} \\
& +\frac{1}{\left(1-\frac{1}{t_{1}}\right)\left(1-\frac{1}{t_{2}}\right)\left(1-t_{1} t_{2} t_{3}\right)}+\frac{1}{\left(1-\frac{1}{t_{2}}\right)\left(1-t_{1} t_{2}\right)\left(1-\frac{t_{3}}{t_{1}}\right)} .
\end{aligned}
$$


The volume function is then

$$
V=\frac{6}{\left(b_{2}+3\right)\left(-b_{1}+b_{2}-3\right)\left(b_{1}+2 b_{2}-6\right)} .
$$

Minimizing $V$ yields $V_{\min }=1 / 12$ at $b_{1}=2, b_{2}=-1$. Thus, $a_{\max }=3$. Together with the superconformal conditions, we can solve for the R-charges of the bifundamentals, which are $X_{I}=2 / 3$ for any $I$, viz, for all the bifundamentals. Hence, the R-charges of GLSM fields are $p_{i}=2 / 3$ with others vanishing. We find that all the triangles can give the same R-charge vectors.

\section{Nineteen quadrilaterals}

Now moving on to quadrilaterals, we should recall that each polytope in section 4-6 corresponds to more than one dimer models and toric quivers. In the main context, we will just list one for each polytope.

\subsection{Polytope 6: $L^{3,3,1}$}

The polytope is

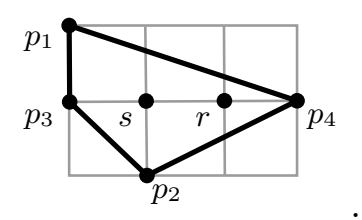

The brane tiling are the corresponding quiver are

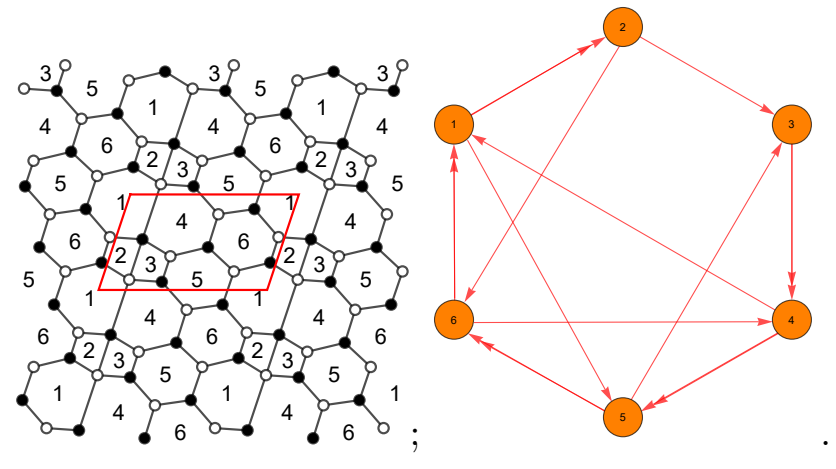

The superpotential is

$$
\begin{aligned}
W= & X_{15} X_{56}^{1} X_{61}^{2}+X_{26} X_{61}^{1} X_{12}^{2}+X_{64} X_{45}^{1} X_{56}^{2}+X_{53} X_{34}^{1} X_{45}^{2}+X_{12}^{1} X_{23} X_{34}^{2} X_{41} \\
& -X_{61}^{2} X_{12}^{1} X_{26}-X_{56}^{2} X_{61}^{1} X_{15}-X_{45}^{2} X_{56}^{1} X_{64}-X_{34}^{2} X_{45}^{1} X_{53}-X_{23} X_{34}^{1} X_{41} X_{12}^{2}
\end{aligned}
$$


The perfect matching matrix is

$$
P=\left(\begin{array}{c|cccccccccccccccccc} 
& s_{1} & s_{2} & r_{1} & s_{3} & s_{4} & s_{5} & r_{2} & p_{1} & p_{2} & p_{3} & s_{6} & r_{3} & r_{4} & r_{5} & p_{4} & s_{7} & s_{8} & r_{6} \\
\hline X_{12}^{1} & 1 & 0 & 0 & 0 & 1 & 0 & 0 & 1 & 0 & 0 & 0 & 0 & 1 & 0 & 0 & 0 & 0 & 0 \\
X_{12}^{2} & 1 & 0 & 0 & 0 & 1 & 0 & 0 & 0 & 1 & 0 & 0 & 0 & 1 & 0 & 0 & 0 & 0 & 0 \\
X_{15} & 1 & 1 & 1 & 0 & 0 & 0 & 0 & 0 & 0 & 0 & 0 & 1 & 1 & 1 & 1 & 0 & 0 & 0 \\
X_{23} & 0 & 1 & 0 & 0 & 0 & 1 & 0 & 0 & 0 & 1 & 0 & 0 & 0 & 1 & 0 & 0 & 1 & 0 \\
X_{26} & 0 & 1 & 1 & 1 & 0 & 1 & 1 & 0 & 0 & 0 & 0 & 1 & 0 & 1 & 1 & 0 & 0 & 0 \\
X_{34}^{1} & 0 & 0 & 0 & 1 & 0 & 0 & 0 & 1 & 0 & 0 & 0 & 1 & 0 & 0 & 0 & 1 & 0 & 0 \\
X_{34}^{2} & 0 & 0 & 0 & 1 & 0 & 0 & 0 & 0 & 1 & 0 & 0 & 1 & 0 & 0 & 0 & 1 & 0 & 0 \\
X_{45}^{1} & 1 & 1 & 1 & 0 & 0 & 0 & 0 & 1 & 0 & 1 & 1 & 0 & 0 & 0 & 0 & 0 & 0 & 0 \\
X_{45}^{2} & 1 & 1 & 1 & 0 & 0 & 0 & 0 & 0 & 1 & 1 & 1 & 0 & 0 & 0 & 0 & 0 & 0 & 0 \\
X_{41} & 0 & 0 & 1 & 0 & 0 & 0 & 1 & 0 & 0 & 0 & 1 & 0 & 0 & 0 & 1 & 0 & 0 & 1 \\
X_{56}^{1} & 0 & 0 & 0 & 1 & 1 & 1 & 1 & 1 & 0 & 0 & 0 & 0 & 0 & 0 & 0 & 0 & 0 & 0 \\
X_{56}^{2} & 0 & 0 & 0 & 1 & 1 & 1 & 1 & 0 & 1 & 0 & 0 & 0 & 0 & 0 & 0 & 0 & 0 & 0 \\
X_{53} & 0 & 0 & 0 & 0 & 1 & 1 & 1 & 0 & 0 & 0 & 0 & 0 & 1 & 1 & 1 & 0 & 1 & 1 \\
X_{61}^{1} & 0 & 0 & 0 & 0 & 0 & 0 & 0 & 1 & 0 & 1 & 1 & 0 & 0 & 0 & 0 & 1 & 1 & 1 \\
X_{61}^{2} & 0 & 0 & 0 & 0 & 0 & 0 & 0 & 0 & 1 & 1 & 1 & 0 & 0 & 0 & 0 & 1 & 1 & 1 \\
X_{64} & 0 & 0 & 0 & 0 & 0 & 0 & 0 & 0 & 0 & 0 & 0 & 1 & 1 & 1 & 1 & 1 & 1 & 1
\end{array}\right),
$$

where the relations between bifundamentals and GLSM fields can be directly read off. Then we can get the total charge matrix:

$$
Q_{t}=\left(\begin{array}{cccccccccccccccccc}
s_{1} & s_{2} & r_{1} & s_{3} & s_{4} & s_{5} & r_{2} & p_{1} & p_{2} & p_{3} & s_{6} & r_{3} & r_{4} & r_{5} & p_{4} & s_{7} & s_{8} & r_{6} \\
\hline 2 & 0 & -1 & 2 & -1 & 0 & 0 & -1 & -1 & 0 & 0 & -1 & 0 & 0 & 0 & 0 & 0 & 1 \\
2 & -1 & 0 & 2 & -1 & 0 & 0 & -1 & -1 & 0 & 0 & -1 & 0 & 0 & 0 & 0 & 1 & 0 \\
1 & 0 & 0 & 1 & 0 & 0 & 0 & -1 & -1 & 0 & 0 & -1 & 0 & 0 & 0 & 1 & 0 & 0 \\
1 & 0 & -1 & 1 & -1 & 0 & 0 & 0 & 0 & 0 & 0 & -1 & 0 & 0 & 1 & 0 & 0 & 0 \\
1 & -1 & 0 & 1 & -1 & 0 & 0 & 0 & 0 & 0 & 0 & -1 & 0 & 1 & 0 & 0 & 0 & 0 \\
0 & 0 & 0 & 1 & -1 & 0 & 0 & 0 & 0 & 0 & 0 & -1 & 1 & 0 & 0 & 0 & 0 & 0 \\
1 & 0 & -1 & 1 & 0 & 0 & 0 & -1 & -1 & 0 & 1 & 0 & 0 & 0 & 0 & 0 & 0 & 0 \\
1 & -1 & 0 & 1 & 0 & 0 & 0 & -1 & -1 & 1 & 0 & 0 & 0 & 0 & 0 & 0 & 0 & 0 \\
1 & 0 & -1 & 0 & -1 & 0 & 1 & 0 & 0 & 0 & 0 & 0 & 0 & 0 & 0 & 0 & 0 & 0 \\
1 & -1 & 0 & 0 & -1 & 1 & 0 & 0 & 0 & 0 & 0 & 0 & 0 & 0 & 0 & 0 & 0 & 0 \\
\hline 3 & 0 & -1 & 3 & 0 & 0 & 0 & -2 & -2 & 0 & 0 & -1 & 0 & 0 & 0 & 0 & 0 & 0 \\
3 & 0 & -1 & 1 & -1 & 0 & 0 & -1 & -1 & 0 & 0 & 0 & 0 & 0 & 0 & 0 & 0 & 0 \\
2 & 0 & -2 & 1 & 0 & 0 & 0 & -1 & -1 & 0 & 0 & 1 & 0 & 0 & 0 & 0 & 0 & 0 \\
1 & 1 & -1 & 0 & 1 & 0 & 0 & -1 & -1 & 0 & 0 & 0 & 0 & 0 & 0 & 0 & 0 & 0 \\
3 & -1 & -1 & 1 & 0 & 0 & 0 & -1 & -1 & 0 & 0 & 0 & 0 & 0 & 0 & 0 & 0 & 0
\end{array}\right)
$$

with kernel

$$
G_{t}=\left(\begin{array}{cccccccccccccccccc}
s_{1} & s_{2} & r_{1} & s_{3} & s_{4} & s_{5} & r_{2} & p_{1} & p_{2} & p_{3} & s_{6} & r_{3} & r_{4} & r_{5} & p_{4} & s_{7} & s_{8} & r_{6} \\
\hline 0 & 0 & 1 & 0 & 0 & 0 & 1 & -1 & 0 & -1 & 0 & 1 & 1 & 1 & 2 & 0 & 0 & 1 \\
1 & 1 & 0 & 1 & 1 & 1 & 0 & 3 & 0 & 2 & 1 & 0 & 0 & 0 & -1 & 1 & 1 & 0 \\
1 & 1 & 1 & 1 & 1 & 1 & 1 & 1 & 1 & 1 & 1 & 1 & 1 & 1 & 1 & 1 & 1 & 1
\end{array}\right) .
$$

From $G_{t}$, we can get the GLSM fields associated to each point as shown in (4.1), where

$$
r=\left\{r_{1}, \ldots, r_{6}\right\}, s=\left\{s_{1}, \ldots, s_{8}\right\} .
$$

From $Q_{t}$ (and $Q_{F}$ ), the mesonic symmetry reads $\mathrm{SU}(2) \times \mathrm{U}(1) \times \mathrm{U}(1)_{\mathrm{R}}$ and the baryonic symmetry reads $\mathrm{U}(1)_{\mathrm{h}}^{4} \times \mathrm{U}(1)$, where the subscripts " $\mathrm{R}$ " and " $\mathrm{h}$ " indicate $\mathrm{R}$ - and hidden symmetries respectively.

The Hilbert series of the toric cone is

$$
\begin{aligned}
H S= & \frac{1}{\left(1-t_{2}\right)\left(1-\frac{t_{1}}{t_{2}^{2} t_{3}{ }^{2}}\right)\left(1-\frac{t_{2} t_{3}}{t_{1}}\right)}+\frac{1}{\left(1-\frac{1}{t_{2}}\right)\left(1-\frac{1}{t_{1} t_{2}}\right)\left(1-\frac{t_{1} t_{2} t_{3}}{t_{3}}\right)} \\
& +\frac{1}{\left(1-\frac{1}{t_{2}}\right)\left(1-\frac{t_{1} t_{2} 3}{t_{3}{ }^{2}}\right)\left(1-\frac{t_{3}}{t_{1} t_{2}{ }^{2}}\right)}+\frac{1}{\left(1-\frac{1}{t_{1}}\right)\left(1-t_{2}\right)\left(1-\frac{t_{1}}{t_{2} t_{3}}\right)} \\
& +\frac{1}{\left(1-\frac{1}{t_{2}}\right)\left(1-t_{1} t_{2}\right)\left(1-\frac{1}{t_{1} t_{3}}\right)}+\frac{1}{\left(1-t_{1}\right)\left(1-t_{2}\right)\left(1-\frac{1}{t_{1} t_{2} t_{3}}\right)} .
\end{aligned}
$$


The volume function is then

$$
V=\frac{3\left(4 b_{1}+2 b_{2}+21\right)}{\left(b_{1}+3\right)\left(b_{1}+b_{2}+3\right)\left(b_{1}+3 b_{2}-6\right)\left(b_{1}-2\left(b_{2}+3\right)\right)} .
$$

Minimizing $V$ yields $V_{\min }=\frac{4}{405}(9+4 \sqrt{6})$ at $b_{1}=(-6+3 \sqrt{6}) / 2, b_{2}=0$. Thus, $a_{\max }=$ $\frac{27}{16}(-9+4 \sqrt{6})$. Together with the superconformal conditions, we can solve for the R-charges of the bifundamentals. Then the R-charges of GLSM fields should satisfy

$$
\begin{gathered}
\left(p_{3}+5 p_{4}\right) p_{2}^{2}+\left(p_{3}^{2}+6 p_{4} p_{3}-2 p_{3}+5 p_{4}^{2}-10 p_{4}\right) p_{2} \\
=-3 p_{4} p_{3}^{2}-3 p_{4}^{2} p_{3}+6 p_{4} p_{3}-8 \sqrt{6}+18
\end{gathered}
$$

constrained by $\sum_{i=1}^{4} p_{i}=2$ and $0<p_{i}<2$, with others vanishing.

\subsection{Polytope 7: $L^{3,3,2}$}

The polytope is

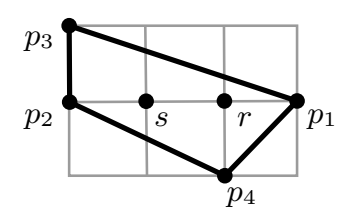

The brane tiling and the corresponding quiver are
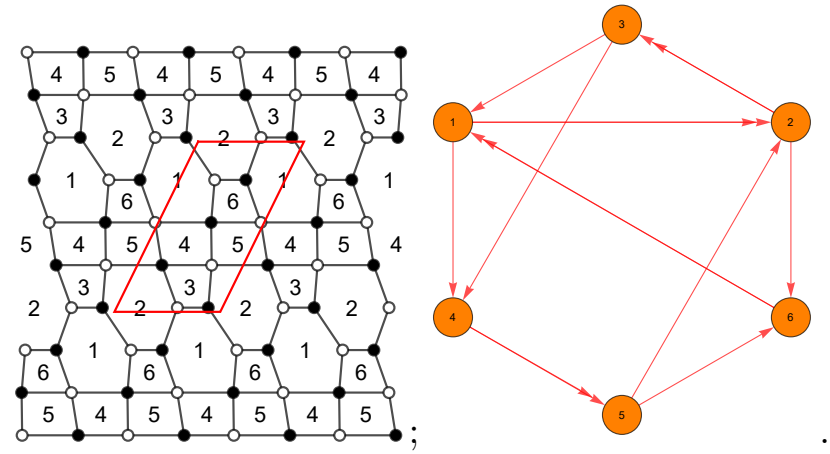

The superpotential is

$$
\begin{aligned}
W= & X_{12}^{1} X_{26} X_{61}^{2}+X_{12}^{2} X_{23}^{1} X_{31}+X_{14} X_{45}^{2} X_{56} X_{61}^{1}+X_{34} X_{45}^{1} X_{52} X_{23}^{2} \\
& -X_{12}^{2} X_{26} X_{61}^{1}-X_{12}^{1} X_{23}^{2} X_{31}-X_{14} X_{45}^{1} X_{56} X_{61}^{2}-X_{34} X_{45}^{2} X_{52} X_{23}^{1} .
\end{aligned}
$$

The perfect matching matrix is

$$
P=\left(\begin{array}{c|ccccccccccccccccc} 
& r_{1} & s_{1} & r_{2} & s_{2} & r_{3} & r_{4} & p_{1} & s_{3} & p_{2} & s_{4} & s_{5} & r_{5} & p_{3} & p_{4} & s_{6} & s_{7} & r_{6} \\
\hline X_{12}^{1} & 0 & 1 & 0 & 1 & 0 & 1 & 0 & 0 & 1 & 0 & 1 & 0 & 1 & 0 & 0 & 0 & 0 \\
X_{12}^{2} & 0 & 1 & 0 & 1 & 0 & 1 & 0 & 0 & 1 & 0 & 1 & 0 & 0 & 1 & 0 & 0 & 0 \\
X_{14} & 1 & 1 & 1 & 0 & 0 & 1 & 1 & 0 & 0 & 0 & 0 & 0 & 0 & 0 & 0 & 0 & 0 \\
X_{23}^{1} & 1 & 0 & 0 & 0 & 0 & 0 & 0 & 1 & 0 & 0 & 0 & 0 & 1 & 0 & 1 & 0 & 0 \\
X_{23}^{2} & 1 & 0 & 0 & 0 & 0 & 0 & 0 & 1 & 0 & 0 & 0 & 0 & 0 & 1 & 1 & 0 & 0 \\
X_{26} & 1 & 0 & 1 & 0 & 1 & 0 & 1 & 1 & 0 & 1 & 0 & 1 & 0 & 0 & 0 & 0 & 0 \\
X_{31} & 0 & 0 & 1 & 0 & 1 & 0 & 1 & 0 & 0 & 1 & 0 & 1 & 0 & 0 & 0 & 1 & 1 \\
X_{34} & 0 & 1 & 1 & 0 & 0 & 0 & 0 & 0 & 1 & 1 & 0 & 0 & 0 & 0 & 0 & 1 & 0 \\
X_{45}^{1} & 0 & 0 & 0 & 1 & 1 & 0 & 0 & 0 & 0 & 0 & 0 & 0 & 1 & 0 & 0 & 0 & 0 \\
X_{45}^{2} & 0 & 0 & 0 & 1 & 1 & 0 & 0 & 0 & 0 & 0 & 0 & 0 & 0 & 1 & 0 & 0 & 0 \\
X_{52} & 0 & 0 & 0 & 0 & 0 & 1 & 1 & 0 & 0 & 0 & 1 & 1 & 0 & 0 & 0 & 0 & 1 \\
X_{56} & 0 & 0 & 0 & 0 & 0 & 0 & 0 & 1 & 1 & 1 & 1 & 1 & 0 & 0 & 0 & 0 & 0 \\
X_{61}^{1} & 0 & 0 & 0 & 0 & 0 & 0 & 0 & 0 & 0 & 0 & 0 & 0 & 1 & 0 & 1 & 1 & 1 \\
X_{61}^{2} & 0 & 0 & 0 & 0 & 0 & 0 & 0 & 0 & 0 & 0 & 0 & 0 & 0 & 1 & 1 & 1 & 1
\end{array}\right),
$$


where the relations between bifundamentals and GLSM fields can be directly read off. Then we can get the total charge matrix:

$$
Q_{t}=\left(\begin{array}{ccccccccccccccccc}
r_{1} & s_{1} & r_{2} & s_{2} & r_{3} & r_{4} & p_{1} & s_{3} & p_{2} & s_{4} & s_{5} & r_{5} & p_{3} & p_{4} & s_{6} & s_{7} & r_{6} \\
\hline 1 & 1 & -1 & 1 & 0 & -1 & 0 & 0 & 0 & 0 & 0 & 0 & -1 & -1 & 0 & 0 & 1 \\
1 & 0 & -1 & 1 & 0 & 0 & 0 & 0 & 0 & 0 & 0 & 0 & -1 & -1 & 0 & 1 & 0 \\
0 & 0 & 0 & 1 & 0 & 0 & 0 & 0 & 0 & 0 & 0 & 0 & -1 & -1 & 1 & 0 & 0 \\
1 & 1 & -1 & 0 & 0 & -1 & 0 & -1 & 0 & 0 & 0 & 1 & 0 & 0 & 0 & 0 & 0 \\
1 & 0 & 0 & 0 & 0 & -1 & 0 & -1 & 0 & 0 & 1 & 0 & 0 & 0 & 0 & 0 & 0 \\
1 & 0 & -1 & 0 & 0 & 0 & 0 & -1 & 0 & 1 & 0 & 0 & 0 & 0 & 0 & 0 & 0 \\
1 & -1 & 0 & 0 & 0 & 0 & 0 & -1 & 1 & 0 & 0 & 0 & 0 & 0 & 0 & 0 & 0 \\
0 & 1 & -1 & 0 & 0 & -1 & 1 & 0 & 0 & 0 & 0 & 0 & 0 & 0 & 0 & 0 & 0 \\
0 & 1 & -1 & -1 & 1 & 0 & 0 & 0 & 0 & 0 & 0 & 0 & 0 & 0 & 0 & 0 & 0 \\
\hline 1 & 1 & -1 & 2 & 0 & 0 & 0 & 1 & 0 & 0 & 0 & 0 & -2 & -2 & 0 & 0 & 0 \\
2 & 1 & -1 & 2 & 0 & -1 & 0 & -1 & 0 & 0 & 0 & 0 & -1 & -1 & 0 & 0 & 0 \\
1 & 2 & -1 & 0 & 0 & 0 & 0 & 0 & 0 & 0 & 0 & 0 & -1 & -1 & 0 & 0 & 0 \\
2 & 1 & -2 & 1 & 0 & 0 & 0 & 0 & 0 & 0 & 0 & 0 & -1 & -1 & 0 & 0 & 0 \\
0 & 1 & -1 & 1 & 0 & 1 & 0 & 0 & 0 & 0 & 0 & 0 & -1 & -1 & 0 & 0 & 0
\end{array}\right)
$$

with kernel

$$
G_{t}=\left(\begin{array}{ccccccccccccccccc}
r_{1} & s_{1} & r_{2} & s_{2} & r_{3} & r_{4} & p_{1} & s_{3} & p_{2} & s_{4} & s_{5} & r_{5} & p_{3} & p_{4} & s_{6} & s_{7} & r_{6} \\
\hline 1 & 0 & 1 & 0 & 1 & 1 & 2 & 0 & -1 & 0 & 0 & 1 & 0 & 0 & 0 & 0 & 1 \\
0 & 1 & 0 & 1 & 0 & 0 & -1 & 1 & 2 & 1 & 1 & 0 & 2 & 0 & 1 & 1 & 0 \\
1 & 1 & 1 & 1 & 1 & 1 & 1 & 1 & 1 & 1 & 1 & 1 & 1 & 1 & 1 & 1 & 1
\end{array}\right) .
$$

From $G_{t}$, we can get the GLSM fields associated to each point as shown in (4.11), where

$$
r=\left\{r_{1}, \ldots, r_{6}\right\}, s=\left\{s_{1}, \ldots, s_{7}\right\} .
$$

From $Q_{t}$ (and $Q_{F}$ ), the mesonic symmetry reads $\mathrm{SU}(2) \times \mathrm{U}(1) \times \mathrm{U}(1)_{\mathrm{R}}$ and the baryonic symmetry reads $\mathrm{U}(1)_{\mathrm{h}}^{4} \times \mathrm{U}(1)$, where the subscripts " $\mathrm{R}$ " and " $\mathrm{h}$ " indicate $\mathrm{R}$ - and hidden symmetries respectively.

The Hilbert series of the toric cone is

$$
\begin{aligned}
H S= & \frac{1}{\left(1-t_{2}\right)\left(1-t_{1} t_{2}\right)\left(1-\frac{t_{3}}{t_{1} t_{2}^{2}}\right)}+\frac{1}{\left(1-\frac{1}{t_{2}}\right)\left(1-\frac{1}{t_{1} t_{2}}\right)\left(1-t_{1} t_{2}^{2} t_{3}\right)} \\
& +\frac{1}{\left(1-t_{2}\right)\left(1-\frac{t_{1} t_{2}^{2}}{t_{3}}\right)\left(1-\frac{t_{3}^{2}}{t_{1} t_{2}^{3}}\right)}+\frac{1}{\left(1-\frac{1}{t_{2}}\right)\left(1-\frac{t_{1}}{t_{3}}\right)\left(1-\frac{t_{2} t_{3}^{2}}{t_{1}}\right)} \\
& +\frac{1}{\left(1-t_{2}\right)\left(1-\frac{1}{t_{1} t_{2}}\right)\left(1-t_{1} t_{3}\right)}+\frac{1}{\left(1-\frac{1}{t_{2}}\right)\left(1-t_{1} t_{2}\right)\left(1-\frac{t_{3}}{t_{1}}\right)} .
\end{aligned}
$$

The volume function is then

$$
V=\frac{6\left(b_{1}+b_{2}+12\right)}{\left(b_{1}+3\right)\left(b_{1}-b_{2}-6\right)\left(b_{1}+2 b_{2}+3\right)\left(b_{1}+3 b_{2}-6\right)} .
$$

Minimizing $V$ yields $V_{\min }=\frac{1}{648}(63+11 \sqrt{33})$ at $b_{1}=\frac{3}{2}(-5+\sqrt{33}), b_{2}=0$. Thus, $a_{\max }=\frac{1}{4}(-1701+297 \sqrt{33})$. Together with the superconformal conditions, we can solve for the R-charges of the bifundamentals. Then the R-charges of GLSM fields should satisfy

$$
\begin{aligned}
\left(3 p_{3}+3 p_{4}\right) p_{2}^{2} & +\left(3 p_{3}^{2}+8 p_{4} p_{3}-6 p_{3}+3 p_{4}^{2}-6 p_{4}\right) p_{2} \\
& =-4 p_{4} p_{3}^{2}-4 p_{4}^{2} p_{3}+8 p_{4} p_{3}-88 \sqrt{33}+504
\end{aligned}
$$

constrained by $\sum_{i=1}^{4} p_{i}=2$ and $0<p_{i}<2$, with others vanishing. 


\subsection{Polytope 8: $Y^{3,0}$}

The polytope is

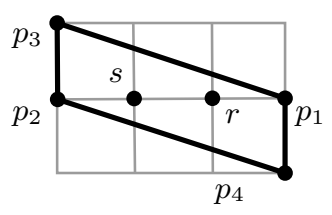

The brane tiling and the corresponding quiver are
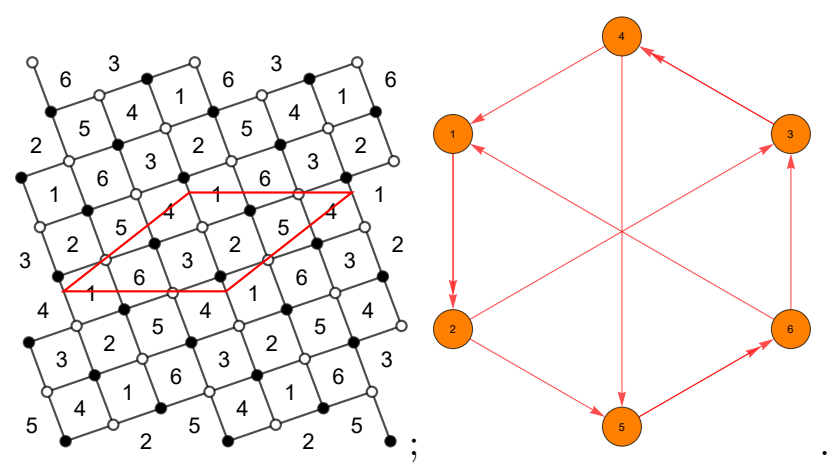

The superpotential is

$$
\begin{aligned}
W= & X_{41} X_{12}^{1} X_{23} X_{34}^{2}+X_{63} X_{34}^{1} X_{45} X_{56}^{2}+X_{25} X_{56}^{1} X_{61} X_{12}^{2} \\
& -X_{34}^{1} X_{41} X_{12}^{2} X_{23}-X_{56}^{1} X_{63} X_{34}^{2} X_{45}-X_{12}^{1} X_{25} X_{56}^{2} X_{61} .
\end{aligned}
$$

The perfect matching matrix is

$$
P=\left(\begin{array}{c|cccccccccccccccc} 
& r_{1} & s_{1} & s_{2} & r_{2} & p_{1} & p_{2} & s_{3} & r_{3} & s_{4} & p_{3} & s_{5} & r_{4} & r_{5} & p_{4} & s_{6} & r_{6} \\
\hline X_{12}^{1} & 0 & 1 & 0 & 0 & 1 & 0 & 0 & 0 & 0 & 0 & 0 & 1 & 0 & 0 & 0 & 0 \\
X_{12}^{2} & 0 & 1 & 0 & 0 & 0 & 1 & 0 & 0 & 0 & 0 & 0 & 1 & 0 & 0 & 0 & 0 \\
X_{23} & 0 & 0 & 1 & 0 & 0 & 0 & 1 & 0 & 0 & 1 & 0 & 0 & 1 & 0 & 1 & 0 \\
X_{25} & 1 & 0 & 1 & 1 & 0 & 0 & 0 & 0 & 0 & 0 & 0 & 0 & 1 & 1 & 0 & 0 \\
X_{34}^{1} & 1 & 0 & 0 & 0 & 1 & 0 & 0 & 0 & 1 & 0 & 0 & 0 & 0 & 0 & 0 & 0 \\
X_{34}^{2} & 1 & 0 & 0 & 0 & 0 & 1 & 0 & 0 & 1 & 0 & 0 & 0 & 0 & 0 & 0 & 0 \\
X_{41} & 0 & 0 & 0 & 1 & 0 & 0 & 0 & 1 & 0 & 0 & 1 & 0 & 0 & 1 & 0 & 1 \\
X_{45} & 0 & 1 & 1 & 1 & 0 & 0 & 0 & 0 & 0 & 1 & 1 & 0 & 0 & 0 & 0 & 0 \\
X_{56}^{1} & 0 & 0 & 0 & 0 & 1 & 0 & 1 & 1 & 0 & 0 & 0 & 0 & 0 & 0 & 0 & 0 \\
X_{56}^{56} & 0 & 0 & 0 & 0 & 0 & 1 & 1 & 1 & 0 & 0 & 0 & 0 & 0 & 0 & 0 & 0 \\
X_{61} & 0 & 0 & 0 & 0 & 0 & 0 & 0 & 0 & 1 & 1 & 1 & 0 & 0 & 0 & 1 & 1 \\
X_{63} & 0 & 0 & 0 & 0 & 0 & 0 & 0 & 0 & 0 & 0 & 0 & 1 & 1 & 1 & 1 & 1
\end{array}\right),
$$

where the relations between bifundamentals and GLSM fields can be directly read off. Then we can get the total charge matrix:

$$
Q_{t}=\left(\begin{array}{cccccccccccccccc}
r_{1} & s_{1} & s_{2} & r_{2} & p_{1} & p_{2} & s_{3} & r_{3} & s_{4} & p_{3} & s_{5} & r_{4} & r_{5} & p_{4} & s_{6} & r_{6} \\
\hline 1 & 1 & 0 & -1 & 0 & 0 & 0 & 0 & -1 & 0 & 0 & -1 & 0 & 0 & 0 & 1 \\
1 & 1 & -1 & 0 & 0 & 0 & 0 & 0 & -1 & 0 & 0 & -1 & 0 & 0 & 1 & 0 \\
0 & 1 & 0 & -1 & 0 & 0 & 0 & 0 & 0 & 0 & 0 & -1 & 0 & 1 & 0 & 0 \\
0 & 1 & -1 & 0 & 0 & 0 & 0 & 0 & 0 & 0 & 0 & -1 & 1 & 0 & 0 & 0 \\
1 & 0 & 0 & -1 & 0 & 0 & 0 & 0 & -1 & 0 & 1 & 0 & 0 & 0 & 0 & 0 \\
1 & 0 & -1 & 0 & 0 & 0 & 0 & 0 & -1 & 1 & 0 & 0 & 0 & 0 & 0 & 0 \\
1 & 1 & 0 & -1 & -1 & -1 & 0 & 1 & 0 & 0 & 0 & 0 & 0 & 0 & 0 & 0 \\
1 & 1 & -1 & 0 & -1 & -1 & 1 & 0 & 0 & 0 & 0 & 0 & 0 & 0 & 0 & 0 \\
\hline 1 & 1 & 0 & -1 & 1 & 1 & 0 & 0 & -2 & 0 & 0 & -1 & 0 & 0 & 0 & 0 \\
2 & 2 & 0 & -1 & -1 & -1 & 0 & 0 & -1 & 0 & 0 & 0 & 0 & 0 & 0 & 0 \\
2 & 1 & 0 & -2 & 0 & 0 & 0 & 0 & -1 & 0 & 0 & 0 & 0 & 0 & 0 & 0 \\
0 & 0 & 1 & -1 & 0 & 0 & 0 & 0 & -1 & 0 & 0 & 1 & 0 & 0 & 0 & 0 \\
1 & 2 & -1 & -1 & 0 & 0 & 0 & 0 & -1 & 0 & 0 & 0 & 0 & 0 & 0 & 0
\end{array}\right)
$$


with kernel

$$
G_{t}=\left(\begin{array}{cccccccccccccccc}
r_{1} & s_{1} & s_{2} & r_{2} & p_{1} & p_{2} & s_{3} & r_{3} & s_{4} & p_{3} & s_{5} & r_{4} & r_{5} & p_{4} & s_{6} & r_{6} \\
\hline 1 & 0 & 0 & 1 & 1 & 0 & 0 & 1 & 0 & -1 & 0 & 1 & 1 & 2 & 0 & 1 \\
0 & 1 & 1 & 0 & 1 & 0 & 1 & 0 & 1 & 2 & 1 & 0 & 0 & -1 & 1 & 0 \\
1 & 1 & 1 & 1 & 1 & 1 & 1 & 1 & 1 & 1 & 1 & 1 & 1 & 1 & 1 & 1
\end{array}\right) .
$$

From $G_{t}$, we can get the GLSM fields associated to each point as shown in (4.21), where

$$
r=\left\{r_{1}, \ldots, r_{6}\right\}, s=\left\{s_{1}, \ldots, s_{6}\right\} .
$$

From $Q_{t}$ (and $Q_{F}$ ), the mesonic symmetry reads $\mathrm{SU}(2) \times \mathrm{U}(1) \times \mathrm{U}(1)_{\mathrm{R}}$ and the baryonic symmetry reads $\mathrm{U}(1)_{\mathrm{h}}^{4} \times \mathrm{U}(1)$, where the subscripts "R" and " $\mathrm{h}$ " indicate $\mathrm{R}$ - and hidden symmetries respectively.

The Hilbert series of the toric cone is

$$
\begin{aligned}
H S= & \frac{1}{\left(1-t_{2}\right)\left(1-t_{1} t_{2}\right)\left(1-\frac{t_{3}}{t_{1} t_{2}^{2}}\right)}+\frac{1}{\left(1-\frac{1}{t_{2}}\right)\left(1-t_{1} t_{2}^{2}\right)\left(1-\frac{t_{3}}{t_{1} t_{2}}\right)} \\
& +\frac{1}{\left(1-t_{2}\right)\left(1-\frac{t_{1} t_{2}^{2}}{t_{3}}\right)\left(1-\frac{t_{3}^{2}}{t_{1} t_{2}^{3}}\right)}+\frac{1}{\left(1-\frac{1}{t_{2}}\right)\left(1-\frac{1}{t_{1} t_{2}^{2}}\right)\left(1-t_{1} t_{2}^{3} t_{3}\right)} \\
& +\frac{1}{\left(1-\frac{1}{t_{2}}\right)\left(1-\frac{t_{3}^{2}}{t_{1}}\right)\left(1-\frac{t_{1} t_{2}}{t_{3}}\right)}+\frac{1}{\left(1-t_{2}\right)\left(1-\frac{1}{t_{1} t_{2}}\right)\left(1-t_{1} t_{3}\right)} .
\end{aligned}
$$

The volume function is then

$$
V=\frac{81}{\left(b_{1}-6\right)\left(b_{1}+3\right)\left(b_{1}+3 b_{2}-6\right)\left(b_{1}+3 b_{2}+3\right)} .
$$

Minimizing $V$ yields $V_{\min }=16 / 81$ at $b_{1}=3 / 2, b_{2}=0$. Thus, $a_{\max }=81 / 64$. Together with the superconformal conditions, we can solve for the R-charges of the bifundamentals, which are $X_{I}=1 / 2$ for any $I$, viz, for all the bifundamentals. Hence, the R-charges of GLSM fields are $p_{i}=1 / 2$ with others vanishing.

\subsection{Polytope 9: $\mathrm{SPP} /\left(\mathbb{Z}_{2} \times \mathbb{Z}_{2}\right)(1,0,0,1)(0,1,1,0)$}

The polytope is

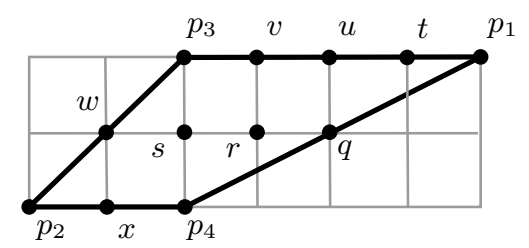

The brane tiling and the corresponding quiver are
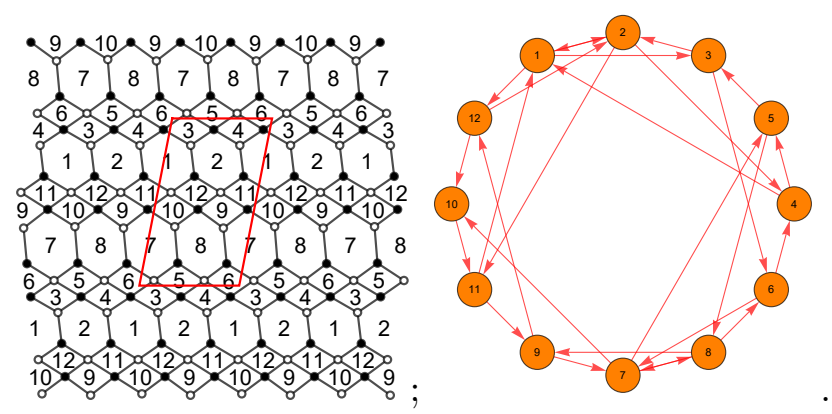
The superpotential is

$$
\begin{aligned}
W= & X_{1,3} X_{3,2} X_{2,1}+X_{2,4} X_{4,1} X_{1,2}+X_{6,7} X_{7,5} X_{5,3} X_{3,6}+X_{5,8} X_{8,6} X_{6,4} X_{4,5} \\
& +X_{8,9} X_{9,7} X_{7,8}+X_{7,10} X_{10,8} X_{8,7}+X_{12,2} X_{2,11} X_{11,9} X_{9,12}+X_{10,11} X_{11,1} X_{1,12} X_{12,10} \\
& -X_{2,4} X_{4,5} X_{5,3} X_{3,2}-X_{1,3} X_{3,6} X_{6,4} X_{4,1}-X_{7,5} X_{5,8} X_{8,7}-X_{8,6} X_{6,7} X_{7,8} \\
& -X_{7,10} X_{10,11} X_{11,9} X_{9,7}-X_{8,9} X_{9,12} X_{12,10} X_{10,8}-X_{2,11} X_{11,1} X_{1,2}-X_{1,12} X_{12,2} X_{2,1}
\end{aligned}
$$

The number of perfect matchings is $c=84$, which leads to gigantic $P, Q_{t}$ and $G_{t}$. Hence, we will not list them here. The GLSM fields associated to each point are shown in (4.30), where

$$
\begin{aligned}
& q=\left\{q_{1}, q_{2}\right\}, \quad r=\left\{r_{1}, \ldots, r_{30}\right\}, \quad s=\left\{s_{1}, \ldots, s_{30}\right\}, \quad t=\left\{t_{1}, \ldots, t_{4}\right\}, \\
& u=\left\{u_{1}, \ldots, u_{6}\right\}, \quad v=\left\{v_{1}, \ldots, v_{4}\right\}, \quad w=\left\{w_{1}, w_{2}\right\}, \quad x=\left\{x_{1}, x_{2}\right\} .
\end{aligned}
$$

The mesonic symmetry reads $\mathrm{U}(1)^{2} \times \mathrm{U}(1)_{\mathrm{R}}$ and the baryonic symmetry reads $\mathrm{U}(1)_{\mathrm{h}}^{4} \times \mathrm{U}(1)^{7}$, where the subscripts "R" and "h" indicate R- and hidden symmetries respectively.

The Hilbert series of the toric cone is

$$
\begin{aligned}
H S= & \frac{1}{\left(1-t_{2}\right)\left(1-\frac{t_{1} t_{2}}{t_{3}}\right)\left(1-\frac{t_{3}^{2}}{t_{1} t_{2}^{2}}\right)}+\frac{1}{\left(1-t_{2} t_{3}\right)\left(1-\frac{t_{1} t_{2}}{t_{3}^{2}}\right)\left(1-\frac{t_{3}^{2}}{t_{1} t_{2}^{2}}\right)} \\
& +\frac{1}{\left(1-\frac{1}{t_{2}}\right)\left(1-\frac{t_{3}^{2}}{t_{1}}\right)\left(1-\frac{t_{1} t_{2}}{t_{3}}\right)}+\frac{1}{\left(1-\frac{t_{1}}{t_{3}^{2}}\right)\left(1-t_{2} t_{3}\right)\left(1-\frac{t_{3}^{2}}{t_{1} t_{2}}\right)} \\
& +\frac{1}{\left(1-t_{2}\right)\left(1-\frac{1}{t_{1} t_{2}}\right)\left(1-t_{1} t_{3}\right)}+\frac{1}{\left(1-\frac{1}{t_{1}}\right)\left(1-t_{1} t_{2}\right)\left(1-\frac{t_{3}}{t_{2}}\right)} \\
& +\frac{1}{\left(1-t_{1}\right)\left(1-t_{2}\right)\left(1-\frac{t_{3}}{t_{1} t_{2}}\right)}+\frac{1}{\left(1-t_{1}\right)\left(1-\frac{1}{t_{1} t_{2}}\right)\left(1-t_{2} t_{3}\right)} \\
& +\frac{1}{\left(1-\frac{t_{1}}{t_{3}}\right)\left(1-t_{2} t_{3}\right)\left(1-\frac{t_{3}}{t_{1} t_{2}}\right)}+\frac{1}{\left(1-\frac{1}{t_{1}}\right)\left(1-\frac{1}{t_{2}}\right)\left(1-t_{1} t_{2} t_{3}\right)} \\
& +\frac{1}{\left(1-\frac{1}{t_{1} t_{3}}\right)\left(1-\frac{t_{3}}{t_{2}}\right)\left(1-t_{1} t_{2} t_{3}\right)}+\frac{1}{\left(1-\frac{1}{t_{2}}\right)\left(1-t_{1} t_{2}\right)\left(1-\frac{t_{3}}{t_{1}}\right)} .
\end{aligned}
$$

The volume function is then

$$
V=-\frac{2\left(b_{2}-9\right)}{\left(b_{2}-3\right)\left(b_{2}+3\right)\left(b_{1}+b_{2}+3\right)\left(b_{1}+2 b_{2}-6\right)} .
$$

Minimizing $V$ yields $V_{\min }=\sqrt{3} / 18$ at $b_{1}=3 \sqrt{3}-3, b_{2}=3-2 \sqrt{3}$. Thus, $a_{\max }=$ $3 \sqrt{3} / 2$. Together with the superconformal conditions, we can solve for the R-charges of the bifundamentals. Then the R-charges of GLSM fields are

$$
p_{1}=0.557091, p_{2}=p_{3}=0.5, p_{4}=0.442909
$$

with others vanishing. 
4.5 Polytope 10: $L^{2,3,2} / \mathbb{Z}_{2}(1,0,0,1)$

The polytope is

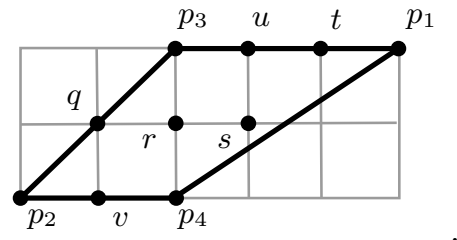

The brane tiling and the corresponding quiver are
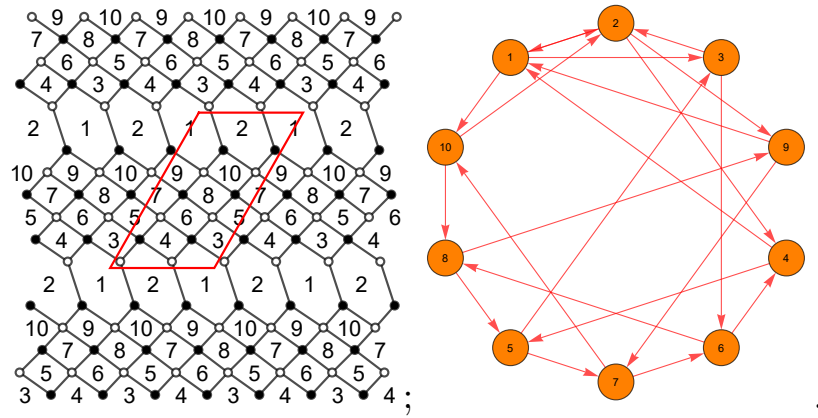

The superpotential is

$$
\begin{aligned}
W= & X_{13} X_{32} X_{21}+X_{24} X_{41} X_{12}+X_{68} X_{85} X_{53} X_{36} \\
& +X_{57} X_{76} X_{64} X_{45}+X_{10,2} X_{29} X_{97} X_{7,10}+X_{91} X_{1,10} X_{10,8} X_{89} \\
& -X_{13} X_{36} X_{64} X_{41}-X_{24} X_{45} X_{53} X_{32}-X_{57} X_{7,10} X_{10,8} X_{85} \\
& -X_{68} X_{89} X_{97} X_{76}-X_{29} X_{91} X_{12}-X_{1,10} X_{10,2} X_{21} .
\end{aligned}
$$


The perfect matching matrix is

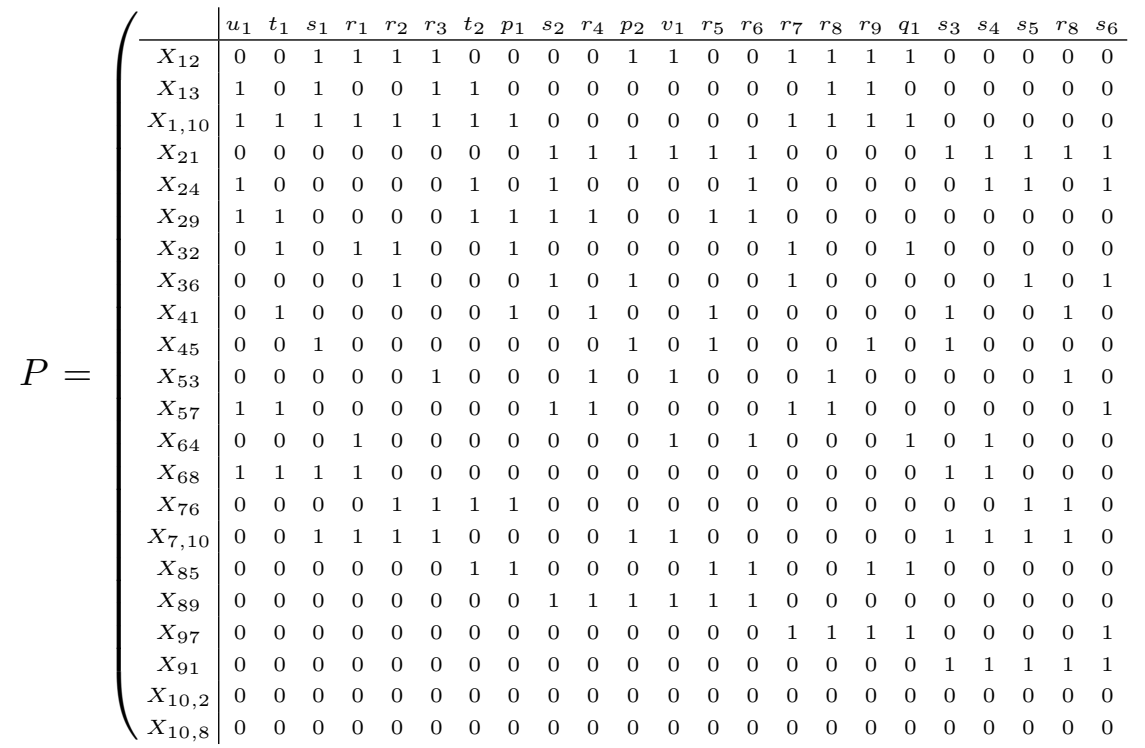

$\left.\begin{array}{cccccccccccccccccccccc}r_{10} & r_{11} & r_{12} & s_{7} & s_{8} & s_{9} & r_{13} & p_{3} & u_{2} & u_{3} & t_{3} & r_{14} & r_{15} & r_{16} & q_{2} & v_{2} & p_{4} & s_{10} & r_{17} & r_{18} & r_{19} \\ \hline 0 & 0 & 0 & 1 & 1 & 1 & 1 & 0 & 0 & 0 & 0 & 0 & 0 & 0 & 0 & 1 & 1 & 1 & 1 & 1 & 1 \\ 0 & 0 & 0 & 0 & 1 & 1 & 0 & 1 & 0 & 1 & 0 & 0 & 0 & 0 & 0 & 0 & 0 & 1 & 0 & 0 & 1 \\ 0 & 0 & 0 & 0 & 0 & 0 & 0 & 0 & 0 & 0 & 0 & 0 & 0 & 0 & 0 & 0 & 0 & 0 & 0 & 0 & 0 \\ 1 & 1 & 1 & 0 & 0 & 0 & 0 & 0 & 0 & 0 & 0 & 1 & 1 & 1 & 1 & 1 & 1 & 0 & 0 & 0 & 0 \\ 0 & 0 & 1 & 0 & 0 & 0 & 0 & 1 & 0 & 1 & 0 & 0 & 1 & 1 & 0 & 0 & 0 & 0 & 0 & 0 & 0 \\ 0 & 0 & 0 & 0 & 0 & 0 & 0 & 0 & 0 & 0 & 0 & 1 & 1 & 1 & 1 & 0 & 0 & 0 & 0 & 0 & 0 \\ 0 & 0 & 0 & 1 & 0 & 0 & 1 & 0 & 1 & 0 & 1 & 0 & 0 & 0 & 0 & 0 & 0 & 0 & 1 & 1 & 0 \\ 0 & 0 & 0 & 1 & 0 & 0 & 0 & 0 & 0 & 0 & 0 & 0 & 0 & 1 & 0 & 1 & 0 & 0 & 0 & 1 & 0 \\ 1 & 1 & 0 & 0 & 0 & 0 & 0 & 0 & 1 & 0 & 1 & 1 & 0 & 0 & 1 & 0 & 0 & 0 & 0 & 0 & 0 \\ 0 & 1 & 0 & 0 & 0 & 1 & 0 & 0 & 0 & 0 & 0 & 1 & 0 & 0 & 0 & 1 & 0 & 1 & 0 & 0 & 0 \\ 1 & 0 & 0 & 0 & 1 & 0 & 0 & 0 & 0 & 0 & 0 & 0 & 0 & 0 & 1 & 0 & 1 & 0 & 0 & 0 & 1 \\ 1 & 0 & 0 & 1 & 1 & 0 & 0 & 1 & 1 & 0 & 0 & 0 & 0 & 0 & 0 & 0 & 0 & 0 & 0 & 0 & 0 \\ 0 & 0 & 1 & 0 & 0 & 0 & 1 & 0 & 0 & 0 & 0 & 0 & 1 & 0 & 0 & 0 & 1 & 0 & 1 & 0 & 0 \\ 0 & 0 & 0 & 0 & 0 & 0 & 0 & 1 & 1 & 0 & 0 & 1 & 1 & 0 & 0 & 0 & 0 & 1 & 1 & 0 & 0 \\ 0 & 0 & 0 & 0 & 0 & 0 & 0 & 0 & 0 & 1 & 1 & 0 & 0 & 1 & 1 & 0 & 0 & 0 & 0 & 1 & 1 \\ 0 & 0 & 0 & 0 & 0 & 0 & 0 & 0 & 0 & 0 & 0 & 0 & 0 & 0 & 0 & 0 & 0 & 0 & 0 & 0 & 0 \\ 0 & 1 & 1 & 0 & 0 & 1 & 1 & 0 & 0 & 1 & 1 & 0 & 0 & 0 & 0 & 0 & 0 & 0 & 0 & 0 & 0 \\ 0 & 0 & 0 & 1 & 1 & 1 & 1 & 0 & 0 & 0 & 0 & 0 & 0 & 0 & 0 & 0 & 0 & 0 & 0 & 0 & 0 \\ 1 & 1 & 1 & 0 & 0 & 0 & 0 & 0 & 0 & 0 & 0 & 0 & 0 & 0 & 0 & 1 & 1 & 0 & 0 & 0 & 0 \\ 1 & 1 & 1 & 0 & 0 & 0 & 0 & 1 & 1 & 1 & 1 & 0 & 0 & 0 & 0 & 0 & 0 & 0 & 0 & 0 & 0 \\ 0 & 0 & 0 & 1 & 1 & 1 & 1 & 1 & 1 & 1 & 1 & 0 & 0 & 0 & 0 & 0 & 0 & 1 & 1 & 1 & 1 \\ 0 & 0 & 0 & 0 & 0 & 0 & 0 & 0 & 0 & 0 & 0 & 1 & 1 & 1 & 1 & 1 & 1 & 1 & 1 & 1 & 1\end{array}\right)$,


where the relations between bifundamentals and GLSM fields can be directly read off. Then we can get the total charge matrix:

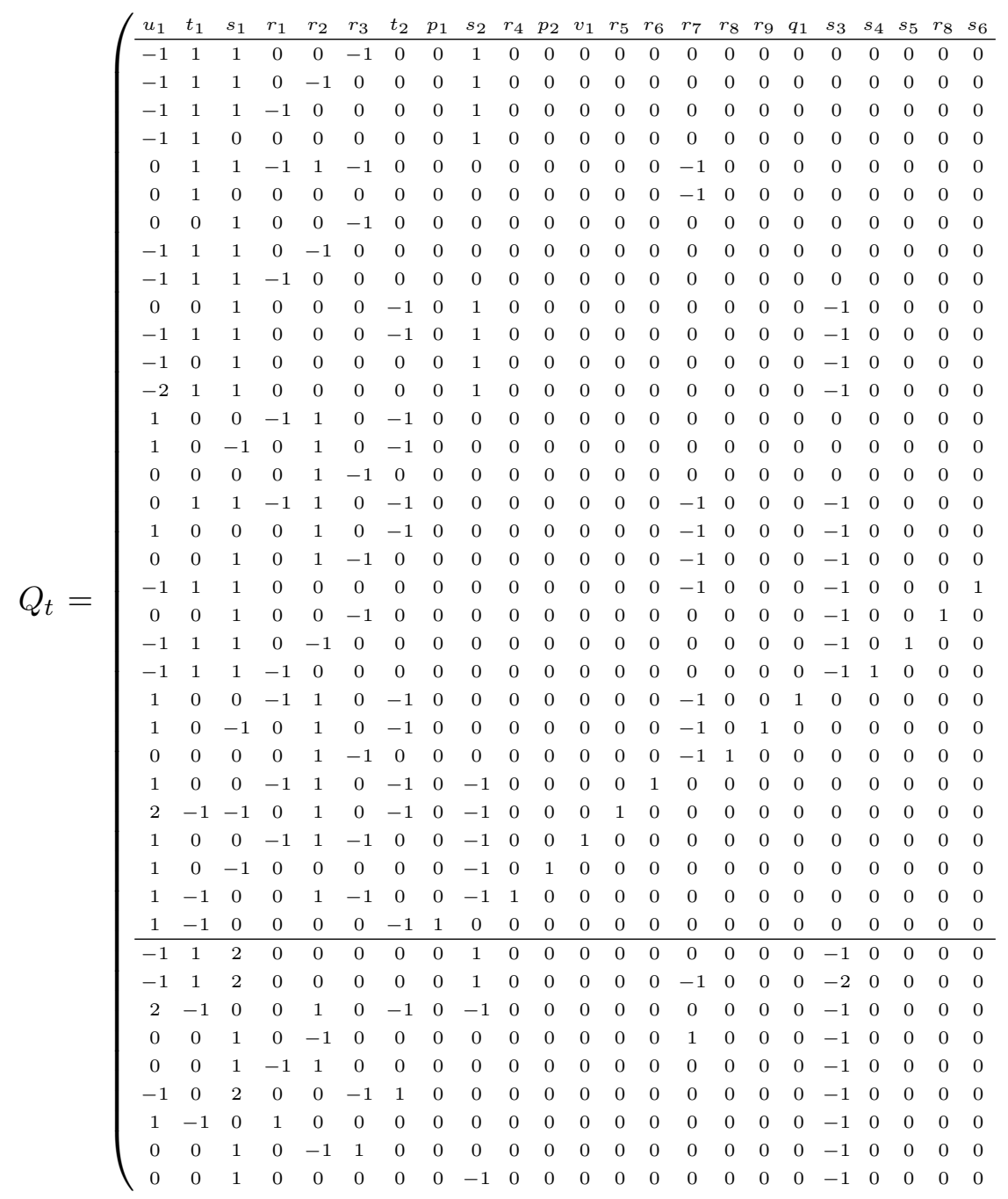




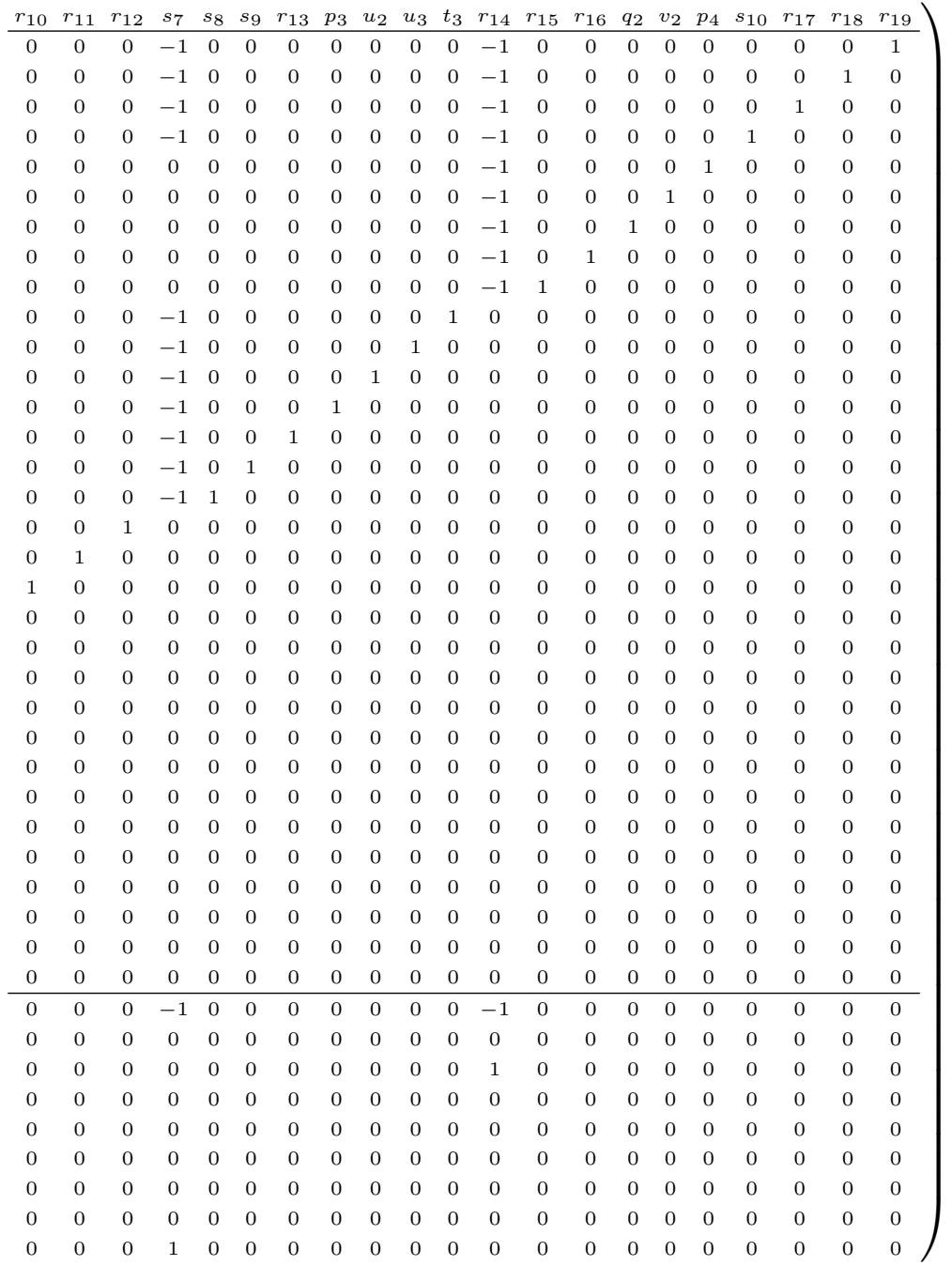

with kernel

$$
\begin{aligned}
& G_{t}=\left(\begin{array}{cccccccccccccccccccccccc}
u_{1} & t_{1} & s_{1} & r_{1} & r_{2} & r_{3} & t_{2} & p_{1} & s_{2} & r_{4} & p_{2} & v_{1} & r_{5} & r_{6} & r_{7} & r_{8} & r_{9} & q_{1} & s_{3} & s_{4} & s_{5} & r_{8} & s_{6} \\
\hline 2 & 3 & 0 & 1 & 1 & 1 & 3 & 4 & 0 & 1 & -2 & -1 & 1 & 1 & 1 & 1 & 1 & 2 & 0 & 0 & 0 & 1 & 0 \\
0 & -1 & 1 & 0 & 0 & 0 & -1 & -2 & 1 & 0 & 2 & 1 & 0 & 0 & 0 & 0 & 0 & -1 & 1 & 1 & 1 & 0 & 1 \\
1 & 1 & 1 & 1 & 1 & 1 & 1 & 1 & 1 & 1 & 1 & 1 & 1 & 1 & 1 & 1 & 1 & 1 & 1 & 1 & 1 & 1 & 1
\end{array}\right. \\
& \left.\begin{array}{ccccccccccccccccccccc}
r_{10} & r_{11} & r_{12} & s_{7} & s_{8} & s_{9} & r_{13} & p_{3} & u_{2} & u_{3} & t_{3} & r_{14} & r_{15} & r_{16} & q_{2} & v_{2} & p_{4} & s_{10} & r_{17} & r_{18} & r_{19} \\
\hline 1 & 1 & 1 & 0 & 0 & 0 & 1 & 1 & 2 & 2 & 3 & 1 & 1 & 1 & 2 & -1 & 0 & 0 & 1 & 1 & 1 \\
0 & 0 & 0 & 1 & 1 & 1 & 0 & 1 & 0 & 0 & -1 & 0 & 0 & 0 & -1 & 1 & 0 & 1 & 0 & 0 & 0 \\
1 & 1 & 1 & 1 & 1 & 1 & 1 & 1 & 1 & 1 & 1 & 1 & 1 & 1 & 1 & 1 & 1 & 1 & 1 & 1 & 1
\end{array}\right) .
\end{aligned}
$$

From $G_{t}$, we can get the GLSM fields associated to each point as shown in (4.37), where

$$
\begin{aligned}
& q=\left\{q_{1}, q_{2}\right\}, \quad r=\left\{r_{1}, \ldots, r_{19}\right\}, \quad t=\left\{t_{1}, \ldots, t_{3}\right\}, \\
& v=\left\{v_{1}, v_{2}\right\}, \quad s=\left\{s_{1}, \ldots, s_{10}\right\}, \quad u=\left\{u_{1}, \ldots, u_{3}\right\} .
\end{aligned}
$$

From $Q_{t}$ (and $Q_{F}$ ), the mesonic symmetry reads $\mathrm{U}(1)^{2} \times \mathrm{U}(1)_{\mathrm{R}}$ and the baryonic symmetry reads $\mathrm{U}(1)_{\mathrm{h}}^{4} \times \mathrm{U}(1)^{5}$, where the subscripts " $\mathrm{R}$ " and "h" indicate $\mathrm{R}$ - and hidden symmetries respectively. 
The Hilbert series of the toric cone is

$$
\begin{aligned}
H S= & \frac{1}{\left(1-\frac{t_{1} t_{2}}{t_{3}}\right)\left(1-\frac{t_{1} t_{2}^{2}}{t_{3}}\right)\left(1-\frac{t_{3}^{3}}{t_{1}^{2} t_{2}^{3}}\right)}+\frac{1}{\left(1-t_{2} t_{3}\right)\left(1-\frac{t_{1} t_{2}}{t_{3}}\right)\left(1-\frac{t_{3}}{t_{1} t_{2}^{2}}\right)} \\
& +\frac{1}{\left(1-t_{2}\right)\left(1-\frac{1}{t_{1} t_{2}}\right)\left(1-t_{1} t_{3}\right)}+\frac{1}{\left(1-\frac{1}{t_{1}}\right)\left(1-t_{1} t_{2}\right)\left(1-\frac{t_{3}}{t_{2}}\right)} \\
& +\frac{1}{\left(1-t_{1}\right)\left(1-t_{2}\right)\left(1-\frac{t_{3}}{t_{1} t_{2}}\right)}+\frac{1}{\left(1-t_{1}\right)\left(1-\frac{1}{t_{1} t_{2}}\right)\left(1-t_{2} t_{3}\right)} \\
& +\frac{1}{\left(1-\frac{t_{1}}{t_{3}}\right)\left(1-t_{2} t_{3}\right)\left(1-\frac{t_{3}}{t_{1} t_{2}}\right)}+\frac{1}{\left(1-\frac{1}{t_{1}}\right)\left(1-\frac{1}{t_{2}}\right)\left(1-t_{1} t_{2} t_{3}\right)} \\
& +\frac{1}{\left(1-\frac{1}{t_{1} t_{3}}\right)\left(1-\frac{t_{3}}{t_{2}}\right)\left(1-t_{1} t_{2} t_{3}\right)}+\frac{1}{\left(1-\frac{1}{t_{2}}\right)\left(1-t_{1} t_{2}\right)\left(1-\frac{t_{3}}{t_{1}}\right)} .
\end{aligned}
$$

The volume function is then

$$
V=-\frac{2\left(b_{2}-15\right)}{\left(b_{2}-3\right)\left(b_{2}+3\right)\left(b_{1}+b_{2}+3\right)\left(2 b_{1}+3 b_{2}-9\right)} .
$$

Minimizing $V$ yields $V_{\min }=(10+7 \sqrt{7}) / 243$ at $b_{1}=(5 \sqrt{7}-11) / 2, b_{2}=5+2 \sqrt{7}$. Thus, $a_{\max }=(-10+7 \sqrt{7}) / 4$. Together with the superconformal conditions, we can solve for the R-charges of the bifundamentals. Then the R-charges of GLSM fields should satisfy

$$
\begin{gathered}
\left(81 p_{2}+81 p_{4}\right) p_{3}^{2}+\left(81 p_{2}^{2}+162 p_{4} p_{2}-162 p_{2}+81 p_{4}^{2}-162 p_{4}\right) p_{3} \\
=-54 p_{4} p_{2}^{2}-54 p_{4}^{2} p_{2}+108 p_{4} p_{2}-28 \sqrt{7}+40
\end{gathered}
$$

constrained by $\sum_{i=1}^{4} p_{i}=2$ and $0<p_{i}<2$, with others vanishing.

\subsection{Polytope 11: $\mathrm{dP}_{1} / \mathbb{Z}_{2}(1,0,0,1)$}

The polytope is

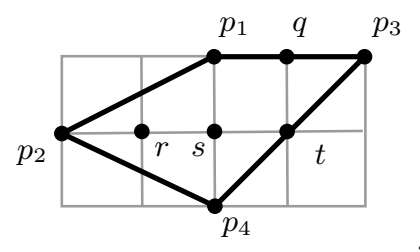

The brane tiling and the corresponding quiver are
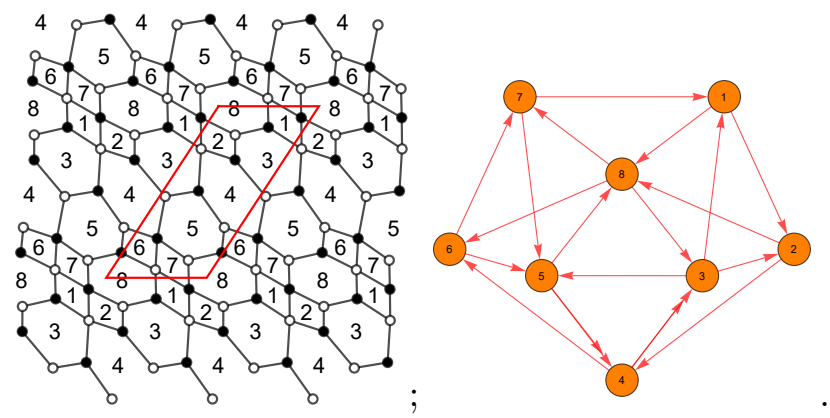
The superpotential is

$$
\begin{aligned}
W= & X_{83} X_{32} X_{28}+X_{12} X_{24} X_{43}^{1} X_{31}+X_{35} X_{54}^{1} X_{43}^{2} \\
& +X_{46} X_{65} X_{54}^{2}+X_{58} X_{87} X_{75}+X_{67} X_{71} X_{18} X_{86} \\
& -X_{18} X_{83} X_{31}-X_{32} X_{24} X_{43}^{2}-X_{43}^{1} X_{35} X_{54}^{2} \\
& -X_{65} X_{58} X_{86}-X_{54}^{1} X_{46} X_{67} X_{75}-X_{87} X_{71} X_{12} X_{28} .
\end{aligned}
$$

The perfect matching matrix is

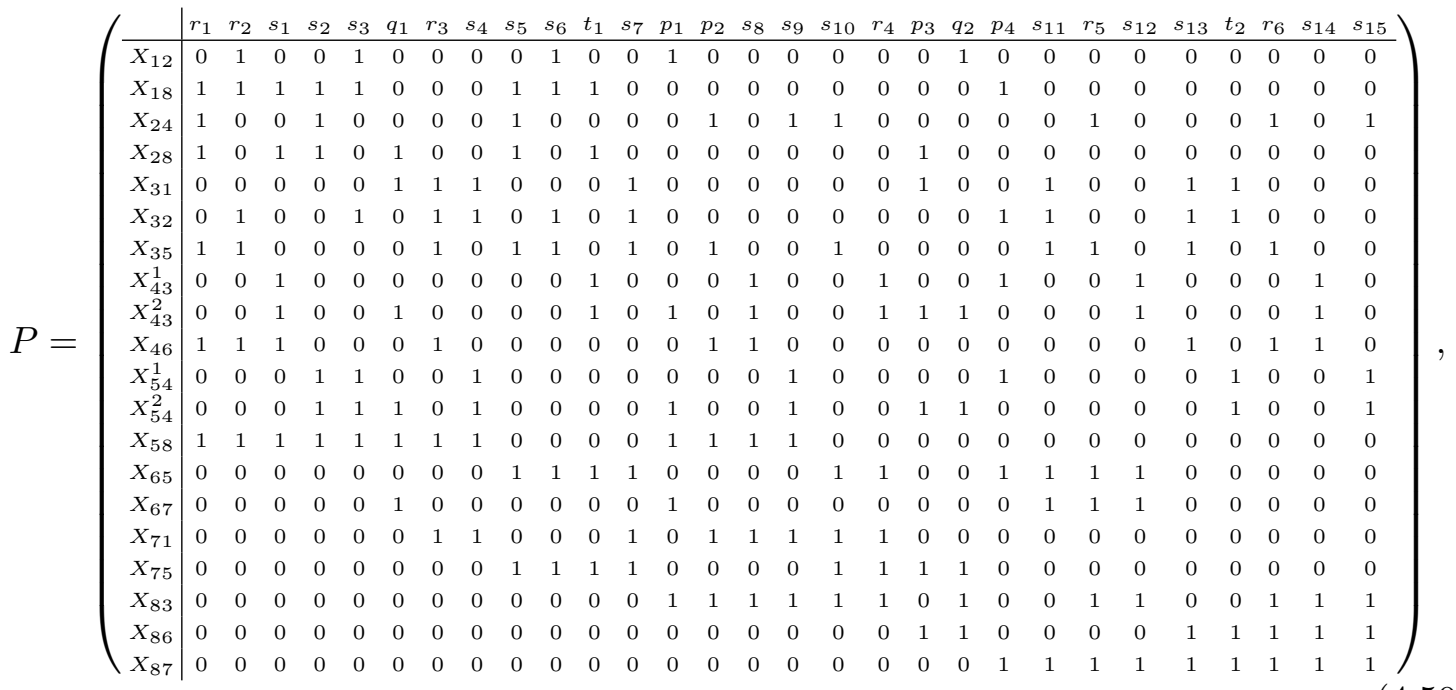

where the relations between bifundamentals and GLSM fields can be directly read off.

Then we can get the total charge matrix:

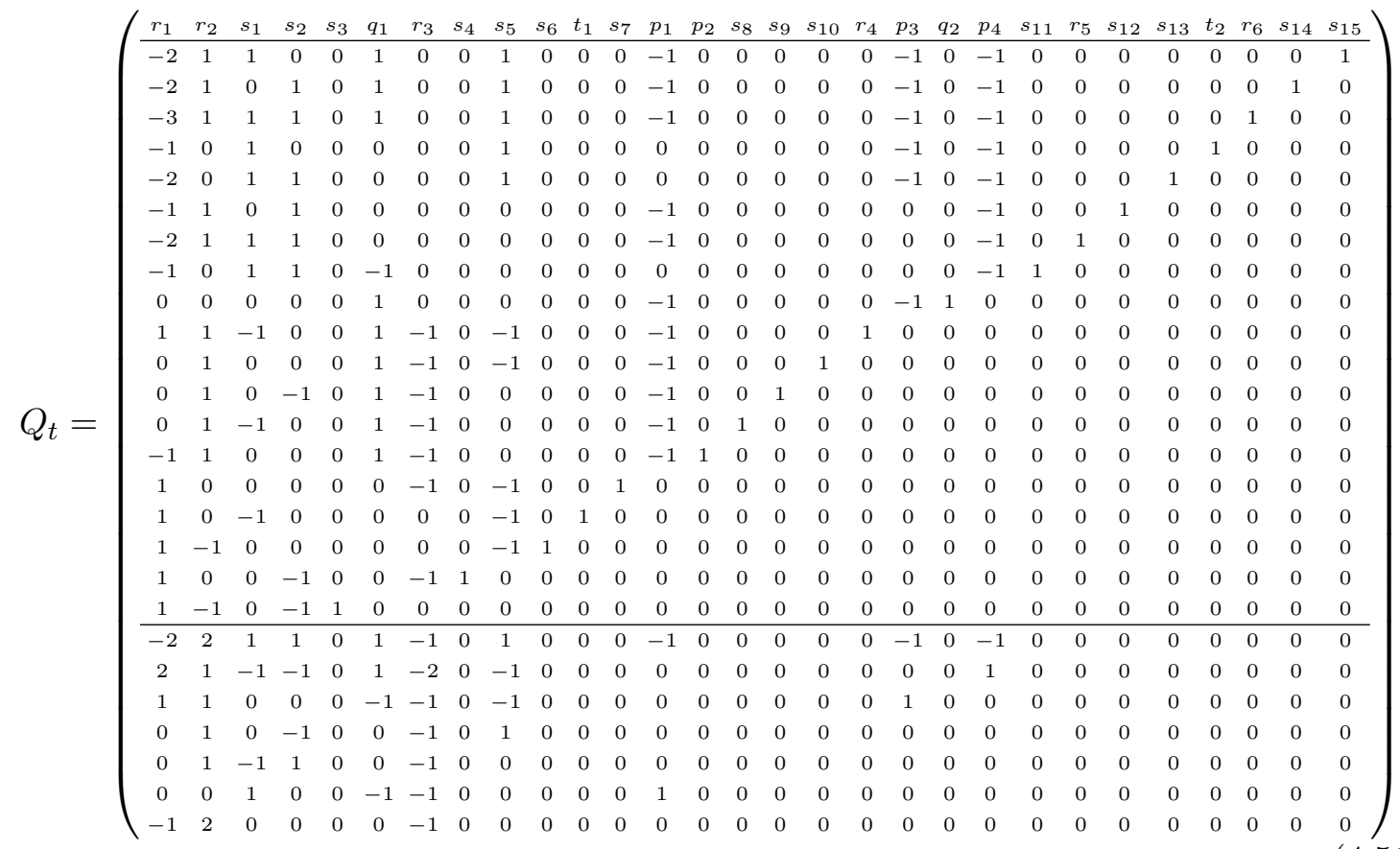


with kernel

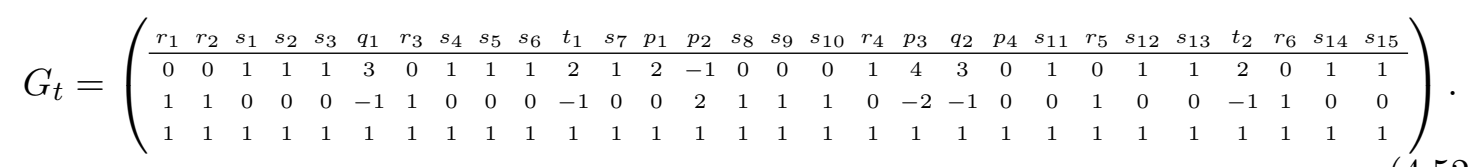

From $G_{t}$, we can get the GLSM fields associated to each point as shown in (4.47), where

$$
q=\left\{q_{1}, q_{2}\right\}, \quad r=\left\{r_{1}, \ldots, r_{5}\right\}, \quad s=\left\{s_{1}, \ldots, s_{15}\right\}, \quad t=\left\{t_{1}, t_{2}\right\} .
$$

From $Q_{t}$ (and $Q_{F}$ ), the mesonic symmetry reads $\mathrm{U}(1)^{2} \times \mathrm{U}(1)_{\mathrm{R}}$ and the baryonic symmetry reads $\mathrm{U}(1)_{\mathrm{h}}^{4} \times \mathrm{U}(1)^{3}$, where the subscripts " $\mathrm{R}$ " and " $\mathrm{h}$ " indicate $\mathrm{R}$ - and hidden symmetries respectively.

The Hilbert series of the toric cone is

$$
\begin{aligned}
H S= & \frac{1}{\left(1-t_{2}\right)\left(1-\frac{t_{1} t_{2}}{t_{3}}\right)\left(1-\frac{t_{3}^{2}}{t_{1} t_{2}^{2}}\right)}+\frac{1}{\left(1-\frac{1}{t_{2}}\right)\left(1-\frac{t_{1}}{t_{2} t_{3}}\right)\left(1-\frac{t_{2}^{2} t_{3}^{2}}{t_{1}}\right)} \\
& +\frac{1}{\left(1-\frac{1}{t_{1}}\right)\left(1-t_{1} t_{2}\right)\left(1-\frac{t_{3}}{t_{2}}\right)}+\frac{1}{\left(1-t_{1}\right)\left(1-t_{2}\right)\left(1-\frac{t_{3}}{t_{1} t_{2}}\right)} \\
& +\frac{1}{\left(1-t_{1}\right)\left(1-\frac{1}{t_{2}}\right)\left(1-\frac{t_{2} t_{3}}{t_{1}}\right)}+\frac{1}{\left(1-\frac{1}{t_{1}}\right)\left(1-\frac{1}{t_{2}}\right)\left(1-t_{1} t_{2} t_{3}\right)} \\
& +\frac{1}{\left(1-\frac{1}{t_{1} t_{3}}\right)\left(1-\frac{t_{3}}{t_{2}}\right)\left(1-t_{1} t_{2} t_{3}\right)}+\frac{1}{\left(1-t_{2}\right)\left(1-\frac{1}{t_{1} t_{2}}\right)\left(1-t_{1} t_{3}\right)} .
\end{aligned}
$$

The volume function is then

$$
V=\frac{2\left(b_{1}+4\left(b_{2}-6\right)\right)}{\left(b_{2}-3\right)\left(b_{1}+b_{2}+3\right)\left(b_{1}+2 b_{2}-6\right)\left(b_{1}-2\left(b_{2}+3\right)\right)} .
$$

Minimizing $V$ yields $V_{\min }=(46+13 \sqrt{13}) / 648$ at $b_{1}=0, b_{2}=4-\sqrt{13}$. Thus, $a_{\max }=$ $-92+26 \sqrt{13}$. Together with the superconformal conditions, we can solve for the R-charges of the bifundamentals. Then the R-charges of GLSM fields should satisfy

$$
\begin{gathered}
\left(108 p_{2}+177 p_{3}\right) p_{4}^{2}+\left(108 p_{2}^{2}+108 p_{3} p_{2}-216 p_{2}+177 p_{3}^{2}-354 p_{3}\right) p_{4} \\
=-54 p_{3} p_{2}^{2}-54 p_{3}^{2} p_{2}+108 p_{3} p_{2}-832 \sqrt{13}+2921
\end{gathered}
$$

constrained by $\sum_{i=1}^{4} p_{i}=2$ and $0<p_{i}<2$, with others vanishing.

\subsection{Polytope 12: $L^{1,4,1} / \mathbb{Z}_{2}(1,0,0,1)$}

The polytope is

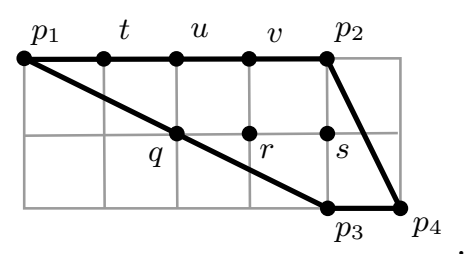


The brane tiling and the corresponding quiver are
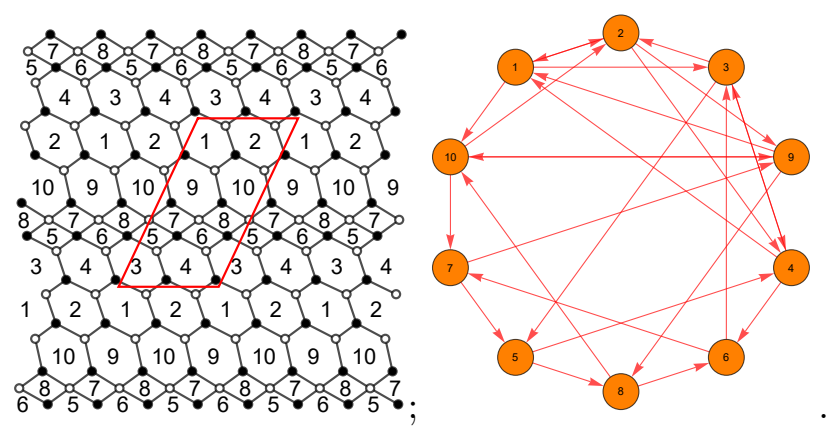

The superpotential is

$$
\begin{aligned}
W= & X_{13} X_{32} X_{21}+X_{24} X_{41} X_{12}+X_{35} X_{54} X_{43}+X_{46} X_{63} X_{34} \\
& +X_{58} X_{8,10} X_{10,7} X_{75}+X_{67} X_{79} X_{98} X_{86}+X_{10,2} X_{29} X_{9,10}+X_{91} X_{1,10} X_{10,9} \\
& -X_{41} X_{13} X_{34}-X_{32} X_{24} X_{43}-X_{63} X_{35} X_{58} X_{86}-X_{54} X_{46} X_{67} X_{75} \\
& -X_{10,7} X_{79} X_{9,10}-X_{98} X_{8,10} X_{10,9}-X_{29} X_{91} X_{12}-X_{1,10} X_{10,2} X_{21} .
\end{aligned}
$$

The number of perfect matchings is $c=50$, which leads to gigantic $P, Q_{t}$ and $G_{t}$. Hence, we will not list them here. The GLSM fields associated to each point are shown in (4.57), where

$$
\begin{array}{lll}
q=\left\{q_{1}, q_{2}\right\}, & r=\left\{r_{1}, \ldots, r_{20}\right\}, & s=\left\{s_{1}, \ldots, s_{10}\right\}, \\
t=\left\{t_{1}, \ldots, t_{4}\right\}, & u=\left\{u_{1}, \ldots, u_{6}\right\}, & v=\left\{v_{1}, \ldots, v_{4}\right\} .
\end{array}
$$

The mesonic symmetry reads $\mathrm{U}(1)^{2} \times \mathrm{U}(1)_{\mathrm{R}}$ and the baryonic symmetry reads $\mathrm{U}(1)_{\mathrm{h}}^{4} \times \mathrm{U}(1)^{5}$, where the subscripts "R" and "h" indicate R- and hidden symmetries respectively.

The Hilbert series of the toric cone is

$$
\begin{aligned}
H S= & \frac{1}{\left(1-\frac{1}{t_{1}}\right)\left(1-\frac{1}{t_{1} t_{2}}\right)\left(1-t_{1}^{2} t_{2} t_{3}\right)}+\frac{1}{\left(1-t_{2}\right)\left(1-\frac{t_{1} t_{2}}{t_{3}}\right)\left(1-\frac{t_{3}^{2}}{t_{1} t_{2}^{2}}\right)} \\
& +\frac{1}{\left(1-t_{2} t_{3}\right)\left(1-\frac{t_{1} t_{2}}{t_{3}}\right)\left(1-\frac{t_{3}}{t_{1} t_{2}^{2}}\right)}+\frac{1}{\left(1-\frac{1}{t_{2}}\right)\left(1-\frac{t_{1} t_{2}^{3}}{t_{3}}\right)\left(1-\frac{t_{3}^{2}}{t_{1} t_{2}^{2}}\right)} \\
& +\frac{1}{\left(1-t_{2} t_{3}\right)\left(1-\frac{t_{1} t_{2}^{2}}{t_{3}}\right)\left(1-\frac{t_{3}}{t_{1} t_{2}^{3}}\right)}+\frac{1}{\left(1-\frac{1}{t_{1}}\right)\left(1-t_{1} t_{2}\right)\left(1-\frac{t_{3}}{t_{2}}\right)} \\
& +\frac{1}{\left(1-t_{1}\right)\left(1-t_{2}\right)\left(1-\frac{t_{3}}{t_{1} t_{2}}\right)}+\frac{\left(1-t_{1}\right)\left(1-\frac{1}{t_{1} t_{2}}\right)\left(1-t_{2} t_{3}\right)}{1} \\
& +\frac{1}{\left(1-\frac{t_{1}}{t_{3}}\right)\left(1-t_{2} t_{3}\right)\left(1-\frac{t_{3}}{t_{1} t_{2}}\right)}+\frac{1}{\left(1-\frac{1}{t_{2}}\right)\left(1-t_{1} t_{2}\right)\left(1-\frac{t_{3}}{t_{1}}\right)} .
\end{aligned}
$$

The volume function is then

$$
V=-\frac{6\left(b_{2}-5\right)}{\left(b_{2}-3\right)\left(b_{2}+3\right)\left(2 b_{1}+b_{2}+3\right)\left(b_{1}+2 b_{2}-6\right)} .
$$


Minimizing $V$ yields $V_{\min }=(13 \sqrt{13}-35) / 108$ at $b_{1}=(5 \sqrt{13}+1) / 6, b_{2}=(5-2 \sqrt{13}) / 3$. Thus, $a_{\max }=(13 \sqrt{13}+35) / 36$. Together with the superconformal conditions, we can solve for the R-charges of the bifundamentals. Then the R-charges of GLSM fields should satisfy

$$
\begin{gathered}
\left(972 p_{2}+243 p_{4}\right) p_{3}^{2}+\left(972 p_{2}^{2}+1944 p_{4} p_{2}-1944 p_{2}+243 p_{4}^{2}-486 p_{4}\right) p_{3} \\
=-972 p_{4} p_{2}^{2}-972 p_{4}^{2} p_{2}+1944 p_{4} p_{2}-52 \sqrt{13}-140
\end{gathered}
$$

constrained by $\sum_{i=1}^{4} p_{i}=2$ and $0<p_{i}<2$, with others vanishing.

\subsection{Polytope 13: $\mathrm{PdP}_{2} / \mathbb{Z}_{2}(1,1,1,1)$}

The polytope is

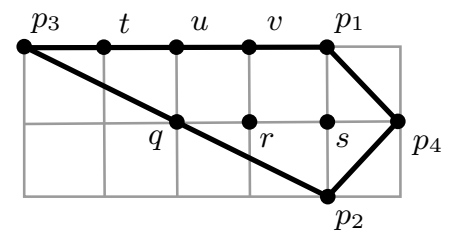

The brane tiling and the corresponding quiver are
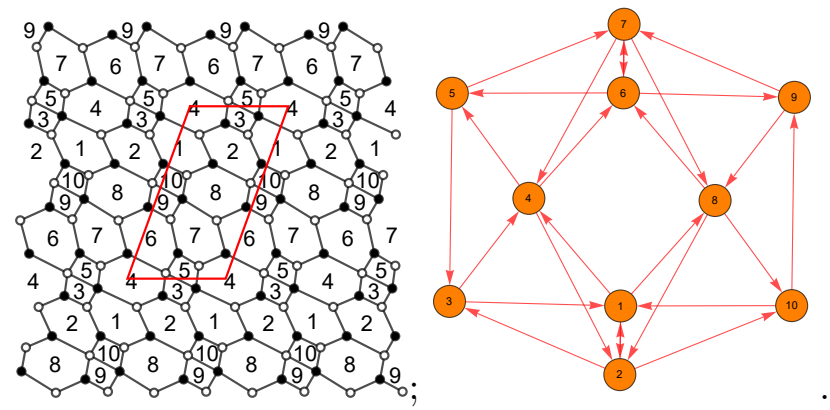

The superpotential is

$$
\begin{aligned}
W= & X_{14} X_{42} X_{21}+X_{23} X_{31} X_{12}+X_{46} X_{65} X_{53} X_{34}+X_{57} X_{74} X_{45} \\
& +X_{69} X_{97} X_{76}+X_{78} X_{86} X_{67}+X_{82} X_{2,10} X_{10,9} X_{98}+X_{10,1} X_{18} X_{8,10} \\
& -X_{31} X_{14} X_{45} X_{53}-X_{23} X_{34} X_{42}-X_{46} X_{67} X_{74}-X_{65} X_{57} X_{76} \\
& -X_{69} X_{98} X_{86}-X_{97} X_{78} X_{8,10} X_{10,9}-X_{2,10} X_{10,1} X_{12}-X_{18} X_{82} X_{21} .
\end{aligned}
$$

The number of perfect matchings is $c=53$, which leads to gigantic $P, Q_{t}$ and $G_{t}$. Hence, we will not list them here. The GLSM fields associated to each point are shown in (4.64), where

$$
\begin{aligned}
& q=\left\{q_{1}, q_{2}\right\}, \quad r=\left\{r_{1}, \ldots, r_{21}\right\}, \quad s=\left\{s_{1}, \ldots, s_{12}\right\}, \\
& t=\left\{t_{1}, \ldots, t_{4}\right\}, \quad u=\left\{u_{1}, \ldots, u_{6}\right\}, \quad v=\left\{v_{1}, \ldots, v_{4}\right\} .
\end{aligned}
$$

The mesonic symmetry reads $\mathrm{U}(1)^{2} \times \mathrm{U}(1)_{\mathrm{R}}$ and the baryonic symmetry reads $\mathrm{U}(1)_{\mathrm{h}}^{4} \times \mathrm{U}(1)^{5}$, where the subscripts "R" and "h" indicate R- and hidden symmetries respectively. 
The Hilbert series of the toric cone is

$$
\begin{aligned}
H S= & \frac{1}{\left(1-t_{2}\right)\left(1-\frac{t_{1} t_{2}}{t_{3}}\right)\left(1-\frac{t_{3}^{2}}{t_{1} t_{2}^{2}}\right)}+\frac{1}{\left(1-t_{2} t_{3}\right)\left(1-\frac{t_{1} t_{2}}{t_{3}^{2}}\right)\left(1-\frac{t_{3}^{2}}{t_{1} t_{2}^{2}}\right)} \\
& +\frac{1}{\left(1-\frac{t_{3}^{2}}{t_{1}}\right)\left(1-t_{2} t_{3}\right)\left(1-\frac{t_{1}}{t_{2} t_{3}^{2}}\right)}+\frac{1}{\left(1-\frac{t_{1}}{t_{3}^{2}}\right)\left(1-t_{2} t_{3}\right)\left(1-\frac{t_{3}^{2}}{t_{1} t_{2}}\right)} \\
& +\frac{1}{\left(1-\frac{1}{t_{2}}\right)\left(1-\frac{t_{1}}{t_{3}}\right)\left(1-\frac{t_{2} t_{3}^{2}}{t_{1}}\right)}+\frac{1}{\left(1-\frac{1}{t_{1}}\right)\left(1-t_{2}\right)\left(1-\frac{t_{1} t_{3}}{t_{2}}\right)} \\
& +\frac{1}{\left(1-\frac{t_{3}}{t_{1}}\right)\left(1-t_{2} t_{3}\right)\left(1-\frac{t_{1}}{t_{2} t_{3}}\right)}+\frac{1}{\left(1-t_{1}\right)\left(1-\frac{1}{t_{2}}\right)\left(1-\frac{t_{2} t_{3}}{t_{1}}\right)} \\
& +\frac{1}{\left(1-\frac{1}{t_{1}}\right)\left(1-\frac{1}{t_{2}}\right)\left(1-t_{1} t_{2} t_{3}\right)}+\frac{1}{\left(1-t_{1}\right)\left(1-t_{2}\right)\left(1-\frac{t_{3}}{t_{1} t_{2}}\right)}
\end{aligned}
$$

The volume function is then

$$
V=\frac{2\left(2 b_{1}+b_{2}+15\right)}{\left(b_{2}+3\right)\left(-b_{1}+b_{2}-3\right)\left(b_{1}+b_{2}+3\right)\left(b_{1}+2 b_{2}-6\right)} .
$$

Minimizing $V$ yields $V_{\min }=0.112571$ at $b_{1}=3.27464, b_{2}=-0.831239$. Thus, $a_{\max }=$ 2.220821. Together with the superconformal conditions, we can solve for the R-charges of the bifundamentals. Then the R-charges of GLSM fields should satisfy ${ }^{14}$

$$
\begin{gathered}
\left(6.75 p_{3}+1.6875 p_{4}\right) p_{2}^{2}+\left(6.75 p_{3}^{2}+6.75 p_{4} p_{3}-13.5 p_{3}+1.6875 p_{4}^{2}-3.375 p_{4}\right) p_{2} \\
=-3.375 p_{4} p_{3}^{2}-3.375 p_{4}^{2} p_{3}+6.75 p_{4} p_{3}-2.22082
\end{gathered}
$$

constrained by $\sum_{i=1}^{4} p_{i}=2$ and $0<p_{i}<2$, with others vanishing.

\subsection{Polytope 14: $L^{1,3,1} / \mathbb{Z}_{2}(1,0,0,1)$}

The polytope is

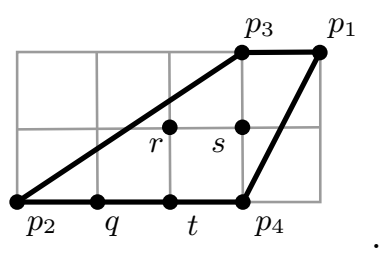

\footnotetext{
${ }^{14}$ For these Sasaki-Einstein manifolds that are not (quasi-)regular, the minimized volumes, and hence the following calculations, are solved numerically. However, we can actually use roots of some polynomials to express the exact results. The case in this subsection is given as an example in appendix B.
} 
The brane tiling and the corresponding quiver are

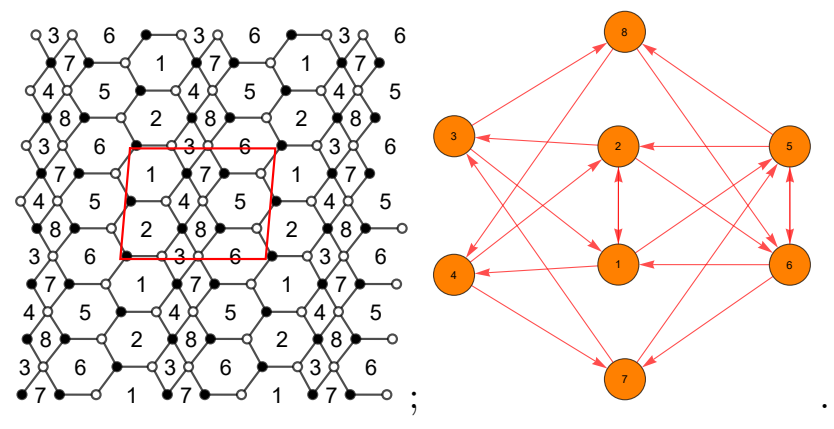

The superpotential is

$$
\begin{aligned}
W= & X_{61} X_{15} X_{56}+X_{52} X_{26} X_{65}+X_{23} X_{31} X_{12} \\
& +X_{14} X_{42} X_{21}+X_{38} X_{86} X_{67} X_{73}+X_{47} X_{75} X_{58} X_{84} \\
& -X_{15} X_{52} X_{21}-X_{26} X_{61} X_{12}-X_{23} X_{38} X_{84} X_{42} \\
& -X_{14} X_{47} X_{73} X_{31}-X_{75} X_{56} X_{67}-X_{86} X_{65} X_{58} .
\end{aligned}
$$

The perfect matching matrix is

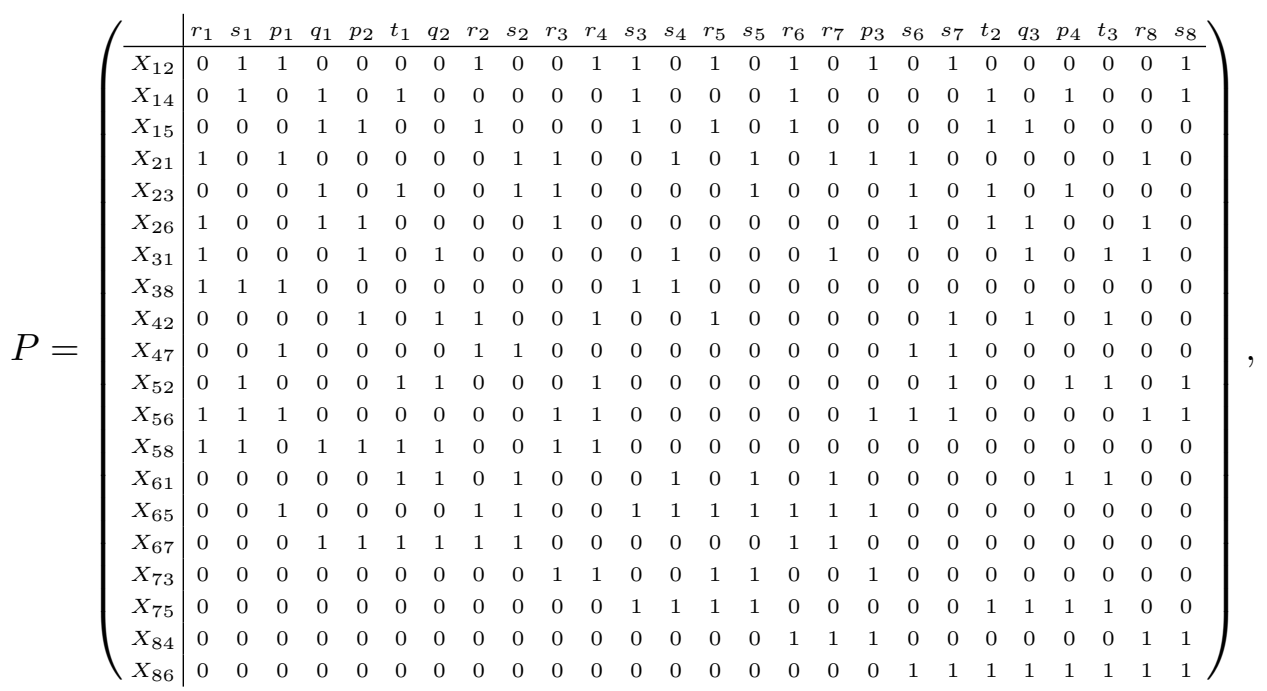


where the relations between bifundamentals and GLSM fields can be directly read off. Then we can get the total charge matrix:

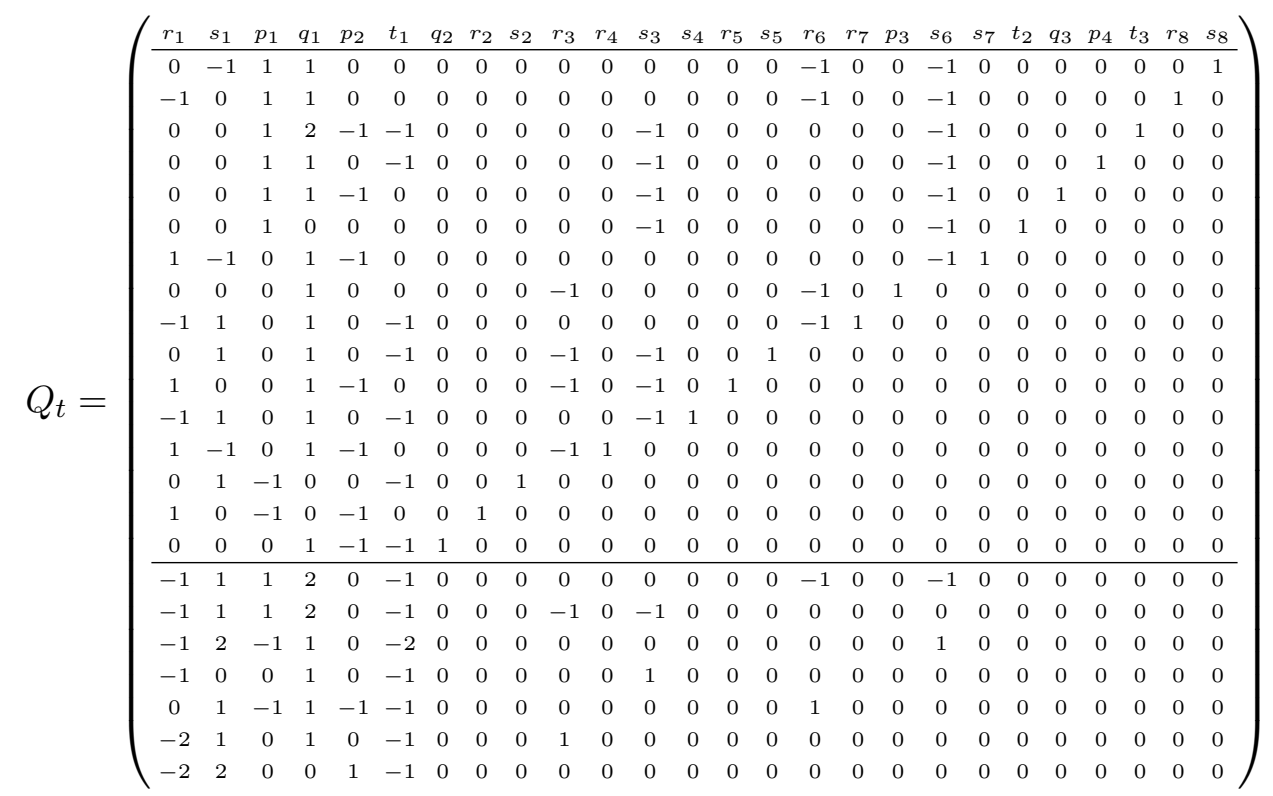

with kernel

$$
G_{t}=\left(\begin{array}{cccccccccccccccccccccccccc}
r_{1} & s_{1} & p_{1} & q_{1} & p_{2} & t_{1} & q_{2} & r_{2} & s_{2} & r_{3} & r_{4} & s_{3} & s_{4} & r_{5} & s_{5} & r_{6} & r_{7} & p_{3} & s_{6} & s_{7} & t_{2} & q_{3} & p_{4} & t_{3} & r_{8} & s_{8} \\
\hline 0 & 1 & 2 & -1 & -2 & 0 & -1 & 0 & 1 & 0 & 0 & 1 & 1 & 0 & 1 & 0 & 0 & 1 & 1 & 1 & 0 & -1 & 1 & 0 & 0 & 1 \\
1 & 0 & 0 & 1 & 2 & 0 & 1 & 1 & 0 & 1 & 1 & 0 & 0 & 1 & 0 & 1 & 1 & 1 & 0 & 0 & 0 & 1 & -1 & 0 & 1 & 0 \\
1 & 1 & 1 & 1 & 1 & 1 & 1 & 1 & 1 & 1 & 1 & 1 & 1 & 1 & 1 & 1 & 1 & 1 & 1 & 1 & 1 & 1 & 1 & 1 & 1 & 1
\end{array}\right) .
$$

From $G_{t}$, we can get the GLSM fields associated to each point as shown in (4.71), where

$$
q=\left\{q_{1}, \ldots, q_{3}\right\}, \quad r=\left\{r_{1}, \ldots, r_{8}\right\}, \quad s=\left\{s_{1}, \ldots, s_{8}\right\}, \quad t=\left\{t_{1}, \ldots, t_{3}\right\} .
$$

From $Q_{t}$ (and $\left.Q_{F}\right)$, the mesonic symmetry reads $\mathrm{U}(1)^{2} \times \mathrm{U}(1)_{\mathrm{R}}$ and the baryonic symmetry reads $\mathrm{U}(1)_{\mathrm{h}}^{4} \times \mathrm{U}(1)^{3}$, where the subscripts " $\mathrm{R}$ " and "h" indicate $\mathrm{R}$ - and hidden symmetries respectively.

The Hilbert series of the toric cone is

$$
\begin{aligned}
H S= & \frac{1}{\left(1-\frac{t_{1} t_{2}}{t_{3}}\right)\left(1-\frac{t_{1} t_{2}^{2}}{t_{3}}\right)\left(1-\frac{t_{3}^{3}}{t_{1}^{2} t_{2}^{3}}\right)}+\frac{1}{\left(1-\frac{1}{t_{1}}\right)\left(1-\frac{1}{t_{1} t_{2}}\right)\left(1-t_{1}^{2} t_{2} t_{3}\right)} \\
& +\frac{1}{\left(1-t_{2} t_{3}\right)\left(1-\frac{t_{1} t_{2}}{t_{3}}\right)\left(1-\frac{t_{3}}{t_{1} t_{2}^{2}}\right)}+\frac{1}{\left(1-t_{1}\right)\left(1-t_{2}\right)\left(1-\frac{t_{3}}{t_{1} t_{2}}\right)} \\
& +\frac{1}{\left(1-\frac{t_{3}}{t_{1}}\right)\left(1-t_{2} t_{3}\right)\left(1-\frac{t_{1}}{t_{2} t_{3}}\right)}+\frac{1}{\left(1-\frac{t_{1}}{t_{3}}\right)\left(1-t_{2} t_{3}\right)\left(1-\frac{t_{3}}{t_{1} t_{2}}\right)} \\
& +\frac{1}{\left(1-t_{1}\right)\left(1-\frac{1}{t_{2}}\right)\left(1-\frac{t_{2} t_{3}}{t_{1}}\right)}+\frac{1}{\left(1-\frac{1}{t_{1}}\right)\left(1-t_{1} t_{2}\right)\left(1-\frac{t_{3}}{t_{2}}\right)} .
\end{aligned}
$$

The volume function is then

$$
V=-\frac{8\left(b_{2}-6\right)}{\left(b_{2}-3\right)\left(b_{2}+3\right)\left(2 b_{1}+b_{2}+3\right)\left(2 b_{1}+3 b_{2}-9\right)} .
$$


Minimizing $V$ yields $V_{\min }=\frac{4}{243}(-10+7 \sqrt{7})$ at $b_{1}=(2 \sqrt{7}-1) / 2, b_{2}=2-\sqrt{7}$. Thus, $a_{\max }=(10+7 \sqrt{7}) / 16$. Together with the superconformal conditions, we can solve for the R-charges of the bifundamentals. Then the R-charges of GLSM fields should satisfy

$$
\begin{gathered}
\left(27 p_{2}+27 p_{4}\right) p_{3}^{2}+\left(27 p_{2}^{2}+54 p_{4} p_{2}-54 p_{2}+27 p_{4}^{2}-54 p_{4}\right) p_{3} \\
=-81 p_{4} p_{2}^{2}-81 p_{4}^{2} p_{2}+162 p_{4} p_{2}-7 \sqrt{7}-10
\end{gathered}
$$

constrained by $\sum_{i=1}^{4} p_{i}=2$ and $0<p_{i}<2$, with others vanishing.

\subsection{Polytope 15: $L^{3,5,2}$}

The polytope is

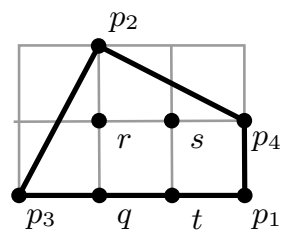

The brane tiling and the corresponding quiver are
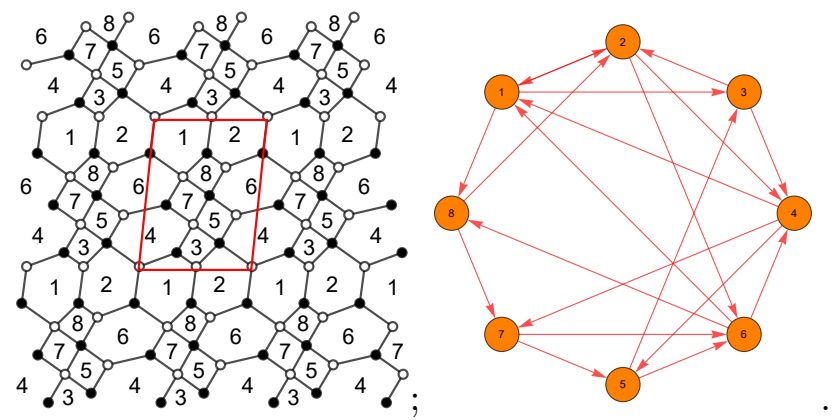

The superpotential is

$$
\begin{aligned}
W= & X_{24} X_{41} X_{12}+X_{13} X_{32} X_{21}+X_{47} X_{75} X_{53} X_{34} \\
& +X_{56} X_{64} X_{45}+X_{61} X_{18} X_{87} X_{76}+X_{82} X_{26} X_{68} \\
& -X_{13} X_{34} X_{41}-X_{24} X_{45} X_{53} X_{32}-X_{47} X_{76} X_{64} \\
& -X_{56} X_{68} X_{87} X_{75}-X_{26} X_{61} X_{12}-X_{18} X_{82} X_{21}
\end{aligned}
$$


The perfect matching matrix is

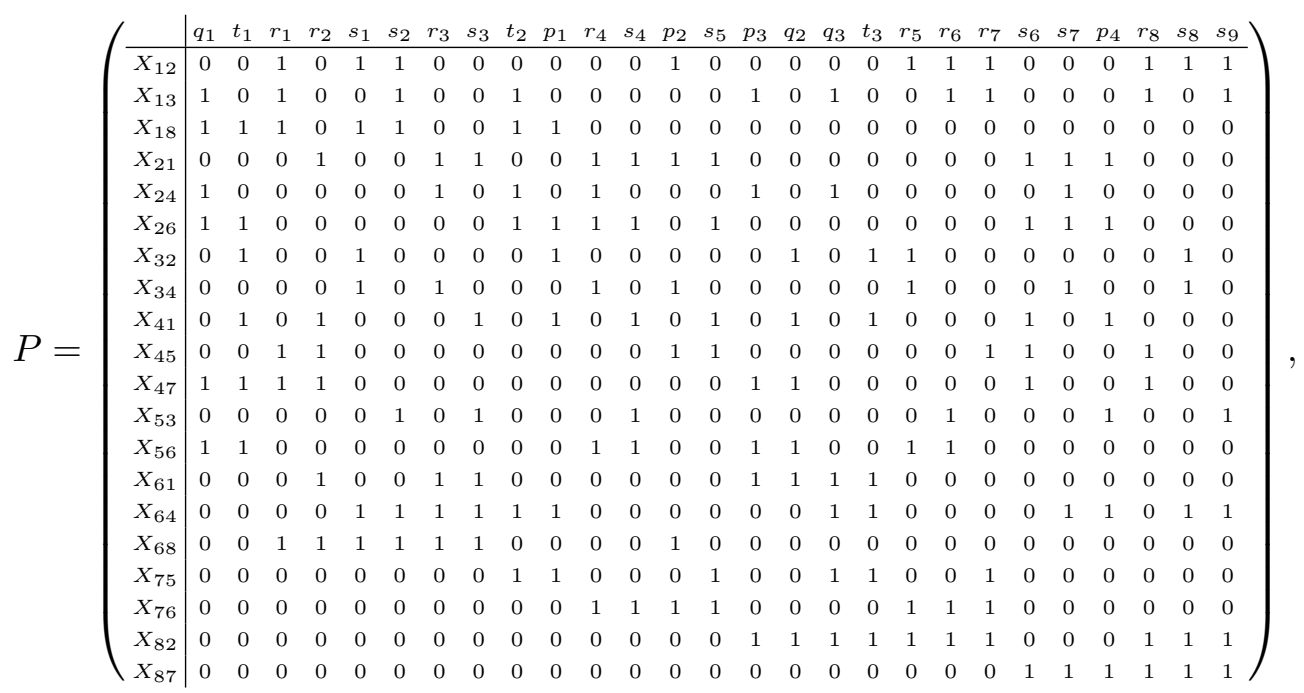

where the relations between bifundamentals and GLSM fields can be directly read off. Then we can get the total charge matrix:

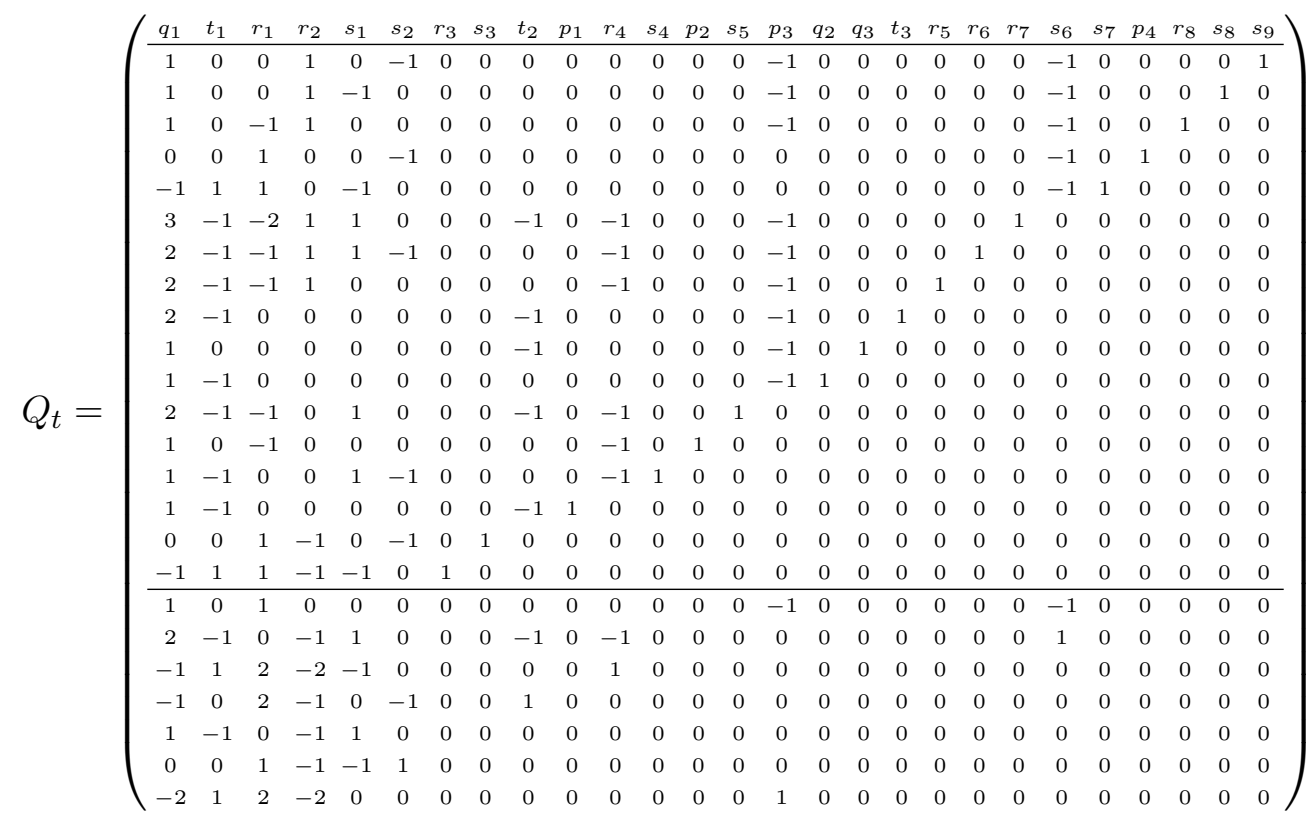

with kernel

$$
G_{t}=\left(\begin{array}{ccccccccccccccccccccccccccccccc}
q_{1} & t_{1} & r_{1} & r_{2} & s_{1} & s_{2} & r_{3} & s_{3} & t_{2} & p_{1} & r_{4} & s_{4} & p_{2} & s_{5} & p_{3} & q_{2} & q_{3} & t_{3} & r_{5} & r_{6} & r_{7} & s_{6} & s_{7} & p_{4} & r_{8} & s_{8} & s_{9} \\
\hline-1 & 0 & 0 & 0 & 1 & 1 & 0 & 1 & 0 & 1 & 0 & 1 & 1 & 1 & -2 & -1 & -1 & 0 & 0 & 0 & 0 & 1 & 1 & 2 & 0 & 1 & 1 \\
1 & 0 & 1 & 1 & 0 & 0 & 1 & 0 & 0 & -1 & 1 & 0 & 1 & 0 & 2 & 1 & 1 & 0 & 1 & 1 & 1 & 0 & 0 & -1 & 1 & 0 & 0 \\
1 & 1 & 1 & 1 & 1 & 1 & 1 & 1 & 1 & 1 & 1 & 1 & 1 & 1 & 1 & 1 & 1 & 1 & 1 & 1 & 1 & 1 & 1 & 1 & 1 & 1 & 1
\end{array}\right) .
$$

From $G_{t}$, we can get the GLSM fields associated to each point as shown in (4.81), where

$$
q=\left\{q_{1}, \ldots, q_{3}\right\}, \quad r=\left\{r_{1}, \ldots, r_{8}\right\}, \quad s=\left\{s_{1}, \ldots, s_{9}\right\}, \quad t=\left\{t_{1}, \ldots, t_{3}\right\} .
$$

From $Q_{t}$ (and $Q_{F}$ ), the mesonic symmetry reads $\mathrm{U}(1)^{2} \times \mathrm{U}(1)_{\mathrm{R}}$ and the baryonic symmetry reads $\mathrm{U}(1)_{\mathrm{h}}^{4} \times \mathrm{U}(1)^{3}$, where the subscripts " $\mathrm{R}$ " and " $\mathrm{h}$ " indicate $\mathrm{R}$ - and hidden symmetries respectively. 
The Hilbert series of the toric cone is

$$
\begin{aligned}
H S= & \frac{1}{\left(1-\frac{t_{1} t_{2}}{t_{3}}\right)\left(1-\frac{t_{1} t_{2}^{2}}{t_{3}}\right)\left(1-\frac{t_{3}^{3}}{t_{1}^{2} t_{2}^{3}}\right)}+\frac{1}{\left(1-t_{2} t_{3}\right)\left(1-\frac{t_{1} t_{2}}{t_{3}}\right)\left(1-\frac{t_{3}}{t_{1} t_{2}^{2}}\right)} \\
& +\frac{1}{\left(1-t_{1}\right)\left(1-t_{2}\right)\left(1-\frac{t_{3}}{t_{1} t_{2}}\right)}+\frac{1}{\left(1-\frac{1}{t_{1}}\right)\left(1-t_{2}\right)\left(1-\frac{t_{1} t_{3}}{t_{2}}\right)} \\
& +\frac{1}{\left(1-t_{1}\right)\left(1-\frac{1}{t_{1} t_{2}}\right)\left(1-t_{2} t_{3}\right)}+\frac{1}{\left(1-\frac{t_{1}}{t_{3}}\right)\left(1-t_{2} t_{3}\right)\left(1-\frac{t_{3}}{t_{1} t_{2}}\right)} \\
& +\frac{1}{\left(1-\frac{1}{t_{1}}\right)\left(1-\frac{1}{t_{2}}\right)\left(1-t_{1} t_{2} t_{3}\right)}+\frac{1}{\left(1-\frac{1}{t_{2}}\right)\left(1-t_{1} t_{2}\right)\left(1-\frac{t_{3}}{t_{1}}\right)} .
\end{aligned}
$$

The volume function is then

$$
V=\frac{2\left(3 b_{1}+2 b_{2}+24\right)}{\left(b_{2}+3\right)\left(-b_{1}+b_{2}-3\right)\left(b_{1}+b_{2}+3\right)\left(2 b_{1}+3 b_{2}-9\right)} .
$$

Minimizing $V$ yields $V_{\min }=0.142613$ at $b_{1}=2.194882, b_{2}=-0.760489$. Thus, $a_{\max }=$ 1.752996. Together with the superconformal conditions, we can solve for the R-charges of the bifundamentals. Then the R-charges of GLSM fields should satisfy

$$
\begin{aligned}
& \left(6.77917 p_{3}+2.25972 p_{4}\right) p_{2}^{2}+\left(6.77917 p_{3}^{2}+6.77917 p_{4} p_{3}-13.5583 p_{3}\right. \\
& \left.+2.25972 p_{4}^{2}-4.51945 p_{4}\right) p_{2}=-3.38958 p_{4} p_{3}^{2}-3.38958 p_{4}^{2} p_{3}+6.77917 p_{4} p_{3}-2.34743
\end{aligned}
$$

constrained by $\sum_{i=1}^{4} p_{i}=2$ and $0<p_{i}<2$, with others vanishing.

\subsection{Polytope $16 ; L^{2,5,1}$}

The polytope is

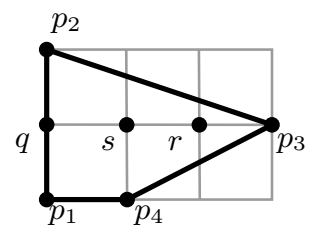

The brane tiling and the corresponding quiver are
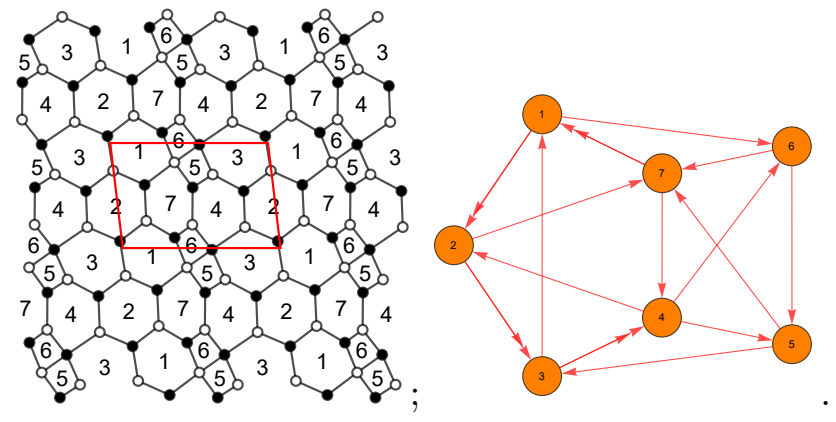
The superpotential is

$$
\begin{aligned}
W= & X_{31} X_{12}^{1} X_{23}^{2}+X_{27} X_{71}^{1} X_{12}^{2}+X_{71}^{2} X_{16} X_{65} X_{57} \\
& +X_{74} X_{46} X_{67}+X_{53} X_{34}^{1} X_{45}+X_{42} X_{23}^{1} X_{34}^{2} \\
& -X_{12}^{1} X_{27} X_{71}^{2}-X_{23}^{1} X_{31} X_{12}^{2}-X_{71}^{1} X_{16} X_{67} \\
& -X_{57} X_{74} X_{45}-X_{46} X_{65} X_{53} X_{34}^{2}-X_{34}^{1} X_{42} X_{23}^{2} .
\end{aligned}
$$

The perfect matching matrix is

$$
P=\left(\begin{array}{c|cccccccccccccccccccccccc} 
& s_{1} & s_{2} & r_{1} & q_{1} & s_{3} & s_{4} & s_{5} & r_{2} & s_{6} & s_{7} & r_{3} & p_{1} & p_{2} & q_{2} & s_{8} & s_{9} & r_{4} & r_{5} & r_{6} & p_{3} & p_{4} & s_{10} & s_{11} & r_{7} \\
\hline X_{12}^{1} & 0 & 1 & 0 & 1 & 0 & 0 & 1 & 0 & 0 & 1 & 0 & 1 & 0 & 0 & 0 & 1 & 0 & 0 & 1 & 0 & 1 & 0 & 0 & 0 \\
X_{12}^{2} & 0 & 1 & 0 & 1 & 0 & 0 & 1 & 0 & 0 & 1 & 0 & 0 & 1 & 0 & 0 & 1 & 0 & 0 & 1 & 0 & 0 & 0 & 0 & 0 \\
X_{16} & 1 & 1 & 1 & 0 & 0 & 0 & 0 & 0 & 0 & 0 & 0 & 0 & 0 & 0 & 0 & 1 & 1 & 1 & 1 & 1 & 0 & 0 & 0 & 0 \\
X_{23}^{1} & 1 & 0 & 0 & 0 & 0 & 1 & 0 & 0 & 1 & 0 & 0 & 1 & 0 & 1 & 0 & 0 & 0 & 1 & 0 & 0 & 1 & 0 & 1 & 0 \\
X_{23}^{2} & 1 & 0 & 0 & 0 & 0 & 1 & 0 & 0 & 1 & 0 & 0 & 0 & 1 & 1 & 0 & 0 & 0 & 1 & 0 & 0 & 0 & 0 & 1 & 0 \\
X_{27} & 1 & 0 & 1 & 0 & 1 & 1 & 0 & 1 & 1 & 0 & 1 & 0 & 0 & 0 & 0 & 0 & 1 & 1 & 0 & 1 & 0 & 0 & 0 & 0 \\
X_{31} & 0 & 0 & 1 & 0 & 1 & 0 & 0 & 1 & 0 & 0 & 1 & 0 & 0 & 0 & 1 & 0 & 1 & 0 & 0 & 1 & 0 & 1 & 0 & 1 \\
X_{34}^{1} & 0 & 0 & 0 & 1 & 1 & 0 & 0 & 0 & 0 & 0 & 0 & 1 & 0 & 0 & 0 & 1 & 1 & 0 & 0 & 0 & 1 & 1 & 0 & 0 \\
X_{34}^{2} & 0 & 0 & 0 & 1 & 1 & 0 & 0 & 0 & 0 & 0 & 0 & 0 & 1 & 0 & 0 & 1 & 1 & 0 & 0 & 0 & 0 & 1 & 0 & 0 \\
X_{42} & 0 & 1 & 1 & 0 & 0 & 0 & 1 & 1 & 0 & 1 & 1 & 0 & 0 & 0 & 1 & 0 & 0 & 0 & 1 & 1 & 0 & 0 & 0 & 1 \\
X_{45} & 1 & 1 & 1 & 0 & 0 & 0 & 0 & 0 & 1 & 1 & 1 & 0 & 1 & 1 & 1 & 0 & 0 & 0 & 0 & 0 & 0 & 0 & 0 & 0 \\
X_{46} & 1 & 1 & 1 & 0 & 0 & 0 & 0 & 0 & 0 & 0 & 0 & 1 & 0 & 1 & 1 & 0 & 0 & 0 & 0 & 0 & 0 & 0 & 0 & 0 \\
X_{53} & 0 & 0 & 0 & 0 & 0 & 1 & 1 & 1 & 0 & 0 & 0 & 0 & 0 & 0 & 0 & 0 & 0 & 1 & 1 & 1 & 0 & 0 & 1 & 1 \\
X_{57} & 0 & 0 & 0 & 1 & 1 & 1 & 1 & 1 & 0 & 0 & 0 & 1 & 0 & 0 & 0 & 0 & 0 & 0 & 0 & 0 & 0 & 0 & 0 & 0 \\
X_{65} & 0 & 0 & 0 & 0 & 0 & 0 & 0 & 0 & 1 & 1 & 1 & 0 & 0 & 0 & 0 & 0 & 0 & 0 & 0 & 0 & 1 & 0 & 0 & 0 \\
X_{67} & 0 & 0 & 0 & 1 & 1 & 1 & 1 & 1 & 1 & 1 & 1 & 0 & 1 & 0 & 0 & 0 & 0 & 0 & 0 & 0 & 0 & 0 & 0 & 0 \\
X_{71}^{1} & 0 & 0 & 0 & 0 & 0 & 0 & 0 & 0 & 0 & 0 & 0 & 1 & 0 & 1 & 1 & 0 & 0 & 0 & 0 & 0 & 1 & 1 & 1 & 1 \\
X_{71}^{2} & 0 & 0 & 0 & 0 & 0 & 0 & 0 & 0 & 0 & 0 & 0 & 0 & 1 & 1 & 1 & 0 & 0 & 0 & 0 & 0 & 0 & 1 & 1 & 1 \\
X_{74} & 0 & 0 & 0 & 0 & 0 & 0 & 0 & 0 & 0 & 0 & 0 & 0 & 0 & 0 & 0 & 1 & 1 & 1 & 1 & 1 & 1 & 1 & 1 & 1
\end{array}\right),
$$

where the relations between bifundamentals and GLSM fields can be directly read off. Then we can get the total charge matrix:

$$
Q_{t}=\left(\begin{array}{cccccccccccccccccccccccc}
s_{1} & s_{2} & r_{1} & q_{1} & s_{3} & s_{4} & s_{5} & r_{2} & s_{6} & s_{7} & r_{3} & p_{1} & p_{2} & q_{2} & s_{8} & s_{9} & r_{4} & r_{5} & r_{6} & p_{3} & p_{4} & s_{10} & s_{11} & r_{7} \\
\hline 2 & 0 & -1 & 2 & 0 & -1 & 0 & 0 & 0 & 0 & 0 & -1 & -1 & 0 & 0 & -1 & 0 & 0 & 0 & 0 & 0 & 0 & 0 & 1 \\
1 & 0 & 0 & 2 & 0 & -1 & 0 & 0 & 0 & 0 & 0 & -1 & -1 & 0 & 0 & -1 & 0 & 0 & 0 & 0 & 0 & 0 & 1 & 0 \\
1 & 1 & -1 & 1 & 0 & 0 & 0 & 0 & 0 & 0 & 0 & -1 & -1 & 0 & 0 & -1 & 0 & 0 & 0 & 0 & 0 & 1 & 0 & 0 \\
1 & 0 & 0 & 1 & 0 & 0 & 0 & 0 & -1 & 0 & 0 & -1 & 0 & 0 & 0 & -1 & 0 & 0 & 0 & 0 & 1 & 0 & 0 & 0 \\
1 & 0 & -1 & 1 & 0 & -1 & 0 & 0 & 0 & 0 & 0 & 0 & 0 & 0 & 0 & -1 & 0 & 0 & 0 & 1 & 0 & 0 & 0 & 0 \\
1 & -1 & 0 & 1 & 0 & -1 & 0 & 0 & 0 & 0 & 0 & 0 & 0 & 0 & 0 & -1 & 0 & 0 & 1 & 0 & 0 & 0 & 0 & 0 \\
0 & 0 & 0 & 1 & 0 & -1 & 0 & 0 & 0 & 0 & 0 & 0 & 0 & 0 & 0 & -1 & 0 & 1 & 0 & 0 & 0 & 0 & 0 & 0 \\
0 & 1 & -1 & 0 & 0 & 0 & 0 & 0 & 0 & 0 & 0 & 0 & 0 & 0 & 0 & -1 & 1 & 0 & 0 & 0 & 0 & 0 & 0 & 0 \\
1 & 0 & -1 & 1 & 0 & 0 & 0 & 0 & 0 & 0 & 0 & -1 & -1 & 0 & 1 & 0 & 0 & 0 & 0 & 0 & 0 & 0 & 0 & 0 \\
0 & 0 & 0 & 1 & 0 & 0 & 0 & 0 & 0 & 0 & 0 & -1 & -1 & 1 & 0 & 0 & 0 & 0 & 0 & 0 & 0 & 0 & 0 & 0 \\
1 & 0 & -1 & 0 & 0 & 0 & 0 & 0 & -1 & 0 & 1 & 0 & 0 & 0 & 0 & 0 & 0 & 0 & 0 & 0 & 0 & 0 & 0 & 0 \\
1 & -1 & 0 & 0 & 0 & 0 & 0 & 0 & -1 & 1 & 0 & 0 & 0 & 0 & 0 & 0 & 0 & 0 & 0 & 0 & 0 & 0 & 0 & 0 \\
1 & 0 & -1 & 0 & 0 & -1 & 0 & 1 & 0 & 0 & 0 & 0 & 0 & 0 & 0 & 0 & 0 & 0 & 0 & 0 & 0 & 0 & 0 & 0 \\
1 & -1 & 0 & 0 & 0 & -1 & 1 & 0 & 0 & 0 & 0 & 0 & 0 & 0 & 0 & 0 & 0 & 0 & 0 & 0 & 0 & 0 & 0 & 0 \\
0 & 1 & -1 & -1 & 1 & 0 & 0 & 0 & 0 & 0 & 0 & 0 & 0 & 0 & 0 & 0 & 0 & 0 & 0 & 0 & 0 & 0 & 0 & 0 \\
\hline 2 & 1 & -1 & 3 & 0 & 0 & 0 & 0 & 0 & 0 & 0 & -2 & -2 & 0 & 0 & -1 & 0 & 0 & 0 & 0 & 0 & 0 & 0 & 0 \\
2 & 1 & -1 & 1 & 0 & 0 & 0 & 0 & -1 & 0 & 0 & -1 & -1 & 0 & 0 & 0 & 0 & 0 & 0 & 0 & 0 & 0 & 0 & 0 \\
1 & 1 & -1 & 1 & 0 & -1 & 0 & 0 & 1 & 0 & 0 & -1 & -1 & 0 & 0 & 0 & 0 & 0 & 0 & 0 & 0 & 0 & 0 & 0 \\
1 & 0 & -1 & 1 & 0 & 0 & 0 & 0 & 0 & 0 & 0 & -1 & -1 & 0 & 0 & 1 & 0 & 0 & 0 & 0 & 0 & 0 & 0 & 0 \\
1 & 2 & -2 & 0 & 0 & 1 & 0 & 0 & 0 & 0 & 0 & -1 & -1 & 0 & 0 & 0 & 0 & 0 & 0 & 0 & 0 & 0 & 0 & 0 \\
0 & 2 & -1 & 1 & 0 & 0 & 0 & 0 & 0 & 0 & 0 & -1 & -1 & 0 & 0 & 0 & 0 & 0 & 0 & 0 & 0 & 0 & 0 & 0
\end{array}\right)
$$

with kernel

$$
G_{t}=\left(\begin{array}{cccccccccccccccccccccccc}
s_{1} & s_{2} & r_{1} & q_{1} & s_{3} & s_{4} & s_{5} & r_{2} & s_{6} & s_{7} & r_{3} & p_{1} & p_{2} & q_{2} & s_{8} & s_{9} & r_{4} & r_{5} & r_{6} & p_{3} & p_{4} & s_{10} & s_{11} & r_{7} \\
\hline 0 & 0 & 1 & -1 & 0 & 0 & 0 & 1 & 0 & 0 & 1 & -1 & -1 & -1 & 0 & 0 & 1 & 1 & 1 & 2 & 0 & 0 & 0 & 1 \\
1 & 1 & 0 & 2 & 1 & 1 & 1 & 0 & 1 & 1 & 0 & 1 & 3 & 2 & 1 & 1 & 0 & 0 & 0 & -1 & 0 & 1 & 1 & 0 \\
1 & 1 & 1 & 1 & 1 & 1 & 1 & 1 & 1 & 1 & 1 & 1 & 1 & 1 & 1 & 1 & 1 & 1 & 1 & 1 & 1 & 1 & 1 & 1
\end{array}\right) .
$$


From $G_{t}$, we can get the GLSM fields associated to each point are shown in (4.91), where

$$
q=\left\{q_{1}, q_{2}\right\}, r=\left\{r_{1}, \ldots, r_{6}\right\}, s=\left\{s_{1}, \ldots, s_{11}\right\} .
$$

From $Q_{t}$ (and $Q_{F}$ ), the mesonic symmetry reads $\mathrm{U}(1)^{2} \times \mathrm{U}(1)_{\mathrm{R}}$ and the baryonic symmetry reads $\mathrm{U}(1)_{\mathrm{h}}^{4} \times \mathrm{U}(1)^{2}$, where the subscripts "R" and "h" indicate $\mathrm{R}$ - and hidden symmetries respectively.

The Hilbert series of the toric cone is

$$
\begin{aligned}
H S= & \frac{1}{\left(1-t_{2}\right)\left(1-\frac{1}{t_{1} t_{2}^{2}}\right)\left(1-t_{1} t_{2} t_{3}\right)}+\frac{1}{\left(1-\frac{1}{t_{2}}\right)\left(1-\frac{t_{1}}{t_{2}}\right)\left(1-\frac{t_{2}^{2} t_{3}}{t_{1}}\right)} \\
& +\frac{1}{\left(1-t_{2}\right)\left(1-t_{1} t_{2}^{2}\right)\left(1-\frac{t_{3}}{t_{1} t_{2}^{3}}\right)}+\frac{1}{\left(1-t_{2}\right)\left(1-t_{1} t_{3}^{2}\right)\left(1-\frac{1}{t_{1} t_{2} t_{3}}\right)} \\
& +\frac{1}{\left(1-\frac{1}{t_{2}}\right)\left(1-t_{1} t_{3}^{2}\right)\left(1-\frac{t_{2}}{t_{1} t_{3}}\right)}+\frac{1}{\left(1-\frac{1}{t_{1} t_{3}}\right)\left(1-t_{2} t_{3}\right)\left(1-\frac{t_{1} t_{3}}{t_{2}}\right)} \\
& +\frac{1}{\left(1-\frac{1}{t_{2}}\right)\left(1-\frac{t_{2}}{t_{1}}\right)\left(1-t_{1} t_{3}\right)} .
\end{aligned}
$$

The volume function is then

$$
V=-\frac{b_{1}-12\left(b_{2}+4\right)}{\left(b_{1}+6\right)\left(b_{2}+3\right)\left(b_{1}-2 b_{2}-3\right)\left(b_{1}+3 b_{2}-3\right)} .
$$

Minimizing $V$ yields $V_{\min }=0.156243$ at $b_{1}=-2.854659, b_{2}=-0.172760$. Thus, $a_{\max }=$ 1.600072. Together with the superconformal conditions, we can solve for the R-charges of the bifundamentals. Then the R-charges of GLSM fields should satisfy

$$
\begin{aligned}
& \left(0.843750 p_{2}+0.421875 p_{3}\right) p_{4}^{2}+\left(0.843750 p_{2}^{2}+1.6875 p_{3} p_{2}-1.6875 p_{2}\right. \\
& \left.+0.421875 p_{3}^{2}-0.843750 p_{3}\right) p_{4}=-2.53125 p_{3} p_{2}^{2}-2.53125 p_{3}^{2} p_{2}+5.0625 p_{3} p_{2}-0.800036
\end{aligned}
$$

constrained by $\sum_{i=1}^{4} p_{i}=2$ and $0<p_{i}<2$, with others vanishing.

\subsection{Polytope 17: $L^{5,6,1}$}

The polytope is

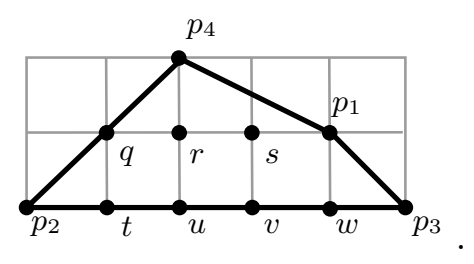


The brane tiling and the corresponding quiver are

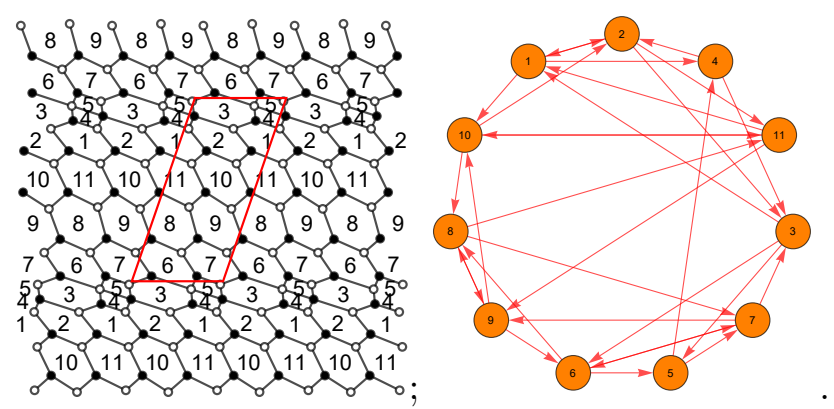

The superpotential is

$$
\begin{aligned}
W= & X_{1,4} X_{4,2} X_{2,1}+X_{2,3} X_{3,1} X_{1,2}+X_{3,6} X_{6,5} X_{5,4} X_{4,3}+X_{5,7} X_{7,3} X_{3,5}+X_{6,8} X_{8,7} X_{7,6} \\
& +X_{7,9} X_{9,6} X_{6,7}+X_{9,10} X_{10,8} X_{8,9}+X_{8,11} X_{11,9} X_{9,8}+X_{11,1} X_{1,10} X_{10,11}+X_{10,2} X_{2,11} X_{11,10} \\
& -X_{2,1} X_{1,10} X_{10,2}-X_{3,1} X_{1,4} X_{4,3}-X_{4,2} X_{2,3} X_{3,5} X_{5,4}-X_{7,3} X_{3,6} X_{6,7}-X_{6,5} X_{5,7} X_{7,6} \\
& -X_{6,8} X_{8,9} X_{9,6}-X_{8,7} X_{7,9} X_{9,8}-X_{11,9} X_{9,10} X_{10,11}-X_{10,8} X_{8,11} X_{11,10}-X_{2,11} X_{11,1} X_{1,2} .
\end{aligned}
$$

The number of perfect matchings is $c=81$, which leads to gigantic $P, Q_{t}$ and $G_{t}$. Hence, we will not list them here. The GLSM fields associated to each point are shown in (4.101), where

$$
\begin{array}{rlrl}
q & =\left\{q_{1}, q_{2}\right\}, & t & =\left\{t_{1}, \ldots, t_{5}\right\}, \\
u & =\left\{u_{1}, \ldots, u_{10}\right\}, & v & \left.=\left\{v_{1}, \ldots, v_{10}\right\}, \ldots, r_{25}\right\}, \quad s=\left\{s_{1}, \ldots, s_{20}\right\} \\
& =\left\{w_{1}, \ldots, w_{5}\right\} .
\end{array}
$$

The mesonic symmetry reads $\mathrm{U}(1)^{2} \times \mathrm{U}(1)_{\mathrm{R}}$ and the baryonic symmetry reads $\mathrm{U}(1)_{\mathrm{h}}^{4} \times \mathrm{U}(1)^{6}$, where the subscripts "R" and "h" indicate R- and hidden symmetries respectively.

The Hilbert series of the toric cone is

$$
\begin{aligned}
H S= & \frac{1}{\left(1-t_{2}\right)\left(1-\frac{t_{2}}{t_{1}}\right)\left(1-\frac{t_{1} t_{3}}{t_{2}^{2}}\right)}+\frac{1}{\left(1-\frac{t_{3}^{2}}{t_{1}}\right)\left(1-t_{2} t_{3}\right)\left(1-\frac{t_{1}}{t_{2} t_{3}^{2}}\right)} \\
& +\frac{1}{\left(1-t_{2}\right)\left(1-\frac{t_{1}}{t_{3}}\right)\left(1-\frac{t_{3}^{2}}{t_{1} t_{2}}\right)}+\frac{1}{\left(1-\frac{t_{1}}{t_{3}^{2}}\right)\left(1-t_{2} t_{3}\right)\left(1-\frac{t_{3}^{2}}{t_{1} t_{2}}\right)} \\
& +\frac{1}{\left(1-\frac{1}{t_{2}}\right)\left(1-\frac{t_{1}}{t_{3}}\right)\left(1-\frac{t_{2} t_{3}^{2}}{t_{1}}\right)}+\frac{1}{\left(1-\frac{1}{t_{2}}\right)\left(1-\frac{t_{2}}{t_{1}}\right)\left(1-t_{1} t_{3}\right)} \\
& +\frac{1}{\left(1-\frac{1}{t_{1}}\right)\left(1-\frac{t_{1}}{t_{2}}\right)\left(1-t_{2} t_{3}\right)}+\frac{1}{\left(1-\frac{1}{t_{1} t_{3}}\right)\left(1-t_{2} t_{3}\right)\left(1-\frac{t_{1} t_{3}}{t_{2}}\right)} \\
& +\frac{1}{\left(1-t_{1}\right)\left(1-\frac{1}{t_{2}}\right)\left(1-\frac{t_{2} t_{3}}{t_{1}}\right)}+\frac{1}{\left(1-t_{2}\right)\left(1-\frac{t_{1}}{t_{2}}\right)\left(1-\frac{t_{3}}{t_{1}}\right)} \\
& +\frac{t_{2}}{\left(1-\frac{t_{3}}{t_{1}}\right)\left(1-t_{2} t_{3}\right)\left(1-\frac{t_{1}}{t_{2} t_{3}}\right)} .
\end{aligned}
$$


The volume function is then

$$
V=-\frac{5 b_{1}-7 b_{2}+24}{\left(b_{2}+3\right)\left(b_{1}-2 b_{2}+3\right)\left(b_{1}-b_{2}+3\right)\left(b_{1}+b_{2}-6\right)} .
$$

Minimizing $V$ yields $V_{\min }=0.0974795$ at $b_{1}=1.8379935, b_{2}=-0.9546900$. Thus, $a_{\max }=$ 2.5646418. Together with the superconformal conditions, we can solve for the R-charges of the bifundamentals. Then the R-charges of GLSM fields should satisfy

$$
\begin{aligned}
& \left(2.8125 p_{2}+0.46875 p_{3}\right) p_{4}^{2}+\left(2.8125 p_{2}^{2}+0.9375 p_{3} p_{2}-5.625 p_{2}+0.46875 p_{3}^{2}-0.9375 p_{3}\right) p_{4} \\
& =-2.34375 p_{3} p_{2}^{2}-2.34375 p_{3}^{2} p_{2}+4.6875 p_{3} p_{2}-1.4248
\end{aligned}
$$

constrained by $\sum_{i=1}^{4} p_{i}=2$ and $0<p_{i}<2$, with others vanishing.

\subsection{Polytope 18: $L^{2,4,1}$}

The polytope is

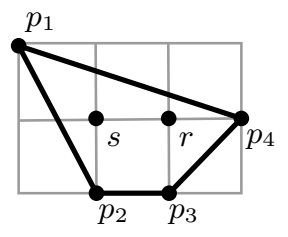

The brane tiling and the corresponding quiver are
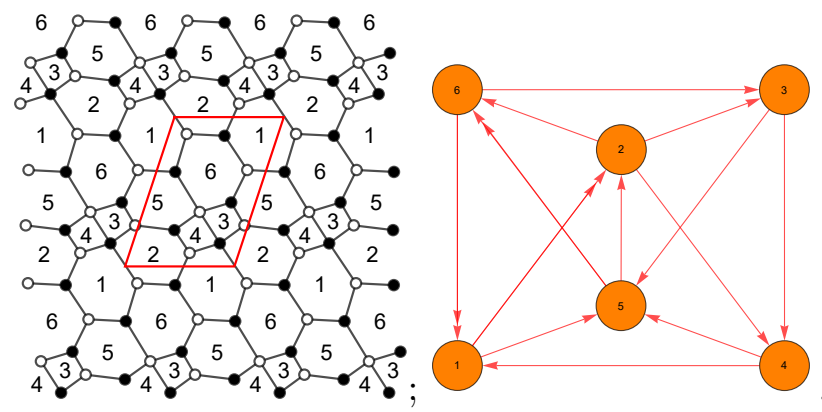

The superpotential is

$$
\begin{aligned}
W= & X_{12}^{1} X_{26} X_{61}^{2}+X_{61}^{1} X_{15} X_{56}^{2}+X_{52} X_{23} X_{35}+X_{63} X_{34} X_{45} X_{56}^{1}+X_{41} X_{12}^{2} X_{24} \\
& -X_{12}^{2} X_{26} X_{61}^{1}-X_{61}^{2} X_{15} X_{56}^{1}-X_{56}^{2} X_{63} X_{35}-X_{23} X_{34} X_{41} X_{12}^{1}-X_{45} X_{52} X_{24}
\end{aligned}
$$


The perfect matching matrix is

$$
P=\left(\begin{array}{c|cccccccccccccccc} 
& r_{1} & s_{1} & r_{2} & s_{2} & s_{3} & r_{3} & p_{1} & p_{2} & p_{3} & s_{4} & s_{5} & r_{4} & r_{5} & p_{4} & s_{6} & r_{6} \\
\hline X_{12}^{1} & 0 & 1 & 0 & 0 & 1 & 0 & 1 & 0 & 0 & 0 & 0 & 0 & 1 & 0 & 0 & 0 \\
X_{12}^{2} & 0 & 1 & 0 & 0 & 1 & 0 & 0 & 1 & 1 & 0 & 0 & 0 & 1 & 0 & 0 & 0 \\
X_{15} & 1 & 1 & 1 & 0 & 0 & 0 & 0 & 0 & 0 & 0 & 0 & 1 & 1 & 1 & 0 & 0 \\
X_{23} & 0 & 0 & 0 & 1 & 0 & 0 & 0 & 1 & 0 & 0 & 0 & 1 & 0 & 0 & 1 & 0 \\
X_{24} & 1 & 0 & 0 & 1 & 0 & 0 & 1 & 0 & 0 & 1 & 0 & 1 & 0 & 0 & 1 & 0 \\
X_{26} & 1 & 0 & 1 & 1 & 0 & 1 & 0 & 0 & 0 & 0 & 0 & 1 & 0 & 1 & 0 & 0 \\
X_{34} & 1 & 0 & 0 & 0 & 0 & 0 & 0 & 0 & 1 & 1 & 0 & 0 & 0 & 0 & 0 & 0 \\
X_{35} & 1 & 1 & 1 & 0 & 0 & 0 & 1 & 0 & 0 & 1 & 1 & 0 & 0 & 0 & 0 & 0 \\
X_{41} & 0 & 0 & 1 & 0 & 0 & 1 & 0 & 0 & 0 & 0 & 1 & 0 & 0 & 1 & 0 & 1 \\
X_{45} & 0 & 1 & 1 & 0 & 0 & 0 & 0 & 1 & 0 & 0 & 1 & 0 & 0 & 0 & 0 & 0 \\
X_{52} & 0 & 0 & 0 & 0 & 1 & 1 & 0 & 0 & 1 & 0 & 0 & 0 & 1 & 1 & 0 & 1 \\
X_{56}^{1} & 0 & 0 & 0 & 1 & 1 & 1 & 1 & 0 & 0 & 0 & 0 & 0 & 0 & 0 & 0 & 0 \\
X_{56}^{2} & 0 & 0 & 0 & 1 & 1 & 1 & 0 & 1 & 1 & 0 & 0 & 0 & 0 & 0 & 0 & 0 \\
X_{61}^{1} & 0 & 0 & 0 & 0 & 0 & 0 & 1 & 0 & 0 & 1 & 1 & 0 & 0 & 0 & 1 & 1 \\
X_{61}^{2} & 0 & 0 & 0 & 0 & 0 & 0 & 0 & 1 & 1 & 1 & 1 & 0 & 0 & 0 & 1 & 1 \\
X_{63} & 0 & 0 & 0 & 0 & 0 & 0 & 0 & 0 & 0 & 0 & 0 & 1 & 1 & 1 & 1 & 1
\end{array}\right),
$$

where the relations between bifundamentals and GLSM fields can be directly read off. Then we can get the total charge matrix:

$$
Q_{t}=\left(\begin{array}{cccccccccccccccc}
r_{1} & s_{1} & r_{2} & s_{2} & s_{3} & r_{3} & p_{1} & p_{2} & p_{3} & s_{4} & s_{5} & r_{4} & r_{5} & p_{4} & s_{6} & r_{6} \\
\hline 0 & 2 & -1 & 2 & -1 & 0 & -1 & -1 & 0 & 0 & 0 & -1 & 0 & 0 & 0 & 1 \\
0 & 1 & 0 & 1 & 0 & 0 & -1 & -1 & 0 & 0 & 0 & -1 & 0 & 0 & 1 & 0 \\
0 & 1 & -1 & 1 & -1 & 0 & 0 & 0 & 0 & 0 & 0 & -1 & 0 & 1 & 0 & 0 \\
0 & 0 & 0 & 1 & -1 & 0 & 0 & 0 & 0 & 0 & 0 & -1 & 1 & 0 & 0 & 0 \\
0 & 1 & -1 & 1 & 0 & 0 & -1 & -1 & 0 & 0 & 1 & 0 & 0 & 0 & 0 & 0 \\
-1 & 1 & 0 & 1 & 0 & 0 & -1 & -1 & 0 & 1 & 0 & 0 & 0 & 0 & 0 & 0 \\
-1 & 1 & 0 & 1 & -1 & 0 & 0 & -1 & 1 & 0 & 0 & 0 & 0 & 0 & 0 & 0 \\
0 & 1 & -1 & 0 & -1 & 1 & 0 & 0 & 0 & 0 & 0 & 0 & 0 & 0 & 0 & 0 \\
\hline 0 & 3 & -1 & 3 & 0 & 0 & -2 & -2 & 0 & 0 & 0 & -1 & 0 & 0 & 0 & 0 \\
0 & 3 & -1 & 1 & -1 & 0 & -1 & -1 & 0 & 0 & 0 & 0 & 0 & 0 & 0 & 0 \\
1 & 2 & -2 & 1 & 0 & 0 & -1 & -1 & 0 & 0 & 0 & 0 & 0 & 0 & 0 & 0 \\
-1 & 2 & -1 & 1 & 0 & 0 & -1 & -1 & 0 & 0 & 0 & 1 & 0 & 0 & 0 & 0 \\
0 & 2 & -1 & 0 & 1 & 0 & -1 & -1 & 0 & 0 & 0 & 0 & 0 & 0 & 0 & 0
\end{array}\right)
$$

with kernel

$$
G_{t}=\left(\begin{array}{cccccccccccccccc}
r_{1} & s_{1} & r_{2} & s_{2} & s_{3} & r_{3} & p_{1} & p_{2} & p_{3} & s_{4} & s_{5} & r_{4} & r_{5} & p_{4} & s_{6} & r_{6} \\
\hline 1 & 0 & 1 & 0 & 0 & 1 & 0 & -1 & 0 & 0 & 0 & 1 & 1 & 2 & 0 & 1 \\
0 & 1 & 0 & 1 & 1 & 0 & 2 & 1 & 0 & 1 & 1 & 0 & 0 & -1 & 1 & 0 \\
1 & 1 & 1 & 1 & 1 & 1 & 1 & 1 & 1 & 1 & 1 & 1 & 1 & 1 & 1 & 1
\end{array}\right)
$$

From $G_{t}$, we can get the GLSM fields associated to each point as shown in (4.108), where

$$
r=\left\{r_{1}, \ldots, r_{6}\right\}, s=\left\{s_{1}, \ldots, s_{6}\right\} .
$$

From $Q_{t}$ (and $Q_{F}$ ), the mesonic symmetry reads $\mathrm{U}(1)^{2} \times \mathrm{U}(1)_{\mathrm{R}}$ and the baryonic symmetry reads $\mathrm{U}(1)_{\mathrm{h}}^{4} \times \mathrm{U}(1)$, where the subscripts " $\mathrm{R}$ " and " $\mathrm{h}$ " indicate $\mathrm{R}$ - and hidden symmetries respectively.

The Hilbert series of the toric cone is

$$
\begin{aligned}
H S= & \frac{1}{\left(1-\frac{1}{t_{1}}\right)\left(1-\frac{1}{t_{1} t_{2}}\right)\left(1-t_{1}^{2} t_{2} t_{3}\right)}+\frac{1}{\left(1-t_{2}\right)\left(1-t_{1} t_{2}\right)\left(1-\frac{t_{3}}{t_{1} t_{2}^{2}}\right)} \\
& +\frac{1}{\left(1-t_{2}\right)\left(1-\frac{t_{1} t_{2}^{2}}{t_{3}}\right)\left(1-\frac{t_{3}^{2}}{t_{1} t_{2}^{3}}\right)}+\frac{1}{\left(1-\frac{1}{t_{2}}\right)\left(1-\frac{t_{1}}{t_{3}}\right)\left(1-\frac{t_{2} t_{3}^{2}}{t_{1}}\right)} \\
& +\frac{1}{\left(1-t_{1}\right)\left(1-\frac{1}{t_{1} t_{2}}\right)\left(1-t_{2} t_{3}\right)}+\frac{1}{\left(1-\frac{1}{t_{2}}\right)\left(1-t_{1} t_{2}\right)\left(1-\frac{t_{3}}{t_{1}}\right)} .
\end{aligned}
$$


The volume function is then

$$
V=-\frac{2\left(b_{1}-7 b_{2}-36\right)}{\left(b_{2}+3\right)\left(b_{1}-b_{2}-6\right)\left(2 b_{1}+b_{2}+3\right)\left(b_{1}+3 b_{2}-6\right)} .
$$

Minimizing $V$ yields $V_{\min }=0.184633$ at $b_{1}=1.260879, b_{2}=-0.213490$. Thus, $a_{\max }=$ 1.354027. Together with the superconformal conditions, we can solve for the R-charges of the bifundamentals. Then the R-charges of GLSM fields should satisfy

$$
\begin{aligned}
& \left(1.6875 p_{3}+4.21875 p_{4}\right) p_{2}^{2}+\left(1.6875 p_{3}^{2}+8.4375 p_{4} p_{3}-3.375 p_{3}\right. \\
& \left.+4.21875 p_{4}^{2}-8.4375 p_{4}\right) p_{2}=-3.375 p_{4} p_{3}^{2}-3.375 p_{4}^{2} p_{3}+6.75 p_{4} p_{3}-0.510277
\end{aligned}
$$

constrained by $\sum_{i=1}^{4} p_{i}=2$ and $0<p_{i}<2$, with others vanishing.

\subsection{Polytope 19: $L^{5,4,1}$}

The polytope is

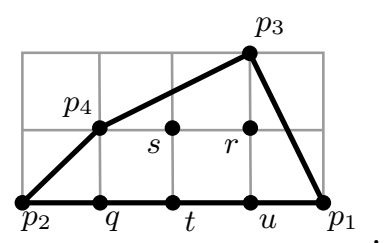

The brane tiling and the corrresponding quiver are
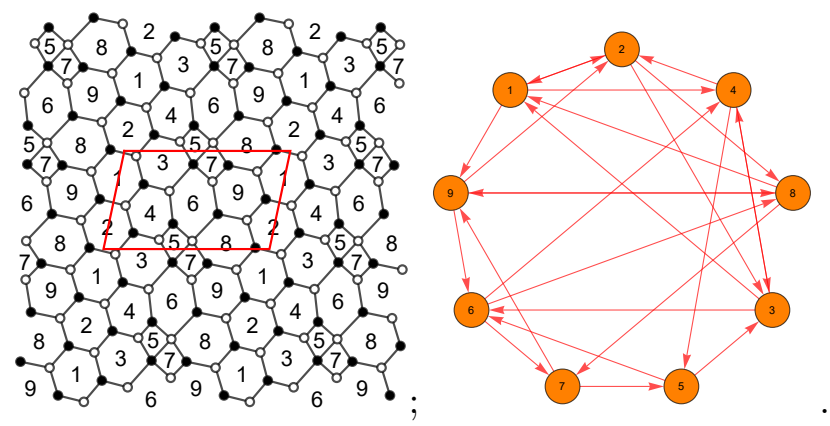

The superpotential is

$$
\begin{aligned}
W= & X_{12} X_{23} X_{31}+X_{21} X_{14} X_{42}+X_{43} X_{36} X_{64}+X_{34} X_{45} X_{53} \\
& +X_{56} X_{68} X_{87} X_{75}+X_{79} X_{96} X_{67}+X_{81} X_{19} X_{98}+X_{92} X_{28} X_{89} \\
& -X_{31} X_{14} X_{43}-X_{42} X_{23} X_{34}-X_{64} X_{45} X_{56}-X_{53} X_{36} X_{67} X_{75} \\
& -X_{96} X_{68} X_{89}-X_{87} X_{79} X_{98}-X_{19} X_{92} X_{21}-X_{28} X_{81} X_{12}
\end{aligned}
$$

The number of perfect matchings is $c=41$, which leads to gigantic $P, Q_{t}$ and $G_{t}$. Hence, we will not list them here. The GLSM fields associated to each point are shown in (4.118), where

$$
\begin{aligned}
q & =\left\{q_{1}, \ldots, q_{4}\right\}, & t & =\left\{t_{1}, \ldots, t_{6}\right\}, \\
s & =\left\{s_{1}, \ldots, s_{14}\right\}, & u & =\left\{u_{1}, \ldots, u_{4}\right\} .
\end{aligned}
$$


The mesonic symmetry reads $\mathrm{U}(1)^{2} \times \mathrm{U}(1)_{\mathrm{R}}$ and the baryonic symmetry reads $\mathrm{U}(1)_{\mathrm{h}}^{4} \times \mathrm{U}(1)^{4}$, where the subscripts " $\mathrm{R}$ " and " $\mathrm{h}$ " indicate $\mathrm{R}$ - and hidden symmetries respectively.

The Hilbert series of the toric cone is

$$
\begin{aligned}
H S= & \frac{1}{\left(1-\frac{t_{1}}{t_{3}}\right)\left(1-\frac{t_{1} t_{2}}{t_{3}}\right)\left(1-\frac{t_{3}^{3}}{t_{1}^{2} t_{2}}\right)}+\frac{1}{\left(1-t_{2}\right)\left(1-\frac{t_{2}}{t_{1}}\right)\left(1-\frac{t_{1} t_{3}}{t_{2}^{2}}\right)} \\
& +\frac{1}{\left(1-t_{1}\right)\left(1-\frac{1}{t_{1} t_{2}}\right)\left(1-t_{2} t_{3}\right)}+\frac{1}{\left(1-t_{1} t_{3}\right)\left(1-t_{2} t_{3}\right)\left(1-\frac{1}{t_{1} t_{2} t_{3}}\right)} \\
& +\frac{1}{\left(1-\frac{t_{1}}{t_{3}}\right)\left(1-t_{2} t_{3}\right)\left(1-\frac{t_{3}}{t_{1} t_{2}}\right)}+\frac{1}{\left(1-\frac{1}{t_{1} t_{3}}\right)\left(1-t_{2} t_{3}\right)\left(1-\frac{t_{1} t_{3}}{t_{2}}\right)} \\
& +\frac{1}{\left(1-\frac{1}{t_{1}}\right)\left(1-\frac{1}{t_{2}}\right)\left(1-t_{1} t_{2} t_{3}\right)}+\frac{1}{\left(1-t_{2}\right)\left(1-\frac{t_{1}}{t_{2}}\right)\left(1-\frac{t_{3}}{t_{1}}\right)} \\
& +\frac{1}{\left(1-\frac{1}{t_{2}}\right)\left(1-t_{1} t_{2}\right)\left(1-\frac{t_{3}}{t_{1}}\right)} .
\end{aligned}
$$

The volume function is then

$$
V=-\frac{8 b_{1}-11 b_{2}+39}{\left(b_{2}+3\right)\left(b_{1}-2 b_{2}+3\right)\left(b_{1}-b_{2}+3\right)\left(2 b_{1}+b_{2}-9\right)} .
$$

Minimizing $V$ yields $V_{\min }=0.120498$ at $b_{1}=0.834510, b_{2}=-0.936102$. Thus, $a_{\max }=$ 2.074723. Together with the superconformal conditions, we can solve for the R-charges of the bifundamentals. Then the R-charges of GLSM fields should satisfy

$$
\begin{aligned}
& \left(6.75 p_{2}+4.21875 p_{4}\right) p_{3}^{2}+\left(6.75 p_{2}^{2}+13.5 p_{4} p_{2}-13.5 p_{2}+4.21875 p_{4}^{2}\right. \\
& \left.-8.4375 p_{4}\right) p_{3}=-3.375 p_{4} p_{2}^{2}-3.375 p_{4}^{2} p_{2}+6.75 p_{4} p_{2}-1.35403
\end{aligned}
$$

constrained by $\sum_{i=1}^{4} p_{i}=2$ and $0<p_{i}<2$, with others vanishing.

\subsection{Polytope 20: $L^{1,5,1} / \mathbb{Z}_{2}(1,0,0,1)$}

The polytope is

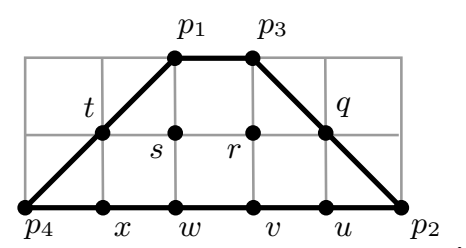

The brane tiling and the corrresponding quiver are
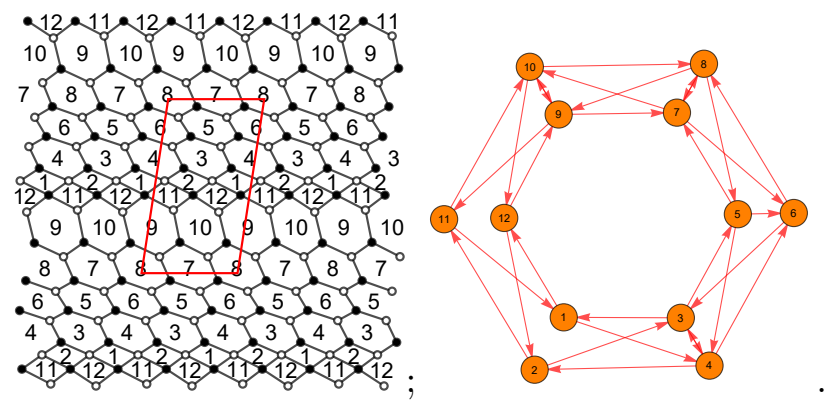
The superpotential is

$$
\begin{aligned}
W= & X_{1,4} X_{4,2} X_{2,11} X_{11,1}+X_{2,3} X_{3,1} X_{1,12} X_{12,2}+X_{3,5} X_{5,4} X_{4,3}+X_{4,6} X_{6,3} X_{3,4}+X_{5,7} X_{7,6} X_{6,5} \\
& +X_{6,8} X_{8,5} X_{5,6}+X_{8,9} X_{9,7} X_{7,8}+X_{7,10} X_{10,8} X_{8,7}+X_{9,11} X_{11,10} X_{10,9}+X_{10,12} X_{12,9} X_{9,10} \\
& -X_{11,10} X_{10,12} X_{12,2} X_{2,11}-X_{3,1} X_{1,4} X_{4,3}-X_{4,2} X_{2,3} X_{3,4}-X_{5,4} X_{4,6} X_{6,5}-X_{6,3} X_{3,5} X_{5,6} \\
& -X_{8,5} X_{5,7} X_{7,8}-X_{7,6} X_{6,8} X_{8,7}-X_{9,7} X_{7,10} X_{10,9}-X_{10,8} X_{8,9} X_{9,10} \\
& -X_{12,9} X_{9,11} X_{11,1} X_{1,12} .
\end{aligned}
$$

The number of perfect matchings is $c=98$, which leads to gigantic $P, Q_{t}$ and $G_{t}$. Hence, we will not list them here. The GLSM fields associated to each point are shown in (4.125), where

$$
\begin{array}{rlrl}
q & =\left\{q_{1}, q_{2}\right\}, & r=\left\{r_{1}, \ldots, r_{30}\right\}, & u=\left\{u_{1}, \ldots, u_{5}\right\}, \quad v=\left\{v_{1}, \ldots, v_{10}\right\}, \\
t=\left\{t_{1}, t_{2}\right\}, & s=\left\{s_{1}, \ldots, s_{30}\right\}, & x=\left\{x_{1}, \ldots, x_{5}\right\}, \quad w=\left\{w_{1}, \ldots, w_{10}\right\} .
\end{array}
$$

The mesonic symmetry reads $\mathrm{U}(1)^{2} \times \mathrm{U}(1)_{\mathrm{R}}$ and the baryonic symmetry reads $\mathrm{U}(1)_{\mathrm{h}}^{4} \times \mathrm{U}(1)^{7}$, where the subscripts "R" and "h" indicate R- and hidden symmetries respectively.

The Hilbert series of the toric cone is

$$
\begin{aligned}
H S= & \frac{1}{\left(1-\frac{1}{t_{2}}\right)\left(1-\frac{t_{3}^{2}}{t_{1}}\right)\left(1-\frac{t_{1} t_{2}}{t_{3}}\right)}+\frac{1}{\left(1-t_{2}\right)\left(1-\frac{t_{1}}{t_{3}}\right)\left(1-\frac{t_{3}^{2}}{t_{1} t_{2}}\right)} \\
& +\frac{1}{\left(1-\frac{t_{1}}{t_{3}^{2}}\right)\left(1-t_{2} t_{3}\right)\left(1-\frac{t_{3}^{2}}{t_{1} t_{2}}\right)}+\frac{1}{\left(1-t_{1}\right)\left(1-t_{2}\right)\left(1-\frac{t_{3}}{t_{1} t_{2}}\right)} \\
& +\frac{1}{\left(1-\frac{1}{t_{1}}\right)\left(1-t_{2}\right)\left(1-\frac{t_{1} t_{3}}{t_{2}}\right)}+\frac{1}{\left(1-t_{1}\right)\left(1-\frac{1}{t_{1} t_{2}}\right)\left(1-t_{2} t_{3}\right)} \\
& +\frac{1}{\left(1-t_{1} t_{3}\right)\left(1-t_{2} t_{3}\right)\left(1-\frac{1}{t_{1} t_{2} t_{3}}\right)}+\frac{1}{\left(1-\frac{t_{1}}{t_{3}}\right)\left(1-t_{2} t_{3}\right)\left(1-\frac{t_{3}}{t_{1} t_{2}}\right)} \\
& +\frac{1}{\left(1-\frac{1}{t_{1} t_{3}}\right)\left(1-t_{2} t_{3}\right)\left(1-\frac{t_{1} t_{3}}{t_{2}}\right)}+\frac{1}{\left(1-\frac{1}{t_{1}}\right)\left(1-\frac{1}{t_{2}}\right)\left(1-t_{1} t_{2} t_{3}\right)} \\
& +\frac{1}{\left(1-\frac{1}{t_{2}}\right)\left(1-t_{1} t_{2}\right)\left(1-\frac{t_{3}}{t_{1}}\right)}+\frac{1}{\left(1-\frac{t_{3}}{t_{1}}\right)\left(1-\frac{t_{3}}{t_{2}}\right)\left(1-\frac{t_{1} t_{2}}{t_{3}}\right)} .
\end{aligned}
$$

The volume function is then

$$
V=-\frac{18-4 b_{2}}{\left(b_{2}-3\right)\left(b_{2}+3\right)\left(-b_{1}+b_{2}-3\right)\left(b_{1}+b_{2}-6\right)} \text {. }
$$

Minimizing $V$ yields $V_{\min }=\frac{4}{225}(-27+7 \sqrt{21})$ at $b_{1}=3 / 2, b_{2}=\frac{1}{2}(3-\sqrt{21})$. Thus, $a_{\max }=(81+21 \sqrt{21}) / 64$. Together with the superconformal conditions, we can solve for the R-charges of the bifundamentals. Then the R-charges of GLSM fields should satisfy

$$
\begin{aligned}
& \left(36 p_{3}+180 p_{4}\right) p_{2}^{2}+\left(36 p_{3}^{2}+72 p_{4} p_{3}-72 p_{3}+180 p_{4}^{2}-360 p_{4}\right) p_{2} \\
& =-36 p_{4} p_{3}^{2}-36 p_{4}^{2} p_{3}+72 p_{4} p_{3}-7 \sqrt{21}-27
\end{aligned}
$$

constrained by $\sum_{i=1}^{4} p_{i}=2$ and $0<p_{i}<2$, with others vanishing. 


\subsection{Polytope 21: $\mathrm{SPP} / \mathbb{Z}_{3}(1,0,0,2)$}

The polytope is

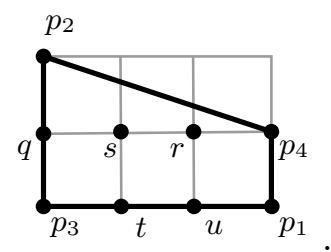

The brane tiling and the corrresponding quiver are
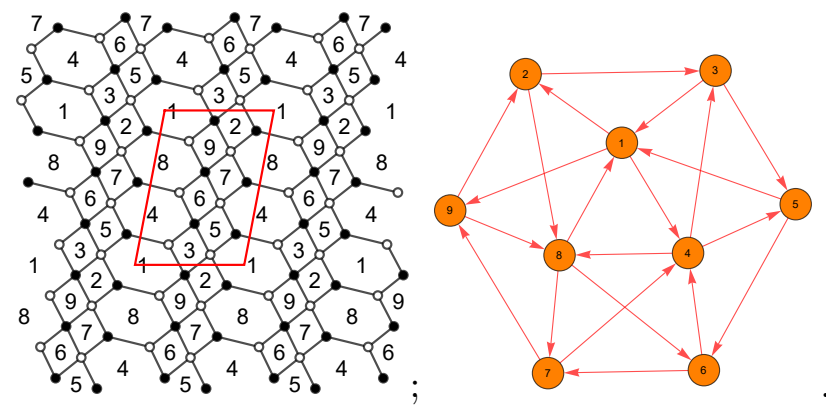

The superpotential is

$$
\begin{aligned}
W= & X_{14} X_{43} X_{31}+X_{23} X_{35} X_{51} X_{12}+X_{48} X_{86} X_{64}+X_{56} X_{67} X_{74} X_{45} \\
& +X_{81} X_{19} X_{98}+X_{79} X_{92} X_{28} X_{87}-X_{19} X_{92} X_{23} X_{31}-X_{28} X_{81} X_{12} \\
& -X_{43} X_{35} X_{56} X_{64}-X_{51} X_{14} X_{45}-X_{86} X_{67} X_{79} X_{98}-X_{74} X_{48} X_{87}
\end{aligned}
$$

The number of perfect matchings is $c=36$, which leads to gigantic $P, Q_{t}$ and $G_{t}$. Hence, we will not list them here. The GLSM fields associated to each point are shown in (4.132), where

$$
\begin{aligned}
q & =\left\{q_{1}, q_{2}\right\}, & r & =\left\{r_{1}, \ldots, r_{15}\right\}, \quad s=\left\{s_{1}, \ldots, s_{9}\right\}, \\
t & =\left\{t_{1}, t_{3}\right\}, & u & =\left\{u_{1}, \ldots, u_{3}\right\} .
\end{aligned}
$$

The mesonic symmetry reads $\mathrm{U}(1)^{2} \times \mathrm{U}(1)_{\mathrm{R}}$ and the baryonic symmetry reads $\mathrm{U}(1)_{\mathrm{h}}^{4} \times \mathrm{U}(1)^{4}$, where the subscripts "R" and "h" indicate R- and hidden symmetries respectively.

The Hilbert series of the toric cone is

$$
\begin{aligned}
H S= & \frac{1}{\left(1-t_{2}\right)\left(1-t_{1} t_{2}\right)\left(1-\frac{t_{3}}{t_{1} t_{2}^{2}}\right)}+\frac{1}{\left(1-t_{2}\right)\left(1-\frac{t_{1} t_{2}^{2}}{t_{3}}\right)\left(1-\frac{t_{3}^{2}}{t_{1} t_{2}^{3}}\right)} \\
& +\frac{1}{\left(1-\frac{1}{t_{2}}\right)\left(1-\frac{t_{3}^{2}}{t_{1}}\right)\left(1-\frac{t_{1} t_{2}}{t_{3}}\right)}+\frac{1}{\left(1-t_{2}\right)\left(1-\frac{1}{t_{1} t_{2}}\right)\left(1-t_{1} t_{3}\right)} \\
& +\frac{1}{\left(1-t_{1}\right)\left(1-\frac{1}{t_{1} t_{2}}\right)\left(1-t_{2} t_{3}\right)}+\frac{1}{\left(1-t_{1} t_{3}\right)\left(1-t_{2} t_{3}\right)\left(1-\frac{1}{t_{1} t_{2} t_{3}}\right)} \\
& +\frac{1}{\left(1-\frac{t_{1}}{t_{3}}\right)\left(1-t_{2} t_{3}\right)\left(1-\frac{t_{3}}{t_{1} t_{2}}\right)}+\frac{1}{\left(1-\frac{1}{t_{1}}\right)\left(1-\frac{1}{t_{2}}\right)\left(1-t_{1} t_{2} t_{3}\right)} \\
& +\frac{1}{\left(1-\frac{1}{t_{2}}\right)\left(1-t_{1} t_{2}\right)\left(1-\frac{t_{3}}{t_{1}}\right)} .
\end{aligned}
$$


The volume function is then

$$
V=-\frac{3\left(b_{1}-15\right)}{\left(b_{1}-6\right)\left(b_{1}+3\right)\left(b_{2}+3\right)\left(b_{1}+3 b_{2}-6\right)} .
$$

Minimizing $V$ yields $V_{\min }=2 \sqrt{3} / 27$ at $b_{1}=3(2-\sqrt{3}), b_{2}=(\sqrt{3}-3) / 2$. Thus, $a_{\max }=$ $(9+\sqrt{3}) / 8$. Together with the superconformal conditions, we can solve for the R-charges of the bifundamentals. Then the R-charges of GLSM fields should satisfy

$$
\begin{aligned}
& \left(162 p_{2}+81 p_{3}\right) p_{4}^{2}+\left(162 p_{2}^{2}+162 p_{3} p_{2}-324 p_{2}+81 p_{3}^{2}-162 p_{3}\right) p_{4} \\
& =-81 p_{3} p_{2}^{2}-81 p_{3}^{2} p_{2}+162 p_{3} p_{2}-4 \sqrt{3}-36
\end{aligned}
$$

constrained by $\sum_{i=1}^{4} p_{i}=2$ and $0<p_{i}<2$, with others vanishing.

\subsection{Polytope 22: $\mathcal{C} /\left(\mathbb{Z}_{3} \times \mathbb{Z}_{2}\right)(1,0,0,2)(0,1,1,0)$}

The polytope is

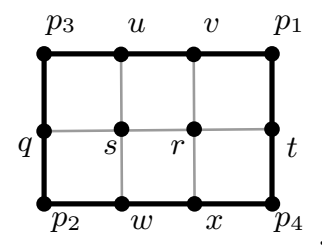

The brane tiling and the corrresponding quiver are
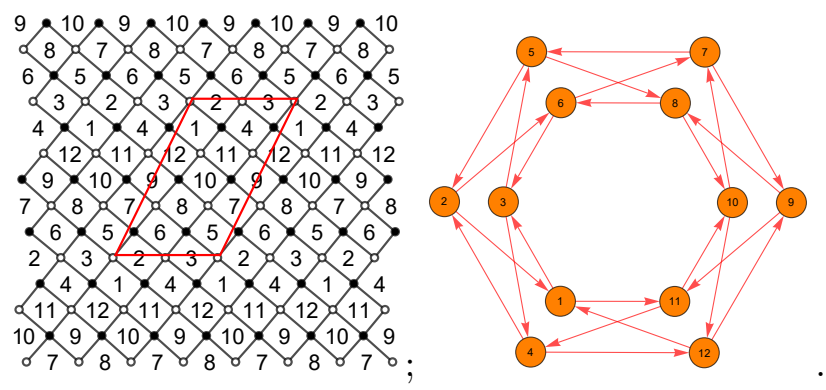

The superpotential is

$$
\begin{aligned}
W= & X_{12,1} X_{1,11} X_{11,10} X_{10,12}+X_{11,4} X_{4,12} X_{12,9} X_{9,11}+X_{3,5} X_{5,2} X_{2,1} X_{1,3}+X_{2,6} X_{6,3} X_{3,4} X_{4,2} \\
& +X_{8,10} X_{10,7} X_{7,5} X_{5,8}+X_{7,9} X_{9,8} X_{8,6} X_{6,7}-X_{1,11} X_{11,4} X_{4,2} X_{2,1}-X_{4,12} X_{12,1} X_{1,3} X_{3,4} \\
& -X_{5,2} X_{2,6} X_{6,7} X_{7,5}-X_{6,3} X_{3,5} X_{5,8} X_{8,6}-X_{10,7} X_{7,9} X_{9,11} X_{11,10} \\
& -X_{9,8} X_{8,10} X_{10,12} X_{12,9} .
\end{aligned}
$$

The number of perfect matchings is $c=80$, which leads to gigantic $P, Q_{t}$ and $G_{t}$. Hence, we will not list them here. The GLSM fields associated to each point are shown in (4.139), where

$$
\begin{aligned}
& q=\left\{q_{1}, q_{2}\right\}, \quad r=\left\{r_{1}, \ldots, r_{30}\right\}, \quad u=\left\{u_{1}, \ldots, u_{3}\right\}, \quad v=\left\{v_{1}, \ldots, v_{3}\right\}, \\
& t=\left\{t_{1}, t_{2}\right\}, \quad s=\left\{s_{1}, \ldots, s_{30}\right\}, \quad w=\left\{w_{1}, \ldots, w_{3}\right\}, \quad x=\left\{x_{1}, \ldots, x_{3}\right\} .
\end{aligned}
$$


The mesonic symmetry reads $\mathrm{U}(1)^{2} \times \mathrm{U}(1)_{\mathrm{R}}$ and the baryonic symmetry reads $\mathrm{U}(1)_{\mathrm{h}}^{4} \times \mathrm{U}(1)^{7}$, where the subscripts "R" and "h" indicate R- and hidden symmetries respectively.

The Hilbert series of the toric cone is

$$
\begin{aligned}
H S= & \frac{1}{\left(1-\frac{1}{t_{2}}\right)\left(1-\frac{t_{3}^{2}}{t_{1}}\right)\left(1-\frac{t_{1} t_{2}}{t_{3}}\right)}+\frac{1}{\left(1-\frac{t_{3}^{2}}{t_{1}}\right)\left(1-\frac{t_{3}}{t_{2}}\right)\left(1-\frac{t_{1} t_{2}}{t_{3}^{2}}\right)} \\
& +\frac{1}{\left(1-t_{2}\right)\left(1-\frac{t_{1}}{t_{3}}\right)\left(1-\frac{t_{3}^{2}}{t_{1} t_{2}}\right)}+\frac{1}{\left(1-t_{2}\right)\left(1-\frac{1}{t_{1} t_{2}}\right)\left(1-t_{1} t_{3}\right)} \\
& +\frac{1}{\left(1-\frac{1}{t_{1}}\right)\left(1-t_{1} t_{2}\right)\left(1-\frac{t_{3}}{t_{2}}\right)}+\frac{1}{\left(1-t_{1}\right)\left(1-t_{2}\right)\left(1-\frac{t_{3}}{t_{1} t_{2}}\right)} \\
& +\frac{1}{\left(1-t_{1}\right)\left(1-\frac{1}{t_{1} t_{2}}\right)\left(1-t_{2} t_{3}\right)}+\frac{1}{\left(1-t_{1} t_{3}\right)\left(1-t_{2} t_{3}\right)\left(1-\frac{1}{t_{1} t_{2} t_{3}}\right)} \\
& +\frac{1}{\left(1-\frac{t_{1}}{t_{3}}\right)\left(1-t_{2} t_{3}\right)\left(1-\frac{t_{3}}{t_{1} t_{2}}\right)}+\frac{1}{\left(1-\frac{1}{t_{1}}\right)\left(1-\frac{1}{t_{2}}\right)\left(1-t_{1} t_{2} t_{3}\right)} \\
& +\frac{1}{\left(1-\frac{1}{t_{2}}\right)\left(1-t_{1} t_{2}\right)\left(1-\frac{t_{3}}{t_{1}}\right)}+\frac{\left.t_{3}\right)\left(1-\frac{t_{3}}{t_{2}}\right)\left(1-\frac{t_{1} t_{2}}{t_{3}}\right)}{\left(1-\frac{t_{3}}{t_{1}}\right)}
\end{aligned}
$$

The volume function is then

$$
V=\frac{18}{\left(b_{1}-6\right)\left(b_{1}+3\right)\left(b_{2}-3\right)\left(b_{2}+3\right)} .
$$

Minimizing $V$ yields $V_{\min }=8 / 81$ at $b_{1}=3 / 2, b_{2}=0$. Thus, $a_{\max }=81 / 32$. Together with the superconformal conditions, we can solve for the R-charges of the bifundamentals, which are $X_{I}=1 / 2$ for any $I$, viz, for all the bifundamentals. Hence, the R-charges of GLSM fields are $p_{i}=1 / 2$ with others vanishing.

\subsection{Polytope 23: $L^{1,3,2}$}

The polytope is

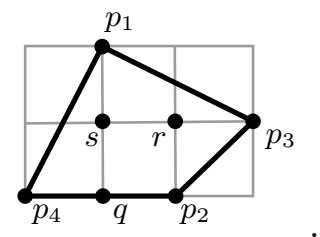

The brane tiling and the corrresponding quiver are
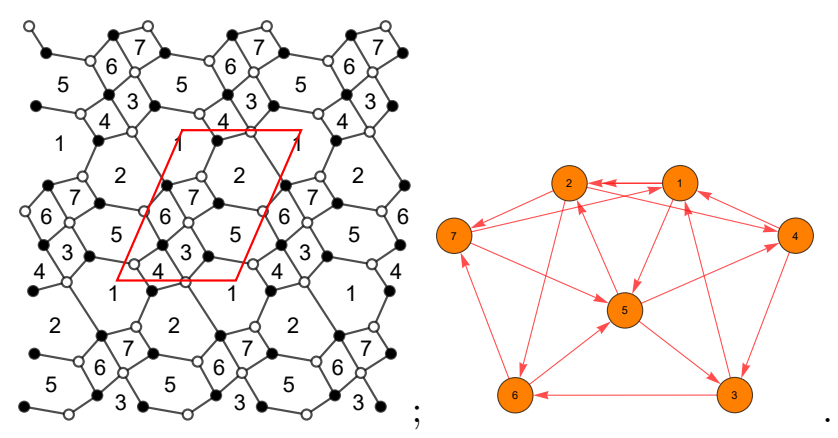
The superpotential is

$$
\begin{aligned}
W= & X_{15} X_{54} X_{41}+X_{24} X_{43} X_{31} X_{12}^{1}+X_{36} X_{67} X_{75} X_{53}+X_{52} X_{26} X_{65}+X_{71} X_{12}^{2} X_{27} \\
& -X_{31} X_{15} X_{53}-X_{12}^{2} X_{24} X_{41}-X_{54} X_{43} X_{36} X_{65}-X_{75} X_{52} X_{27}-X_{26} X_{67} X_{71} X_{12}^{1} .
\end{aligned}
$$

The perfect matching matrix is

$$
P=\left(\begin{array}{c|cccccccccccccccccccc} 
& r_{1} & s_{1} & r_{2} & s_{2} & s_{3} & s_{4} & p_{1} & p_{2} & q_{1} & r_{3} & s_{5} & p_{3} & r_{4} & r_{5} & r_{6} & q_{2} & p_{4} & s_{6} & r_{7} & s_{7} \\
\hline X_{12}^{1} & 0 & 0 & 1 & 0 & 0 & 1 & 1 & 0 & 0 & 0 & 0 & 0 & 0 & 0 & 0 & 0 & 0 & 1 & 0 & 0 \\
X_{12}^{2} & 0 & 0 & 1 & 0 & 0 & 1 & 0 & 1 & 1 & 0 & 0 & 0 & 0 & 0 & 0 & 1 & 1 & 1 & 0 & 0 \\
X_{15} & 1 & 0 & 1 & 0 & 0 & 0 & 0 & 1 & 0 & 1 & 0 & 1 & 0 & 0 & 0 & 1 & 0 & 1 & 1 & 0 \\
X_{24} & 1 & 0 & 0 & 0 & 1 & 0 & 0 & 0 & 0 & 1 & 0 & 1 & 0 & 0 & 1 & 0 & 0 & 0 & 1 & 0 \\
X_{26} & 1 & 1 & 0 & 1 & 1 & 0 & 0 & 0 & 0 & 0 & 0 & 0 & 0 & 0 & 0 & 1 & 1 & 0 & 0 & 0 \\
X_{27} & 1 & 1 & 0 & 1 & 1 & 0 & 1 & 0 & 0 & 1 & 1 & 0 & 0 & 0 & 0 & 0 & 0 & 0 & 0 & 0 \\
X_{31} & 0 & 1 & 0 & 0 & 0 & 0 & 0 & 0 & 1 & 0 & 1 & 0 & 1 & 0 & 0 & 0 & 1 & 0 & 0 & 1 \\
X_{36} & 1 & 1 & 1 & 0 & 0 & 0 & 0 & 0 & 0 & 0 & 0 & 1 & 1 & 0 & 0 & 0 & 0 & 0 & 0 & 0 \\
X_{41} & 0 & 1 & 0 & 1 & 0 & 0 & 1 & 0 & 0 & 0 & 1 & 0 & 1 & 1 & 0 & 0 & 0 & 0 & 0 & 1 \\
X_{43} & 0 & 0 & 0 & 1 & 0 & 0 & 0 & 1 & 0 & 0 & 0 & 0 & 0 & 1 & 0 & 1 & 0 & 0 & 0 & 0 \\
X_{52} & 0 & 0 & 1 & 0 & 0 & 1 & 0 & 1 & 1 & 0 & 0 & 1 & 1 & 1 & 1 & 0 & 0 & 0 & 0 & 0 \\
X_{53} & 0 & 0 & 0 & 1 & 1 & 1 & 1 & 0 & 0 & 0 & 0 & 0 & 0 & 1 & 1 & 0 & 0 & 0 & 0 & 0 \\
X_{54} & 0 & 0 & 0 & 0 & 1 & 1 & 0 & 0 & 1 & 0 & 0 & 0 & 0 & 0 & 1 & 0 & 1 & 0 & 0 & 0 \\
X_{65} & 0 & 0 & 0 & 0 & 0 & 0 & 1 & 0 & 0 & 1 & 1 & 0 & 0 & 0 & 0 & 0 & 0 & 1 & 1 & 1 \\
X_{67} & 0 & 0 & 0 & 0 & 0 & 0 & 0 & 1 & 1 & 1 & 1 & 0 & 0 & 0 & 0 & 0 & 0 & 0 & 0 & 0 \\
X_{71} & 0 & 0 & 0 & 0 & 0 & 0 & 0 & 0 & 0 & 0 & 0 & 1 & 1 & 1 & 1 & 0 & 0 & 0 & 1 & 1 \\
X_{75} & 0 & 0 & 0 & 0 & 0 & 0 & 0 & 0 & 0 & 0 & 0 & 0 & 0 & 0 & 0 & 1 & 1 & 1 & 1 & 1
\end{array}\right)
$$

where the relations between bifundamentals and GLSM fields can be directly read off. Then we can get the total charge matrix:

$$
Q_{t}=\left(\begin{array}{cccccccccccccccccccc}
r_{1} & s_{1} & r_{2} & s_{2} & s_{3} & s_{4} & p_{1} & p_{2} & q_{1} & r_{3} & s_{5} & p_{3} & r_{4} & r_{5} & r_{6} & q_{2} & p_{4} & s_{6} & r_{7} & s_{7} \\
\hline 1 & -1 & 1 & 1 & 0 & 0 & -1 & 0 & 0 & 0 & 0 & -1 & 0 & 0 & 0 & -1 & 0 & 0 & 0 & 1 \\
0 & 0 & 1 & 1 & 0 & 0 & -1 & 0 & 0 & 0 & 0 & -1 & 0 & 0 & 0 & -1 & 0 & 0 & 1 & 0 \\
0 & 0 & 0 & 1 & 0 & 0 & -1 & 0 & 0 & 0 & 0 & 0 & 0 & 0 & 0 & -1 & 0 & 1 & 0 & 0 \\
1 & -1 & 0 & 1 & -1 & 0 & 0 & 0 & 0 & 0 & 0 & 0 & 0 & 0 & 0 & -1 & 1 & 0 & 0 & 0 \\
1 & 0 & 0 & 0 & -1 & 0 & 0 & 0 & 0 & 0 & 0 & -1 & 0 & 0 & 1 & 0 & 0 & 0 & 0 & 0 \\
1 & 0 & 0 & -1 & 0 & 0 & 0 & 0 & 0 & 0 & 0 & -1 & 0 & 1 & 0 & 0 & 0 & 0 & 0 & 0 \\
1 & -1 & 0 & 0 & 0 & 0 & 0 & 0 & 0 & 0 & 0 & -1 & 1 & 0 & 0 & 0 & 0 & 0 & 0 & 0 \\
0 & -1 & 1 & 1 & 0 & 0 & -1 & -1 & 0 & 0 & 1 & 0 & 0 & 0 & 0 & 0 & 0 & 0 & 0 & 0 \\
-1 & 0 & 1 & 1 & 0 & 0 & -1 & -1 & 0 & 1 & 0 & 0 & 0 & 0 & 0 & 0 & 0 & 0 & 0 & 0 \\
1 & -1 & 0 & 1 & -1 & 0 & 0 & -1 & 1 & 0 & 0 & 0 & 0 & 0 & 0 & 0 & 0 & 0 & 0 & 0 \\
1 & 0 & -1 & 0 & -1 & 1 & 0 & 0 & 0 & 0 & 0 & 0 & 0 & 0 & 0 & 0 & 0 & 0 & 0 & 0 \\
\hline 2 & -1 & 1 & 0 & 0 & 0 & 0 & 1 & 0 & 0 & 0 & -2 & 0 & 0 & 0 & -1 & 0 & 0 & 0 & 0 \\
1 & -1 & 2 & 1 & 0 & 0 & -1 & -1 & 0 & 0 & 0 & -1 & 0 & 0 & 0 & 0 & 0 & 0 & 0 & 0 \\
2 & -1 & 0 & -1 & -1 & 0 & 1 & 0 & 0 & 0 & 0 & -1 & 0 & 0 & 0 & 1 & 0 & 0 & 0 & 0 \\
1 & -1 & 1 & -1 & 1 & 0 & 0 & 0 & 0 & 0 & 0 & -1 & 0 & 0 & 0 & 0 & 0 & 0 & 0 & 0 \\
1 & -2 & 1 & 1 & 0 & 0 & 0 & 0 & 0 & 0 & 0 & -1 & 0 & 0 & 0 & 0 & 0 & 0 & 0 & 0 \\
0 & -1 & 2 & 0 & 0 & 0 & 0 & 0 & 0 & 0 & 0 & -1 & 0 & 0 & 0 & 0 & 0 & 0 & 0 & 0
\end{array}\right)
$$

with kernel

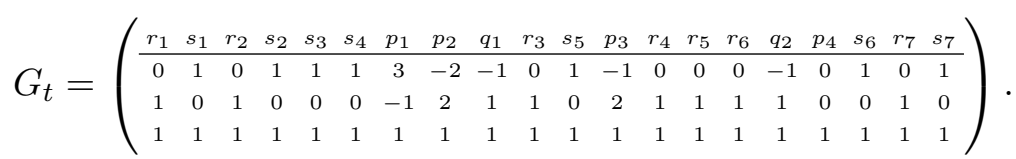

From $G_{t}$, we can get the GLSM fields associated to each point as shown in (4.145), where

$$
q=\left\{q_{1}, q_{2}\right\}, \quad r=\left\{r_{1}, \ldots, r_{7}\right\}, \quad s=\left\{s_{1}, \ldots, s_{7}\right\} .
$$

From $Q_{t}$ (and $\left.Q_{F}\right)$, the mesonic symmetry reads $\mathrm{U}(1)^{2} \times \mathrm{U}(1)_{\mathrm{R}}$ and the baryonic symmetry reads $\mathrm{U}(1)_{\mathrm{h}}^{4} \times \mathrm{U}(1)^{2}$, where the subscripts " $\mathrm{R}$ " and " $\mathrm{h}$ " indicate $\mathrm{R}$ - and hidden symmetries respectively. 
The Hilbert series of the toric cone is

$$
\begin{aligned}
H S= & \frac{1}{\left(1-\frac{1}{t_{1}}\right)\left(1-\frac{t_{2}}{t_{1}}\right)\left(1-\frac{t_{1}^{2} t_{3}}{t_{2}}\right)}+\frac{1}{\left(1-t_{2}\right)\left(1-\frac{t_{1} t_{2}}{t_{3}}\right)\left(1-\frac{t_{3}^{2}}{t_{1} t_{2}^{2}}\right)} \\
& +\frac{1}{\left(1-\frac{1}{t_{2}}\right)\left(1-\frac{t_{1}}{t_{3}}\right)\left(1-\frac{t_{2} t_{3}^{2}}{t_{1}}\right)}+\frac{1}{\left(1-t_{1}\right)\left(1-t_{2}\right)\left(1-\frac{t_{3}}{t_{1} t_{2}}\right)} \\
& +\frac{1}{\left(1-t_{1}\right)\left(1-\frac{1}{t_{1} t_{2}}\right)\left(1-t_{2} t_{3}\right)}+\frac{1}{\left(1-\frac{1}{t_{1}}\right)\left(1-\frac{t_{1}}{t_{2}}\right)\left(1-t_{2} t_{3}\right)} \\
& +\frac{1}{\left(1-\frac{1}{t_{2}}\right)\left(1-t_{1} t_{2}\right)\left(1-\frac{t_{3}}{t_{1}}\right)} .
\end{aligned}
$$

The volume function is then

$$
V=-\frac{4 b_{1}-7 b_{2}-69}{\left(b_{2}+3\right)\left(-2 b_{1}+b_{2}-3\right)\left(-b_{1}+b_{2}+6\right)\left(b_{1}+2 b_{2}-6\right)} .
$$

Minimizing $V$ yields $V_{\min }=0.165004$ at $b_{1}=1.201482, b_{2}=-0.491432$. Thus, $a_{\max }=$ 1.515115. Together with the superconformal conditions, we can solve for the R-charges of the bifundamentals. Then the R-charges of GLSM fields should satisfy

$$
\begin{aligned}
& \left(0.50625 p_{3}+0.675 p_{4}\right) p_{2}^{2}+\left(0.50625 p_{3}^{2}+1.6875 p_{4} p_{3}-1.0125 p_{3}+0.675 p_{4}^{2}-1.35 p_{4}\right) p_{2} \\
& =-0.84375 p_{4} p_{3}^{2}-0.84375 p_{4}^{2} p_{3}+1.6875 p_{4} p_{3}-0.303023
\end{aligned}
$$

constrained by $\sum_{i=1}^{4} p_{i}=2$ and $0<p_{i}<2$, with others vanishing.

\subsection{Polytope 24: $\mathcal{C} / \mathbb{Z}_{4}(0,1,2,1)$}

The polytope is

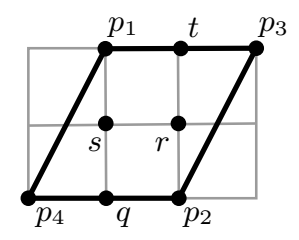

The brane tiling and the corrresponding quiver are
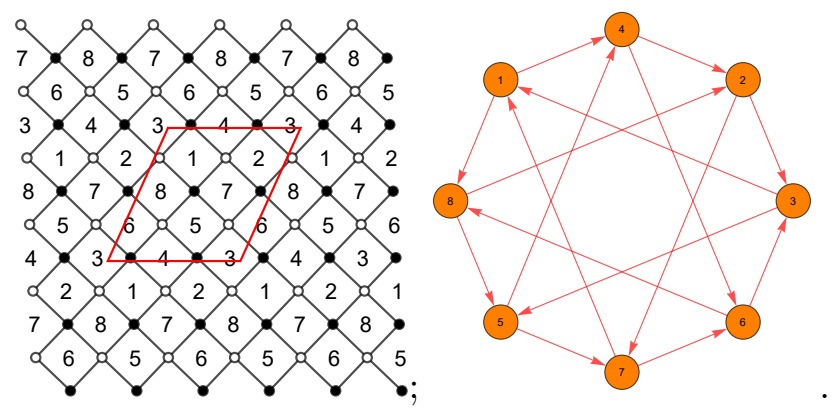
The superpotential is

$$
\begin{aligned}
W= & X_{23} X_{31} X_{18} X_{82}+X_{14} X_{42} X_{27} X_{71}+X_{57} X_{76} X_{63} X_{35}+X_{68} X_{85} X_{54} X_{46} \\
& -X_{31} X_{14} X_{46} X_{63}-X_{42} X_{23} X_{35} X_{54}-X_{85} X_{57} X_{71} X_{18}-X_{76} X_{68} X_{82} X_{27}
\end{aligned}
$$

The perfect matching matrix is

$$
P=\left(\begin{array}{c|cccccccccccccccccccccccc} 
& t_{1} & r_{1} & s_{1} & p_{1} & r_{2} & p_{2} & q_{1} & s_{2} & r_{3} & r_{4} & s_{3} & s_{4} & r_{5} & s_{5} & r_{6} & r_{7} & p_{3} & t_{2} & s_{6} & s_{7} & q_{2} & p_{4} & r_{8} & s_{8} \\
\hline X_{14} & 0 & 0 & 0 & 0 & 1 & 1 & 0 & 0 & 0 & 0 & 0 & 1 & 0 & 0 & 0 & 1 & 0 & 0 & 0 & 0 & 1 & 0 & 1 & 0 \\
X_{18} & 1 & 0 & 0 & 1 & 1 & 0 & 0 & 1 & 0 & 0 & 1 & 1 & 0 & 0 & 0 & 0 & 0 & 0 & 0 & 0 & 0 & 0 & 0 & 0 \\
X_{23} & 0 & 1 & 0 & 0 & 0 & 1 & 0 & 0 & 0 & 1 & 0 & 0 & 1 & 0 & 0 & 0 & 0 & 0 & 0 & 1 & 1 & 0 & 0 & 0 \\
X_{27} & 1 & 1 & 1 & 1 & 0 & 0 & 0 & 0 & 0 & 0 & 0 & 0 & 0 & 0 & 0 & 0 & 0 & 0 & 1 & 1 & 0 & 0 & 0 & 0 \\
X_{31} & 0 & 0 & 1 & 0 & 0 & 0 & 1 & 0 & 1 & 0 & 0 & 0 & 0 & 1 & 0 & 0 & 0 & 0 & 1 & 0 & 0 & 1 & 0 & 0 \\
X_{35} & 1 & 0 & 0 & 0 & 1 & 0 & 0 & 0 & 1 & 0 & 0 & 0 & 0 & 0 & 0 & 0 & 1 & 0 & 1 & 0 & 0 & 0 & 1 & 0 \\
X_{42} & 0 & 0 & 0 & 0 & 0 & 0 & 1 & 1 & 0 & 0 & 1 & 0 & 0 & 0 & 1 & 0 & 0 & 0 & 0 & 0 & 0 & 1 & 0 & 1 \\
X_{46} & 1 & 1 & 0 & 0 & 0 & 0 & 0 & 0 & 0 & 0 & 1 & 0 & 1 & 0 & 1 & 0 & 1 & 0 & 0 & 0 & 0 & 0 & 0 & 0 \\
X_{54} & 0 & 0 & 1 & 1 & 0 & 0 & 0 & 0 & 0 & 0 & 0 & 1 & 0 & 1 & 0 & 1 & 0 & 1 & 0 & 0 & 0 & 0 & 0 & 0 \\
X_{57} & 0 & 1 & 1 & 0 & 0 & 1 & 1 & 0 & 0 & 0 & 0 & 0 & 0 & 0 & 1 & 1 & 0 & 0 & 0 & 0 & 0 & 0 & 0 & 0 \\
X_{63} & 0 & 0 & 0 & 1 & 0 & 0 & 0 & 1 & 0 & 1 & 0 & 0 & 0 & 0 & 0 & 0 & 0 & 1 & 0 & 1 & 0 & 0 & 0 & 1 \\
X_{68} & 0 & 0 & 0 & 0 & 1 & 1 & 1 & 1 & 1 & 1 & 0 & 0 & 0 & 0 & 0 & 0 & 0 & 0 & 0 & 0 & 0 & 0 & 0 & 0 \\
X_{71} & 0 & 0 & 0 & 0 & 0 & 0 & 0 & 0 & 1 & 1 & 0 & 0 & 1 & 1 & 0 & 0 & 1 & 1 & 0 & 0 & 0 & 0 & 0 & 0 \\
X_{76} & 0 & 0 & 0 & 0 & 0 & 0 & 0 & 0 & 0 & 0 & 1 & 1 & 1 & 1 & 0 & 0 & 0 & 0 & 0 & 0 & 1 & 1 & 0 & 0 \\
X_{82} & 0 & 0 & 0 & 0 & 0 & 0 & 0 & 0 & 0 & 0 & 0 & 0 & 0 & 0 & 1 & 1 & 1 & 1 & 0 & 0 & 0 & 0 & 1 & 1 \\
X_{85} & 0 & 0 & 0 & 0 & 0 & 0 & 0 & 0 & 0 & 0 & 0 & 0 & 0 & 0 & 0 & 0 & 0 & 0 & 1 & 1 & 1 & 1 & 1 & 1
\end{array}\right),
$$

where the relations between bifundamentals and GLSM fields can be directly read off. Then we can get the total charge matrix:

$$
Q_{t}=\left(\begin{array}{cccccccccccccccccccccccc}
t_{1} & r_{1} & s_{1} & p_{1} & r_{2} & p_{2} & q_{1} & s_{2} & r_{3} & r_{4} & s_{3} & s_{4} & r_{5} & s_{5} & r_{6} & r_{7} & p_{3} & t_{2} & s_{6} & s_{7} & q_{2} & p_{4} & r_{8} & s_{8} \\
\hline 1 & 0 & 1 & -1 & 0 & 0 & 0 & 0 & 0 & 0 & 0 & 0 & 0 & 0 & -1 & 0 & 0 & 0 & -1 & 0 & 0 & 0 & 0 & 1 \\
1 & 0 & 0 & 0 & -1 & 0 & 1 & 0 & 0 & 0 & 0 & 0 & 0 & 0 & -1 & 0 & 0 & 0 & -1 & 0 & 0 & 0 & 1 & 0 \\
1 & 0 & 0 & 0 & 0 & 0 & 0 & 0 & 0 & 0 & -1 & 0 & 0 & 0 & 0 & 0 & 0 & 0 & -1 & 0 & 0 & 1 & 0 & 0 \\
2 & -1 & 0 & 0 & -1 & 0 & 1 & 0 & 0 & 0 & -1 & 0 & 0 & 0 & 0 & 0 & 0 & 0 & -1 & 0 & 1 & 0 & 0 & 0 \\
1 & -1 & 1 & -1 & 0 & 0 & 0 & 0 & 0 & 0 & 0 & 0 & 0 & 0 & 0 & 0 & 0 & 0 & -1 & 1 & 0 & 0 & 0 & 0 \\
1 & 0 & 0 & -1 & 0 & 0 & 1 & 0 & -1 & 0 & 0 & 0 & 0 & 0 & -1 & 0 & 0 & 1 & 0 & 0 & 0 & 0 & 0 & 0 \\
0 & 0 & 0 & 0 & 0 & 0 & 1 & 0 & -1 & 0 & 0 & 0 & 0 & 0 & -1 & 0 & 1 & 0 & 0 & 0 & 0 & 0 & 0 & 0 \\
1 & 0 & -1 & 0 & -1 & 0 & 1 & 0 & 0 & 0 & 0 & 0 & 0 & 0 & -1 & 1 & 0 & 0 & 0 & 0 & 0 & 0 & 0 & 0 \\
1 & 0 & -1 & 0 & 0 & 0 & 1 & 0 & -1 & 0 & -1 & 0 & 0 & 1 & 0 & 0 & 0 & 0 & 0 & 0 & 0 & 0 & 0 & 0 \\
1 & -1 & 0 & 0 & 0 & 0 & 1 & 0 & -1 & 0 & -1 & 0 & 1 & 0 & 0 & 0 & 0 & 0 & 0 & 0 & 0 & 0 & 0 & 0 \\
1 & 0 & -1 & 0 & -1 & 0 & 1 & 0 & 0 & 0 & -1 & 1 & 0 & 0 & 0 & 0 & 0 & 0 & 0 & 0 & 0 & 0 & 0 & 0 \\
1 & -1 & 1 & -1 & 0 & 0 & 0 & 0 & -1 & 1 & 0 & 0 & 0 & 0 & 0 & 0 & 0 & 0 & 0 & 0 & 0 & 0 & 0 & 0 \\
0 & 0 & 1 & -1 & 0 & 0 & -1 & 1 & 0 & 0 & 0 & 0 & 0 & 0 & 0 & 0 & 0 & 0 & 0 & 0 & 0 & 0 & 0 & 0 \\
1 & -1 & 0 & 0 & -1 & 1 & 0 & 0 & 0 & 0 & 0 & 0 & 0 & 0 & 0 & 0 & 0 & 0 & 0 & 0 & 0 & 0 & 0 & 0 \\
\hline 1 & 0 & 0 & 0 & 1 & 0 & 1 & 0 & -1 & 0 & 0 & 0 & 0 & 0 & -1 & 0 & 0 & 0 & -1 & 0 & 0 & 0 & 0 & 0 \\
1 & 0 & 0 & 0 & 1 & 0 & 1 & 0 & -2 & 0 & -1 & 0 & 0 & 0 & 0 & 0 & 0 & 0 & 0 & 0 & 0 & 0 & 0 & 0 \\
0 & 0 & 1 & -1 & 1 & 0 & -1 & 0 & -1 & 0 & 1 & 0 & 0 & 0 & 0 & 0 & 0 & 0 & 0 & 0 & 0 & 0 & 0 & 0 \\
0 & 0 & -1 & 0 & 1 & 0 & 0 & 0 & -1 & 0 & 0 & 0 & 0 & 0 & 0 & 0 & 0 & 0 & 1 & 0 & 0 & 0 & 0 & 0 \\
-1 & 0 & 1 & 0 & 2 & 0 & -1 & 0 & -1 & 0 & 0 & 0 & 0 & 0 & 0 & 0 & 0 & 0 & 0 & 0 & 0 & 0 & 0 & 0 \\
-1 & 1 & -1 & 1 & 1 & 0 & 0 & 0 & -1 & 0 & 0 & 0 & 0 & 0 & 0 & 0 & 0 & 0 & 0 & 0 & 0 & 0 & 0 & 0 \\
0 & -1 & 0 & 0 & 1 & 0 & 0 & 0 & -1 & 0 & 0 & 0 & 0 & 0 & 1 & 0 & 0 & 0 & 0 & 0 & 0 & 0 & 0 & 0
\end{array}\right)
$$

with kernel

$$
G_{t}=\left(\begin{array}{cccccccccccccccccccccccccc}
t_{1} & r_{1} & s_{1} & p_{1} & r_{2} & p_{2} & q_{1} & s_{2} & r_{3} & r_{4} & s_{3} & s_{4} & r_{5} & s_{5} & r_{6} & r_{7} & p_{3} & t_{2} & s_{6} & s_{7} & q_{2} & p_{4} & r_{8} & s_{8} \\
\hline 2 & 0 & 1 & 3 & 0 & -2 & -1 & 1 & 0 & 0 & 1 & 1 & 0 & 1 & 0 & 0 & 1 & 2 & 1 & 1 & -1 & 0 & 0 & 1 \\
0 & 1 & 0 & -1 & 1 & 2 & 1 & 0 & 1 & 1 & 0 & 0 & 1 & 0 & 1 & 1 & 1 & 0 & 0 & 0 & 1 & 0 & 1 & 0 \\
1 & 1 & 1 & 1 & 1 & 1 & 1 & 1 & 1 & 1 & 1 & 1 & 1 & 1 & 1 & 1 & 1 & 1 & 1 & 1 & 1 & 1 & 1 & 1
\end{array}\right)
$$

From $G_{t}$, we can get the GLSM fields associated to each point as shown in (4.155), where

$$
q=\left\{q_{1}, q_{2}\right\}, \quad r=\left\{r_{1}, \ldots, r_{8}\right\}, \quad s=\left\{s_{1}, \ldots, s_{8}\right\}, \quad t=\left\{t_{1}, t_{2}\right\} .
$$


From $Q_{t}$ (and $Q_{F}$ ), the mesonic symmetry reads $\mathrm{U}(1)^{2} \times \mathrm{U}(1)_{\mathrm{R}}$ and the baryonic symmetry reads $\mathrm{U}(1)_{\mathrm{h}}^{4} \times \mathrm{U}(1)^{3}$, where the subscripts " $\mathrm{R}$ " and " $\mathrm{h}$ " indicate $\mathrm{R}$ - and hidden symmetries respectively.

The Hilbert series of the toric cone is

$$
\begin{aligned}
H S= & \frac{1}{\left(1-\frac{t_{1}}{t_{3}}\right)\left(1-\frac{t_{1}}{t_{2} t_{3}}\right)\left(1-\frac{t_{2} t_{3}^{3}}{t_{1}^{2}}\right)}+\frac{1}{\left(1-\frac{1}{t_{1}}\right)\left(1-\frac{t_{2}}{t_{1}}\right)\left(1-\frac{t_{1}^{2} t_{3}}{t_{2}}\right)} \\
& +\frac{1}{\left(1-t_{1}\right)\left(1-t_{2}\right)\left(1-\frac{t_{3}}{t_{1} t_{2}}\right)}+\frac{1}{\left(1-\frac{1}{t_{1}}\right)\left(1-\frac{t_{1}}{t_{2}}\right)\left(1-t_{2} t_{3}\right)} \\
& +\frac{1}{\left(1-\frac{t_{3}}{t_{1}}\right)\left(1-t_{2} t_{3}\right)\left(1-\frac{t_{1}}{t_{2} t_{3}}\right)}+\frac{1}{\left(1-t_{1}\right)\left(1-\frac{1}{t_{2}}\right)\left(1-\frac{t_{2} t_{3}}{t_{1}}\right)} \\
& +\frac{1}{\left(1-\frac{t_{3}}{t_{1}}\right)\left(1-\frac{t_{3}}{t_{2}}\right)\left(1-\frac{t_{1} t_{2}}{t_{3}}\right)}+\frac{\left.1-\frac{t_{1}}{t_{3}}\right)\left(1-\frac{t_{3}}{t_{2}}\right)\left(1-\frac{t_{2} t_{3}}{t_{1}}\right)}{(1-}
\end{aligned}
$$

The volume function is then

$$
V=\frac{48}{\left(b_{2}-3\right)\left(b_{2}+3\right)\left(-2 b_{1}+b_{2}-3\right)\left(-2 b_{1}+b_{2}+9\right)} .
$$

Minimizing $V$ yields $V_{\min }=4 / 27$ at $b_{1}=3 / 2, b_{2}=0$. Thus, $a_{\max }=27 / 16$. Together with the superconformal conditions, we can solve for the R-charges of the bifundamentals, which are $X_{I}=1 / 2$ for any $I$, viz, for all the bifundamentals. Hence, the R-charges of GLSM fields are $p_{i}=1 / 2$ with others vanishing.

\section{Sixteen pentagons}

For brevity, we will use $K^{a, b, c, d}$ to denote a special family of cones. In analogy to defining $X^{p, q}$ from unhiggsing $Y^{p, q}$ and $Y^{p, q-1}$ in [35], $K^{a, b, c, d}$ corresponds to the toric diagram

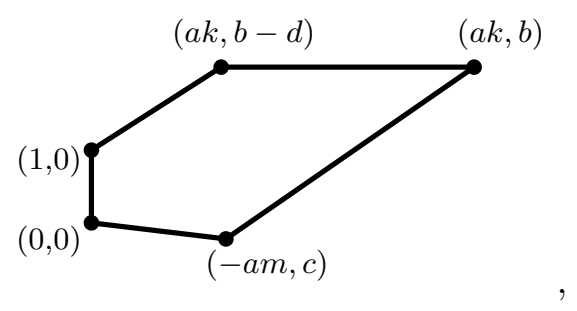

where $b m+c k=1$ and $b \geq d$, such that it can be blown down to $L^{a, b, c}$ (and more if $m=$ 0 ) [34]. Here, we will drop the condition that $a, c \leq b$ inherited from $L^{a, b, c}$ since for instance, if $a>b$, we could write $L^{b, a, c}$. Also, when $m=0$, for simplicity, let us forget about the condition that $\operatorname{gcd}(a, b, c, a+b-c)=1$ (and so forth), which makes the baryonic $\mathrm{U}(1)$ action specified by such GLSM charges effective, since we still have other higgsed singularities among these $L$ 's. Then in particular, for example, we have $K^{p+q-1, p-q+1, p, 1}=X^{p, q}$. 


\subsection{Polytope 25: $X^{3,2}$}

The polytope is

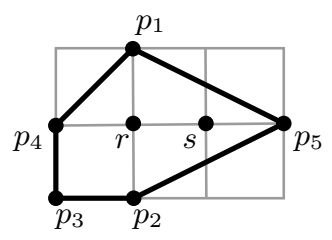

The brane tiling and the corrresponding quiver are
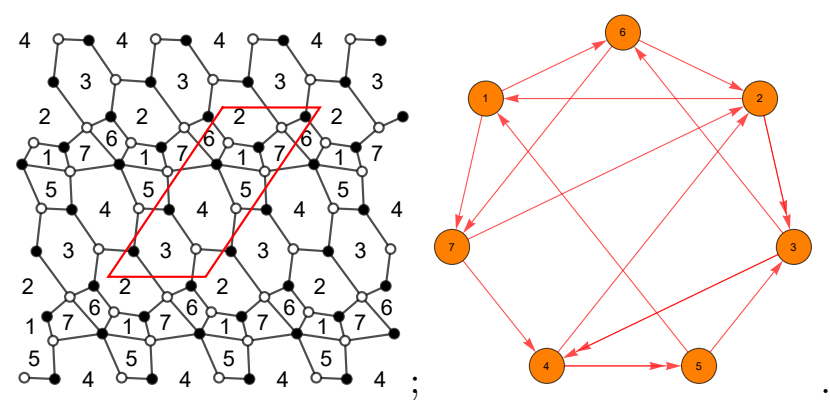

The superpotential is

$$
\begin{aligned}
W= & X_{17} X_{74} X_{45}^{2} X_{51}+X_{53} X_{34}^{2} X_{45}^{1}+X_{34}^{1} X_{42} X_{23}^{2}+X_{36} X_{67} X_{72} X_{23}^{1} \\
& +X_{21} X_{16} X_{62}-X_{16} X_{67} X_{74} X_{45}^{1} X_{51}-X_{45}^{2} X_{53} X_{34}^{1}-X_{34}^{2} X_{42} X_{23}^{1} \\
& -X_{23}^{2} X_{36} X_{62}-X_{72} X_{21} X_{17} .
\end{aligned}
$$

The perfect matching matrix is

$$
P=\left(\begin{array}{c|ccccccccccccccccccccc} 
& r_{1} & r_{2} & s_{1} & r_{3} & r_{4} & s_{2} & r_{5} & s_{3} & p_{1} & p_{2} & r_{6} & s_{4} & p_{3} & r_{7} & p_{4} & r_{8} & s_{5} & s_{6} & p_{5} & r_{9} & s_{7} \\
\hline X_{16} & 1 & 0 & 0 & 0 & 1 & 1 & 0 & 0 & 0 & 0 & 0 & 0 & 1 & 0 & 0 & 0 & 0 & 0 & 0 & 0 & 0 \\
X_{17} & 1 & 0 & 0 & 0 & 1 & 1 & 0 & 0 & 1 & 0 & 1 & 1 & 0 & 0 & 0 & 0 & 0 & 0 & 0 & 0 & 0 \\
X_{21} & 0 & 1 & 1 & 1 & 0 & 0 & 1 & 1 & 0 & 1 & 0 & 0 & 0 & 0 & 0 & 0 & 1 & 1 & 1 & 0 & 0 \\
X_{23}^{1} & 0 & 1 & 0 & 0 & 1 & 0 & 1 & 0 & 1 & 0 & 0 & 0 & 0 & 0 & 0 & 0 & 0 & 1 & 0 & 0 & 0 \\
X_{23}^{2} & 0 & 1 & 0 & 0 & 1 & 0 & 1 & 0 & 0 & 1 & 0 & 0 & 1 & 0 & 0 & 0 & 0 & 1 & 0 & 0 & 0 \\
X_{34}^{1} & 1 & 0 & 0 & 1 & 0 & 0 & 0 & 0 & 1 & 0 & 1 & 0 & 0 & 0 & 1 & 0 & 1 & 0 & 0 & 1 & 0 \\
X_{34}^{2} & 1 & 0 & 0 & 1 & 0 & 0 & 0 & 0 & 0 & 1 & 1 & 0 & 1 & 0 & 1 & 0 & 1 & 0 & 0 & 1 & 0 \\
X_{36} & 1 & 0 & 1 & 1 & 0 & 1 & 0 & 1 & 0 & 0 & 0 & 0 & 0 & 0 & 0 & 0 & 1 & 0 & 1 & 0 & 0 \\
X_{42} & 0 & 0 & 1 & 0 & 0 & 1 & 0 & 1 & 0 & 0 & 0 & 1 & 0 & 1 & 0 & 1 & 0 & 0 & 1 & 0 & 1 \\
X_{45}^{1} & 0 & 1 & 1 & 0 & 0 & 0 & 0 & 0 & 1 & 0 & 0 & 0 & 0 & 1 & 0 & 0 & 0 & 0 & 0 & 0 & 0 \\
X_{45}^{2} & 0 & 1 & 1 & 0 & 0 & 0 & 0 & 0 & 0 & 1 & 0 & 0 & 1 & 1 & 0 & 0 & 0 & 0 & 0 & 0 & 0 \\
X_{51} & 0 & 0 & 0 & 1 & 0 & 0 & 1 & 1 & 0 & 0 & 0 & 0 & 0 & 0 & 1 & 1 & 0 & 0 & 0 & 0 & 0 \\
X_{53} & 0 & 0 & 0 & 0 & 1 & 1 & 1 & 1 & 0 & 0 & 0 & 1 & 0 & 0 & 0 & 1 & 0 & 1 & 1 & 0 & 1 \\
X_{62} & 0 & 0 & 0 & 0 & 0 & 0 & 0 & 0 & 1 & 0 & 1 & 1 & 0 & 1 & 1 & 1 & 0 & 0 & 0 & 1 & 1 \\
X_{67} & 0 & 0 & 0 & 0 & 0 & 0 & 0 & 0 & 0 & 1 & 1 & 1 & 0 & 0 & 0 & 0 & 0 & 0 & 0 & 0 & 0 \\
X_{72} & 0 & 0 & 0 & 0 & 0 & 0 & 0 & 0 & 0 & 0 & 0 & 0 & 1 & 1 & 1 & 1 & 0 & 0 & 0 & 1 & 1 \\
X_{74} & 0 & 0 & 0 & 0 & 0 & 0 & 0 & 0 & 0 & 0 & 0 & 0 & 0 & 0 & 0 & 0 & 1 & 1 & 1 & 1 & 1
\end{array}\right)
$$


where the relations between bifundamentals and GLSM fields can be directly read off. Then we can get the total charge matrix:

$$
Q_{t}=\left(\begin{array}{ccccccccccccccccccccc}
r_{1} & r_{2} & s_{1} & r_{3} & r_{4} & s_{2} & r_{5} & s_{3} & p_{1} & p_{2} & r_{6} & s_{4} & p_{3} & r_{7} & p_{4} & r_{8} & s_{5} & s_{6} & p_{5} & r_{9} & s_{7} \\
\hline 2 & 2 & -1 & 0 & -1 & 0 & 0 & 0 & -1 & 0 & 0 & 0 & -1 & 0 & 0 & 0 & -1 & 0 & 0 & 0 & 1 \\
1 & 1 & 0 & 0 & 0 & 0 & 0 & 0 & -1 & 0 & 0 & 0 & -1 & 0 & 0 & 0 & -1 & 0 & 0 & 1 & 0 \\
1 & 1 & -1 & 0 & -1 & 0 & 0 & 0 & 0 & 0 & 0 & 0 & 0 & 0 & 0 & 0 & -1 & 0 & 1 & 0 & 0 \\
1 & 0 & 0 & 0 & -1 & 0 & 0 & 0 & 0 & 0 & 0 & 0 & 0 & 0 & 0 & 0 & -1 & 1 & 0 & 0 & 0 \\
2 & 2 & -1 & -1 & -1 & 0 & 0 & 0 & -1 & 0 & 0 & 0 & -1 & 0 & 0 & 1 & 0 & 0 & 0 & 0 & 0 \\
1 & 1 & 0 & -1 & 0 & 0 & 0 & 0 & -1 & 0 & 0 & 0 & -1 & 0 & 1 & 0 & 0 & 0 & 0 & 0 & 0 \\
1 & 1 & -1 & 0 & 0 & 0 & 0 & 0 & -1 & 0 & 0 & 0 & -1 & 1 & 0 & 0 & 0 & 0 & 0 & 0 & 0 \\
1 & 2 & -1 & 0 & -1 & 0 & 0 & 0 & -1 & -1 & 0 & 1 & 0 & 0 & 0 & 0 & 0 & 0 & 0 & 0 & 0 \\
0 & 1 & 0 & 0 & 0 & 0 & 0 & 0 & -1 & -1 & 1 & 0 & 0 & 0 & 0 & 0 & 0 & 0 & 0 & 0 & 0 \\
1 & 1 & -1 & -1 & -1 & 0 & 0 & 1 & 0 & 0 & 0 & 0 & 0 & 0 & 0 & 0 & 0 & 0 & 0 & 0 & 0 \\
1 & 0 & 0 & -1 & -1 & 0 & 1 & 0 & 0 & 0 & 0 & 0 & 0 & 0 & 0 & 0 & 0 & 0 & 0 & 0 & 0 \\
0 & 1 & -1 & 0 & -1 & 1 & 0 & 0 & 0 & 0 & 0 & 0 & 0 & 0 & 0 & 0 & 0 & 0 & 0 & 0 & 0 \\
\hline 2 & 0 & 0 & -1 & 0 & 0 & 0 & 0 & 0 & 1 & 0 & 0 & -1 & 0 & 0 & 0 & -1 & 0 & 0 & 0 & 0 \\
2 & 1 & 0 & -1 & 0 & 0 & 0 & 0 & -1 & -1 & 0 & 0 & 0 & 0 & 0 & 0 & 0 & 0 & 0 & 0 & 0 \\
2 & 1 & 0 & -2 & -1 & 0 & 0 & 0 & 0 & 0 & 0 & 0 & 0 & 0 & 0 & 0 & 0 & 0 & 0 & 0 & 0 \\
1 & 0 & -1 & -1 & 0 & 0 & 0 & 0 & 0 & 0 & 0 & 0 & 0 & 0 & 0 & 0 & 1 & 0 & 0 & 0 & 0 \\
0 & 0 & 0 & -1 & 1 & 0 & 0 & 0 & 0 & 0 & 0 & 0 & 0 & 0 & 0 & 0 & 0 & 0 & 0 & 0 & 0 \\
0 & -2 & 1 & -1 & 0 & 0 & 0 & 0 & 1 & 0 & 0 & 0 & 1 & 0 & 0 & 0 & 0 & 0 & 0 & 0 & 0
\end{array}\right)
$$

with kernel

$$
G_{t}=\left(\begin{array}{ccccccccccccccccccccc}
r_{1} & r_{2} & s_{1} & r_{3} & r_{4} & s_{2} & r_{5} & s_{3} & p_{1} & p_{2} & r_{6} & s_{4} & p_{3} & r_{7} & p_{4} & r_{8} & s_{5} & s_{6} & p_{5} & r_{9} & s_{7} \\
\hline 0 & 0 & 1 & 0 & 0 & 1 & 0 & 1 & -1 & 1 & 0 & 1 & 0 & 0 & -1 & 0 & 1 & 1 & 2 & 0 & 1 \\
1 & 1 & 0 & 1 & 1 & 0 & 1 & 0 & 3 & -1 & 1 & 0 & 0 & 1 & 2 & 1 & 0 & 0 & -1 & 1 & 0 \\
1 & 1 & 1 & 1 & 1 & 1 & 1 & 1 & 1 & 1 & 1 & 1 & 1 & 1 & 1 & 1 & 1 & 1 & 1 & 1 & 1
\end{array}\right)
$$

From $G_{t}$, we can get the GLSM fields associated to each point as shown in (5.2), where

$$
r=\left\{r_{1}, \ldots, r_{9}\right\}, s=\left\{s_{1}, \ldots, s_{7}\right\} .
$$

From $Q_{t}$ (and $\left.Q_{F}\right)$, the mesonic symmetry reads $\mathrm{U}(1)^{2} \times \mathrm{U}(1)_{\mathrm{R}}$ and the baryonic symmetry reads $\mathrm{U}(1)_{\mathrm{h}}^{4} \times \mathrm{U}(1)^{2}$, where the subscripts " $\mathrm{R}$ " and " $\mathrm{h}$ " indicate $\mathrm{R}$ - and hidden symmetries respectively.

The Hilbert series of the toric cone is

$$
\begin{aligned}
H S= & \frac{1}{\left(1-t_{2}\right)\left(1-\frac{t_{1} t_{2}}{t_{3}}\right)\left(1-\frac{t_{3}^{2}}{t_{1} t_{2}^{2}}\right)}+\frac{1}{\left(1-\frac{1}{t_{2}}\right)\left(1-\frac{t_{1}}{t_{2} t_{3}}\right)\left(1-\frac{t_{2}^{2} t_{3}^{2}}{t_{1}}\right)} \\
& +\frac{1}{\left(1-\frac{1}{t_{1}}\right)\left(1-t_{2}\right)\left(1-\frac{t_{1} t_{3}}{t_{2}}\right)}+\frac{1}{\left(1-t_{1} t_{3}\right)\left(1-t_{2} t_{3}\right)\left(1-\frac{1}{t_{1} t_{2} t_{3}}\right)} \\
& +\frac{1}{\left(1-t_{1}\right)\left(1-\frac{1}{t_{2}}\right)\left(1-\frac{t_{2} t_{3}}{t_{1}}\right)}+\frac{1}{\left(1-\frac{1}{t_{1}}\right)\left(1-\frac{1}{t_{2}}\right)\left(1-t_{1} t_{2} t_{3}\right)} \\
& +\frac{1}{\left(1-t_{1}\right)\left(1-t_{2}\right)\left(1-\frac{t_{3}}{t_{1} t_{2}}\right)} .
\end{aligned}
$$

The volume function is then

$$
V=-\frac{b_{1}^{2}-2 b_{1}\left(4 b_{2}+15\right)+4\left(b_{2}^{2}-6 b_{2}-45\right)}{\left(b_{1}+3\right)\left(b_{2}+3\right)\left(b_{1}-b_{2}+3\right)\left(b_{1}+2 b_{2}-6\right)\left(b_{1}-2\left(b_{2}+3\right)\right)} .
$$

Minimizing $V$ yields $V_{\min }=0.172260$ at $b_{1}=0.746501, b_{2}=-0.198279$. Thus, $a_{\max }=$ 1.451295. Together with the superconformal conditions, we can solve for the R-charges of 
the bifundamentals. Then the R-charges of GLSM fields should satisfy

$$
\begin{aligned}
& \left(3.75 p_{2}+1.875 p_{4}+9.375 p_{5}\right) p_{3}^{2}+\left(3.75 p_{2}^{2}+7.5 p_{4} p_{2}+1.875 p_{5} p_{2}-7.5 p_{2}+1.875 p_{4}^{2}\right. \\
& \left.+9.375 p_{5}^{2}-3.75 p_{4}+11.25 p_{4} p_{5}-18.75 p_{5}\right) p_{3}=-3.75 p_{4} p_{2}^{2}-7.5 p_{5} p_{2}^{2}-3.75 p_{4}^{2} p_{2} \\
& -7.5 p_{5}^{2} p_{2}+7.5 p_{4} p_{2}-11.25 p_{4} p_{5} p_{2}+15 p_{5} p_{2}-5.625 p_{4} p_{5}^{2}-5.625 p_{4}^{2} p_{5}+11.25 p_{4} p_{5}-3.2251
\end{aligned}
$$

constrained by $\sum_{i=1}^{5} p_{i}=2$ and $0<p_{i}<2$, with others vanishing.

\subsection{Polytope 26: $X^{3,1}$}

The polytope is

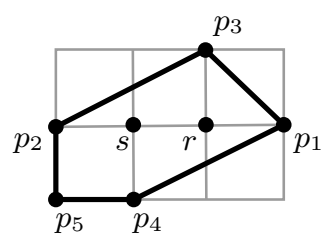

The brane tiling and the corrresponding quiver are
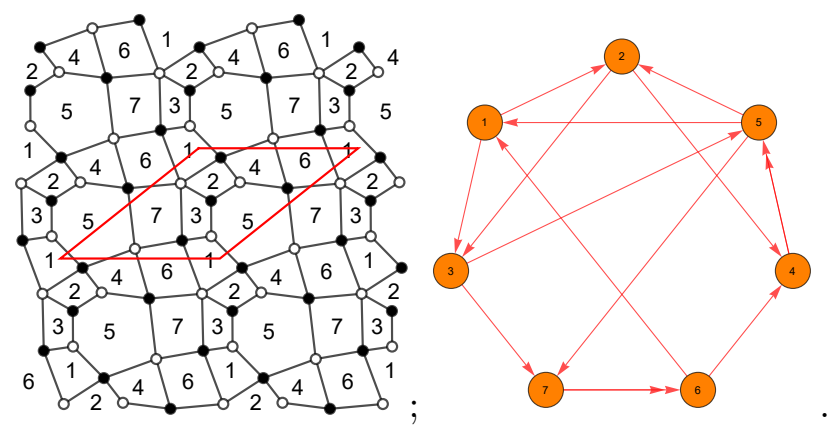

The superpotential is

$$
\begin{aligned}
W= & X_{61} X_{12} X_{23} X_{37} X_{76}^{1}+X_{35} X_{51} X_{13}+X_{24} X_{45}^{2} X_{52}+X_{57} X_{76}^{2} X_{64} X_{45}^{1} \\
& -X_{23} X_{35} X_{52}-X_{51} X_{12} X_{24} X_{45}^{1}-X_{45}^{2} X_{57} X_{76}^{1} X_{64}-X_{76}^{2} X_{61} X_{13} X_{37}
\end{aligned}
$$

The perfect matching matrix is

$$
P=\left(\begin{array}{c|cccccccccccccccccccc} 
& s_{1} & s_{2} & r_{1} & r_{2} & r_{3} & s_{3} & r_{4} & p_{1} & r_{5} & s_{4} & s_{5} & s_{6} & p_{2} & r_{6} & s_{7} & p_{3} & p_{4} & p_{5} & r_{7} & s_{8} \\
\hline X_{12} & 0 & 0 & 0 & 0 & 1 & 0 & 0 & 0 & 0 & 0 & 0 & 1 & 0 & 0 & 0 & 0 & 1 & 0 & 0 & 0 \\
X_{13} & 1 & 0 & 1 & 0 & 1 & 0 & 0 & 0 & 0 & 1 & 0 & 1 & 0 & 0 & 0 & 1 & 0 & 0 & 0 & 0 \\
X_{23} & 1 & 0 & 1 & 0 & 0 & 0 & 0 & 0 & 0 & 1 & 0 & 0 & 0 & 0 & 0 & 0 & 0 & 1 & 0 & 0 \\
X_{24} & 0 & 0 & 1 & 1 & 0 & 0 & 0 & 1 & 0 & 1 & 1 & 0 & 0 & 1 & 0 & 0 & 0 & 0 & 1 & 0 \\
X_{35} & 0 & 1 & 0 & 1 & 0 & 0 & 1 & 1 & 0 & 0 & 1 & 0 & 0 & 1 & 0 & 0 & 1 & 0 & 1 & 0 \\
X_{37} & 0 & 1 & 0 & 1 & 0 & 1 & 0 & 0 & 0 & 0 & 1 & 0 & 1 & 0 & 0 & 0 & 0 & 0 & 0 & 0 \\
X_{45}^{1} & 1 & 1 & 0 & 0 & 0 & 0 & 1 & 0 & 0 & 0 & 0 & 0 & 0 & 0 & 0 & 1 & 0 & 0 & 0 & 0 \\
X_{45}^{2} & 1 & 1 & 0 & 0 & 0 & 0 & 1 & 0 & 0 & 0 & 0 & 0 & 0 & 0 & 0 & 0 & 1 & 1 & 0 & 0 \\
X_{51} & 0 & 0 & 0 & 0 & 0 & 1 & 0 & 0 & 1 & 0 & 0 & 0 & 1 & 0 & 1 & 0 & 0 & 1 & 0 & 1 \\
X_{52} & 0 & 0 & 0 & 0 & 1 & 1 & 0 & 0 & 1 & 0 & 0 & 1 & 1 & 0 & 1 & 1 & 0 & 0 & 0 & 1 \\
X_{57} & 0 & 0 & 1 & 1 & 1 & 1 & 0 & 1 & 1 & 0 & 0 & 0 & 0 & 0 & 0 & 0 & 0 & 0 & 0 & 0 \\
X_{61} & 0 & 0 & 0 & 0 & 0 & 0 & 1 & 1 & 1 & 0 & 0 & 0 & 0 & 1 & 1 & 0 & 0 & 0 & 0 & 0 \\
X_{64} & 0 & 0 & 0 & 0 & 0 & 0 & 0 & 0 & 0 & 1 & 1 & 1 & 1 & 1 & 1 & 0 & 0 & 0 & 0 & 0 \\
X_{76}^{1} & 0 & 0 & 0 & 0 & 0 & 0 & 0 & 0 & 0 & 0 & 0 & 0 & 0 & 0 & 0 & 1 & 0 & 0 & 1 & 1 \\
X_{76}^{2} & 0 & 0 & 0 & 0 & 0 & 0 & 0 & 0 & 0 & 0 & 0 & 0 & 0 & 0 & 0 & 0 & 1 & 1 & 1 & 1
\end{array}\right),
$$


where the relations between bifundamentals and GLSM fields can be directly read off. Then we can get the total charge matrix:

$$
Q_{t}=\left(\begin{array}{cccccccccccccccccccc}
s_{1} & s_{2} & r_{1} & r_{2} & r_{3} & s_{3} & r_{4} & p_{1} & r_{5} & s_{4} & s_{5} & s_{6} & p_{2} & r_{6} & s_{7} & p_{3} & p_{4} & p_{5} & r_{7} & s_{8} \\
\hline 0 & 1 & 0 & 0 & 1 & -1 & 0 & 0 & 0 & 0 & 0 & 0 & 0 & 0 & 0 & -1 & -1 & 0 & 0 & 1 \\
1 & 0 & -1 & 0 & 1 & 0 & 0 & 0 & 0 & 0 & 0 & 0 & 0 & 0 & 0 & -1 & -1 & 0 & 1 & 0 \\
-1 & 1 & 0 & 0 & 1 & -1 & 0 & 0 & 0 & 0 & 0 & 0 & 0 & 0 & 0 & 0 & -1 & 1 & 0 & 0 \\
0 & 1 & 1 & 0 & 0 & -1 & -1 & 0 & 0 & -1 & 0 & 0 & 0 & 0 & 1 & 0 & 0 & 0 & 0 & 0 \\
1 & 0 & 0 & 0 & 0 & 0 & -1 & 0 & 0 & -1 & 0 & 0 & 0 & 1 & 0 & 0 & 0 & 0 & 0 & 0 \\
0 & 0 & 1 & 0 & 0 & -1 & 0 & 0 & 0 & -1 & 0 & 0 & 1 & 0 & 0 & 0 & 0 & 0 & 0 & 0 \\
0 & 0 & 1 & 0 & -1 & 0 & 0 & 0 & 0 & -1 & 0 & 1 & 0 & 0 & 0 & 0 & 0 & 0 & 0 & 0 \\
1 & -1 & 0 & 0 & 0 & 0 & 0 & 0 & 0 & -1 & 1 & 0 & 0 & 0 & 0 & 0 & 0 & 0 & 0 & 0 \\
0 & 1 & 0 & 0 & 0 & -1 & -1 & 0 & 1 & 0 & 0 & 0 & 0 & 0 & 0 & 0 & 0 & 0 & 0 & 0 \\
1 & 0 & -1 & 0 & 0 & 0 & -1 & 1 & 0 & 0 & 0 & 0 & 0 & 0 & 0 & 0 & 0 & 0 & 0 & 0 \\
1 & -1 & -1 & 1 & 0 & 0 & 0 & 0 & 0 & 0 & 0 & 0 & 0 & 0 & 0 & 0 & 0 & 0 & 0 & 0 \\
\hline 0 & 2 & 0 & 0 & 2 & -1 & -1 & 0 & 0 & 0 & 0 & 0 & 0 & 0 & 0 & -1 & -1 & 0 & 0 & 0 \\
0 & 1 & 1 & 0 & 0 & -1 & -2 & 0 & 0 & -1 & 0 & 0 & 0 & 0 & 0 & 1 & 1 & 0 & 0 & 0 \\
0 & 2 & 0 & 0 & 1 & -2 & -1 & 0 & 0 & 0 & 0 & 0 & 0 & 0 & 0 & 0 & 0 & 0 & 0 & 0 \\
-1 & 1 & 0 & 0 & 1 & -1 & -1 & 0 & 0 & 1 & 0 & 0 & 0 & 0 & 0 & 0 & 0 & 0 & 0 & 0 \\
1 & 0 & 0 & 0 & 1 & -1 & -1 & 0 & 0 & 0 & 0 & 0 & 0 & 0 & 0 & 0 & 0 & 0 & 0 & 0 \\
0 & 1 & -1 & 0 & 2 & -1 & -1 & 0 & 0 & 0 & 0 & 0 & 0 & 0 & 0 & 0 & 0 & 0 & 0 & 0
\end{array}\right)
$$

with kernel

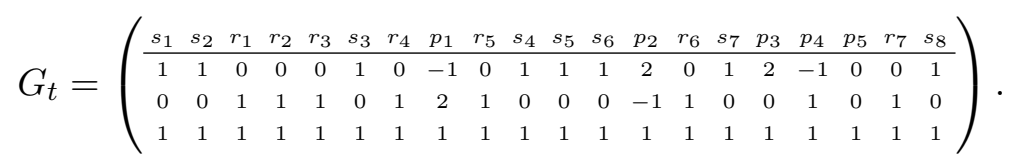

From $G_{t}$, we can get the GLSM fields associated to each point as shown in (5.12), where

$$
r=\left\{r_{1}, \ldots, r_{7}\right\}, \quad s=\left\{s_{1}, \ldots, s_{8}\right\} .
$$

From $Q_{t}$ (and $Q_{F}$ ), the mesonic symmetry reads $\mathrm{U}(1)^{2} \times \mathrm{U}(1)_{\mathrm{R}}$ and the baryonic symmetry reads $\mathrm{U}(1)_{\mathrm{h}}^{4} \times \mathrm{U}(1)^{2}$, where the subscripts " $\mathrm{R}$ " and "h" indicate $\mathrm{R}$ - and hidden symmetries respectively.

The Hilbert series of the toric cone is

$$
\begin{aligned}
H S= & \frac{1}{\left(1-\frac{1}{t_{2}}\right)\left(1-\frac{t_{1}}{t_{2} t_{3}}\right)\left(1-\frac{t_{2}^{2} t_{3}^{2}}{t_{1}}\right)}+\frac{1}{\left(1-t_{2}\right)\left(1-\frac{t_{2}}{t_{1}}\right)\left(1-\frac{t_{1} t_{3}}{t_{2}^{2}}\right)} \\
& +\frac{1}{\left(1-t_{2}\right)\left(1-\frac{t_{1}}{t_{3}}\right)\left(1-\frac{t_{3}^{2}}{t_{1} t_{2}}\right)}+\frac{1}{\left(1-t_{1} t_{3}\right)\left(1-t_{2} t_{3}\right)\left(1-\frac{1}{t_{1} t_{2} t_{3}}\right)} \\
& +\frac{1}{\left(1-t_{1}\right)\left(1-\frac{1}{t_{2}}\right)\left(1-\frac{t_{2} t_{3}}{t_{1}}\right)}+\frac{1}{\left(1-\frac{1}{t_{1}}\right)\left(1-\frac{1}{t_{2}}\right)\left(1-t_{1} t_{2} t_{3}\right)} \\
& +\frac{1}{\left(1-t_{2}\right)\left(1-\frac{t_{1}}{t_{2}}\right)\left(1-\frac{t_{3}}{t_{1}}\right)} .
\end{aligned}
$$

The volume function is then

$$
V=-\frac{b_{1}{ }^{2}-4 b_{1}\left(b_{2}+3\right)+4 b_{2}{ }^{2}-30 b_{2}-207}{\left(b_{1}+3\right)\left(b_{2}+3\right)\left(b_{1}-2 b_{2}+3\right)\left(b_{1}+b_{2}-6\right)\left(b_{1}-2\left(b_{2}+3\right)\right)} .
$$

Minimizing $V$ yields $V_{\min }=0.178752$ at $b_{1}=1.119414, b_{2}=-0.211974$. Thus, $a_{\max }=$ 1.398586. Together with the superconformal conditions, we can solve for the R-charges of 
the bifundamentals. Then the R-charges of GLSM fields should satisfy

$$
\begin{aligned}
& \left(1.26563 p_{2}+1.26563 p_{3}+0.421875 p_{5}\right) p_{4}^{2}+\left(1.26563 p_{2}^{2}+2.53125 p_{3} p_{2}+2.53125 p_{5} p_{2}\right. \\
& \left.-2.53125 p_{2}+1.26563 p_{3}^{2}+0.421875 p_{5}^{2}-2.53125 p_{3}+2.53125 p_{3} p_{5}-0.84375 p_{5}\right) p_{4} \\
& =-1.26563 p_{3} p_{2}^{2}-1.26563 p_{5} p_{2}^{2}-1.26563 p_{3}^{2} p_{2}-1.26563 p_{5}^{2} p_{2}+2.53125 p_{3} p_{2}-3.375 p_{3} p_{5} p_{2} \\
& \quad+2.53125 p_{5} p_{2}-1.6875 p_{3} p_{5}^{2}-1.6875 p_{3}^{2} p_{5}+3.375 p_{3} p_{5}-0.699293
\end{aligned}
$$

constrained by $\sum_{i=1}^{5} p_{i}=2$ and $0<p_{i}<2$, with others vanishing.

\subsection{Polytope 27: $\mathrm{PdP}_{4 c}(2)$}

The polytope is ${ }^{15}$

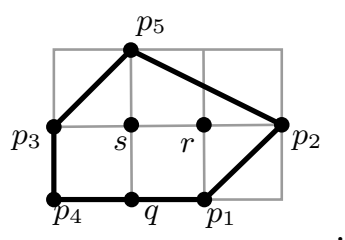

The brane tiling and the corrresponding quiver are
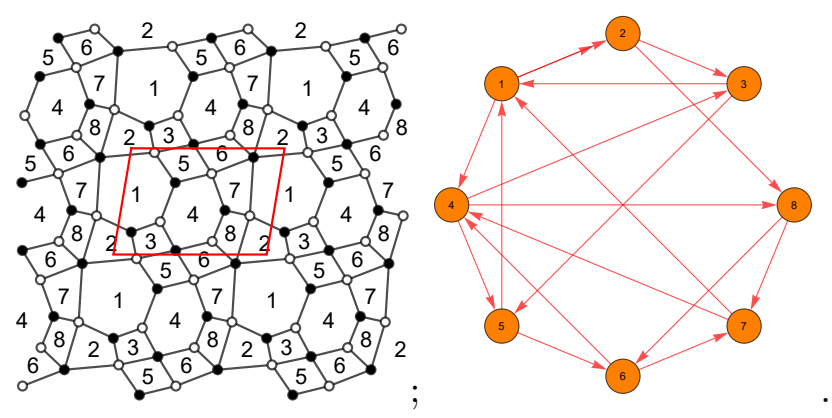

The superpotential is

$$
\begin{aligned}
W= & X_{23} X_{35} X_{51} X_{12}^{1}+X_{14} X_{43} X_{31}+X_{56} X_{67} X_{74} X_{45}+X_{48} X_{86} X_{64} \\
& +X_{71} X_{12}^{2} X_{28} X_{87}-X_{12}^{2} X_{23} X_{31}-X_{43} X_{35} X_{56} X_{64}-X_{51} X_{14} X_{45} \\
& -X_{86} X_{67} X_{71} X_{12}^{1} X_{28}-X_{74} X_{48} X_{87} .
\end{aligned}
$$

\footnotetext{
${ }^{15}$ For pseudo del Pezzos [37], our nomenclature follows the spirit of [13, 25, 26]. Hence, the labelling of $\mathrm{PdP}_{4}$ starts from $c$ in this paper. Moreover, by $\mathrm{PdP}_{n}(m)$, we mean that this comes from $\mathrm{dP}_{m}$ blown up at $(n-m)$ generic points where $m$ is chosen to be the largest possible number.
} 
The perfect matching matrix is

$$
P=\left(\begin{array}{c|cccccccccccccccccccccccccc} 
& s_{1} & s_{2} & s_{3} & r_{1} & r_{2} & s_{4} & p_{1} & q_{1} & r_{3} & r_{4} & p_{2} & r_{5} & s_{5} & s_{6} & p_{3} & r_{6} & s_{7} & q_{2} & p_{4} & p_{5} & r_{7} & s_{8} & s_{9} & s_{10} & r_{8} & s_{11} \\
\hline X_{12}^{1} & 0 & 1 & 0 & 1 & 0 & 0 & 0 & 0 & 0 & 0 & 0 & 0 & 1 & 0 & 0 & 0 & 0 & 0 & 0 & 1 & 0 & 0 & 0 & 0 & 0 & 0 \\
X_{12}^{2} & 0 & 1 & 0 & 1 & 0 & 0 & 1 & 1 & 0 & 0 & 0 & 0 & 1 & 0 & 0 & 0 & 0 & 1 & 1 & 0 & 0 & 0 & 0 & 0 & 0 & 0 \\
X_{14} & 0 & 0 & 0 & 1 & 1 & 0 & 1 & 0 & 0 & 0 & 1 & 0 & 1 & 1 & 0 & 1 & 0 & 1 & 0 & 0 & 1 & 0 & 0 & 0 & 1 & 0 \\
X_{23} & 0 & 0 & 1 & 0 & 1 & 0 & 0 & 0 & 0 & 1 & 1 & 0 & 0 & 1 & 0 & 1 & 0 & 0 & 0 & 0 & 1 & 0 & 0 & 1 & 1 & 0 \\
X_{28} & 1 & 0 & 1 & 0 & 1 & 1 & 0 & 0 & 0 & 0 & 0 & 0 & 0 & 1 & 1 & 0 & 0 & 0 & 0 & 0 & 0 & 0 & 0 & 0 & 0 & 0 \\
X_{31} & 1 & 0 & 0 & 0 & 0 & 1 & 0 & 0 & 1 & 0 & 0 & 1 & 0 & 0 & 1 & 0 & 1 & 0 & 0 & 1 & 0 & 1 & 1 & 0 & 0 & 1 \\
X_{35} & 1 & 0 & 0 & 0 & 0 & 0 & 1 & 0 & 1 & 0 & 0 & 0 & 0 & 0 & 0 & 0 & 0 & 1 & 0 & 0 & 0 & 0 & 1 & 0 & 0 & 0 \\
X_{43} & 0 & 1 & 1 & 0 & 0 & 0 & 0 & 1 & 0 & 1 & 0 & 0 & 0 & 0 & 0 & 0 & 0 & 0 & 1 & 0 & 0 & 0 & 0 & 1 & 0 & 0 \\
X_{45} & 1 & 1 & 1 & 0 & 0 & 0 & 0 & 0 & 1 & 1 & 0 & 0 & 0 & 0 & 0 & 0 & 0 & 0 & 0 & 1 & 0 & 0 & 1 & 1 & 0 & 0 \\
X_{48} & 1 & 1 & 1 & 1 & 1 & 1 & 1 & 1 & 1 & 1 & 1 & 1 & 0 & 0 & 0 & 0 & 0 & 0 & 0 & 0 & 0 & 0 & 0 & 0 & 0 & 0 \\
X_{51} & 0 & 0 & 0 & 0 & 0 & 1 & 0 & 1 & 0 & 0 & 0 & 1 & 0 & 0 & 1 & 0 & 1 & 0 & 1 & 0 & 0 & 1 & 0 & 0 & 0 & 1 \\
X_{56} & 0 & 0 & 0 & 1 & 1 & 1 & 0 & 0 & 0 & 0 & 1 & 1 & 0 & 0 & 0 & 0 & 0 & 0 & 0 & 0 & 0 & 0 & 0 & 0 & 1 & 1 \\
X_{64} & 0 & 0 & 0 & 0 & 0 & 0 & 0 & 0 & 0 & 0 & 0 & 0 & 1 & 1 & 1 & 1 & 1 & 0 & 0 & 1 & 1 & 1 & 0 & 0 & 0 & 0 \\
X_{67} & 0 & 0 & 0 & 0 & 0 & 0 & 1 & 1 & 0 & 0 & 0 & 0 & 0 & 0 & 0 & 0 & 0 & 0 & 0 & 0 & 1 & 1 & 0 & 0 & 0 & 0 \\
X_{71} & 0 & 0 & 0 & 0 & 0 & 0 & 0 & 0 & 1 & 1 & 1 & 1 & 0 & 0 & 0 & 1 & 1 & 0 & 0 & 0 & 0 & 0 & 0 & 0 & 0 & 0 \\
X_{74} & 0 & 0 & 0 & 0 & 0 & 0 & 0 & 0 & 0 & 0 & 0 & 0 & 1 & 1 & 1 & 1 & 1 & 1 & 1 & 0 & 0 & 0 & 0 & 0 & 0 & 0 \\
X_{86} & 0 & 0 & 0 & 0 & 0 & 0 & 0 & 0 & 0 & 0 & 0 & 0 & 0 & 0 & 0 & 0 & 0 & 1 & 1 & 0 & 0 & 0 & 1 & 1 & 1 \\
X_{87} & 0 & 0 & 0 & 0 & 0 & 0 & 0 & 0 & 0 & 0 & 0 & 0 & 0 & 0 & 0 & 0 & 0 & 0 & 0 & 1 & 1 & 1 & 1 & 1 & 1 & 1
\end{array}\right)
$$

where the relations between bifundamentals and GLSM fields can be directly read off. Then we can get the total charge matrix:

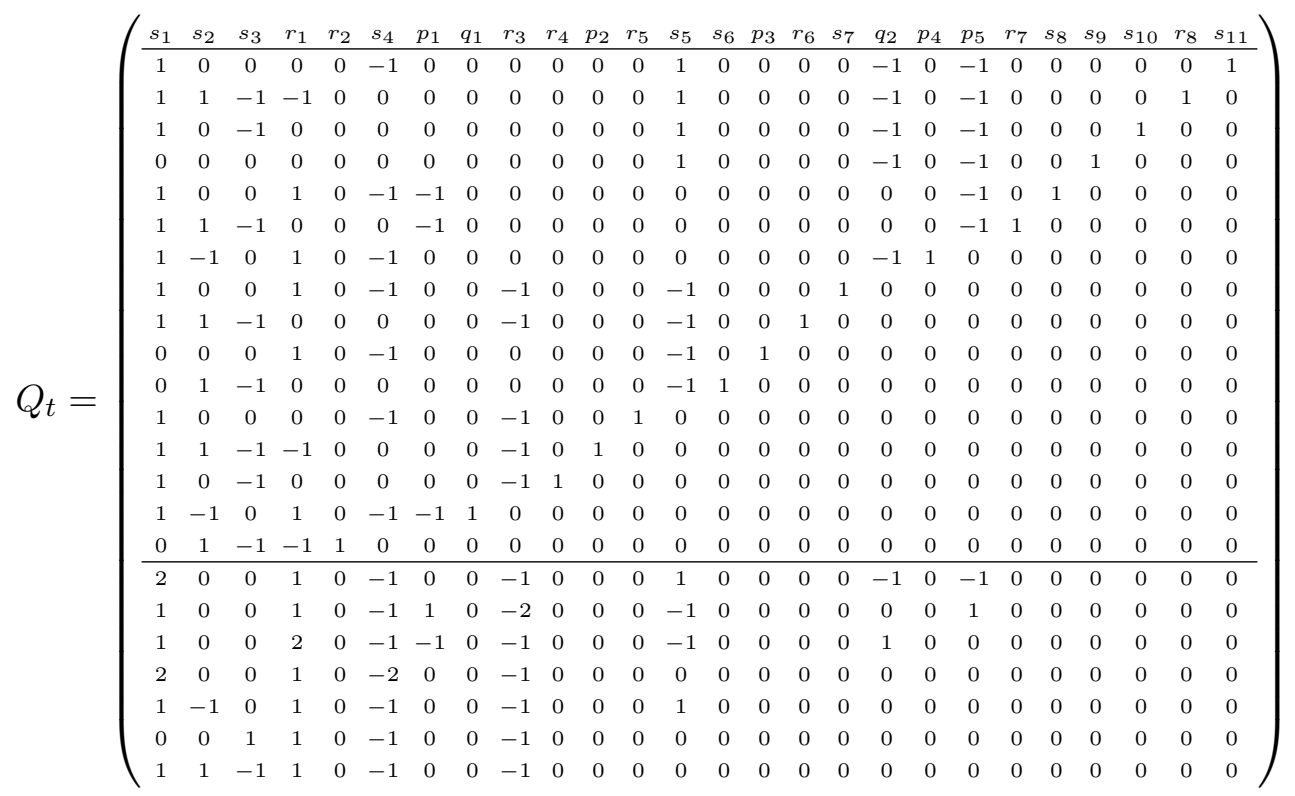

with kernel

$$
G_{t}=\left(\begin{array}{cccccccccccccccccccccccccc}
s_{1} & s_{2} & s_{3} & r_{1} & r_{2} & s_{4} & p_{1} & q_{1} & r_{3} & r_{4} & p_{2} & r_{5} & s_{5} & s_{6} & p_{3} & r_{6} & s_{7} & q_{2} & p_{4} & p_{5} & r_{7} & s_{8} & s_{9} & s_{10} & r_{8} & s_{11} \\
\hline 1 & 1 & 1 & 0 & 0 & 1 & 1 & 2 & 0 & 0 & -1 & 0 & 1 & 1 & 2 & 0 & 1 & 2 & 3 & 0 & 0 & 1 & 1 & 1 & 0 & 1 \\
0 & 0 & 0 & 1 & 1 & 0 & 1 & 0 & 1 & 1 & 2 & 1 & 0 & 0 & -1 & 1 & 0 & 0 & -1 & 0 & 1 & 0 & 0 & 0 & 1 & 0 \\
1 & 1 & 1 & 1 & 1 & 1 & 1 & 1 & 1 & 1 & 1 & 1 & 1 & 1 & 1 & 1 & 1 & 1 & 1 & 1 & 1 & 1 & 1 & 1 & 1 & 1
\end{array}\right) \text {. }
$$

From $G_{t}$, we can get the GLSM fields associated to each point as shown in (5.22), where

$$
q=\left\{q_{1}, q_{2}\right\}, r=\left\{r_{1}, \ldots, r_{8}\right\}, s=\left\{s_{1}, \ldots, s_{11}\right\} .
$$

From $Q_{t}$ (and $Q_{F}$ ), the mesonic symmetry reads $\mathrm{U}(1)^{2} \times \mathrm{U}(1)_{\mathrm{R}}$ and the baryonic symmetry reads $\mathrm{U}(1)_{\mathrm{h}}^{4} \times \mathrm{U}(1)^{3}$, where the subscripts " $\mathrm{R}$ " and " $\mathrm{h}$ " indicate $\mathrm{R}$ - and hidden symmetries respectively. 
The Hilbert series of the toric cone is

$$
\begin{aligned}
H S= & \frac{1}{\left(1-t_{2}\right)\left(1-\frac{t_{1} t_{2}}{t_{3}}\right)\left(1-\frac{t_{3}^{2}}{t_{1} t_{2}^{2}}\right)}+\frac{1}{\left(1-\frac{1}{t_{2}}\right)\left(1-\frac{t_{1}}{t_{3}}\right)\left(1-\frac{t_{2} t_{3}^{2}}{t_{1}}\right)} \\
& +\frac{1}{\left(1-t_{1}\right)\left(1-t_{2}\right)\left(1-\frac{t_{3}}{t_{1} t_{2}}\right)}+\frac{1}{\left(1-\frac{1}{t_{1}}\right)\left(1-t_{2}\right)\left(1-\frac{t_{1} t_{3}}{t_{2}}\right)} \\
& +\frac{1}{\left(1-\frac{1}{t_{1}}\right)\left(1-\frac{t_{1}}{t_{2}}\right)\left(1-t_{2} t_{3}\right)}+\frac{1}{\left(1-\frac{t_{3}}{t_{1}}\right)\left(1-t_{2} t_{3}\right)\left(1-\frac{t_{1}}{t_{2} t_{3}}\right)} \\
& +\frac{1}{\left(1-t_{1}\right)\left(1-\frac{1}{t_{2}}\right)\left(1-\frac{t_{2} t_{3}}{t_{1}}\right)}+\frac{1}{\left(1-\frac{1}{t_{2}}\right)\left(1-\frac{t_{2}}{t_{1}}\right)\left(1-t_{1} t_{3}\right)} .
\end{aligned}
$$

The volume function is then

$$
V=-\frac{2 b_{1}^{2}-4 b_{1}\left(b_{2}+6\right)+2 b_{2}^{2}-3 b_{2}-171}{\left(b_{1}+3\right)\left(b_{2}+3\right)\left(b_{1}-b_{2}-6\right)\left(b_{1}-b_{2}+3\right)\left(b_{1}+2 b_{2}-6\right)} .
$$

Minimizing $V$ yields $V_{\min }=0.155420$ at $b_{1}=0.933751, b_{2}=-0.449691$. Thus, $a_{\max }=$ 1.608545. Together with the superconformal conditions, we can solve for the R-charges of the bifundamentals. Then the R-charges of GLSM fields should satisfy

$$
\begin{gathered}
\left(0.50625 p_{2}+0.50625 p_{3}+0.675 p_{4}\right) p_{5}^{2}+\left(0.50625 p_{2}^{2}+1.0125 p_{3} p_{2}+0.675 p_{4} p_{2}-1.0125 p_{2}\right. \\
\left.+0.50625 p_{3}^{2}+0.675 p_{4}^{2}-1.0125 p_{3}+1.35 p_{3} p_{4}-1.35 p_{4}\right) p_{5}=-0.50625 p_{3} p_{2}^{2}-0.3375 p_{4} p_{2}^{2} \\
\quad-0.50625 p_{3}^{2} p_{2}-0.3375 p_{4}^{2} p_{2}+1.0125 p_{3} p_{2}-0.675 p_{3} p_{4} p_{2}+0.675 p_{4} p_{2}-0.3375 p_{3} p_{4}^{2} \\
-0.3375 p_{3}^{2} p_{4}+0.675 p_{3} p_{4}-0.321709
\end{gathered}
$$

constrained by $\sum_{i=1}^{5} p_{i}=2$ and $0<p_{i}<2$, with others vanishing.

\subsection{Polytope 28: $\mathrm{PdP}_{4 d}(2)$}

The polytope is

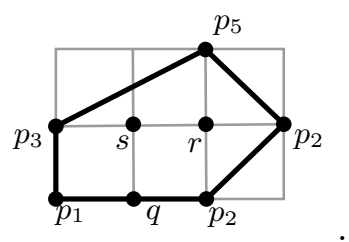

The brane tiling and the corrresponding quiver are
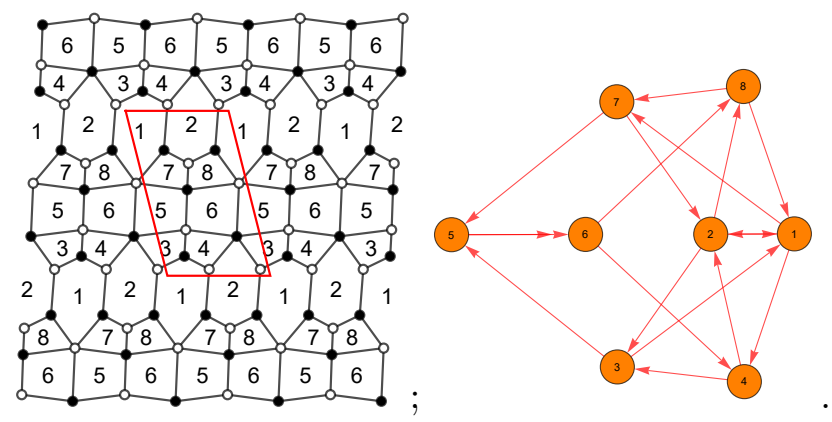
The superpotential is

$$
\begin{aligned}
W= & X_{23} X_{31} X_{12}+X_{14} X_{42} X_{21}+X_{35} X_{56}^{1} X_{64} X_{43}+X_{68} X_{81} X_{17} X_{75} X_{56}^{2} \\
& +X_{72} X_{28} X_{87}-X_{31} X_{14} X_{43}-X_{42} X_{23} X_{35} X_{56}^{2} X_{64}-X_{56}^{1} X_{68} X_{87} X_{75} \\
& -X_{17} X_{72} X_{21}-X_{28} X_{81} X_{12} .
\end{aligned}
$$

The perfect matching matrix is

$$
P=\left(\begin{array}{c|ccccccccccccccccccccccccc} 
& q_{1} & p_{1} & s_{1} & r_{1} & r_{2} & s_{2} & r_{3} & p_{2} & r_{4} & r_{5} & s_{3} & s_{4} & p_{3} & r_{6} & s_{5} & s_{6} & p_{4} & q_{2} & r_{7} & s_{7} & p_{5} & s_{8} & r_{8} & r_{9} & s_{9} \\
\hline X_{12} & 0 & 0 & 0 & 0 & 0 & 0 & 1 & 1 & 1 & 1 & 0 & 0 & 0 & 1 & 1 & 1 & 0 & 0 & 1 & 1 & 1 & 1 & 0 & 0 & 0 \\
X_{14} & 1 & 0 & 0 & 0 & 0 & 0 & 1 & 1 & 0 & 1 & 0 & 0 & 0 & 1 & 0 & 1 & 1 & 0 & 1 & 0 & 0 & 1 & 0 & 0 & 0 \\
X_{17} & 1 & 1 & 0 & 0 & 0 & 0 & 0 & 0 & 0 & 0 & 0 & 0 & 0 & 0 & 0 & 0 & 0 & 0 & 1 & 1 & 0 & 1 & 0 & 0 & 0 \\
X_{21} & 0 & 0 & 1 & 1 & 1 & 1 & 0 & 0 & 0 & 0 & 1 & 1 & 1 & 0 & 0 & 0 & 0 & 0 & 0 & 0 & 1 & 0 & 1 & 1 & 1 \\
X_{23} & 1 & 0 & 0 & 0 & 1 & 0 & 0 & 0 & 0 & 0 & 0 & 1 & 0 & 0 & 0 & 0 & 1 & 0 & 0 & 0 & 0 & 0 & 0 & 1 & 0 \\
X_{28} & 1 & 1 & 1 & 1 & 1 & 1 & 0 & 0 & 0 & 0 & 1 & 1 & 1 & 0 & 0 & 0 & 0 & 0 & 0 & 0 & 0 & 0 & 0 & 0 & 0 \\
X_{31} & 0 & 1 & 1 & 1 & 0 & 1 & 0 & 0 & 0 & 0 & 1 & 0 & 1 & 0 & 0 & 0 & 0 & 1 & 0 & 0 & 0 & 0 & 1 & 0 & 1 \\
X_{35} & 0 & 0 & 0 & 1 & 0 & 0 & 0 & 1 & 0 & 0 & 1 & 0 & 0 & 1 & 0 & 0 & 0 & 0 & 1 & 0 & 0 & 0 & 1 & 0 & 0 \\
X_{42} & 0 & 1 & 0 & 0 & 0 & 0 & 0 & 0 & 1 & 0 & 0 & 0 & 0 & 0 & 1 & 0 & 0 & 1 & 0 & 1 & 0 & 0 & 0 & 0 & 0 \\
X_{43} & 0 & 0 & 0 & 0 & 1 & 0 & 0 & 0 & 1 & 0 & 0 & 1 & 0 & 0 & 1 & 0 & 0 & 0 & 0 & 1 & 1 & 0 & 0 & 1 & 0 \\
X_{56}^{1} & 1 & 1 & 1 & 0 & 0 & 0 & 1 & 0 & 0 & 0 & 0 & 0 & 0 & 0 & 0 & 0 & 1 & 1 & 0 & 0 & 0 & 0 & 0 & 0 & 0 \\
X_{56}^{2} & 0 & 0 & 1 & 0 & 0 & 0 & 1 & 0 & 0 & 0 & 0 & 0 & 0 & 0 & 0 & 0 & 0 & 0 & 0 & 0 & 1 & 0 & 0 & 0 & 0 \\
X_{64} & 0 & 0 & 0 & 0 & 0 & 1 & 0 & 0 & 0 & 1 & 0 & 0 & 1 & 0 & 0 & 1 & 0 & 0 & 0 & 0 & 0 & 1 & 0 & 0 & 1 \\
X_{68} & 0 & 0 & 0 & 1 & 1 & 1 & 0 & 1 & 1 & 1 & 0 & 0 & 0 & 0 & 0 & 0 & 0 & 0 & 0 & 0 & 0 & 0 & 0 & 0 & 0 \\
X_{72} & 0 & 0 & 0 & 0 & 0 & 0 & 1 & 1 & 1 & 1 & 0 & 0 & 0 & 1 & 1 & 1 & 1 & 1 & 0 & 0 & 0 & 0 & 0 & 0 & 0 \\
X_{75} & 0 & 0 & 0 & 0 & 0 & 0 & 0 & 0 & 0 & 0 & 1 & 1 & 1 & 1 & 1 & 1 & 0 & 0 & 0 & 0 & 0 & 0 & 0 & 0 & 0 \\
X_{81} & 0 & 0 & 0 & 0 & 0 & 0 & 0 & 0 & 0 & 0 & 0 & 0 & 0 & 0 & 0 & 0 & 1 & 1 & 0 & 0 & 0 & 0 & 1 & 1 \\
X_{87} & 0 & 0 & 0 & 0 & 0 & 0 & 0 & 0 & 0 & 0 & 0 & 0 & 0 & 0 & 0 & 0 & 0 & 0 & 1 & 1 & 1 & 1 & 1 & 1 & 1
\end{array}\right)
$$

where the relations between bifundamentals and GLSM fields can be directly read off. Then we can get the total charge matrix:

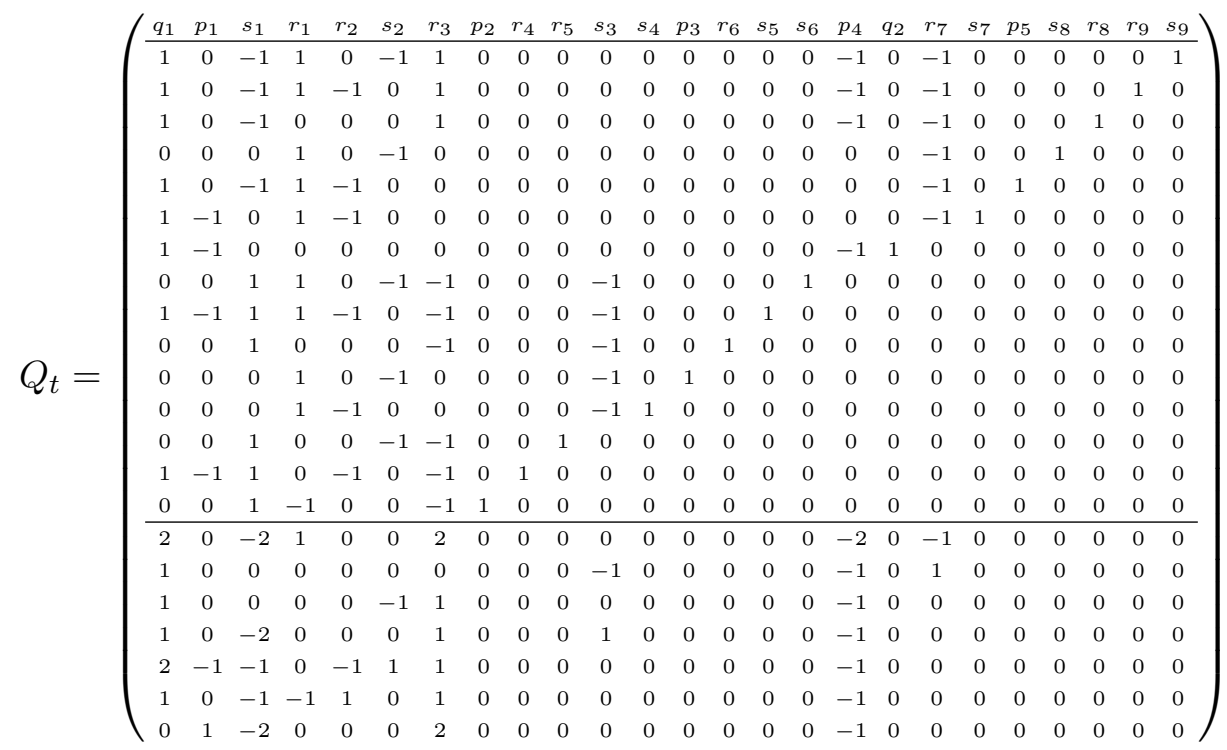

with kernel

$$
G_{t}=\left(\begin{array}{ccccccccccccccccccccccccc}
q_{1} & p_{1} & s_{1} & r_{1} & r_{2} & s_{2} & r_{3} & p_{2} & r_{4} & r_{5} & s_{3} & s_{4} & p_{3} & r_{6} & s_{5} & s_{6} & p_{4} & q_{2} & r_{7} & s_{7} & p_{5} & s_{8} & r_{8} & r_{9} & s_{9} \\
\hline 1 & 2 & 1 & 0 & 0 & 1 & 0 & -1 & 0 & 0 & 1 & 1 & 2 & 0 & 1 & 1 & 0 & 1 & 0 & 1 & 0 & 1 & 0 & 0 & 1 \\
1 & 0 & 0 & 1 & 1 & 0 & 1 & 2 & 1 & 1 & 0 & 0 & -1 & 1 & 0 & 0 & 2 & 1 & 1 & 0 & 0 & 0 & 1 & 1 & 0 \\
1 & 1 & 1 & 1 & 1 & 1 & 1 & 1 & 1 & 1 & 1 & 1 & 1 & 1 & 1 & 1 & 1 & 1 & 1 & 1 & 1 & 1 & 1 & 1 & 1
\end{array}\right) .
$$

From $G_{t}$, we can get the GLSM fields associated to each point as shown in (5.32), where

$$
q=\left\{q_{1}, q_{2}\right\}, \quad r=\left\{r_{1}, \ldots, r_{9}\right\}, \quad s=\left\{s_{1}, \ldots, s_{9}\right\} .
$$


From $Q_{t}$ (and $Q_{F}$ ), the mesonic symmetry reads $\mathrm{U}(1)^{2} \times \mathrm{U}(1)_{\mathrm{R}}$ and the baryonic symmetry reads $\mathrm{U}(1)_{\mathrm{h}}^{4} \times \mathrm{U}(1)^{3}$, where the subscripts " $\mathrm{R}$ " and "h" indicate $\mathrm{R}$ - and hidden symmetries respectively.

The Hilbert series of the toric cone is

$$
\begin{aligned}
H S= & \frac{1}{\left(1-t_{2}\right)\left(1-\frac{t_{2}}{t_{1}}\right)\left(1-\frac{t_{1} t_{3}}{t_{2}^{2}}\right)}+\frac{1}{\left(1-t_{2}\right)\left(1-\frac{t_{1}}{t_{3}}\right)\left(1-\frac{t_{3}^{2}}{t_{1} t_{2}}\right)} \\
& +\frac{1}{\left(1-\frac{1}{t_{2}}\right)\left(1-\frac{t_{1}}{t_{3}}\right)\left(1-\frac{t_{2} t_{3}^{2}}{t_{1}}\right)}+\frac{1}{\left(1-\frac{1}{t_{2}}\right)\left(1-\frac{t_{2}}{t_{1}}\right)\left(1-t_{1} t_{3}\right)} \\
& +\frac{1}{\left(1-\frac{1}{t_{1}}\right)\left(1-\frac{t_{1}}{t_{2}}\right)\left(1-t_{2} t_{3}\right)}+\frac{1}{\left(1-t_{1}\right)\left(1-\frac{1}{t_{2}}\right)\left(1-\frac{t_{2} t_{3}}{t_{1}}\right)} \\
& +\frac{1}{\left(1-t_{2}\right)\left(1-\frac{t_{1}}{t_{2}}\right)\left(1-\frac{t_{3}}{t_{1}}\right)}+\frac{t_{\left.t_{2} t_{3}\right)\left(1-\frac{t_{1}}{t_{2} t_{3}}\right)}}{\left(1-\frac{t_{3}}{t_{1}}\right)\left(1-t_{1}\right.}
\end{aligned}
$$

The volume function is then

$$
V=-\frac{2\left(b_{1}^{2}-b_{1}\left(b_{2}+3\right)+b_{2}^{2}-3 b_{2}-99\right)}{\left(b_{1}+3\right)\left(b_{2}+3\right)\left(b_{1}-2 b_{2}+3\right)\left(b_{1}-b_{2}-6\right)\left(b_{1}+b_{2}-6\right)}
$$

Minimizing $V$ yields $V_{\min }=0.158756$ at $b_{1}=1.266149, b_{2}=-0.467702$. Thus, $a_{\max }=$ 1.574744. Together with the superconformal conditions, we can solve for the R-charges of the bifundamentals. Then the R-charges of GLSM fields should satisfy

$$
\begin{aligned}
& \left(1.26563 p_{3}+843750 . p_{4}+1.6875 p_{5}\right) p_{2}^{2}+\left(1.26563 p_{3}^{2}+1.6875 p_{4} p_{3}+2.53125 p_{5} p_{3}\right. \\
& \left.-2.53125 p_{3}+0.84375 p_{4}^{2}+1.6875 p_{5}^{2}-1.6875 p_{4}+3.375 p_{4} p_{5}-3.375 p_{5}\right) p_{2} \\
& =-0.84375 p_{4} p_{3}^{2}-0.84375 p_{5} p_{3}^{2}-0.84375 p_{4}^{2} p_{3}-0.84375 p_{5}^{2} p_{3}+1.6875 p_{4} p_{3}-1.6875 p_{4} p_{5} p_{3} \\
& \quad+1.6875 p_{5} p_{3}-1.6875 p_{4} p_{5}^{2}-1.6875 p_{4}^{2} p_{5}+3.375 p_{4} p_{5}-0.787372
\end{aligned}
$$

constrained by $\sum_{i=1}^{5} p_{i}=2$ and $0<p_{i}<2$, with others vanishing.

\subsection{Polytope 29: $\mathrm{PdP}_{5 b}(2)$}

The polytope is ${ }^{16}$

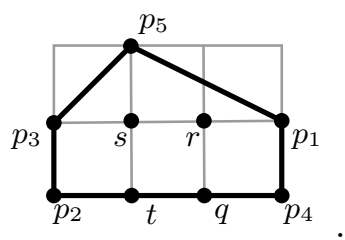

\footnotetext{
${ }^{16} \operatorname{In}[13,25,26]$, there is only one $\mathrm{PdP}_{5}$ (hence without a further alphabet subscript). We will regard it as $5 a$, and this polygon is $5 b$.
} 
The brane tiling and the corrresponding quiver are
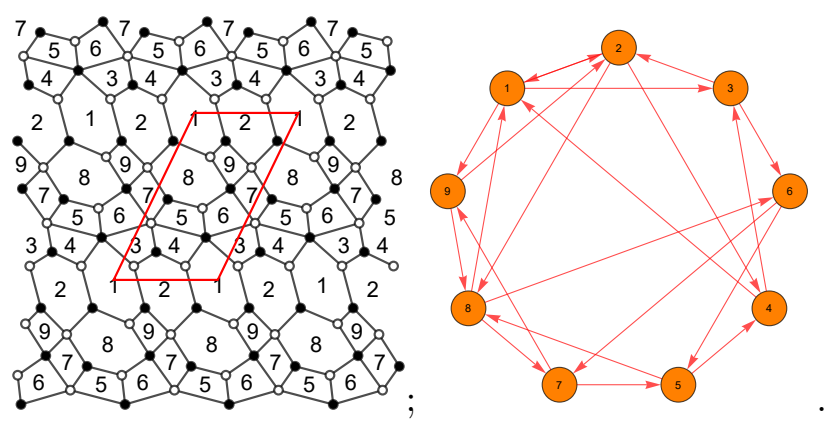

The superpotential is

$$
\begin{aligned}
W= & X_{13} X_{32} X_{21}+X_{24} X_{41} X_{12}+X_{58} X_{86} X_{65}+X_{67} X_{75} X_{54} X_{43} X_{36} \\
& +X_{81} X_{19} X_{98}+X_{92} X_{28} X_{87} X_{79}-X_{41} X_{13} X_{36} X_{65} X_{54}-X_{32} X_{24} X_{43} \\
& -X_{75} X_{58} X_{87}-X_{86} X_{67} X_{79} X_{98}-X_{28} X_{81} X_{12}-X_{19} X_{92} X_{21}
\end{aligned}
$$

The number of perfect matchings is $c=33$, which leads to gigantic $P, Q_{t}$ and $G_{t}$. Hence, we will not list them here. The GLSM fields associated to each point are shown in (5.32), where

$$
q=\left\{q_{1}, \ldots, q_{3}\right\}, \quad r=\left\{r_{1}, \ldots, r_{10}\right\}, \quad s=\left\{s_{1}, \ldots, s_{12}\right\}, \quad t=\left\{t_{1}, \ldots, t_{3}\right\} .
$$

The mesonic symmetry reads $\mathrm{U}(1)^{2} \times \mathrm{U}(1)_{\mathrm{R}}$ and the baryonic symmetry reads $\mathrm{U}(1)_{\mathrm{h}}^{4} \times \mathrm{U}(1)^{4}$, where the subscripts "R" and "h" indicate R- and hidden symmetries respectively.

The Hilbert series of the toric cone is

$$
\begin{aligned}
H S= & \frac{1}{\left(1-t_{2}\right)\left(1-\frac{t_{1} t_{2}}{t_{3}}\right)\left(1-\frac{t_{3}^{2}}{t_{1} t_{2}^{2}}\right)}+\frac{1}{\left(1-\frac{t_{3}^{2}}{t_{1}}\right)\left(1-t_{2} t_{3}\right)\left(1-\frac{t_{1}}{t_{2} t_{3}^{2}}\right)} \\
& +\frac{1}{\left(1-\frac{1}{t_{2}}\right)\left(1-\frac{t_{1}}{t_{3}}\right)\left(1-\frac{t_{2} t_{3}^{2}}{t_{1}}\right)}+\frac{1}{\left(1-t_{1}\right)\left(1-t_{2}\right)\left(1-\frac{t_{3}}{t_{1} t_{2}}\right)} \\
& +\frac{1}{\left(1-\frac{1}{t_{1}}\right)\left(1-t_{2}\right)\left(1-\frac{t_{1} t_{3}}{t_{2}}\right)}+\frac{1}{\left(1-\frac{1}{t_{1}}\right)\left(1-\frac{t_{1}}{t_{2}}\right)\left(1-t_{2} t_{3}\right)} \\
& +\frac{1}{\left(1-\frac{t_{3}}{t_{1}}\right)\left(1-t_{2} t_{3}\right)\left(1-\frac{t_{1}}{t_{2} t_{3}}\right)}+\frac{1}{\left(1-t_{1}\right)\left(1-\frac{1}{t_{2}}\right)\left(1-\frac{t_{2} t_{3}}{t_{1}}\right)} \\
& +\frac{1}{\left(1-\frac{1}{t_{2}}\right)\left(1-\frac{t_{2}}{t_{1}}\right)\left(1-t_{1} t_{3}\right)} .
\end{aligned}
$$

The volume function is then

$$
V=-\frac{3\left(b_{1}{ }^{2}-6 b_{1}+6\left(b_{2}-9\right)\right)}{\left(b_{1}-6\right)\left(b_{1}+3\right)\left(b_{2}+3\right)\left(b_{1}-b_{2}+3\right)\left(b_{1}+2 b_{2}-6\right)} .
$$

Minimizing $V$ yields $V_{\min }=0.136079$ at $b_{1}=1.322699, b_{2}=-0.700670$. Thus, $a_{\max }=$ 1.837168. Together with the superconformal conditions, we can solve for the R-charges of 
the bifundamentals. Then the R-charges of GLSM fields should satisfy

$$
\begin{aligned}
& \left(1.26563 p_{2}+1.26563 p_{4}+1.26563 p_{5}\right) p_{3}^{2}+\left(1.26563 p_{2}^{2}+2.53125 p_{4} p_{2}+4.21875 p_{5} p_{2}\right. \\
& \left.-2.53125 p_{2}+1.26563 p_{4}^{2}+1.26563 p_{5}^{2}-2.53125 p_{4}+1.6875 p_{4} p_{5}-2.53125 p_{5}\right) p_{3} \\
& =-1.26563 p_{4} p_{2}^{2}-2.10938 p_{5} p_{2}^{2}-1.26563 p_{4}^{2} p_{2}-2.10938 p_{5}^{2} p_{2}+2.53125 p_{4} p_{2} \\
& \quad-1.6875 p_{4} p_{5} p_{2}+4.21875 p_{5} p_{2}-0.84375 p_{4} p_{5}^{2}-0.84375 p_{4}^{2} p_{5}+1.6875 p_{4} p_{5}-0.918584
\end{aligned}
$$

constrained by $\sum_{i=1}^{5} p_{i}=2$ and $0<p_{i}<2$, with others vanishing.

\subsection{Polytope 30: $\mathrm{PdP}_{6 a}(2)$}

The polytope is

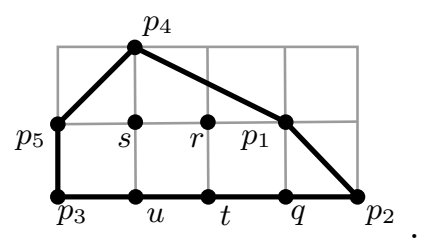

The brane tiling and the corrresponding quiver are
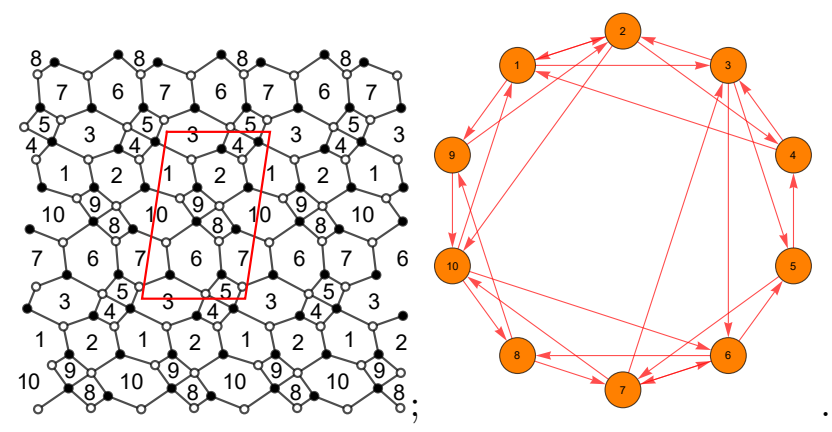

The superpotential is

$$
\begin{aligned}
W= & X_{13} X_{32} X_{21}+X_{24} X_{41} X_{12}+X_{36} X_{65} X_{54} X_{43}+X_{57} X_{73} X_{35} \\
& +X_{68} X_{87} X_{76}+X_{7,10} X_{10,6} X_{67}+X_{10,1} X_{19} X_{9,10}+X_{92} X_{2,10} X_{10,8} X_{89} \\
& -X_{2,10} X_{10,1} X_{12}-X_{41} X_{13} X_{35} X_{54}-X_{32} X_{24} X_{43}-X_{65} X_{57} X_{76} \\
& -X_{73} X_{36} X_{67}-X_{87} X_{7,10} X_{10,8}-X_{10,6} X_{68} X_{89} X_{9,10}-X_{19} X_{92} X_{21} .
\end{aligned}
$$

The number of perfect matchings is $c=53$, which leads to gigantic $P, Q_{t}$ and $G_{t}$. Hence, we will not list them here. The GLSM fields associated to each point are shown in (5.49), where

$$
\begin{aligned}
q & =\left\{q_{1}, \ldots, q_{3}\right\}, & r & =\left\{r_{1}, \ldots, r_{17}\right\}, \\
u & =\left\{u_{1}, \ldots, u_{3}\right\}, & s & =\left\{s_{1}, \ldots, s_{17}\right\} .
\end{aligned}
$$

The mesonic symmetry reads $\mathrm{U}(1)^{2} \times \mathrm{U}(1)_{\mathrm{R}}$ and the baryonic symmetry reads $\mathrm{U}(1)_{\mathrm{h}}^{4} \times \mathrm{U}(1)^{5}$, where the subscripts "R" and "h" indicate R- and hidden symmetries respectively. 
The Hilbert series of the toric cone is

$$
\begin{aligned}
H S= & \frac{1}{\left(1-t_{2}\right)\left(1-\frac{t_{1} t_{2}}{t_{3}}\right)\left(1-\frac{t_{3}^{2}}{t_{1} t_{2}^{2}}\right)}+\frac{1}{\left(1-\frac{1}{t_{2}}\right)\left(1-\frac{t_{3}^{2}}{t_{1}}\right)\left(1-\frac{t_{1} t_{2}}{t_{3}}\right)} \\
& +\frac{1}{\left(1-\frac{t_{1}}{t_{3}^{2}}\right)\left(1-t_{2} t_{3}\right)\left(1-\frac{t_{3}^{2}}{t_{1} t_{2}}\right)}+\frac{1}{\left(1-t_{1}\right)\left(1-t_{2}\right)\left(1-\frac{t_{3}}{t_{1} t_{2}}\right)} \\
& +\frac{1}{\left(1-\frac{1}{t_{1}}\right)\left(1-t_{2}\right)\left(1-\frac{t_{1} t_{3}}{t_{2}}\right)}+\frac{1}{\left(1-t_{1}\right)\left(1-\frac{1}{t_{1} t_{2}}\right)\left(1-t_{2} t_{3}\right)} \\
& +\frac{1}{\left(1-t_{1} t_{3}\right)\left(1-t_{2} t_{3}\right)\left(1-\frac{1}{t_{1} t_{2} t_{3}}\right)}+\frac{1}{\left(1-\frac{t_{1}}{t_{3}}\right)\left(1-t_{2} t_{3}\right)\left(1-\frac{t_{3}}{t_{1} t_{2}}\right)} \\
& +\frac{1}{\left(1-\frac{1}{t_{1}}\right)\left(1-\frac{1}{t_{2}}\right)\left(1-t_{1} t_{2} t_{3}\right)}+\frac{1}{\left(1-\frac{1}{t_{2}}\right)\left(1-t_{1} t_{2}\right)\left(1-\frac{t_{3}}{t_{1}}\right)} .
\end{aligned}
$$

The volume function is then

$$
V=-\frac{4 b_{1}^{2}+4 b_{1}\left(b_{2}-3\right)-2 b_{2}^{2}+39 b_{2}-153}{\left(b_{1}+3\right)\left(b_{2}+3\right)\left(b_{1}-b_{2}+3\right)\left(b_{1}+b_{2}-6\right)\left(b_{1}+2 b_{2}-6\right)} .
$$

Minimizing $V$ yields $V_{\min }=0.116367$ at $b_{1}=1.939465, b_{2}=-0.878930$. Thus, $a_{\max }=$ 2.148375. Together with the superconformal conditions, we can solve for the R-charges of the bifundamentals. Then the R-charges of GLSM fields should satisfy

$$
\begin{aligned}
& \left(2.25 p_{2}+11.25 p_{3}+6.75 p_{5}\right) p_{4}^{2}+\left(2.25 p_{2}^{2}+4.5 p_{3} p_{2}+4.5 p_{5} p_{2}-4.5 p_{2}+11.25 p_{3}^{2}\right. \\
& \left.+6.75 p_{5}^{2}-22.5 p_{3}+22.5 p_{3} p_{5}-13.5 p_{5}\right) p_{4}=-9 . p_{3} p_{2}^{2}-6.75 p_{5} p_{2}^{2}-9 p_{3}^{2} p_{2}-6.75 p_{5}^{2} p_{2} \\
& +18 p_{3} p_{2}-13.5 p_{3} p_{5} p_{2}+13.5 p_{5} p_{2}-6.75 p_{3} p_{5}^{2}-6.75 p_{3}^{2} p_{5}+13.5 p_{3} p_{5}-5.729
\end{aligned}
$$

constrained by $\sum_{i=1}^{5} p_{i}=2$ and $0<p_{i}<2$, with others vanishing.

\subsection{Polytope 31: $K^{2,5,1,4}$}

The polytope is

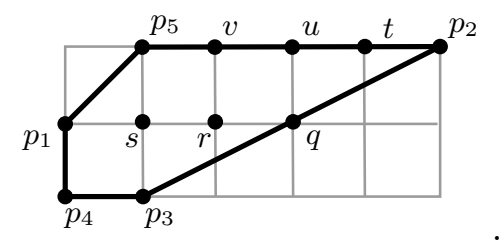

The brane tiling and the corrresponding quiver are
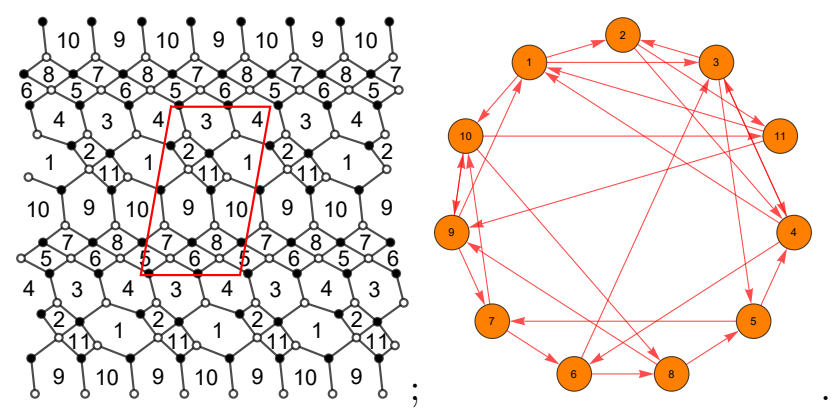
The superpotential is

$$
\begin{aligned}
W= & X_{1,3} X_{3,4} X_{4,1}+X_{2,4} X_{4,3} X_{3,2}+X_{3,5} X_{5,7} X_{7,6} X_{6,3}+X_{4,6} X_{6,8} X_{8,5} X_{5,4} \\
& +X_{8,9} X_{9,10} X_{10,8}+X_{7,10} X_{10,9} X_{9,7}+X_{9,1} X_{1,2} X_{2,11} X_{11,9}+X_{10,11} X_{11,1} X_{1,10} \\
& -X_{1,2} X_{2,4} X_{4,1}-X_{3,4} X_{4,6} X_{6,3}-X_{4,3} X_{3,5} X_{5,4}-X_{10,8} X_{8,5} X_{5,7} X_{7,10} \\
& -X_{76} X_{68} X_{89} X_{97}-X_{9,10} X_{10,11} X_{11,9}-X_{10,9} X_{9,1} X_{1,10}-X_{11,1} X_{1,3} X_{3,2} X_{2,11} .
\end{aligned}
$$

The number of perfect matchings is $c=66$, which leads to gigantic $P, Q_{t}$ and $G_{t}$. Hence, we will not list them here. The GLSM fields associated to each point are shown in (5.56), where

$$
\begin{array}{lll}
q=\left\{q_{1}, q_{2}\right\}, & r=\left\{r_{1}, \ldots, r_{25}\right\}, & s=\left\{s_{1}, \ldots, s_{20}\right\} \\
t=\left\{t_{1}, \ldots, t_{4}\right\}, & u=\left\{u_{1}, \ldots, u_{6}\right\}, & v=\left\{v_{1}, \ldots, v_{4}\right\} .
\end{array}
$$

The mesonic symmetry reads $\mathrm{U}(1)^{2} \times \mathrm{U}(1)_{\mathrm{R}}$ and the baryonic symmetry reads $\mathrm{U}(1)_{\mathrm{h}}^{4} \times \mathrm{U}(1)^{6}$, where the subscripts " $\mathrm{R}$ " and " $\mathrm{h}$ " indicate $\mathrm{R}$ - and hidden symmetries respectively.

The Hilbert series of the toric cone is

$$
\begin{aligned}
H S= & \frac{1}{\left(1-\frac{1}{t_{2}}\right)\left(1-\frac{t_{1}}{t_{2} t_{3}}\right)\left(1-\frac{t_{2}^{2} t_{3}^{2}}{t_{1}}\right)}+\frac{1}{\left(1-\frac{t_{3}}{t_{2}}\right)\left(1-\frac{t_{1}}{t_{2} t_{3}^{2}}\right)\left(1-\frac{t_{2}^{2} t_{3}^{2}}{t_{1}}\right)} \\
& +\frac{1}{\left(1-t_{2}\right)\left(1-\frac{t_{3}^{2}}{t_{1}}\right)\left(1-\frac{t_{1}}{t_{2} t_{3}}\right)}+\frac{1}{\left(1-\frac{t_{1}}{t_{3}^{2}}\right)\left(1-\frac{t_{3}}{t_{2}}\right)\left(1-\frac{t_{2} t_{3}^{2}}{t_{1}}\right)} \\
& +\frac{1}{\left(1-\frac{1}{t_{2}}\right)\left(1-\frac{t_{2}}{t_{1}}\right)\left(1-t_{1} t_{3}\right)}+\frac{1}{\left(1-t_{1}\right)\left(1-\frac{t_{2}}{t_{1}}\right)\left(1-\frac{t_{3}}{t_{2}}\right)} \\
& +\frac{1}{\left(1-\frac{1}{t_{1}}\right)\left(1-t_{2}\right)\left(1-\frac{t_{1} t_{3}}{t_{2}}\right)}+\frac{1}{\left(1-\frac{1}{t_{1}}\right)\left(1-\frac{t_{1}}{t_{2}}\right)\left(1-t_{2} t_{3}\right)} \\
& +\frac{1}{\left(1-t_{1}\right)\left(1-\frac{1}{t_{2}}\right)\left(1-\frac{t_{2} t_{3}}{t_{1}}\right)}+\frac{1}{\left(1-\frac{t_{1}}{t_{3}}\right)\left(1-\frac{t_{3}}{t_{2}}\right)\left(1-\frac{t_{2} t_{3}}{t_{1}}\right)} \\
& +\frac{\left(1-t_{2}\right)\left(1-\frac{t_{1}}{t_{2}}\right)\left(1-\frac{t_{3}}{t_{1}}\right)}{(1-}
\end{aligned}
$$

The volume function is then

$$
V=-\frac{2\left(b_{2}^{2}-3 b_{2}-36\right)-3 b_{1}\left(b_{2}+5\right)}{\left(b_{1}+3\right)\left(b_{2}-3\right)\left(b_{2}+3\right)\left(b_{1}-b_{2}+3\right)\left(b_{1}-2\left(b_{2}+3\right)\right)} .
$$

Minimizing $V$ yields $V_{\min }=0.106224$ at $b_{1}=2.907158, b_{2}=0.685037$. Thus, $a_{\max }=$ 2.353517. Together with the superconformal conditions, we can solve for the R-charges of the bifundamentals. Then the R-charges of GLSM fields should satisfy

$$
\begin{gathered}
\left(5.0625 p_{2}+0.84375 p_{4}+1.6875 p_{5}\right) p_{3}^{2}+\left(5.0625 p_{2}^{2}+8.4375 p_{4} p_{2}+3.375 p_{5} p_{2}\right. \\
\left.-10.125 p_{2}+0.84375 p_{4}^{2}+1.6875 p_{5}^{2}-1.6875 p_{4}+1.6875 p_{4} p_{5}-3.375 p_{5}\right) p_{3}=-4.21875 p_{4} p_{2}^{2} \\
\quad-3.375 p_{5} p_{2}^{2}-4.21875 p_{4}^{2} p_{2}-3.375 p_{5}^{2} p_{2}+8.4375 p_{4} p_{2}-1.6875 p_{4} p_{5} p_{2}+6.75 p_{5} p_{2} \\
\quad-0.84375 p_{4} p_{5}^{2}-0.84375 p_{4}^{2} p_{5}+1.6875 p_{4} p_{5}-2.35352
\end{gathered}
$$


constrained by $\sum_{i=1}^{5} p_{i}=2$ and $0<p_{i}<2$, with others vanishing.

\subsection{Polytope 32: $K^{2,5,1,3}$}

The polytope is

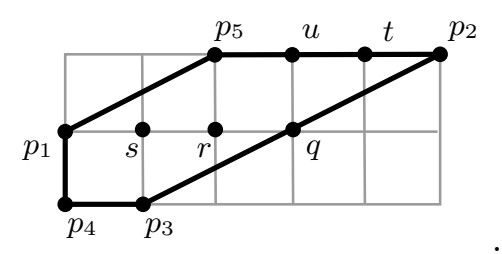

The brane tiling and the corrresponding quiver are
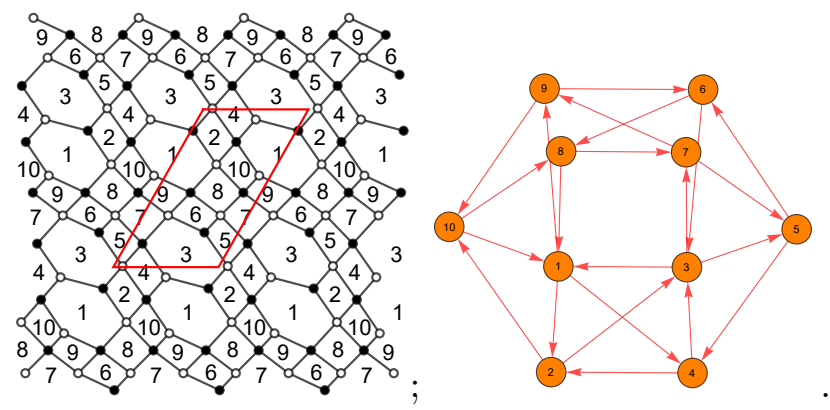

The superpotential is

$$
\begin{aligned}
W= & X_{12} X_{2,10} X_{10,8} X_{81}+X_{14} X_{43} X_{31}+X_{23} X_{35} X_{54} X_{42}+X_{56} X_{68} X_{87} X_{75} \\
& +X_{37} X_{79} X_{96} X_{63}+X_{9,10} X_{10,1} X_{19}-X_{10,1} X_{14} X_{42} X_{2,10}-X_{12} X_{23} X_{31} \\
& -X_{43} X_{37} X_{75} X_{54}-X_{35} X_{56} X_{63}-X_{96} X_{68} X_{81} X_{19}-X_{87} X_{79} X_{9,10} X_{10,8} .
\end{aligned}
$$

The number of perfect matchings is $c=46$, which leads to gigantic $P, Q_{t}$ and $G_{t}$. Hence, we will not list them here. The GLSM fields associated to each point are shown in (5.63), where

$$
\begin{aligned}
q & =\left\{q_{1}, q_{2}\right\}, & r & =\left\{r_{1}, \ldots, r_{20}\right\}, \quad s=\left\{s_{1}, \ldots, s_{13}\right\} \\
t & =\left\{t_{1}, \ldots, t_{3}\right\}, & u & =\left\{u_{1}, \ldots, u_{3}\right\} .
\end{aligned}
$$

The mesonic symmetry reads $\mathrm{U}(1)^{2} \times \mathrm{U}(1)_{\mathrm{R}}$ and the baryonic symmetry reads $\mathrm{U}(1)_{\mathrm{h}}^{4} \times \mathrm{U}(1)^{5}$, where the subscripts "R" and "h" indicate R- and hidden symmetries respectively. 
The Hilbert series of the toric cone is

$$
\begin{aligned}
H S= & \frac{1}{\left(1-\frac{1}{t_{2}}\right)\left(1-\frac{t_{1}}{t_{2} t_{3}}\right)\left(1-\frac{t_{2}^{2} t_{3}^{2}}{t_{1}}\right)}+\frac{1}{\left(1-\frac{t_{3}}{t_{2}}\right)\left(1-\frac{t_{1}}{t_{2} t_{3}^{2}}\right)\left(1-\frac{t_{2}^{2} t_{3}^{2}}{t_{1}}\right)} \\
& +\frac{1}{\left(1-t_{2}\right)\left(1-\frac{t_{2}}{t_{1}}\right)\left(1-\frac{t_{1} t_{3}}{t_{2}^{2}}\right)}+\frac{1}{\left(1-\frac{t_{3}^{2}}{t_{1}}\right)\left(1-\frac{t_{3}}{t_{2}}\right)\left(1-\frac{t_{1} t_{2}}{t_{3}^{2}}\right)} \\
& +\frac{1}{\left(1-t_{2}\right)\left(1-\frac{t_{1}}{t_{3}}\right)\left(1-\frac{t_{3}^{2}}{t_{1} t_{2}}\right)}+\frac{1}{\left(1-\frac{t_{1}}{t_{3}^{2}}\right)\left(1-\frac{t_{3}}{t_{2}}\right)\left(1-\frac{t_{2} t_{3}^{2}}{t_{1}}\right)} \\
& +\frac{1}{\left(1-t_{1} t_{3}\right)\left(1-t_{2} t_{3}\right)\left(1-\frac{1}{t_{1} t_{2} t_{3}}\right)}+\frac{1}{\left(1-t_{1}\right)\left(1-\frac{1}{t_{2}}\right)\left(1-\frac{t_{2} t_{3}}{t_{1}}\right)} \\
& +\frac{1}{\left(1-\frac{1}{t_{1}}\right)\left(1-\frac{1}{t_{2}}\right)\left(1-t_{1} t_{2} t_{3}\right)}+\frac{\left.1-t_{2}\right)\left(1-\frac{t_{1}}{t_{2}}\right)\left(1-\frac{t_{3}}{t_{1}}\right)}{(1-}
\end{aligned}
$$

The volume function is then

$$
V=-\frac{-2 b_{1}\left(b_{2}+6\right)+4 b_{2}^{2}-90}{\left(b_{1}+3\right)\left(b_{2}-3\right)\left(b_{2}+3\right)\left(b_{1}-2 b_{2}+3\right)\left(b_{1}-2\left(b_{2}+3\right)\right)} .
$$

Minimizing $V$ yields $V_{\min }=0.121782$ at $b_{1}=3.092671, b_{2}=0.479773$. Thus, $a_{\max }=$ 2.052849. Together with the superconformal conditions, we can solve for the R-charges of the bifundamentals. Then the R-charges of GLSM fields should satisfy

$$
\begin{aligned}
& \left(1.6875 p_{2}+0.28125 p_{4}+0.84375 p_{5}\right) p_{3}^{2}+\left(1.6875 p_{2}^{2}+2.8125 p_{4} p_{2}+1.6875 p_{5} p_{2}\right. \\
& \left.-3.375 p_{2}+0.28125 p_{4}^{2}+0.84375 p_{5}^{2}-0.5625 p_{4}+1.125 p_{4} p_{5}-1.6875 p_{5}\right) p_{3}=-1.40625 p_{4} p_{2}^{2} \\
& \quad-0.84375 p_{5} p_{2}^{2}-1.40625 p_{4}^{2} p_{2}-0.84375 p_{5}^{2} p_{2}+2.8125 p_{4} p_{2}-1.125 p_{4} p_{5} p_{2}+1.6875 p_{5} p_{2} \\
& \quad-0.5625 p_{4} p_{5}^{2}-0.5625 p_{4}^{2} p_{5}+1.125 p_{4} p_{5}-0.684283
\end{aligned}
$$

constrained by $\sum_{i=1}^{5} p_{i}=2$ and $0<p_{i}<2$, with others vanishing.

\subsection{Polytope 33: $K^{2,5,1,2}$}

The polytope is

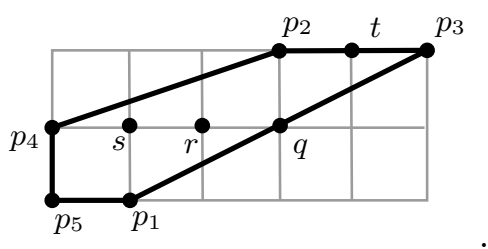


The brane tiling and the corrresponding quiver are
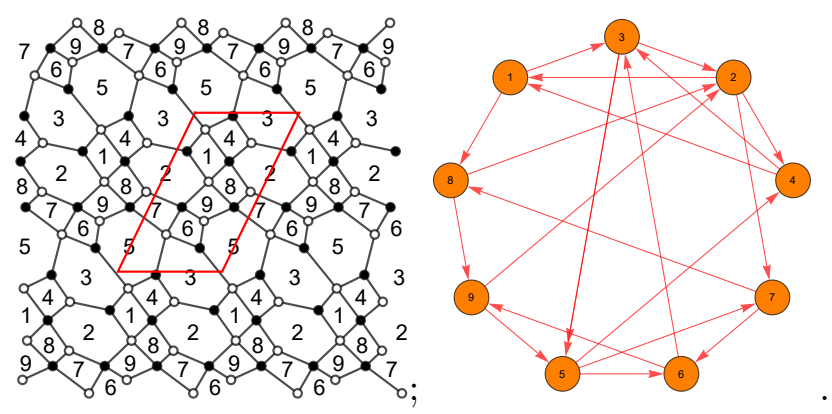

The superpotential is

$$
\begin{aligned}
W= & X_{13} X_{35}^{1} X_{54} X_{41}+X_{24} X_{43} X_{32}+X_{35}^{2} X_{57} X_{76} X_{63}+X_{56} X_{69} X_{95} \\
& +X_{78} X_{82} X_{27}+X_{89} X_{92} X_{21} X_{18}-X_{82} X_{24} X_{41} X_{18}-X_{21} X_{13} X_{32} \\
& -X_{43} X_{35}^{2} X_{54}-X_{35}^{1} X_{56} X_{63}-X_{76} X_{69} X_{92} X_{27}-X_{95} X_{57} X_{78} X_{89}
\end{aligned}
$$

The number of perfect matchings is $c=36$, which leads to gigantic $P, Q_{t}$ and $G_{t}$. Hence, we will not list them here. The GLSM fields associated to each point are shown in (5.70), where

$$
q=\left\{q_{1}, q_{2}\right\}, \quad r=\left\{r_{1}, \ldots, r_{17}\right\}, \quad s=\left\{s_{1}, \ldots, s_{10}\right\}, \quad t=\left\{t_{1}, t_{2}\right\} .
$$

The mesonic symmetry reads $\mathrm{U}(1)^{2} \times \mathrm{U}(1)_{\mathrm{R}}$ and the baryonic symmetry reads $\mathrm{U}(1)_{\mathrm{h}}^{4} \times \mathrm{U}(1)^{4}$, where the subscripts "R" and "h" indicate R- and hidden symmetries respectively.

The Hilbert series of the toric cone is

$$
\begin{aligned}
H S= & \frac{1}{\left(1-\frac{1}{t_{2}}\right)\left(1-\frac{t_{1}}{t_{2} t_{3}}\right)\left(1-\frac{t_{2}^{2} t_{3}^{2}}{t_{1}}\right)}+\frac{1}{\left(1-\frac{t_{3}}{t_{2}}\right)\left(1-\frac{t_{1}}{t_{2} t_{3}^{2}}\right)\left(1-\frac{t_{2}^{2} t_{3}^{2}}{t_{1}}\right)} \\
& +\frac{1}{\left(1-t_{2}\right)\left(1-\frac{t_{1}}{t_{2}^{2}}\right)\left(1-\frac{t_{2} t_{3}}{t_{1}}\right)}+\frac{1}{\left(1-t_{2}\right)\left(1-\frac{t_{2}^{2}}{t_{1}}\right)\left(1-\frac{t_{1} t_{3}}{t_{2}^{3}}\right)} \\
& +\frac{1}{\left(1-t_{2}\right)\left(1-\frac{t_{3}^{2}}{t_{1}}\right)\left(1-\frac{t_{1}}{t_{2} t_{3}}\right)}+\frac{1}{\left(1-\frac{t_{1}}{t_{3}^{2}}\right)\left(1-\frac{t_{3}}{t_{2}}\right)\left(1-\frac{t_{2} t_{3}^{2}}{t_{1}}\right)} \\
& +\frac{1}{\left(1-\frac{1}{t_{1}}\right)\left(1-\frac{t_{1}}{t_{2}}\right)\left(1-t_{2} t_{3}\right)}+\frac{\left.1-t_{1}\right)\left(1-\frac{1}{t_{2}}\right)\left(1-\frac{t_{2} t_{3}}{t_{1}}\right)}{\left(1-\frac{1}{t_{2}}\right)\left(1-\frac{t_{2}}{t_{1}}\right)\left(1-t_{1} t_{3}\right)} .
\end{aligned}
$$

The volume function is then

$$
V=-\frac{6\left(b_{2}^{2}+b_{2}-18\right)-b_{1}\left(b_{2}+9\right)}{\left(b_{1}+3\right)\left(b_{2}-3\right)\left(b_{2}+3\right)\left(b_{1}-3 b_{2}+3\right)\left(b_{1}-2\left(b_{2}+3\right)\right)} .
$$

Minimizing $V$ yields $V_{\min }=0.135851$ at $b_{1}=2.974853, b_{2}=0.227507$. Thus, $a_{\max }=$ 1.840251. Together with the superconformal conditions, we can solve for the R-charges of 
the bifundamentals. Then the R-charges of GLSM fields should satisfy

$$
\begin{aligned}
& \left(1.125 p_{2}+1.6875 p_{4}+0.5625 p_{5}\right) p_{3}^{2}+\left(1.125 p_{2}^{2}+3.375 p_{4} p_{2}+1.125 p_{5} p_{2}\right. \\
& \left.-2.25 p_{2}+1.6875 p_{4}^{2}+0.5625 p_{5}^{2}-3.375 p_{4}+1.125 p_{4} p_{5}-1.125 p_{5}\right) p_{3}=-1.125 p_{4} p_{2}^{2} \\
& \quad-0.5625 p_{5} p_{2}^{2}-1.125 p_{4}^{2} p_{2}-0.5625 p_{5}^{2} p_{2}+2.25 p_{4} p_{2}-1.125 p_{4} p_{5} p_{2}+1.125 p_{5} p_{2} \\
& \quad-0.28125 p_{4} p_{5}^{2}-0.28125 p_{4}^{2} p_{5}+0.5625 p_{4} p_{5}-0.613417
\end{aligned}
$$

constrained by $\sum_{i=1}^{5} p_{i}=2$ and $0<p_{i}<2$, with others vanishing.

\subsection{Polytope 34: $K^{2,5,1,1}$}

The polytope is

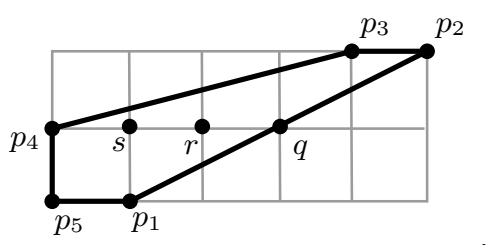

The brane tiling and the corrresponding quiver are
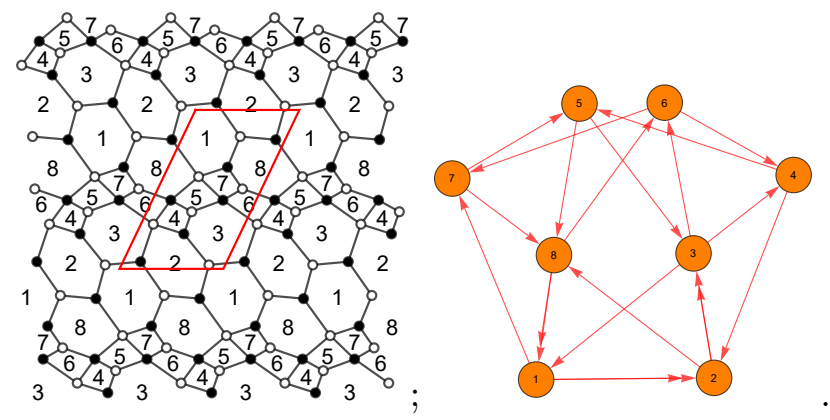

The superpotential is

$$
\begin{aligned}
W= & X_{12}^{1} X_{23}^{2} X_{31}+X_{23}^{1} X_{36} X_{64} X_{42}+X_{34} X_{45} X_{53}+X_{67} X_{78} X_{86} \\
& +X_{58} X_{81}^{2} X_{17} X_{75}+X_{81}^{1} X_{12}^{2} X_{28}-X_{31} X_{12}^{2} X_{23}^{1}-X_{23}^{2} X_{34} X_{42} \\
& -X_{64} X_{45} X_{58} X_{86}-X_{53} X_{36} X_{67} X_{75}-X_{78} X_{81}^{1} X_{17}-X_{81}^{2} X_{12}^{1} X_{28}
\end{aligned}
$$


The perfect matching matrix is

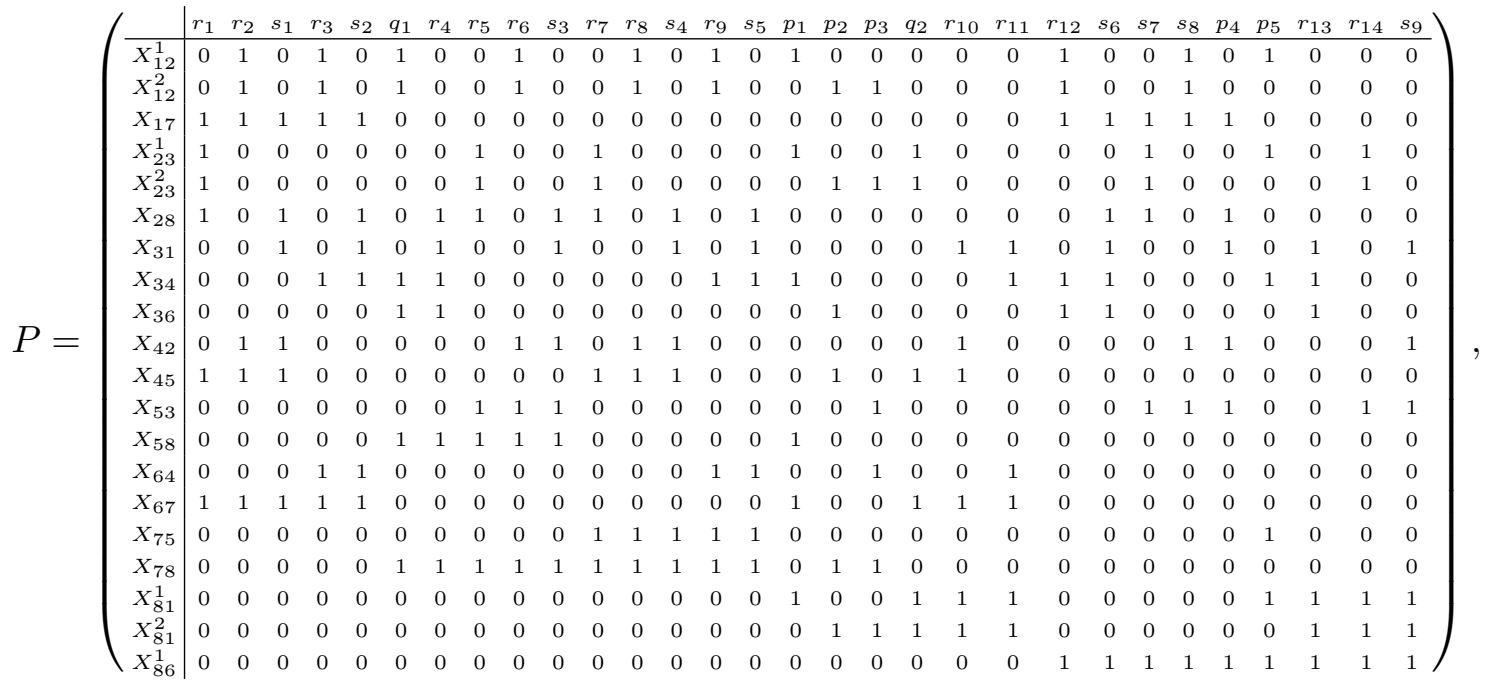

where the relations between bifundamentals and GLSM fields can be directly read off. Then we can get the total charge matrix:

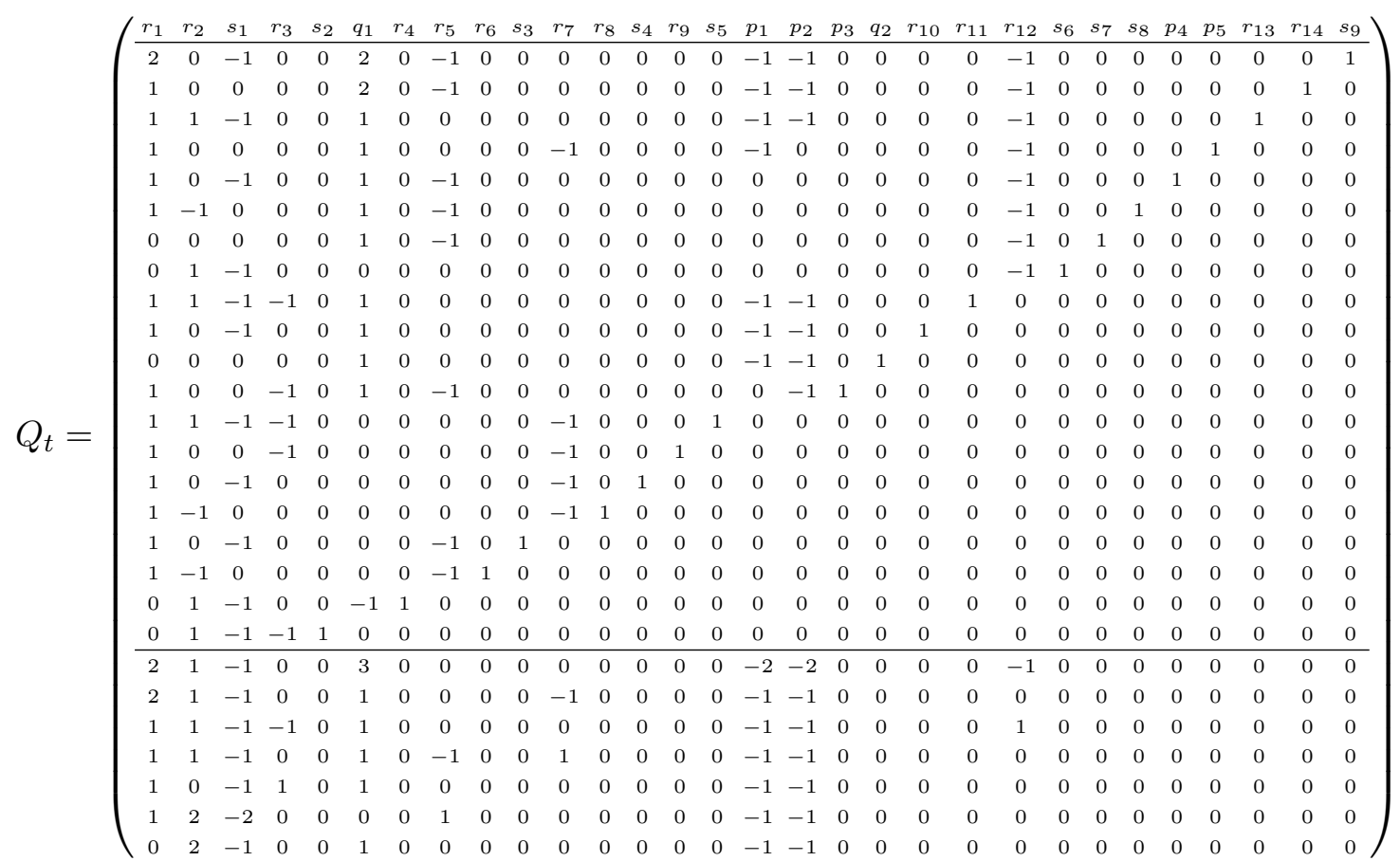

with kernel

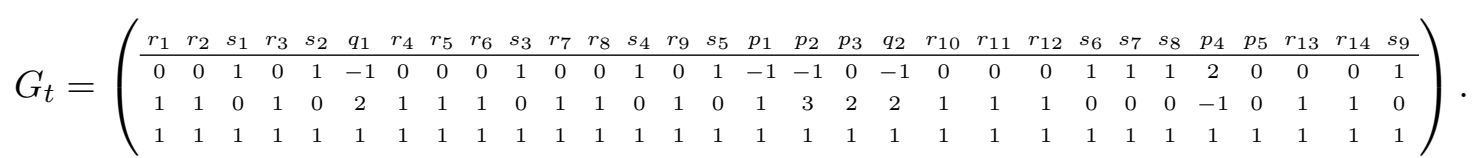

From $G_{t}$, we can get the GLSM fields associated to each point as shown in (5.77), where

$$
q=\left\{q_{1}, q_{2}\right\}, \quad r=\left\{r_{1}, \ldots, r_{14}\right\}, \quad s=\left\{s_{1}, \ldots, s_{9}\right\} .
$$


From $Q_{t}$ (and $Q_{F}$ ), the mesonic symmetry reads $\mathrm{U}(1)^{2} \times \mathrm{U}(1)_{\mathrm{R}}$ and the baryonic symmetry reads $\mathrm{U}(1)_{\mathrm{h}}^{4} \times \mathrm{U}(1)^{3}$, where the subscripts " $\mathrm{R}$ " and " $\mathrm{h}$ " indicate $\mathrm{R}$ - and hidden symmetries respectively.

The Hilbert series of the toric cone is

$$
\begin{aligned}
H S= & \frac{1}{\left(1-t_{2}\right)\left(1-\frac{t_{1}}{t_{2}^{2} t_{3}}\right)\left(1-\frac{t_{2} t_{3}^{2}}{t_{1}}\right)}+\frac{1}{\left(1-\frac{1}{t_{2}}\right)\left(1-\frac{t_{1}}{t_{2} t_{3}}\right)\left(1-\frac{t_{2}^{2} t_{3}^{2}}{t_{1}}\right)} \\
& +\frac{1}{\left(1-\frac{t_{3}}{t_{2}}\right)\left(1-\frac{t_{1}}{t_{2} t_{3}^{2}}\right)\left(1-\frac{t_{2}^{2} t_{3}^{2}}{t_{1}}\right)}+\frac{1}{\left(1-t_{2}\right)\left(1-\frac{t_{2}^{3}}{t_{1}}\right)\left(1-\frac{t_{1} t_{3}}{t_{2}^{4}}\right)} \\
& +\frac{1}{\left(1-t_{2}\right)\left(1-\frac{t_{1}}{t_{2}^{3}}\right)\left(1-\frac{t_{2}^{2} t_{3}}{t_{1}}\right)}+\frac{1}{\left(1-\frac{1}{t_{1}}\right)\left(1-\frac{t_{1}}{t_{2}}\right)\left(1-t_{2} t_{3}\right)} \\
& +\frac{1}{\left(1-t_{1}\right)\left(1-\frac{1}{t_{2}}\right)\left(1-\frac{t_{2} t_{3}}{t_{1}}\right)}+\frac{1}{\left(1-\frac{1}{t_{2}}\right)\left(1-\frac{t_{2}}{t_{1}}\right)\left(1-t_{1} t_{3}\right)} .
\end{aligned}
$$

The volume function is then

$$
V=\frac{2\left(3 b_{1}-4 b_{2}^{2}-6 b_{2}+63\right)}{\left(b_{1}+3\right)\left(b_{2}-3\right)(b-2+3)\left(b_{1}-4 b_{2}+3\right)\left(b_{1}-2\left(b_{2}+3\right)\right)} .
$$

Minimizing $V$ yields $V_{\min }=(143+19 \sqrt{57}) / 1944$ at $b_{1}=(9 \sqrt{57}-57) / 4, b_{2}=0$. Thus, $a_{\max }=(-34749+4617 \sqrt{57}) / 64$. Together with the superconformal conditions, we can solve for the R-charges of the bifundamentals. Then the R-charges of GLSM fields should satisfy

$$
\begin{aligned}
\left(4 p_{2}+\right. & \left.4 p_{3}+2 p_{4}\right) p_{5}^{2}+\left(4 p_{2}^{2}+8 p_{3} p_{2}+8 p_{4} p_{2}-8 p_{2}+4 p_{3}^{2}+2 p_{4}^{2}-8 p_{3}+8 p_{3} p_{4}-4 p_{4}\right) p_{5} \\
= & -4 p_{3} p_{2}^{2}-12 p_{4} p_{2}^{2}-4 p_{3}^{2} p_{2}-12 p_{4}^{2} p_{2}+8 p_{3} p_{2}-24 p_{3} p_{4} p_{2}+24 p_{4} p_{2}-10 p_{3} p_{4}^{2} \\
& \quad-10 p_{3}^{2} p_{4}+20 p_{3} p_{4}-171 \sqrt{57}+1287
\end{aligned}
$$

constrained by $\sum_{i=1}^{5} p_{i}=2$ and $0<p_{i}<2$, with others vanishing.

\subsection{Polytope 35: $K^{4,4,2,4}$}

The polytope is

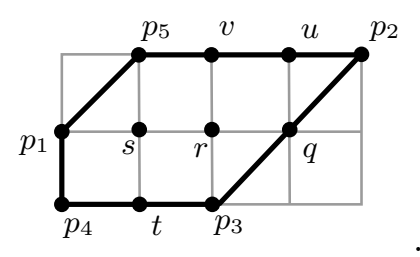


The brane tiling and the corrresponding quiver are
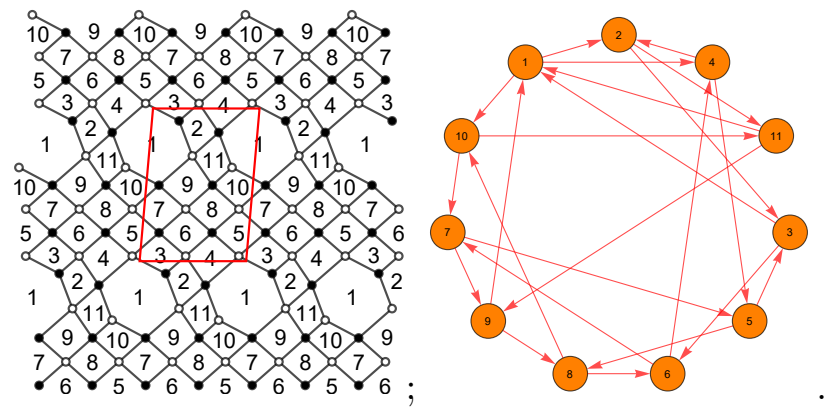

The superpotential is

$$
\begin{aligned}
W= & X_{1,4} X_{4,5} X_{5,3} X_{3,1}+X_{2,3} X_{3,6} X_{6,4} X_{4,2}+X_{5,8} X_{8,10} X_{10,7} X_{7,5}+X_{6,7} X_{7,9} X_{9,8} X_{8,6} \\
& +X_{10,11} X_{11,1} X_{1,10}+X_{9,1} X_{1,2} X_{2,11} X_{11,9}-X_{1,2} X_{2,3} X_{3,1}-X_{2,11} X_{11,1} X_{1,4} X_{4,2} \\
& -X_{5,3} X_{3,6} X_{6,7} X_{7,5}-X_{6,4} X_{4,5} X_{5,8} X_{8,6}-X_{9,8} X_{8,10} X_{10,11} X_{11,9}-X_{10,7} X_{7,9} X_{9,1} X_{1,10}
\end{aligned}
$$

The number of perfect matchings is $c=60$, which leads to gigantic $P, Q_{t}$ and $G_{t}$. Hence, we will not list them here. The GLSM fields associated to each point are shown in (5.87), where

$$
\begin{array}{lll}
q=\left\{q_{1}, q_{2}\right\}, & r=\left\{r_{1}, \ldots, r_{25}\right\}, & u=\left\{u_{1}, \ldots, u_{3}\right\}, \\
t=\left\{t_{1}, t_{2}\right\}, & s=\left\{s_{1}, \ldots, s_{20}\right\}, & v=\left\{v_{1}, \ldots, v_{3}\right\} .
\end{array}
$$

The mesonic symmetry reads $\mathrm{U}(1)^{2} \times \mathrm{U}(1)_{\mathrm{R}}$ and the baryonic symmetry reads $\mathrm{U}(1)_{\mathrm{h}}^{4} \times \mathrm{U}(1)^{6}$, where the subscripts "R" and "h" indicate R- and hidden symmetries respectively.

The Hilbert series of the toric cone is

$$
\begin{aligned}
H S= & \frac{1}{\left(1-\frac{t_{3}^{2}}{t_{1}}\right)\left(1-\frac{t_{3}}{t_{2}}\right)\left(1-\frac{t_{1} t_{2}}{t_{3}^{2}}\right)}+\frac{1}{\left(1-t_{2}\right)\left(1-\frac{t_{1}}{t_{3}}\right)\left(1-\frac{t_{3}^{2}}{t_{1} t_{2}}\right)} \\
& +\frac{1}{\left(1-\frac{1}{t_{2}}\right)\left(1-\frac{t_{1}}{t_{3}}\right)\left(1-\frac{t_{2} t_{3}^{2}}{t_{1}}\right)}+\frac{1}{\left(1-\frac{t_{1}}{t_{3}^{2}}\right)\left(1-\frac{t_{3}}{t_{2}}\right)\left(1-\frac{t_{2} t_{3}^{2}}{t_{1}}\right)} \\
& +\frac{1}{\left(1-t_{1}\right)\left(1-t_{2}\right)\left(1-\frac{t_{3}}{t_{1} t_{2}}\right)}+\frac{1}{\left(1-\frac{1}{t_{1}}\right)\left(1-t_{2}\right)\left(1-\frac{t_{1} t_{3}}{t_{2}}\right)} \\
& +\frac{1}{\left(1-t_{1}\right)\left(1-\frac{1}{t_{1} t_{2}}\right)\left(1-t_{2} t_{3}\right)}+\frac{1}{\left(1-t_{1} t_{3}\right)\left(1-t_{2} t_{3}\right)\left(1-\frac{1}{t_{1} t_{2} t_{3}}\right)} \\
& +\frac{1}{\left(1-\frac{1}{t_{1}}\right)\left(1-\frac{1}{t_{2}}\right)\left(1-t_{1} t_{2} t_{3}\right)}+\frac{1}{\left(1-\frac{1}{t_{2}}\right)\left(1-t_{1} t_{2}\right)\left(1-\frac{t_{3}}{t_{1}}\right)} \\
& +\frac{1}{\left(1-\frac{t_{3}}{t_{1}}\right)\left(1-\frac{t_{3}}{t_{2}}\right)\left(1-\frac{t_{1} t_{2}}{t_{3}}\right)} .
\end{aligned}
$$

The volume function is then

$$
V=-\frac{-b_{1}\left(b_{2}+15\right)+b_{2}^{2}+3 b_{2}-72}{\left(b_{1}+3\right)\left(b_{2}-3\right)\left(b_{2}+3\right)\left(b_{1}-b_{2}-6\right)\left(b_{1}-b_{2}+3\right)} .
$$


Minimizing $V$ yields $V_{\min }=0.112411$ at $b_{1}=2.224267, b_{2}=0.261487$. Thus, $a_{\max }=$ 2.223982. Together with the superconformal conditions, we can solve for the R-charges of the bifundamentals. Then the R-charges of GLSM fields should satisfy

$$
\begin{aligned}
& \left(12.1849 p_{2}+4.06163 p_{4}+6.09245 p_{5}\right) p_{3}^{2}+\left(12.1849 p_{2}^{2}+16.2465 p_{4} p_{2}+12.1849 p_{5} p_{2}\right. \\
& \left.-24.3698 p_{2}+4.06163 p_{4}^{2}+6.09245 p_{5}^{2}-8.12326 p_{4}+4.06163 p_{4} p_{5}-12.1849 p_{5}\right) p_{3} \\
& =-8.12326 p_{4} p_{2}^{2}-6.09245 p_{5} p_{2}^{2}-8.12326 p_{4}^{2} p_{2}-6.09245 p_{5}^{2} p_{2}+16.2465 p_{4} p_{2}-4.06163 p_{4} p_{5} p_{2} \\
& \quad+12.1849 p_{5} p_{2}-2.03082 p_{4} p_{5}^{2}-2.03082 p_{4}^{2} p_{5}+4.06163 p_{4} p_{5}-5.35289
\end{aligned}
$$

constrained by $\sum_{i=1}^{5} p_{i}=2$ and $0<p_{i}<2$, with others vanishing.

\subsection{Polytope 36: $K^{4,4,2,2}$}

The polytope is

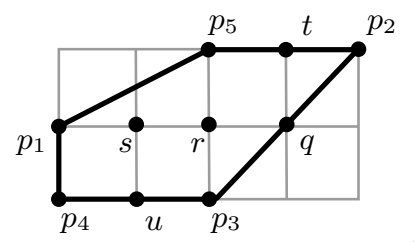

The brane tiling and the corrresponding quiver are
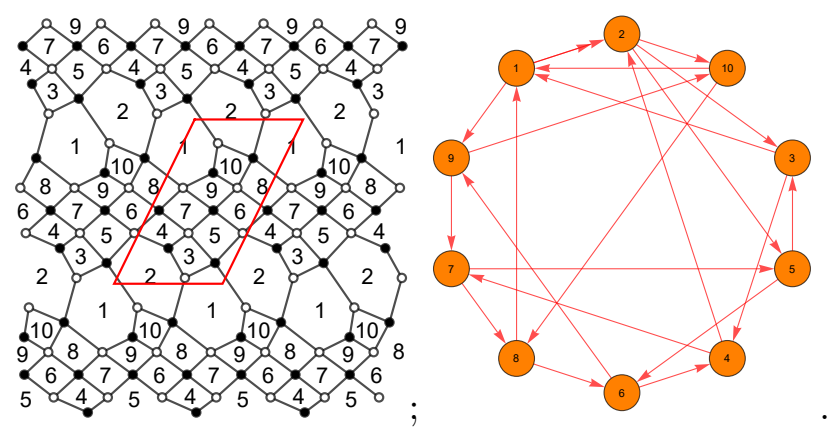

The superpotential is

$$
\begin{aligned}
W= & X_{12}^{1} X_{23} X_{31}+X_{25} X_{56} X_{64} X_{42}+X_{47} X_{75} X_{53} X_{34}+X_{69} X_{9,10} X_{10,8} X_{86} \\
& +X_{78} X_{81} X_{19} X_{97}+X_{10,1} X_{12}^{2} X_{2,10}-X_{12}^{2} X_{25} X_{53} X_{31}-X_{23} X_{34} X_{42} \\
& -X_{75} X_{56} X_{69} X_{97}-X_{64} X_{47} X_{78} X_{86}-X_{10,8} X_{81} X_{12}^{1} X_{2,10}-X_{19} X_{9,10} X_{10,1}
\end{aligned}
$$

The number of perfect matchings is $c=48$, which leads to gigantic $P, Q_{t}$ and $G_{t}$. Hence, we will not list them here. The GLSM fields associated to each point are shown in (5.94), where

$$
\begin{aligned}
q & =\left\{q_{1}, q_{2}\right\}, & r & =\left\{r_{1}, \ldots, r_{21}\right\}, \\
t & =\left\{t_{1}, t_{2}\right\}, & s & =\left\{s_{1}, \ldots, s_{16}\right\} .
\end{aligned} \quad u=\left\{u_{1}, u_{2}\right\},
$$

The mesonic symmetry reads $\mathrm{U}(1)^{2} \times \mathrm{U}(1)_{\mathrm{R}}$ and the baryonic symmetry reads $\mathrm{U}(1)_{\mathrm{h}}^{4} \times \mathrm{U}(1)^{5}$, where the subscripts "R" and "h" indicate R- and hidden symmetries respectively. 
The Hilbert series of the toric cone is

$$
\begin{aligned}
H S= & \frac{1}{\left(1-t_{2}\right)\left(1-\frac{t_{2}}{t_{1}}\right)\left(1-\frac{t_{1} t_{3}}{t_{2}^{2}}\right)}+\frac{1}{\left(1-t_{2}\right)\left(1-\frac{t_{3}^{2}}{t_{1}}\right)\left(1-\frac{t_{1}}{t_{2} t_{3}}\right)} \\
& +\frac{1}{\left(1-\frac{1}{t_{2}}\right)\left(1-\frac{t_{1}}{t_{3}}\right)\left(1-\frac{t_{2} t_{3}^{2}}{t_{1}}\right)}+\frac{1}{\left(1-\frac{t_{1}}{t_{3}^{2}}\right)\left(1-\frac{t_{3}}{t_{2}}\right)\left(1-\frac{t_{2} t_{3}^{2}}{t_{1}}\right)} \\
& +\frac{1}{\left(1-\frac{1}{t_{2}}\right)\left(1-\frac{t_{2}}{t_{1}}\right)\left(1-t_{1} t_{3}\right)}+\frac{1}{\left(1-\frac{1}{t_{1}}\right)\left(1-\frac{t_{1}}{t_{2}}\right)\left(1-t_{2} t_{3}\right)} \\
& +\frac{1}{\left(1-t_{1}\right)\left(1-\frac{1}{t_{2}}\right)\left(1-\frac{t_{2} t_{3}}{t_{1}}\right)}+\frac{1}{\left(1-\frac{t_{1}}{t_{3}}\right)\left(1-\frac{t_{3}}{t_{2}}\right)\left(1-\frac{t_{2} t_{3}}{t_{1}}\right)} \\
& +\frac{1}{\left(1-t_{2}\right)\left(1-\frac{t_{1}}{t_{2}}\right)\left(1-\frac{t_{3}}{t_{1}}\right)}+\frac{1}{\left(1-\frac{t_{3}}{t_{1}}\right)\left(1-t_{2} t_{3}\right)\left(1-\frac{t_{1}}{t_{2} t_{3}}\right)} .
\end{aligned}
$$

The volume function is then

$$
V=-\frac{2\left(-6 b_{1}+b_{2}^{2}+6 b_{2}-45\right)}{\left(b_{1}+3\right)\left(b_{2}-3\right)\left(b_{2}+3\right)\left(b_{1}-2 b_{2}+3\right)\left(b_{1}-b_{2}-6\right)} .
$$

Minimizing $V$ yields $V_{\min }=(59+11 \sqrt{33}) / 972$ at $b_{1}=(9 \sqrt{33}-33) / 8, b_{2}=0$. Thus, $a_{\max }=\frac{243}{512}(11 \sqrt{33}-59)$. Together with the superconformal conditions, we can solve for the R-charges of the bifundamentals. Then the R-charges of GLSM fields should satisfy

$$
\begin{aligned}
& \left(19683 p_{2}+6561 p_{4}+13122 p_{5}\right) p_{3}^{2}+\left(19683 p_{2}^{2}+26244 p_{4} p_{2}+26244 p_{5} p_{2}-39366 p_{2}\right. \\
& \left.+6561 p_{4}^{2}+13122 p_{5}^{2}-13122 p_{4}+13122 p_{4} p_{5}-26244 p_{5}\right) p_{3}=-13122 p_{4} p_{2}^{2}-6561 p_{5} p_{2}^{2} \\
& -13122 p_{4}^{2} p_{2}-6561 p_{5}^{2} p_{2}+26244 p_{4} p_{2}-13122 p_{4} p_{5} p_{2}+13122 p_{5} p_{2}-6561 p_{4} p_{5}^{2}-6561 p_{4}^{2} p_{5} \\
& +13122 p_{4} p_{5}-4 \sqrt{33}-236
\end{aligned}
$$

constrained by $\sum_{i=1}^{5} p_{i}=2$ and $0<p_{i}<2$, with others vanishing.

\subsection{Polytope 37: $K^{2,4,1,3}$}

The polytope is

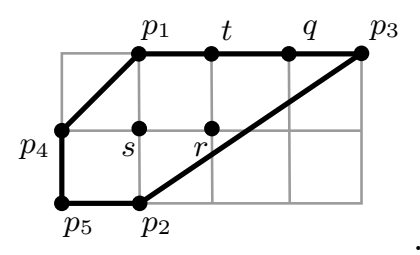


The brane tiling and the corrresponding quiver are
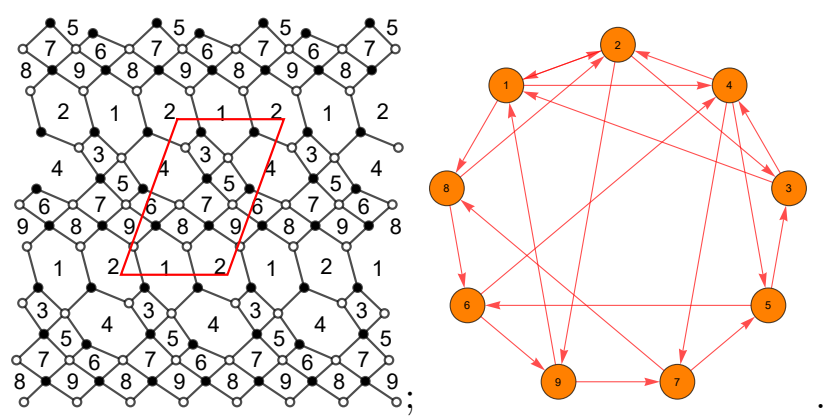

The superpotential is

$$
\begin{aligned}
W= & X_{23} X_{34} X_{42}+X_{14} X_{45} X_{53} X_{31}+X_{47} X_{78} X_{86} X_{64}+X_{56} X_{69} X_{97} X_{75} \\
& +X_{82} X_{21} X_{18}+X_{91} X_{12} X_{29}-X_{12} X_{23} X_{31}-X_{21} X_{14} X_{42} \\
& -X_{34} X_{47} X_{75} X_{53}-X_{45} X_{56} X_{64}-X_{97} X_{78} X_{82} X_{29}-X_{86} X_{69} X_{91} X_{18}
\end{aligned}
$$

The number of perfect matchings is $c=34$, which leads to gigantic $P, Q_{t}$ and $G_{t}$. Hence, we will not list them here. The GLSM fields associated to each point are shown in (5.101), where

$$
q=\left\{q_{1}, \ldots, q_{3}\right\}, \quad r=\left\{r_{1}, \ldots, r_{9}\right\}, \quad s=\left\{s_{1}, \ldots, s_{14}\right\}, \quad t=\left\{t_{1}, \ldots, t_{3}\right\} .
$$

The mesonic symmetry reads $\mathrm{U}(1)^{2} \times \mathrm{U}(1)_{\mathrm{R}}$ and the baryonic symmetry reads $\mathrm{U}(1)_{\mathrm{h}}^{4} \times \mathrm{U}(1)^{4}$, where the subscripts "R" and "h" indicate R- and hidden symmetries respectively.

The Hilbert series of the toric cone is

$$
\begin{aligned}
H S= & \frac{1}{\left(1-\frac{t_{1}}{t_{2}^{2} t_{3}}\right)\left(1-\frac{t_{1}}{t_{2} t_{3}}\right)\left(1-\frac{t_{2}^{3} t_{3}^{3}}{t_{1}^{2}}\right)}+\frac{1}{\left(1-\frac{t_{3}}{t_{2}}\right)\left(1-\frac{t_{1}}{t_{2} t_{3}}\right)\left(1-\frac{t_{2}^{2} t_{3}}{t_{1}}\right)} \\
& +\frac{1}{\left(1-t_{1}\right)\left(1-t_{2}\right)\left(1-\frac{t_{3}}{t_{1} t_{2}}\right)}+\frac{1}{\left(1-\frac{1}{t_{1}}\right)\left(1-t_{2}\right)\left(1-\frac{t_{1} t_{3}}{t_{2}}\right)} \\
& +\frac{1}{\left(1-t_{1} t_{3}\right)\left(1-t_{2} t_{3}\right)\left(1-\frac{1}{t_{1} t_{2} t_{3}}\right)}+\frac{1}{\left(1-t_{1}\right)\left(1-\frac{1}{t_{2}}\right)\left(1-\frac{t_{2} t_{3}}{t_{1}}\right)} \\
& +\frac{1}{\left(1-\frac{1}{t_{1}}\right)\left(1-\frac{1}{t_{2}}\right)\left(1-t_{1} t_{2} t_{3}\right)}+\frac{1}{\left(1-\frac{t_{3}}{t_{1}}\right)\left(1-\frac{t_{3}}{t_{2}}\right)\left(1-\frac{t_{1} t_{2}}{t_{3}}\right)} \\
& +\frac{1}{\left(1-\frac{t_{1}}{t_{3}}\right)\left(1-\frac{t_{3}}{t_{2}}\right)\left(1-\frac{t_{2} t_{3}}{t_{1}}\right)} .
\end{aligned}
$$

The volume function is then

$$
V=-\frac{3\left(b_{2}^{2}-2 b_{2}-39\right)-4 b_{1}\left(b_{2}+6\right)}{\left(b_{1}+3\right)\left(b_{2}-3\right)\left(b_{2}+3\right)\left(b_{1}-b_{2}+3\right)\left(2 b_{1}-3\left(b_{2}+3\right)\right)} .
$$

Minimizing $V$ yields $V_{\min }=0.133134$ at $b_{1}=1.844031, b_{2}=0.575732$. Thus, $a_{\max }=$ 1.877807. Together with the superconformal conditions, we can solve for the R-charges of 
the bifundamentals. Then the R-charges of GLSM fields should satisfy

$$
\begin{aligned}
& \left(9.05965 p_{3}+3.01988 p_{4}+3.01988 p_{5}\right) p_{2}^{2}+\left(9.05965 p_{3}^{2}+9.05965 p_{4} p_{3}+18.1193 p_{5} p_{3}\right. \\
& \left.-8.1193 p_{3}+3.01988 p_{4}^{2}+3.01988 p_{5}^{2}-6.03977 p_{4}+6.03977 p_{4} p_{5}-6.03977 p_{5}\right) p_{2} \\
& =-4.52983 p_{4} p_{3}^{2}-9.05965 p_{5} p_{3}^{2}-4.52983 p_{4}^{2} p_{3}-9.05965 p_{5}^{2} p_{3}+9.05965 p_{4} p_{3}-9.05965 p_{4} p_{5} p_{3} \\
& \quad+18.1193 p_{5} p_{3}-1.50994 p_{4} p_{5}^{2}-1.50994 p_{4}^{2} p_{5}+3.01988 p_{4} p_{5}-3.36045
\end{aligned}
$$

constrained by $\sum_{i=1}^{5} p_{i}=2$ and $0<p_{i}<2$, with others vanishing.

\subsection{Polytope 38: $K^{2,4,1,2}$}

The polytope is

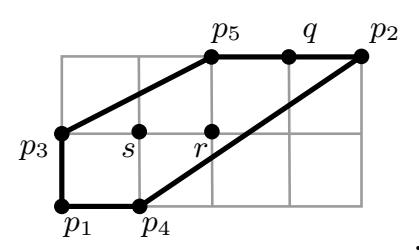

The brane tiling and the corrresponding quiver are
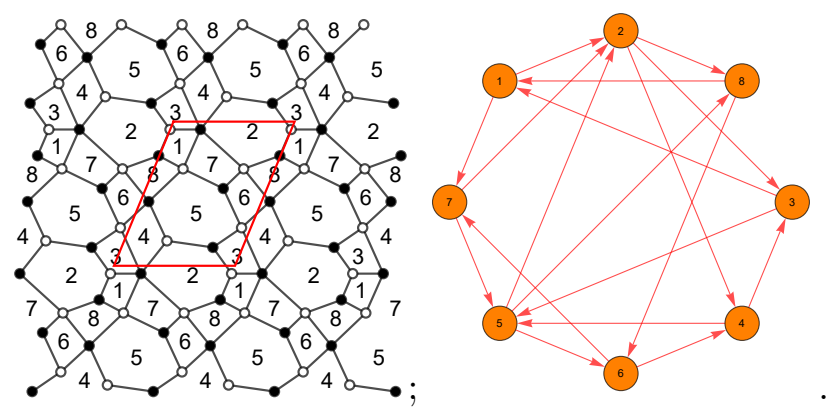

The superpotential is

$$
\begin{aligned}
W= & X_{12} X_{23} X_{31}+X_{24} X_{45} X_{52}+X_{35} X_{56} X_{64} X_{43}+X_{58} X_{81} X_{17} X_{75} \\
& +X_{67} X_{72} X_{28} X_{86}-X_{17} X_{72} X_{24} X_{43} X_{31}-X_{23} X_{35} X_{52}-X_{45} X_{58} X_{86} X_{64} \\
& -X_{56} X_{67} X_{75}-X_{28} X_{81} X_{12} .
\end{aligned}
$$


The perfect matching matrix is

$$
P=\left(\begin{array}{l|llllllllllllllllllllllll} 
& p_{1} & r_{1} & s_{1} & r_{2} & p_{2} & q_{1} & s_{2} & r_{3} & s_{3} & s_{4} & s_{5} & p_{3} & r_{4} & s_{6} & r_{5} & r_{6} & r_{7} & s_{7} & p_{4} & s_{8} & q_{2} & p_{5} & r_{8} & s_{9} \\
\hline X_{12} & 0 & 0 & 0 & 1 & 1 & 1 & 1 & 1 & 0 & 0 & 0 & 0 & 1 & 1 & 0 & 0 & 0 & 0 & 0 & 1 & 1 & 1 & 0 & 0 \\
X_{17} & 1 & 0 & 0 & 1 & 0 & 0 & 1 & 0 & 0 & 0 & 0 & 0 & 0 & 0 & 0 & 0 & 0 & 0 & 0 & 1 & 0 & 0 & 0 & 0 \\
X_{23} & 1 & 1 & 0 & 0 & 0 & 0 & 0 & 0 & 0 & 1 & 1 & 0 & 0 & 0 & 0 & 1 & 1 & 0 & 1 & 0 & 0 & 0 & 1 & 0 \\
X_{24} & 0 & 1 & 0 & 0 & 1 & 0 & 0 & 0 & 0 & 0 & 1 & 0 & 0 & 0 & 0 & 0 & 1 & 0 & 0 & 0 & 1 & 0 & 1 & 0 \\
X_{28} & 1 & 1 & 1 & 0 & 0 & 0 & 0 & 0 & 1 & 1 & 1 & 1 & 0 & 0 & 0 & 0 & 0 & 0 & 0 & 0 & 0 & 0 & 0 & 0 \\
X_{31} & 0 & 0 & 1 & 0 & 0 & 0 & 0 & 0 & 1 & 0 & 0 & 1 & 0 & 0 & 1 & 0 & 0 & 1 & 0 & 0 & 0 & 0 & 0 & 1 \\
X_{35} & 0 & 0 & 0 & 1 & 1 & 0 & 0 & 0 & 1 & 0 & 0 & 0 & 1 & 0 & 1 & 0 & 0 & 0 & 0 & 0 & 1 & 0 & 0 & 0 \\
X_{43} & 0 & 0 & 0 & 0 & 0 & 1 & 0 & 0 & 0 & 1 & 0 & 0 & 0 & 0 & 0 & 1 & 0 & 0 & 0 & 0 & 0 & 1 & 0 & 0 \\
X_{45} & 1 & 0 & 0 & 1 & 0 & 0 & 0 & 0 & 1 & 1 & 0 & 0 & 1 & 0 & 1 & 1 & 0 & 0 & 1 & 0 & 0 & 0 & 0 & 0 \\
X_{52} & 0 & 0 & 1 & 0 & 0 & 1 & 1 & 1 & 0 & 0 & 0 & 1 & 0 & 1 & 0 & 0 & 0 & 1 & 0 & 1 & 0 & 1 & 0 & 1 \\
X_{56} & 1 & 1 & 1 & 0 & 0 & 0 & 0 & 1 & 0 & 0 & 0 & 0 & 0 & 0 & 0 & 0 & 0 & 0 & 1 & 1 & 0 & 0 & 1 & 1 \\
X_{58} & 0 & 1 & 1 & 0 & 1 & 1 & 0 & 1 & 0 & 0 & 0 & 0 & 0 & 0 & 0 & 0 & 0 & 0 & 0 & 0 & 0 & 0 & 0 & 0 \\
X_{64} & 0 & 0 & 0 & 0 & 0 & 0 & 1 & 0 & 0 & 0 & 1 & 1 & 0 & 1 & 0 & 0 & 1 & 1 & 0 & 0 & 0 & 0 & 0 & 0 \\
X_{67} & 0 & 0 & 0 & 1 & 1 & 1 & 1 & 0 & 0 & 0 & 0 & 0 & 0 & 0 & 1 & 1 & 1 & 1 & 0 & 0 & 0 & 0 & 0 & 0 \\
X_{72} & 0 & 0 & 0 & 0 & 0 & 0 & 0 & 1 & 0 & 0 & 0 & 0 & 1 & 1 & 0 & 0 & 0 & 0 & 1 & 0 & 0 & 0 & 0 & 0 \\
X_{75} & 0 & 0 & 0 & 0 & 0 & 0 & 0 & 0 & 1 & 1 & 1 & 1 & 1 & 1 & 0 & 0 & 0 & 0 & 0 & 0 & 1 & 1 & 0 & 0 \\
X_{81} & 0 & 0 & 0 & 0 & 0 & 0 & 0 & 0 & 0 & 0 & 0 & 0 & 0 & 0 & 1 & 1 & 1 & 1 & 1 & 0 & 0 & 0 & 1 & 1 \\
X_{86} & 0 & 0 & 0 & 0 & 0 & 0 & 0 & 0 & 0 & 0 & 0 & 0 & 0 & 0 & 0 & 0 & 0 & 0 & 0 & 1 & 1 & 1 & 1 & 1
\end{array}\right),
$$

where the relations between bifundamentals and GLSM fields can be directly read off. Then we can get the total charge matrix:

$$
Q_{t}=\left(\begin{array}{cccccccccccccccccccccccc}
p_{1} & r_{1} & s_{1} & r_{2} & p_{2} & q_{1} & s_{2} & r_{3} & s_{3} & s_{4} & s_{5} & p_{3} & r_{4} & s_{6} & r_{5} & r_{6} & r_{7} & s_{7} & p_{4} & s_{8} & q_{2} & p_{5} & r_{8} & s_{9} \\
\hline 0 & 0 & 0 & 1 & 0 & 0 & 0 & 0 & 0 & 0 & 0 & 0 & 0 & 0 & -1 & 0 & 0 & 0 & 0 & -1 & 0 & 0 & 0 & 1 \\
0 & -1 & 1 & 1 & 0 & 0 & 0 & 0 & 0 & 0 & 0 & 0 & 0 & 0 & -1 & 0 & 0 & 0 & 0 & -1 & 0 & 0 & 1 & 0 \\
0 & 0 & 1 & 1 & 0 & -1 & 0 & 0 & -1 & 0 & 0 & 0 & 0 & 0 & 0 & 0 & 0 & 0 & 0 & -1 & 0 & 1 & 0 & 0 \\
1 & -1 & 1 & 0 & 0 & 0 & 0 & 0 & -1 & 0 & 0 & 0 & 0 & 0 & 0 & 0 & 0 & 0 & 0 & -1 & 1 & 0 & 0 & 0 \\
-1 & 0 & 1 & 1 & 0 & 0 & 0 & -1 & 0 & 0 & 0 & 0 & 0 & 0 & -1 & 0 & 0 & 0 & 1 & 0 & 0 & 0 & 0 & 0 \\
0 & 0 & 0 & 1 & 0 & 0 & -1 & 0 & 0 & 0 & 0 & 0 & 0 & 0 & -1 & 0 & 0 & 1 & 0 & 0 & 0 & 0 & 0 & 0 \\
0 & -1 & 1 & 1 & 0 & 0 & -1 & 0 & 0 & 0 & 0 & 0 & 0 & 0 & -1 & 0 & 1 & 0 & 0 & 0 & 0 & 0 & 0 & 0 \\
-1 & 0 & 1 & 1 & 0 & -1 & 0 & 0 & 0 & 0 & 0 & 0 & 0 & 0 & -1 & 1 & 0 & 0 & 0 & 0 & 0 & 0 & 0 & 0 \\
0 & 0 & 1 & 1 & 0 & 0 & -1 & -1 & -1 & 0 & 0 & 0 & 0 & 1 & 0 & 0 & 0 & 0 & 0 & 0 & 0 & 0 & 0 & 0 \\
0 & 0 & 1 & 0 & 0 & 0 & 0 & -1 & -1 & 0 & 0 & 0 & 1 & 0 & 0 & 0 & 0 & 0 & 0 & 0 & 0 & 0 & 0 & 0 \\
0 & 0 & 0 & 1 & 0 & 0 & -1 & 0 & -1 & 0 & 0 & 1 & 0 & 0 & 0 & 0 & 0 & 0 & 0 & 0 & 0 & 0 & 0 & 0 \\
0 & -1 & 1 & 1 & 0 & 0 & -1 & 0 & -1 & 0 & 1 & 0 & 0 & 0 & 0 & 0 & 0 & 0 & 0 & 0 & 0 & 0 & 0 & 0 \\
-1 & 0 & 1 & 1 & 0 & -1 & 0 & 0 & -1 & 1 & 0 & 0 & 0 & 0 & 0 & 0 & 0 & 0 & 0 & 0 & 0 & 0 & 0 & 0 \\
1 & -1 & 0 & -1 & 1 & 0 & 0 & 0 & 0 & 0 & 0 & 0 & 0 & 0 & 0 & 0 & 0 & 0 & 0 & 0 & 0 & 0 & 0 & 0 \\
\hline 0 & 0 & 1 & 2 & 0 & 0 & 0 & 0 & 0 & 0 & 0 & 0 & 0 & 0 & -2 & 0 & 0 & 0 & 0 & -1 & 0 & 0 & 0 & 0 \\
0 & 0 & 1 & 2 & 0 & 0 & 0 & -1 & -1 & 0 & 0 & 0 & 0 & 0 & -1 & 0 & 0 & 0 & 0 & 0 & 0 & 0 & 0 & 0 \\
0 & 0 & 0 & 1 & 0 & 0 & -1 & 0 & 0 & 0 & 0 & 0 & 0 & 0 & -1 & 0 & 0 & 0 & 0 & 1 & 0 & 0 & 0 & 0 \\
0 & 0 & -1 & 1 & 0 & 0 & 0 & 0 & 1 & 0 & 0 & 0 & 0 & 0 & -1 & 0 & 0 & 0 & 0 & 0 & 0 & 0 & 0 & 0 \\
-1 & 1 & 0 & 1 & 0 & -1 & 1 & 0 & 0 & 0 & 0 & 0 & 0 & 0 & -1 & 0 & 0 & 0 & 0 & 0 & 0 & 0 & 0 & 0 \\
1 & 0 & -1 & 0 & 0 & 1 & 0 & 0 & 0 & 0 & 0 & 0 & 0 & 0 & -1 & 0 & 0 & 0 & 0 & 0 & 0 & 0 & 0 & 0 \\
0 & -1 & 0 & 1 & 0 & 0 & 0 & 1 & 0 & 0 & 0 & 0 & 0 & 0 & -1 & 0 & 0 & 0 & 0 & 0 & 0 & 0 & 0 & 0
\end{array}\right)
$$

with kernel

$$
G_{t}=\left(\begin{array}{cccccccccccccccccccccccc}
p_{1} & r_{1} & s_{1} & r_{2} & p_{2} & q_{1} & s_{2} & r_{3} & s_{3} & s_{4} & s_{5} & p_{3} & r_{4} & s_{6} & r_{5} & r_{6} & r_{7} & s_{7} & p_{4} & s_{8} & q_{2} & p_{5} & r_{8} & s_{9} \\
\hline 2 & 0 & 1 & 0 & -2 & -1 & 1 & 0 & 1 & 1 & 1 & 2 & 0 & 1 & 0 & 0 & 0 & 1 & 1 & 1 & -1 & 0 & 0 & 1 \\
0 & 1 & 0 & 1 & 2 & 1 & 0 & 1 & 0 & 0 & 0 & -1 & 1 & 0 & 1 & 1 & 1 & 0 & 1 & 0 & 1 & 0 & 1 & 0 \\
1 & 1 & 1 & 1 & 1 & 1 & 1 & 1 & 1 & 1 & 1 & 1 & 1 & 1 & 1 & 1 & 1 & 1 & 1 & 1 & 1 & 1 & 1 & 1
\end{array}\right)
$$

From $G_{t}$, we can get the GLSM fields associated to each point as shown in (5.108), where

$$
q=\left\{q_{1}, q_{2}\right\}, r=\left\{r_{1}, \ldots, r_{8}\right\}, s=\left\{s_{1}, \ldots, s_{9}\right\} .
$$

From $Q_{t}$ (and $Q_{F}$ ), the mesonic symmetry reads $\mathrm{U}(1)^{2} \times \mathrm{U}(1)_{\mathrm{R}}$ and the baryonic symmetry reads $\mathrm{U}(1)_{\mathrm{h}}^{4} \times \mathrm{U}(1)^{3}$, where the subscripts " $\mathrm{R}$ " and " $\mathrm{h}$ " indicate $\mathrm{R}$ - and hidden symmetries respectively. 
The Hilbert series of the toric cone is

$$
\begin{aligned}
H S= & \frac{1}{\left(1-\frac{t_{1}}{t_{2}^{2} t_{3}}\right)\left(1-\frac{t_{1}}{t_{2} t_{3}}\right)\left(1-\frac{t_{2}^{3} t_{3}^{3}}{t_{1}^{2}}\right)}+\frac{1}{\left(1-t_{2}\right)\left(1-\frac{t_{2}}{t_{1}}\right)\left(1-\frac{t_{1} t_{3}}{t_{2}^{2}}\right)} \\
& +\frac{1}{\left(1-\frac{t_{3}}{t_{2}}\right)\left(1-\frac{t_{1}}{t_{2} t_{3}}\right)\left(1-\frac{t_{2}^{2} t_{3}}{t_{1}}\right)}+\frac{1}{\left(1-t_{1} t_{3}\right)\left(1-t_{2} t_{3}\right)\left(1-\frac{1}{t_{1} t_{2} t_{3}}\right)} \\
& +\frac{1}{\left(1-t_{1}\right)\left(1-\frac{1}{t_{2}}\right)\left(1-\frac{t_{2} t_{3}}{t_{1}}\right)}+\frac{1}{\left(1-\frac{t_{1}}{t_{3}}\right)\left(1-\frac{t_{3}}{t_{2}}\right)\left(1-\frac{t_{2} t_{3}}{t_{1}}\right)} \\
& +\frac{1}{\left(1-\frac{1}{t_{1}}\right)\left(1-\frac{1}{t_{2}}\right)\left(1-t_{1} t_{2} t_{3}\right)}+\frac{1}{\left(1-t_{2}\right)\left(1-\frac{t_{1}}{t_{2}}\right)\left(1-\frac{t_{3}}{t_{1}}\right)} .
\end{aligned}
$$

The volume function is then

$$
V=\frac{6\left(b_{2}^{2}+b_{2}-24\right)-2 b_{1}\left(b_{2}+9\right)}{\left(b_{1}+3\right)\left(b_{2}-3\right)\left(b_{2}+3\right)\left(b_{1}-2 b_{2}+3\right)\left(2 b_{1}-3\left(b_{2}+3\right)\right)} .
$$

Minimizing $V$ yields $V_{\min }=0.154554$ at $b_{1}=1.904961, b_{2}=0.289299$. Thus, $a_{\max }=$ 1.617558. Together with the superconformal conditions, we can solve for the R-charges of the bifundamentals. Then the R-charges of GLSM fields should satisfy

$$
\begin{aligned}
& \left(0.5625 p_{3}+0.28125 p_{4}+0.5625 p_{5}\right) p_{2}^{2}+\left(0.5625 p_{3}^{2}+0.28125 p_{4} p_{3}+1.125 p_{5} p_{3}\right. \\
& \left.-1.125 p_{3}+0.28125 p_{4}^{2}+0.5625 p_{5}^{2}-0.5625 p_{4}+0.5625 p_{4} p_{5}-1.125 p_{5}\right) p_{2}=-0.140625 p_{4} p_{3}^{2} \\
& -0.28125 p_{5} p_{3}^{2}-0.140625 p_{4}^{2} p_{3}-0.28125 p_{5}^{2} p_{3}+0.28125 p_{4} p_{3}-0.28125 p_{4} p_{5} p_{3}+0.5625 p_{5} p_{3} \\
& -0.28125 p_{4} p_{5}^{2}-0.28125 p_{4}^{2} p_{5}+0.5625 p_{4} p_{5}-0.269593
\end{aligned}
$$

constrained by $\sum_{i=1}^{5} p_{i}=2$ and $0<p_{i}<2$, with others vanishing.

\subsection{Polytope 39: $K^{2,4,1,1}$}

The polytope is

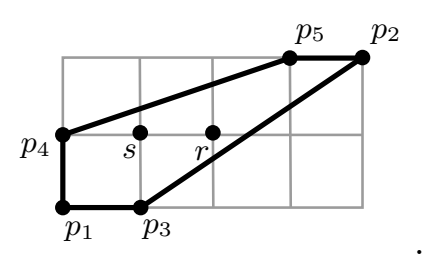

The brane tiling and the corrresponding quiver are
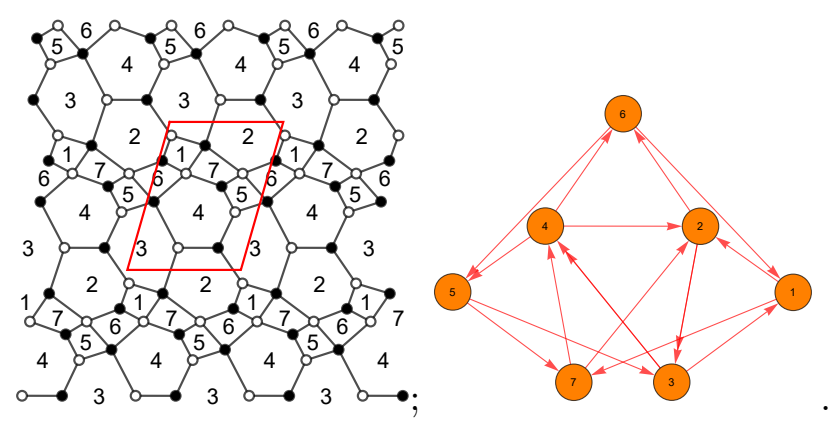
The superpotential is

$$
\begin{aligned}
W= & X_{12} X_{23}^{1} X_{31}+X_{23}^{2} X_{34}^{1} X_{42}+X_{34}^{2} X_{45} X_{53}+X_{57} X_{72} X_{26} X_{65} \\
& +X_{46} X_{61} X_{17} X_{74}-X_{17} X_{72} X_{23}^{2} X_{31}-X_{23}^{1} X_{34}^{2} X_{42}-X_{34}^{1} X_{46} X_{65} X_{53} \\
& -X_{45} X_{57} X_{74}-X_{26} X_{61} X_{12} .
\end{aligned}
$$

The perfect matching matrix is

$$
P=\left(\begin{array}{c|cccccccccccccccccccc} 
& p_{1} & r_{1} & s_{1} & r_{2} & p_{2} & s_{2} & r_{3} & r_{4} & s_{3} & s_{4} & r_{5} & s_{5} & r_{6} & p_{3} & s_{6} & s_{7} & p_{4} & p_{5} & r_{7} & s_{8} \\
\hline X_{12} & 0 & 0 & 0 & 1 & 1 & 1 & 0 & 0 & 0 & 1 & 0 & 0 & 1 & 0 & 0 & 0 & 0 & 1 & 1 & 1 \\
X_{17} & 1 & 0 & 0 & 1 & 0 & 1 & 0 & 0 & 0 & 1 & 0 & 0 & 0 & 0 & 0 & 0 & 0 & 0 & 0 & 0 \\
X_{23}^{1} & 1 & 1 & 0 & 0 & 0 & 0 & 0 & 1 & 0 & 0 & 1 & 0 & 0 & 1 & 0 & 1 & 0 & 0 & 0 & 0 \\
X_{23}^{2} & 0 & 1 & 0 & 0 & 1 & 0 & 0 & 1 & 0 & 0 & 1 & 0 & 0 & 0 & 0 & 1 & 0 & 1 & 0 & 0 \\
X_{26} & 1 & 1 & 1 & 0 & 0 & 0 & 0 & 0 & 0 & 0 & 0 & 0 & 0 & 0 & 1 & 1 & 1 & 0 & 0 & 0 \\
X_{31} & 0 & 0 & 1 & 0 & 0 & 0 & 1 & 0 & 1 & 0 & 0 & 1 & 0 & 0 & 1 & 0 & 1 & 0 & 0 & 0 \\
X_{34}^{1} & 1 & 0 & 0 & 1 & 0 & 0 & 1 & 0 & 0 & 0 & 0 & 0 & 0 & 1 & 1 & 0 & 0 & 0 & 1 & 0 \\
X_{34}^{2} & 0 & 0 & 0 & 1 & 1 & 0 & 1 & 0 & 0 & 0 & 0 & 0 & 0 & 0 & 1 & 0 & 0 & 1 & 1 & 0 \\
X_{42} & 0 & 0 & 1 & 0 & 0 & 1 & 0 & 0 & 1 & 1 & 0 & 1 & 1 & 0 & 0 & 0 & 1 & 0 & 0 & 1 \\
X_{45} & 1 & 1 & 1 & 0 & 0 & 0 & 0 & 0 & 0 & 1 & 1 & 1 & 1 & 1 & 0 & 0 & 0 & 0 & 0 & 0 \\
X_{46} & 0 & 1 & 1 & 0 & 1 & 0 & 0 & 0 & 0 & 0 & 0 & 0 & 1 & 0 & 0 & 0 & 0 & 0 & 0 & 0 \\
X_{53} & 0 & 0 & 0 & 0 & 0 & 1 & 0 & 1 & 1 & 0 & 0 & 0 & 0 & 0 & 0 & 1 & 1 & 0 & 0 & 1 \\
X_{57} & 0 & 0 & 0 & 1 & 1 & 1 & 1 & 1 & 1 & 0 & 0 & 0 & 0 & 0 & 0 & 0 & 0 & 0 & 0 & 0 \\
X_{61} & 0 & 0 & 0 & 0 & 0 & 0 & 1 & 1 & 1 & 0 & 1 & 1 & 0 & 1 & 0 & 0 & 0 & 0 & 0 & 0 \\
X_{65} & 0 & 0 & 0 & 0 & 0 & 0 & 0 & 0 & 0 & 1 & 1 & 1 & 0 & 0 & 0 & 0 & 0 & 1 & 0 & 0 \\
X_{72} & 0 & 0 & 0 & 0 & 0 & 0 & 0 & 0 & 0 & 0 & 0 & 0 & 1 & 1 & 0 & 0 & 0 & 0 & 1 & 1 \\
X_{74} & 0 & 0 & 0 & 0 & 0 & 0 & 0 & 0 & 0 & 0 & 0 & 0 & 0 & 0 & 1 & 1 & 1 & 1 & 1 & 1
\end{array}\right),
$$

where the relations between bifundamentals and GLSM fields can be directly read off. Then we can get the total charge matrix:

$$
Q_{t}=\left(\begin{array}{cccccccccccccccccccc}
p_{1} & r_{1} & s_{1} & r_{2} & p_{2} & s_{2} & r_{3} & r_{4} & s_{3} & s_{4} & r_{5} & s_{5} & r_{6} & p_{3} & s_{6} & s_{7} & p_{4} & p_{5} & r_{7} & s_{8} \\
\hline 0 & 0 & 1 & 1 & 0 & -1 & 0 & 0 & 0 & 0 & 0 & 0 & -1 & 0 & -1 & 0 & 0 & 0 & 0 & 1 \\
0 & 0 & 1 & 0 & 0 & 0 & 0 & 0 & 0 & 0 & 0 & 0 & -1 & 0 & -1 & 0 & 0 & 0 & 1 & 0 \\
1 & -1 & 1 & 0 & 0 & 0 & 0 & 0 & 0 & -1 & 0 & 0 & 0 & 0 & -1 & 0 & 0 & 1 & 0 & 0 \\
0 & 0 & 0 & 1 & 0 & -1 & 0 & 0 & 0 & 0 & 0 & 0 & 0 & 0 & -1 & 0 & 1 & 0 & 0 & 0 \\
0 & -1 & 1 & 1 & 0 & -1 & 0 & 0 & 0 & 0 & 0 & 0 & 0 & 0 & -1 & 1 & 0 & 0 & 0 & 0 \\
-1 & 0 & 1 & 1 & 0 & 0 & -1 & 0 & 0 & 0 & 0 & 0 & -1 & 1 & 0 & 0 & 0 & 0 & 0 & 0 \\
0 & 0 & 0 & 1 & 0 & 0 & -1 & 0 & 0 & -1 & 0 & 1 & 0 & 0 & 0 & 0 & 0 & 0 & 0 & 0 \\
0 & -1 & 1 & 1 & 0 & 0 & -1 & 0 & 0 & -1 & 1 & 0 & 0 & 0 & 0 & 0 & 0 & 0 & 0 & 0 \\
0 & 0 & 0 & 1 & 0 & -1 & -1 & 0 & 1 & 0 & 0 & 0 & 0 & 0 & 0 & 0 & 0 & 0 & 0 & 0 \\
0 & -1 & 1 & 1 & 0 & -1 & -1 & 1 & 0 & 0 & 0 & 0 & 0 & 0 & 0 & 0 & 0 & 0 & 0 & 0 \\
1 & -1 & 0 & -1 & 1 & 0 & 0 & 0 & 0 & 0 & 0 & 0 & 0 & 0 & 0 & 0 & 0 & 0 & 0 & 0 \\
\hline 0 & 0 & 1 & 2 & 0 & 0 & -1 & 0 & 0 & 0 & 0 & 0 & -1 & 0 & -1 & 0 & 0 & 0 & 0 & 0 \\
0 & 0 & 1 & 2 & 0 & 0 & -2 & 0 & 0 & -1 & 0 & 0 & 0 & 0 & 0 & 0 & 0 & 0 & 0 & 0 \\
0 & 0 & 0 & 1 & 0 & -1 & -1 & 0 & 0 & 1 & 0 & 0 & 0 & 0 & 0 & 0 & 0 & 0 & 0 & 0 \\
0 & 0 & -1 & 1 & 0 & 0 & -1 & 0 & 0 & 0 & 0 & 0 & 0 & 0 & 1 & 0 & 0 & 0 & 0 & 0 \\
0 & 1 & -1 & 0 & 0 & 1 & -1 & 0 & 0 & 0 & 0 & 0 & 0 & 0 & 0 & 0 & 0 & 0 & 0 & 0 \\
0 & -1 & 0 & 1 & 0 & 0 & -1 & 0 & 0 & 0 & 0 & 0 & 1 & 0 & 0 & 0 & 0 & 0 & 0 & 0
\end{array}\right)
$$

with kernel

$$
G_{t}=\left(\begin{array}{ccccccccccccccccccccc}
p_{1} & r_{1} & s_{1} & r_{2} & p_{2} & s_{2} & r_{3} & r_{4} & s_{3} & s_{4} & r_{5} & s_{5} & r_{6} & p_{3} & s_{6} & s_{7} & p_{4} & p_{5} & r_{7} & s_{8} \\
\hline 1 & 0 & 1 & 0 & -1 & 1 & 0 & 0 & 1 & 1 & 0 & 1 & 0 & 0 & 1 & 1 & 2 & 0 & 0 & 1 \\
1 & 1 & 0 & 1 & 1 & 0 & 1 & 1 & 0 & 0 & 1 & 0 & 1 & 2 & 0 & 0 & -1 & 0 & 1 & 0 \\
1 & 1 & 1 & 1 & 1 & 1 & 1 & 1 & 1 & 1 & 1 & 1 & 1 & 1 & 1 & 1 & 1 & 1 & 1 & 1
\end{array}\right) .
$$

From $G_{t}$, we can get the GLSM fields associated to each point as shown in (5.118), where

$$
r=\left\{r_{1}, \ldots, r_{7}\right\}, \quad s=\left\{s_{1}, \ldots, s_{8}\right\} .
$$

From $Q_{t}$ (and $Q_{F}$ ), the mesonic symmetry reads $\mathrm{U}(1)^{2} \times \mathrm{U}(1)_{\mathrm{R}}$ and the baryonic symmetry reads $\mathrm{U}(1)_{\mathrm{h}}^{4} \times \mathrm{U}(1)^{2}$, where the subscripts " $\mathrm{R}$ " and " $\mathrm{h}$ " indicate $\mathrm{R}$ - and hidden symmetries respectively. 
The Hilbert series of the toric cone is

$$
\begin{aligned}
H S= & \frac{1}{\left(1-\frac{t_{1}}{t_{2}^{2} t_{3}}\right)\left(1-\frac{t_{1}}{t_{2} t_{3}}\right)\left(1-\frac{t_{2}^{3} t_{3}^{3}}{t_{1}^{2}}\right)}+\frac{1}{\left(1-t_{2}\right)\left(1-\frac{t_{1}}{t_{2}^{2}}\right)\left(1-\frac{t_{2} t_{3}}{t_{1}}\right)} \\
& +\frac{1}{\left(1-\frac{t_{3}}{t_{2}}\right)\left(1-\frac{t_{1}}{t_{2} t_{3}}\right)\left(1-\frac{t_{2}^{2} t_{3}}{t_{1}}\right)}+\frac{1}{\left(1-t_{2}\right)\left(1-\frac{t_{2}^{2}}{t_{1}}\right)\left(1-\frac{t_{1} t_{3}}{t_{2}^{3}}\right)} \\
& +\frac{1}{\left(1-\frac{1}{t_{1}}\right)\left(1-\frac{t_{1}}{t_{2}}\right)\left(1-t_{2} t_{3}\right)}+\frac{1}{\left(1-t_{1}\right)\left(1-\frac{1}{t_{2}}\right)\left(1-\frac{t_{2} t_{3}}{t_{1}}\right)} \\
& +\frac{1}{\left(1-\frac{1}{t_{2}}\right)\left(1-\frac{t_{2}}{t_{1}}\right)\left(1-t_{1} t_{3}\right)} .
\end{aligned}
$$

The volume function is then

$$
V=\frac{3\left(4 b_{1}-3 b_{2}^{2}-6 b_{2}+57\right)}{\left(b_{1}+3\right)\left(b_{2}-3\right)\left(b_{2}+3\right)\left(b_{1}-3 b_{2}+3\right)\left(2 b_{1}-3\left(b_{2}+3\right)\right)} .
$$

Minimizing $V$ yields $V_{\min }=(347+29 \sqrt{145}) / 4050$ at $b_{1}=(15 \sqrt{145}-153) / 16, b_{2}=0$. Thus, $a_{\max }=\frac{675}{1024}(29 \sqrt{145}-347)$. Together with the superconformal conditions, we can solve for the R-charges of the bifundamentals. Then the R-charges of GLSM fields should satisfy

$$
\begin{aligned}
& \left(64 p_{3}+128 p_{4}+64 p_{5}\right) p_{2}^{2}+\left(64 p_{3}^{2}+64 p_{4} p_{3}+128 p_{5} p_{3}-128 p_{3}+128 p_{4}^{2}+64 p_{5}^{2}\right. \\
& \left.-256 p_{4}+256 p_{4} p_{5}-128 p_{5}\right) p_{2}=-32 p_{4} p_{3}^{2}-64 p_{5} p_{3}^{2}-32 p_{4}^{2} p_{3}-64 p_{5}^{2} p_{3}+64 p_{4} p_{3} \\
& -64 p_{4} p_{5} p_{3}+128 p_{5} p_{3}-96 p_{4} p_{5}^{2}-96 p_{4}^{2} p_{5}+192 p_{4} p_{5}-725 \sqrt{145}+8675
\end{aligned}
$$

constrained by $\sum_{i=1}^{5} p_{i}=2$ and $0<p_{i}<2$, with others vanishing.

\subsection{Polytope 40: $K^{4,3,2,2}$}

The polytope is

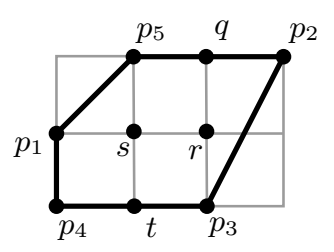

The brane tiling and the corrresponding quiver are
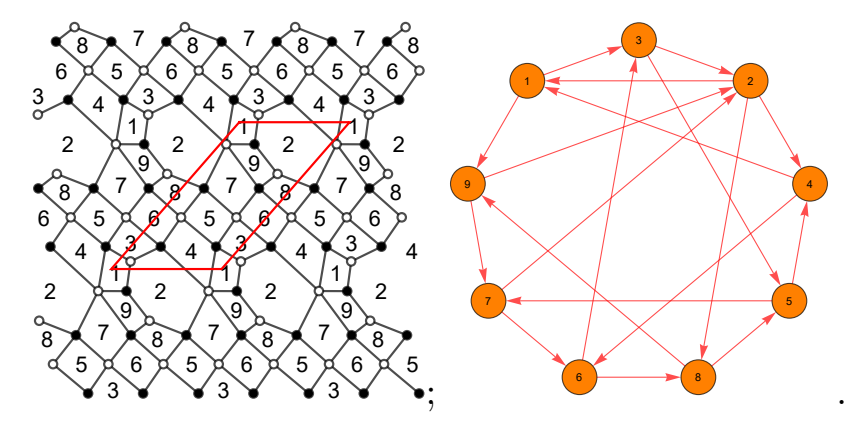
The superpotential is

$$
\begin{aligned}
W= & X_{13} X_{32} X_{21}+X_{24} X_{41} X_{19} X_{97} X_{72}+X_{35} X_{57} X_{76} X_{63}+X_{46} X_{68} X_{85} X_{54} \\
& +X_{89} X_{92} X_{28}-X_{41} X_{13} X_{35} X_{54}-X_{32} X_{24} X_{46} X_{63}-X_{85} X_{57} X_{72} X_{28} \\
& -X_{76} X_{68} X_{89} X_{97}-X_{92} X_{21} X_{19} .
\end{aligned}
$$

The number of perfect matchings is $c=32$, which leads to gigantic $P, Q_{t}$ and $G_{t}$. Hence, we will not list them here. The GLSM fields associated to each point are shown in (5.128), where

$$
q=\left\{q_{1}, q_{2}\right\}, \quad r=\left\{r_{1}, \ldots, r_{9}\right\}, \quad s=\left\{s_{1}, \ldots, s_{14}\right\}, \quad t=\left\{t_{1}, t_{2}\right\} .
$$

The mesonic symmetry reads $\mathrm{U}(1)^{2} \times \mathrm{U}(1)_{\mathrm{R}}$ and the baryonic symmetry reads $\mathrm{U}(1)_{\mathrm{h}}^{4} \times \mathrm{U}(1)^{4}$, where the subscripts "R" and "h" indicate R- and hidden symmetries respectively.

The Hilbert series of the toric cone is

$$
\begin{aligned}
H S= & \frac{1}{\left(1-\frac{t_{1}}{t_{3}}\right)\left(1-\frac{t_{1}}{t_{2} t_{3}}\right)\left(1-\frac{t_{2} t_{3}^{3}}{t_{1}^{2}}\right)}+\frac{1}{\left(1-\frac{1}{t_{2}}\right)\left(1-\frac{t_{2}}{t_{1}}\right)\left(1-t_{1} t_{3}\right)} \\
& +\frac{1}{\left(1-t_{1}\right)\left(1-\frac{t_{2}}{t_{1}}\right)\left(1-\frac{t_{3}}{t_{2}}\right)}+\frac{1}{\left(1-\frac{1}{t_{1}}\right)\left(1-t_{2}\right)\left(1-\frac{t_{1} t_{3}}{t_{2}}\right)} \\
& +\frac{1}{\left(1-\frac{1}{t_{1}}\right)\left(1-\frac{t_{1}}{t_{2}}\right)\left(1-t_{2} t_{3}\right)}+\frac{1}{\left(1-t_{1}\right)\left(1-\frac{1}{t_{2}}\right)\left(1-\frac{t_{2} t_{3}}{t_{1}}\right)} \\
& +\frac{1}{\left(1-\frac{t_{1}}{t_{3}}\right)\left(1-\frac{t_{3}}{t_{2}}\right)\left(1-\frac{t_{2} t_{3}}{t_{1}}\right)}+\frac{1}{\left(1-t_{2}\right)\left(1-\frac{t_{1}}{t_{2}}\right)\left(1-\frac{t_{3}}{t_{1}}\right)} \\
& +\frac{1}{\left(1-\frac{t_{3}}{t_{1}}\right)\left(1-t_{2} t_{3}\right)\left(1-\frac{t_{1}}{t_{2} t_{3}}\right)} .
\end{aligned}
$$

The volume function is then

$$
V=-\frac{-24 b_{1}+b_{2}^{2}+12 b_{2}-117}{\left(b_{1}+3\right)\left(b_{2}-3\right)\left(b_{2}+3\right)\left(b_{1}-b_{2}+3\right)\left(2 b_{1}-b_{2}-9\right)} .
$$

Minimizing $V$ yields $V_{\min }=(83+13 \sqrt{65}) / 1350$ at $b_{1}=(15 \sqrt{65}-81) / 32, b_{2}=0$. Thus, $a_{\max }=\frac{675}{8192}(13 \sqrt{65}-83)$. Together with the superconformal conditions, we can solve for the R-charges of the bifundamentals. Then the R-charges of GLSM fields should satisfy

$$
\begin{aligned}
& \left(1280 p_{2}+512 p_{4}+768 p_{5}\right) p_{3}^{2}+\left(1280 p_{2}^{2}+1536 p_{4} p_{2}+1536 p_{5} p_{2}-2560 p_{2}+512 p_{4}^{2}\right. \\
& \left.+768 p_{5}^{2}-1024 p_{4}+512 p_{4} p_{5}-1536 p_{5}\right) p_{3}=-768 p_{4} p_{2}^{2}-512 p_{5} p_{2}^{2}-768 p_{4}^{2} p_{2}-512 p_{5}^{2} p_{2} \\
& +1536 p_{4} p_{2}-512 p_{4} p_{5} p_{2}+1024 p_{5} p_{2}-256 p_{4} p_{5}^{2}-256 p_{4}^{2} p_{5}+512 p_{4} p_{5}-325 \sqrt{65}+2075
\end{aligned}
$$

constrained by $\sum_{i=1}^{5} p_{i}=2$ and $0<p_{i}<2$, with others vanishing. 


\section{Five hexagons}

\subsection{Polytope 41: $\mathrm{PdP}_{4 e}(3)$}

The polytope is

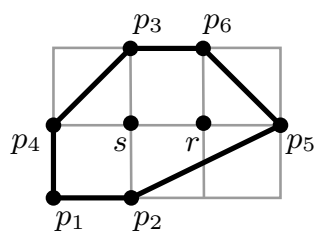

The brane tiling and the corrresponding quiver are
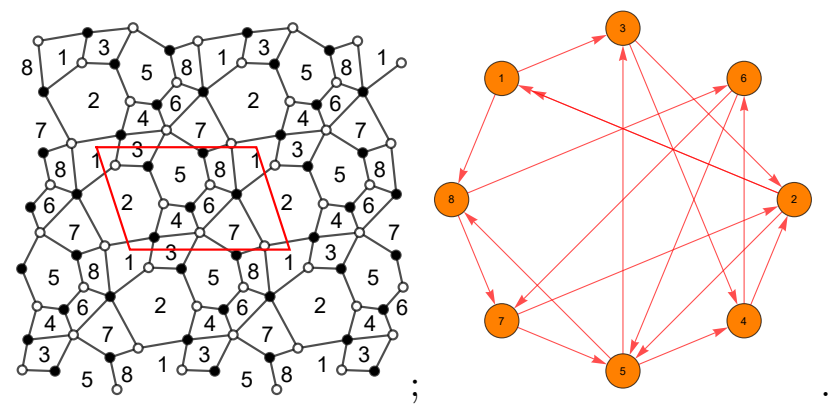

The superpotential is

$$
\begin{aligned}
W= & X_{72} X_{21}^{1} X_{18} X_{87}+X_{13} X_{32} X_{21}^{2}+X_{25} X_{54} X_{42}+X_{46} X_{67} X_{75} X_{53} X_{34}+X_{58} X_{86} X_{65} \\
& -X_{86} X_{67} X_{72} X_{21}^{2} X_{18}-X_{21}^{1} X_{13} X_{34} X_{42}-X_{32} X_{25} X_{53}-X_{54} X_{46} X_{65} \\
& -X_{75} X_{58} X_{87}
\end{aligned}
$$

The perfect matching matrix is

$$
P=\left(\begin{array}{c|ccccccccccccccccccccccccc} 
& r_{1} & r_{2} & s_{1} & s_{2} & s_{3} & s_{4} & p_{1} & p_{2} & r_{3} & r_{4} & r_{5} & s_{5} & s_{6} & p_{3} & r_{6} & s_{7} & p_{4} & p_{5} & p_{6} & r_{7} & s_{8} & r_{8} & s_{9} & s_{10} & s_{11} \\
\hline X_{13} & 1 & 0 & 0 & 0 & 1 & 0 & 0 & 1 & 0 & 0 & 1 & 1 & 0 & 0 & 1 & 0 & 0 & 0 & 0 & 1 & 0 & 1 & 0 & 0 & 1 \\
X_{18} & 1 & 0 & 1 & 1 & 1 & 0 & 0 & 0 & 0 & 0 & 0 & 1 & 0 & 1 & 0 & 0 & 0 & 0 & 0 & 0 & 0 & 0 & 0 & 0 & 0 \\
X_{21}^{1} & 0 & 1 & 0 & 0 & 0 & 1 & 1 & 0 & 0 & 0 & 0 & 0 & 1 & 0 & 0 & 0 & 1 & 0 & 0 & 0 & 0 & 0 & 0 & 0 & 0 \\
X_{21}^{2} & 0 & 1 & 0 & 0 & 0 & 1 & 0 & 0 & 0 & 0 & 0 & 0 & 1 & 0 & 0 & 0 & 0 & 1 & 1 & 0 & 0 & 0 & 0 & 0 & 0 \\
X_{25} & 1 & 1 & 0 & 0 & 0 & 0 & 0 & 1 & 0 & 0 & 0 & 1 & 1 & 0 & 1 & 0 & 0 & 1 & 0 & 1 & 0 & 1 & 0 & 0 & 0 \\
X_{32} & 0 & 0 & 1 & 1 & 0 & 0 & 1 & 0 & 1 & 1 & 0 & 0 & 0 & 1 & 0 & 1 & 1 & 0 & 0 & 0 & 1 & 0 & 1 & 1 & 0 \\
X_{34} & 0 & 0 & 0 & 1 & 0 & 0 & 0 & 0 & 0 & 1 & 0 & 0 & 0 & 0 & 0 & 0 & 0 & 1 & 0 & 0 & 0 & 0 & 0 & 1 & 0 \\
X_{42} & 0 & 0 & 1 & 0 & 0 & 0 & 0 & 0 & 1 & 0 & 0 & 0 & 0 & 1 & 0 & 1 & 0 & 0 & 1 & 0 & 1 & 0 & 1 & 0 & 0 \\
X_{46} & 1 & 1 & 1 & 0 & 0 & 0 & 0 & 1 & 1 & 0 & 0 & 0 & 0 & 0 & 0 & 0 & 0 & 0 & 0 & 0 & 0 & 1 & 1 & 0 & 0 \\
X_{53} & 0 & 0 & 0 & 0 & 1 & 1 & 0 & 0 & 0 & 0 & 1 & 0 & 0 & 0 & 0 & 0 & 0 & 0 & 1 & 0 & 0 & 0 & 0 & 0 & 1 \\
X_{54} & 0 & 0 & 0 & 1 & 1 & 1 & 1 & 0 & 0 & 1 & 1 & 0 & 0 & 0 & 0 & 0 & 1 & 0 & 0 & 0 & 0 & 0 & 0 & 1 & 1 \\
X_{58} & 1 & 1 & 1 & 1 & 1 & 1 & 1 & 1 & 1 & 1 & 1 & 0 & 0 & 0 & 0 & 0 & 0 & 0 & 0 & 0 & 0 & 0 & 0 & 0 & 0 \\
X_{65} & 0 & 0 & 0 & 0 & 0 & 0 & 0 & 0 & 0 & 0 & 0 & 1 & 1 & 1 & 1 & 1 & 0 & 1 & 1 & 1 & 1 & 0 & 0 & 0 & 0 \\
X_{67} & 0 & 0 & 0 & 0 & 0 & 0 & 1 & 0 & 0 & 0 & 0 & 0 & 0 & 0 & 0 & 0 & 0 & 0 & 0 & 1 & 1 & 0 & 0 & 0 & 0 \\
X_{72} & 0 & 0 & 0 & 0 & 0 & 0 & 0 & 1 & 1 & 1 & 1 & 0 & 0 & 0 & 1 & 1 & 0 & 0 & 0 & 0 & 0 & 0 & 0 & 0 & 0 \\
X_{75} & 0 & 0 & 0 & 0 & 0 & 0 & 0 & 0 & 0 & 0 & 0 & 1 & 1 & 1 & 1 & 1 & 1 & 0 & 0 & 0 & 0 & 0 & 0 & 0 & 0 \\
X_{86} & 0 & 0 & 0 & 0 & 0 & 0 & 0 & 0 & 0 & 0 & 0 & 0 & 0 & 0 & 0 & 0 & 1 & 0 & 0 & 0 & 0 & 1 & 1 & 1 & 1 \\
X_{87} & 0 & 0 & 0 & 0 & 0 & 0 & 0 & 0 & 0 & 0 & 0 & 0 & 0 & 0 & 0 & 0 & 0 & 1 & 1 & 1 & 1 & 1 & 1 & 1 & 1
\end{array}\right),
$$


where the relations between bifundamentals and GLSM fields can be directly read off. Then we can get the total charge matrix:

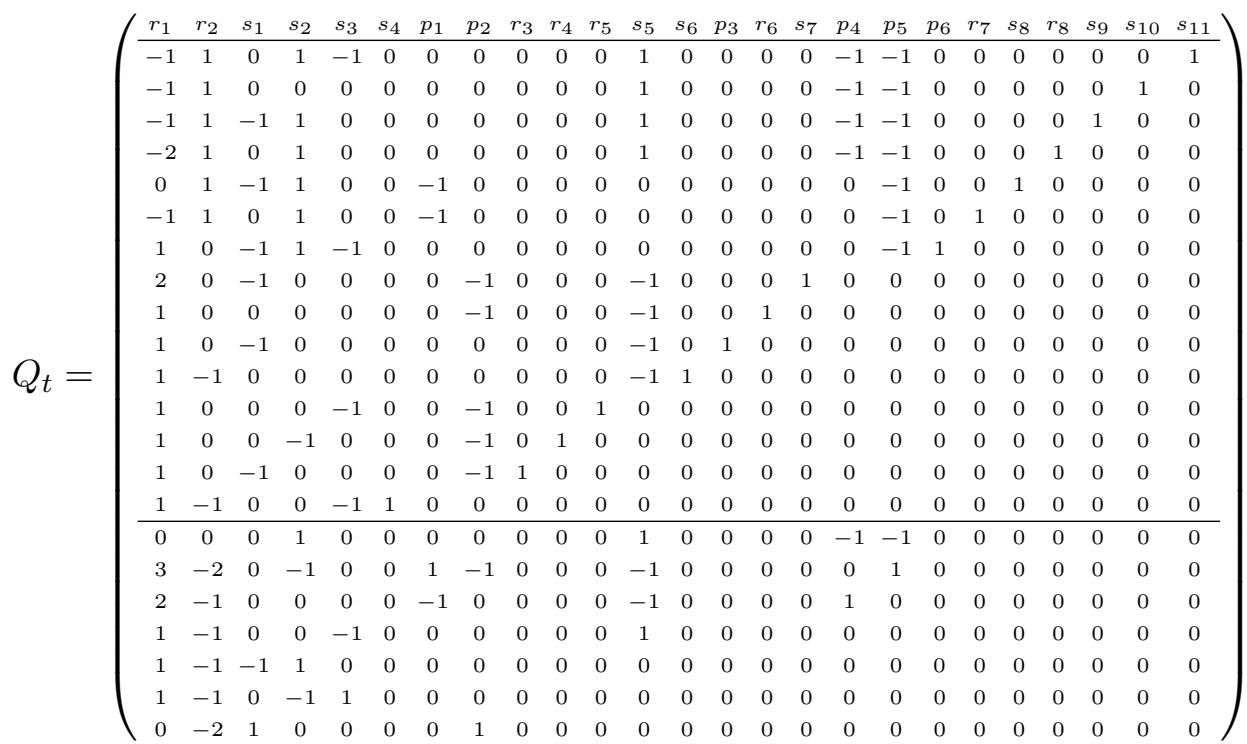

with kernel

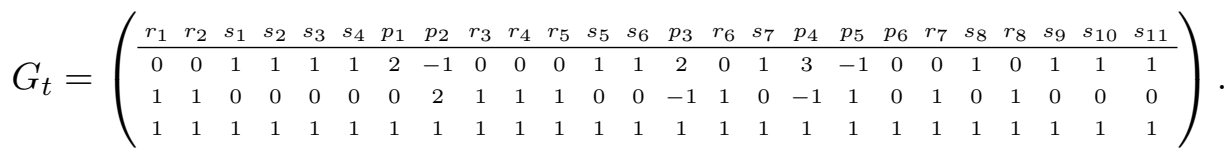

From $G_{t}$, we can get the GLSM fields associated to each point as shown in (6.1), where

$$
r=\left\{r_{1}, \ldots, r_{7}\right\}, \quad s=\left\{s_{1}, \ldots, s_{11}\right\} .
$$

From $Q_{t}$ (and $Q_{F}$ ), the mesonic symmetry reads $\mathrm{U}(1)^{2} \times \mathrm{U}(1)_{\mathrm{R}}$ and the baryonic symmetry reads $\mathrm{U}(1)_{\mathrm{h}}^{4} \times \mathrm{U}(1)^{3}$, where the subscripts " $\mathrm{R}$ " and " $\mathrm{h}$ " indicate $\mathrm{R}$ - and hidden symmetries respectively.

The Hilbert series of the toric cone is

$$
\begin{aligned}
H S= & \frac{1}{\left(1-\frac{1}{t_{2}}\right)\left(1-\frac{t_{1}}{t_{2} t_{3}}\right)\left(1-\frac{t_{2}^{2} t_{3}^{2}}{t_{1}}\right)}+\frac{1}{\left(1-t_{2}\right)\left(1-\frac{t_{1}}{t_{3}}\right)\left(1-\frac{t_{3}^{2}}{t_{1} t_{2}}\right)} \\
& +\frac{1}{\left(1-\frac{1}{t_{2}}\right)\left(1-\frac{t_{2}}{t_{1}}\right)\left(1-t_{1} t_{3}\right)}+\frac{1}{\left(1-t_{1}\right)\left(1-\frac{t_{2}}{t_{1}}\right)\left(1-\frac{t_{3}}{t_{2}}\right)} \\
& +\frac{1}{\left(1-\frac{1}{t_{1}}\right)\left(1-t_{2}\right)\left(1-\frac{t_{1} t_{3}}{t_{2}}\right)}+\frac{1}{\left(1-\frac{1}{t_{1}}\right)\left(1-\frac{t_{1}}{t_{2}}\right)\left(1-t_{2} t_{3}\right)} \\
& +\frac{1}{\left(1-t_{1}\right)\left(1-\frac{1}{t_{2}}\right)\left(1-\frac{t_{2} t_{3}}{t_{1}}\right)}+\frac{1}{\left(1-t_{2}\right)\left(1-\frac{t_{1}}{t_{2}}\right)\left(1-\frac{t_{3}}{t_{1}}\right)} .
\end{aligned}
$$

The volume function is then

$$
V=\frac{6 b_{1}{ }^{2}-b_{1}\left(6 b_{2}+-4 b_{2}{ }^{2}+72\right)-2 b_{2}{ }^{3}+27 b_{2}{ }^{2}+36 b_{2}-513}{\left(b_{1}+3\right)\left(b_{2}-3\right)\left(b_{2}+3\right)\left(b_{1}-b_{2}+3\right)\left(b_{1}+b_{2}-6\right)\left(b_{1}-2\left(b_{2}+3\right)\right)} .
$$


Minimizing $V$ yields $V_{\min }=0.160827$ at $b_{1}=0.979128, b_{2}=0$. Thus, $a_{\max }=1.554465$. Together with the superconformal conditions, we can solve for the R-charges of the bifundamentals. Then the R-charges of GLSM fields should satisfy

$$
\begin{aligned}
& p_{2}\left(3.375 p_{3} p_{6}+1.125 p_{4} p_{6}+4.5 p_{5} p_{6}+1.6875 p_{3}^{2}+0.5625 p_{4}^{2}+1.6875 p_{5}^{2}-3.375 p_{3}\right. \\
& \left.+1.125 p_{3} p_{4}-1.125 p_{4}+3.375 p_{3} p_{5}+1.125 p_{4} p_{5}-3.375 p_{5}+2.25 p_{6}^{2}-4.5 p_{6}\right)+p_{2}^{2}\left(1.6875 p_{3}\right. \\
& \left.+0.5625 p_{4}+1.6875 p_{5}+2.25 p_{6}\right)=-1.125 p_{3} p_{6}^{2}-1.125 p_{4} p_{6}^{2}-1.125 p_{5} p_{6}^{2}-1.125 p_{3}^{2} p_{6} \\
& -1.125 p_{4}^{2} p_{6}-1.125 p_{5}^{2} p_{6}+2.25 p_{3} p_{6}-2.25 p_{3} p_{4} p_{6}+2.25 p_{4} p_{6}-3.375 p_{3} p_{5} p_{6} \\
& -2.25 p_{4} p_{5} p_{6}+2.25 p_{5} p_{6}-0.5625 p_{3} p_{4}^{2}-1.6875 p_{3} p_{5}^{2}-1.125 p_{4} p_{5}^{2}-0.5625 p_{3}^{2} p_{4} \\
& +1.125 p_{3} p_{4}-1.6875 p_{3}^{2} p_{5}-1.125 p_{4}^{2} p_{5}+3.375 p_{3} p_{5}-2.25 p_{3} p_{4} p_{5}+2.25 p_{4} p_{5}-1.03631
\end{aligned}
$$

constrained by $\sum_{i=1}^{6} p_{i}=2$ and $0<p_{i}<2$, with others vanishing.

\subsection{Polytope 42: $\mathrm{PdP}_{5 c}(3)$}

The polytope is

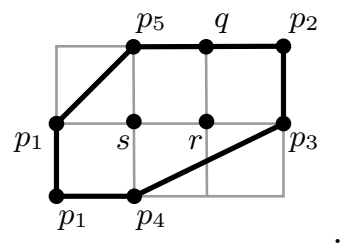

The brane tiling and the corrresponding quiver are
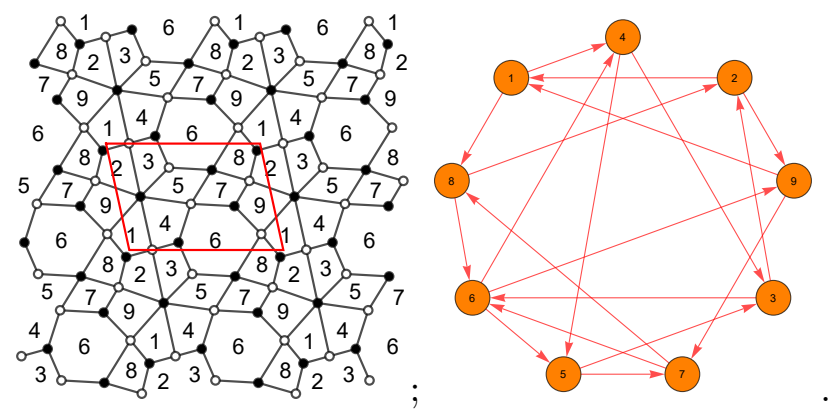

The superpotential is

$$
\begin{aligned}
W= & X_{21} X_{14} X_{43} X_{32}+X_{36} X_{65} X_{53}+X_{45} X_{57} X_{76} X_{64}+X_{69} X_{91} X_{18} X_{86} \\
& +X_{78} X_{82} X_{29} X_{97}-X_{18} X_{82} X_{21}-X_{29} X_{91} X_{14} X_{45} X_{53} X_{32}-X_{43} X_{36} X_{64} \\
& -X_{65} X_{57} X_{78} X_{86}-X_{76} X_{69} X_{97}
\end{aligned}
$$


The perfect matching matrix is

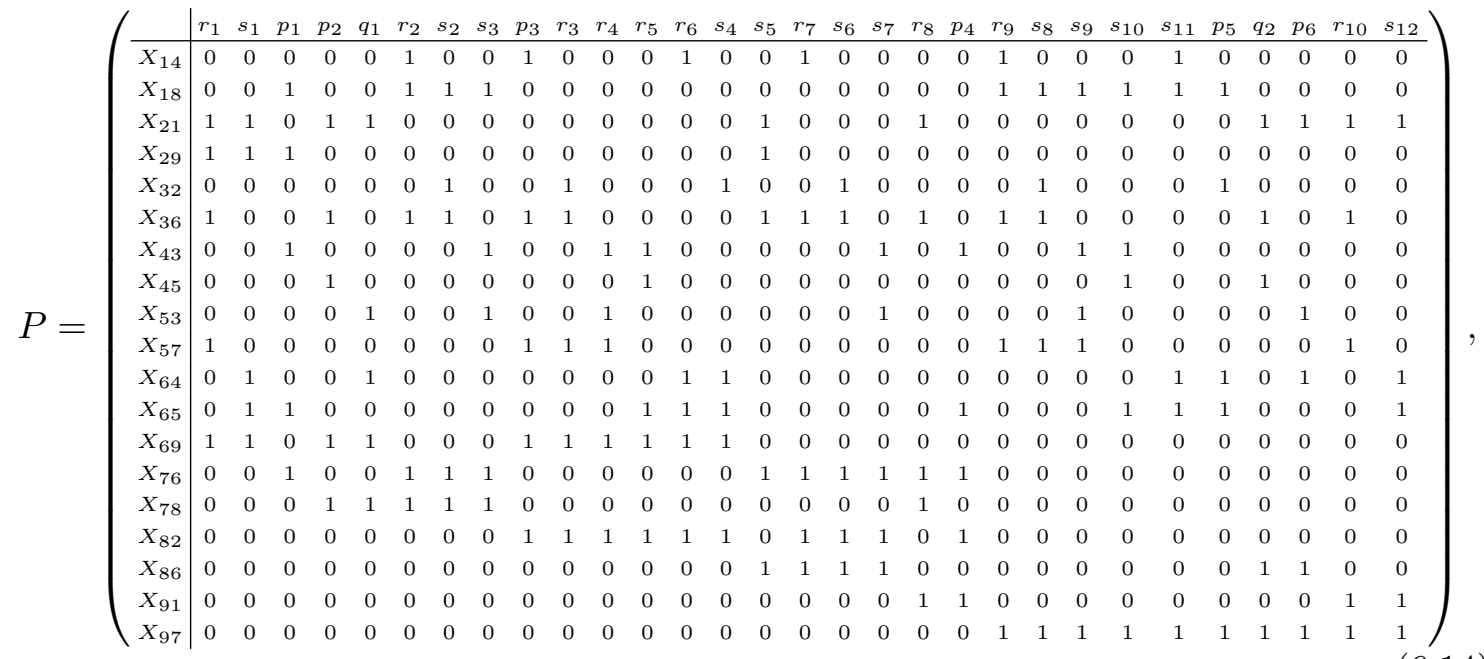

where the relations between bifundamentals and GLSM fields can be directly read off. Then we can get the total charge matrix:

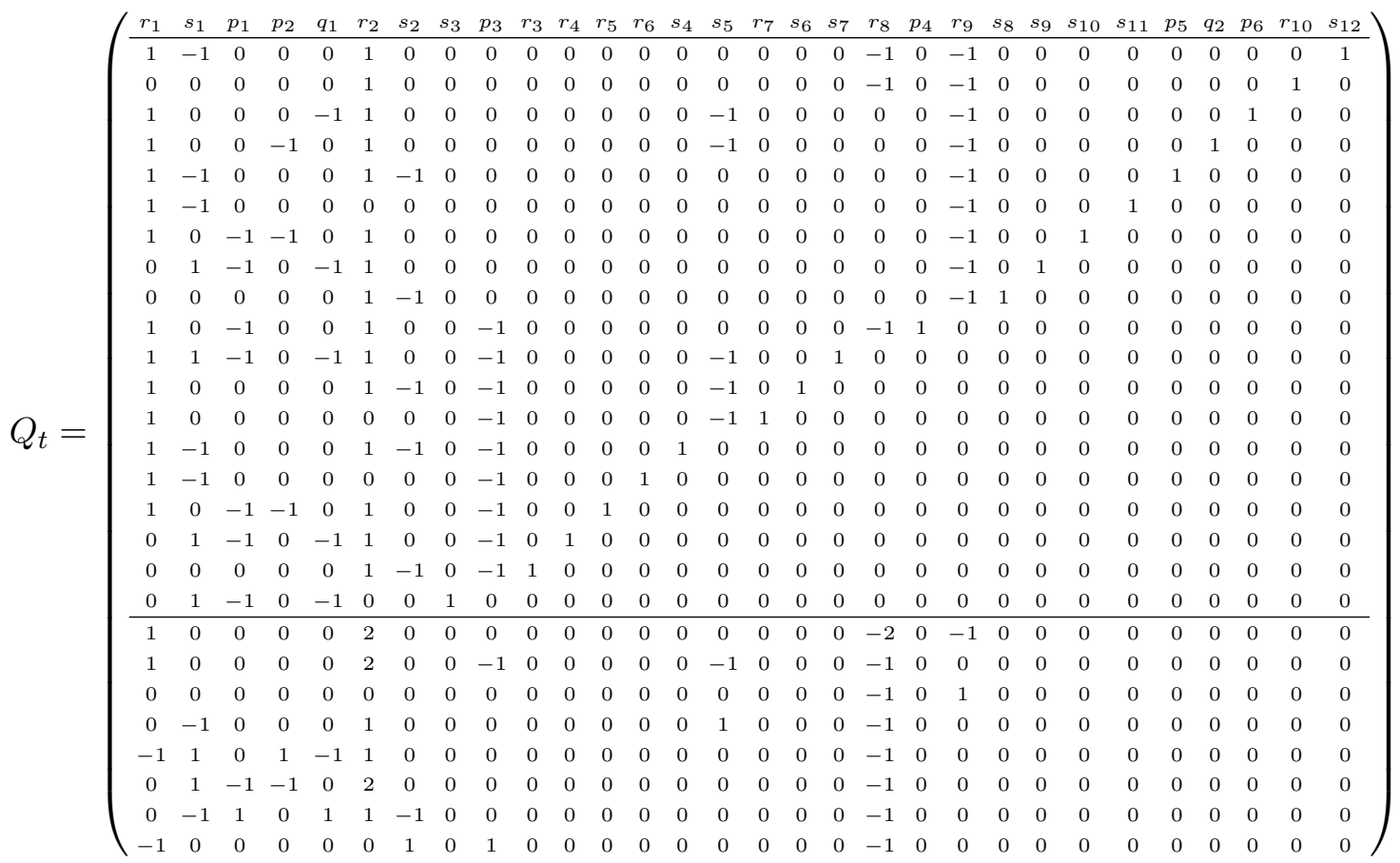

with kernel

$$
G_{t}=\left(\begin{array}{cccccccccccccccccccccccccccccc}
r_{1} & s_{1} & p_{1} & p_{2} & q_{1} & r_{2} & s_{2} & s_{3} & p_{3} & r_{3} & r_{4} & r_{5} & r_{6} & s_{4} & s_{5} & r_{7} & s_{6} & s_{7} & r_{8} & p_{4} & r_{9} & s_{8} & s_{9} & s_{10} & s_{11} & p_{5} & q_{2} & p_{6} & r_{10} & s_{12} \\
\hline 0 & 1 & 3 & -2 & -1 & 0 & 1 & 1 & -1 & 0 & 0 & 0 & 0 & 1 & 1 & 0 & 1 & 1 & 0 & 2 & 0 & 1 & 1 & 1 & 1 & 2 & -1 & 0 & 0 & 1 \\
1 & 0 & -1 & 2 & 1 & 1 & 0 & 0 & 2 & 1 & 1 & 1 & 1 & 0 & 0 & 1 & 0 & 0 & 1 & 0 & 1 & 0 & 0 & 0 & 0 & -1 & 1 & 0 & 1 & 0 \\
1 & 1 & 1 & 1 & 1 & 1 & 1 & 1 & 1 & 1 & 1 & 1 & 1 & 1 & 1 & 1 & 1 & 1 & 1 & 1 & 1 & 1 & 1 & 1 & 1 & 1 & 1 & 1 & 1 & 1
\end{array}\right) \text {. }
$$

From $G_{t}$, we can get the GLSM fields associated to each point as shown in (6.11), where

$$
q=\left\{q_{1}, q_{2}\right\}, \quad r=\left\{r_{1}, \ldots, r_{10}\right\}, \quad s=\left\{s_{1}, \ldots, s_{12}\right\} .
$$


From $Q_{t}$ (and $Q_{F}$ ), the mesonic symmetry reads $\mathrm{U}(1)^{2} \times \mathrm{U}(1)_{\mathrm{R}}$ and the baryonic symmetry reads $\mathrm{U}(1)_{\mathrm{h}}^{4} \times \mathrm{U}(1)^{4}$, where the subscripts " $\mathrm{R}$ " and "h" indicate $\mathrm{R}$ - and hidden symmetries respectively.

The Hilbert series of the toric cone is

$$
\begin{aligned}
H S= & \frac{1}{\left(1-\frac{1}{t_{2}}\right)\left(1-\frac{t_{1}}{t_{2} t_{3}}\right)\left(1-\frac{t_{2}^{2} t_{3}^{2}}{t_{1}}\right)}+\frac{1}{\left(1-t_{2}\right)\left(1-\frac{t_{3}^{2}}{t_{1}}\right)\left(1-\frac{t_{1}}{t_{2} t_{3}}\right)} \\
& +\frac{1}{\left(1-\frac{1}{t_{2}}\right)\left(1-\frac{t_{2}}{t_{3}}\right)\left(1-t_{1} t_{3}\right)}+\frac{1}{\left(1-t_{1}\right)\left(1-\frac{t_{2}}{t_{1}}\right)\left(1-\frac{t_{3}}{t_{2}}\right)} \\
& +\frac{1}{\left(1-\frac{1}{t_{1}}\right)\left(1-t_{2}\right)\left(1-\frac{t_{1} t_{3}}{t_{2}}\right)}+\frac{1}{\left(1-\frac{1}{t_{1}}\right)\left(1-\frac{t_{1}}{t_{2}}\right)\left(1-t_{2} t_{3}\right)} \\
& +\frac{1}{\left(1-t_{1}\right)\left(1-\frac{1}{t_{2}}\right)\left(1-\frac{t_{2} t_{3}}{t_{1}}\right)}+\frac{\left.1-\frac{t_{1}}{t_{3}}\right)\left(1-\frac{t_{3}}{t_{2}}\right)\left(1-\frac{t_{2} t_{3}}{t_{1}}\right)}{\left(1-t_{2}\right)\left(1-\frac{t_{1}}{t_{2}}\right)\left(1-\frac{t_{3}}{t_{1}}\right)} .
\end{aligned}
$$

The volume function is then

$$
V=\frac{b_{1}^{2}\left(\left(b_{2}+9\right)\right)-18 b_{1}\left(b_{2}+3\right)+18\left(b_{2}^{2}-2 b_{2}-27\right)}{\left(b_{1}-6\right)\left(b_{1}+3\right)\left(b_{2}-3\right)\left(b_{2}+3\right)\left(b_{1}-b_{2}+3\right)\left(b_{1}-2\left(b_{2}+3\right)\right)} .
$$

Minimizing $V$ yields $V_{\min }=0.145643$ at $b_{1}=1.383054, b_{2}=0.258873$. Thus, $a_{\max }=$ 1.716526. Together with the superconformal conditions, we can solve for the R-charges of the bifundamentals. Then the R-charges of GLSM fields should satisfy

$$
\begin{aligned}
& \left(1.26563 p_{2}+0.421875 p_{4}+1.26563 p_{5}+2.10938 p_{6}\right) p_{3}^{2}+\left(1.26563 p_{2}^{2}+1.6875 p_{4} p_{2}\right. \\
& +2.53125 p_{5} p_{2}+4.21875 p_{6} p_{2}-2.53125 p_{2}+0.421875 p_{4}^{2}+1.26563 p_{5}^{2}+2.10938 p_{6}^{2} \\
& \left.-0.84375 p_{4}+0.84375 p_{4} p_{5}-2.53125 p_{5}+1.6875 p_{4} p_{6}+2.53125 p_{5} p_{6}-4.21875 p_{6}\right) p_{3} \\
& =-0.84375 p_{4} p_{2}^{2}-1.26563 p_{5} p_{2}^{2}-1.6875 p_{6} p_{2}^{2}-0.84375 p_{4}^{2} p_{2}-1.26563 p_{5}^{2} p_{2}-1.6875 p_{6}^{2} p_{2} \\
& +1.6875 p_{4} p_{2}-0.84375 p_{4} p_{5} p_{2}+2.53125 p_{5} p_{2}-1.6875 p_{4} p_{6} p_{2}-2.53125 p_{5} p_{6} p_{2}+3.375 p_{6} p_{2} \\
& -0.421875 p_{4} p_{5}^{2}-0.84375 p_{4} p_{6}^{2}-0.421875 p_{5} p_{6}^{2}-0.421875 p_{4}^{2} p_{5}+0.84375 p_{4} p_{5} \\
& -0.84375 p_{4}^{2} p_{6}-0.421875 p_{5}^{2} p_{6}+1.6875 p_{4} p_{6}-0.84375 p_{4} p_{5} p_{6}+0.84375 p_{5} p_{6}-0.858263
\end{aligned}
$$

constrained by $\sum_{i=1}^{6} p_{i}=2$ and $0<p_{i}<2$, with others vanishing.

\subsection{Polytope 43: $\mathrm{PdP}_{6 b}(3)$}

The polytope is

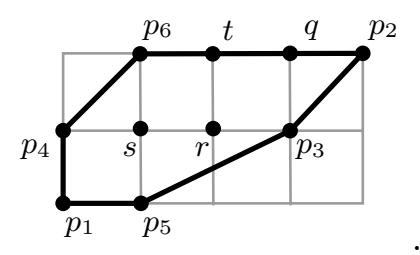


The brane tiling and the corrresponding quiver are
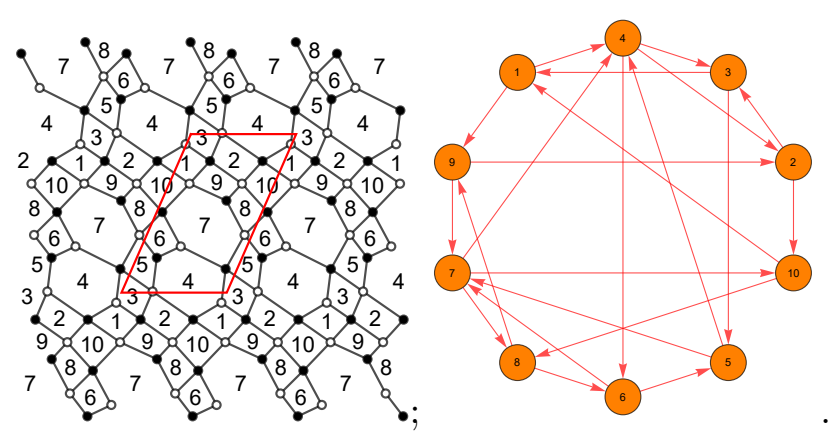

The superpotential is

$$
\begin{aligned}
W= & X_{14} X_{43} X_{31}+X_{23} X_{35} X_{54} X_{42}+X_{46} X_{67} X_{74}+X_{57} X_{78} X_{86} X_{65} \\
& \times X_{7,10} X_{10,1} X_{19} X_{97}+X_{92} X_{2,10} X_{10,8} X_{89}-X_{19} X_{92} X_{23} X_{31}-X_{2,10} X_{10,1} X_{1,4} X_{4,2} \\
& \times X_{43} X_{35} X_{57} X_{74}-X_{54} X_{46} X_{65}-X_{67} X_{7,10} X_{10,8} X_{86}-X_{78} X_{89} X_{97} .
\end{aligned}
$$

The number of perfect matchings is $c=46$, which leads to gigantic $P, Q_{t}$ and $G_{t}$. Hence, we will not list them here. The GLSM fields associated to each point are shown in (6.21), where

$$
q=\left\{q_{1}, \ldots, q_{3}\right\}, r=\left\{r_{1}, \ldots, r_{18}\right\}, s=\left\{s_{1}, \ldots, s_{16}\right\}, t=\left\{t_{1}, \ldots, t_{3}\right\} .
$$

The mesonic symmetry reads $\mathrm{U}(1)^{2} \times \mathrm{U}(1)_{\mathrm{R}}$ and the baryonic symmetry reads $\mathrm{U}(1)_{\mathrm{h}}^{4} \times \mathrm{U}(1)^{5}$, where the subscripts "R" and "h" indicate R- and hidden symmetries respectively.

The Hilbert series of the toric cone is

$$
\begin{aligned}
H S= & \frac{1}{\left(1-\frac{1}{t_{2}}\right)\left(1-\frac{t_{1}}{t_{2} t_{3}}\right)\left(1-\frac{t_{2}^{2} t_{3}^{2}}{t_{1}}\right)}+\frac{1}{\left(1-t_{2}\right)\left(1-\frac{t_{3}^{2}}{t_{1}}\right)\left(1-\frac{t_{1}}{t_{2} t_{3}}\right)} \\
& +\frac{1}{\left(1-\frac{t_{1}}{t_{3}^{2}}\right)\left(1-\frac{t_{3}}{t_{2}}\right)\left(1-\frac{t_{2} t_{3}^{2}}{t_{1}}\right)}+\frac{1}{\left(1-\frac{1}{t_{2}}\right)\left(1-\frac{t_{2}}{t_{1}}\right)\left(1-t_{1} t_{3}\right)} \\
& +\frac{1}{\left(1-t_{1}\right)\left(1-\frac{t_{2}}{t_{1}}\right)\left(1-\frac{t_{3}}{t_{2}}\right)}+\frac{1}{\left(1-\frac{1}{t_{1}}\right)\left(1-t_{2}\right)\left(1-\frac{t_{1} t_{3}}{t_{2}}\right)} \\
& +\frac{1}{\left(1-\frac{1}{t_{1}}\right)\left(1-\frac{t_{1}}{t_{2}}\right)\left(1-t_{2} t_{3}\right)}+\frac{1}{\left(1-t_{1}\right)\left(1-\frac{1}{t_{2}}\right)\left(1-\frac{t_{2} t_{3}}{t_{1}}\right)} \\
& +\frac{1}{\left(1-\frac{t_{1}}{t_{3}}\right)\left(1-\frac{t_{3}}{t_{2}}\right)\left(1-\frac{t_{2} t_{3}}{t_{1}}\right)}+\frac{\left.1-t_{2}\right)\left(1-\frac{t_{1}}{t_{2}}\right)\left(1-\frac{t_{3}}{t_{1}}\right)}{(1-}
\end{aligned}
$$

The volume function is then

$$
V=\frac{2 b_{1}{ }^{2}\left(b_{2}+6\right)-2 b_{1}\left(2 b_{2}{ }^{2}+15 b_{2}+18\right)+2 b_{2}{ }^{3}+9 b_{2}{ }^{2}-108 b_{2}-459}{\left(b_{1}+3\right)\left(b_{2}-3\right)\left(b_{2}+3\right)\left(b_{1}-b_{2}-6\right)\left(b_{1}-b_{2}+3\right)\left(b_{1}-2\left(b_{2}+3\right)\right)} .
$$

Minimizing $V$ yields $V_{\min }=0.126977$ at $b_{1}=2.020709, b_{2}=0.520709$. Thus, $a_{\max }=$ 1.968861. Together with the superconformal conditions, we can solve for the R-charges of 
the bifundamentals. Then the R-charges of GLSM fields should satisfy

$$
\begin{aligned}
& \left(0.5625 p_{3}+1.125 p_{4}+0.5625 p_{5}+1.6875 p_{6}\right) p_{2}^{2}+\left(0.5625 p_{3}^{2}+1.6875 p_{4} p_{3}+1.125 p_{5} p_{3}\right. \\
& +2.8125 p_{6} p_{3}-1.125 p_{3}+1.125 p_{4}^{2}+0.5625 p_{5}^{2}+1.6875 p_{6}^{2}-2.25 p_{4}+0.5625 p_{4} p_{5}-1.125 p_{5} \\
& \left.+2.25 p_{4} p_{6}+1.125 p_{5} p_{6}-3.375 p_{6}\right) p_{2}=-0.84375 p_{4} p_{3}^{2}-0.28125 p_{5} p_{3}^{2}-1.40625 p_{6} p_{3}^{2} \\
& -0.84375 p_{4}^{2} p_{3}-0.28125 p_{5}^{2} p_{3}-1.40625 p_{6}^{2} p_{3}+1.6875 p_{4} p_{3}-0.5625 p_{4} p_{5} p_{3}+0.5625 p_{5} p_{3} \\
& -1.6875 p_{4} p_{6} p_{3}-1.125 p_{5} p_{6} p_{3}+2.8125 p_{6} p_{3}-0.28125 p_{4} p_{5}^{2}-0.28125 p_{4} p_{6}^{2}-0.5625 p_{5} p_{6}^{2} \\
& -0.28125 p_{4}^{2} p_{5}+0.5625 p_{4} p_{5}-0.28125 p_{4}^{2} p_{6}-0.5625 p_{5}^{2} p_{6}+0.5625 p_{4} p_{6}-0.5625 p_{4} p_{5} p_{6} \\
& +1.125 p_{5} p_{6}-0.656287
\end{aligned}
$$

constrained by $\sum_{i=1}^{6} p_{i}=2$ and $0<p_{i}<2$, with others vanishing.

\subsection{Polytope 44: $\mathrm{PdP}_{4 f}(2)$}

The polytope is

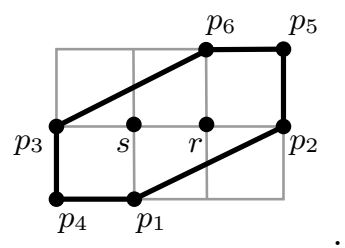

The brane tiling and the corrresponding quiver are

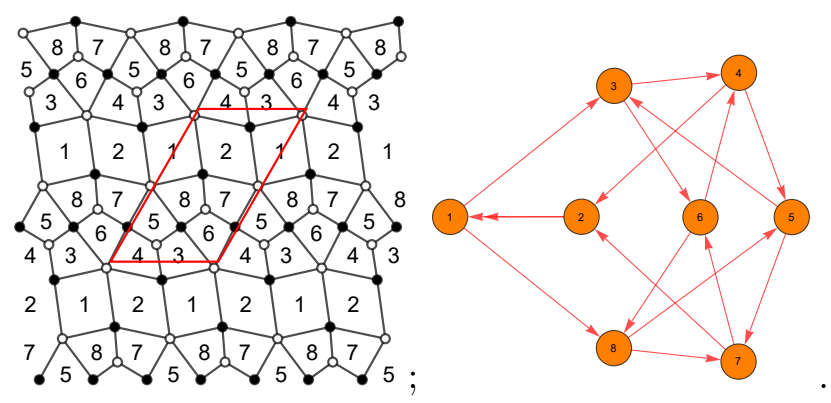

The superpotential is

$$
\begin{aligned}
W= & X_{13} X_{36} X_{64} X_{42} X_{21}^{1}+X_{45} X_{53} X_{34}+X_{68} X_{87} X_{76}+X_{72} X_{21}^{2} X_{18} X_{85} X_{57} \\
& -X_{21}^{2} X_{13} X_{34} X_{42}-X_{53} X_{36} X_{68} X_{85}-X_{64} X_{45} X_{57} X_{76}-X_{87} X_{72} X_{21}^{1} X_{18}
\end{aligned}
$$


The perfect matching matrix is

$$
P=\left(\begin{array}{c|cccccccccccccccccccccccc} 
& r_{1} & r_{2} & s_{1} & p_{1} & s_{2} & p_{2} & r_{3} & r_{4} & s_{3} & s_{4} & s_{5} & p_{3} & r_{5} & r_{6} & s_{6} & p_{4} & p_{5} & r_{7} & r_{8} & s_{7} & p_{6} & r_{9} & s_{8} & s_{9} \\
\hline X_{13} & 1 & 0 & 0 & 0 & 0 & 1 & 0 & 0 & 0 & 1 & 0 & 0 & 0 & 1 & 0 & 0 & 0 & 0 & 1 & 0 & 0 & 1 & 0 & 0 \\
X_{18} & 1 & 0 & 1 & 0 & 1 & 0 & 0 & 0 & 1 & 1 & 0 & 1 & 0 & 0 & 0 & 0 & 0 & 0 & 0 & 0 & 0 & 0 & 0 & 0 \\
X_{21}^{1} & 0 & 1 & 0 & 1 & 0 & 0 & 0 & 0 & 0 & 0 & 1 & 0 & 0 & 0 & 0 & 1 & 0 & 0 & 0 & 0 & 0 & 0 & 0 & 0 \\
X_{21}^{2} & 0 & 1 & 0 & 0 & 0 & 0 & 0 & 0 & 0 & 0 & 1 & 0 & 0 & 0 & 0 & 0 & 1 & 0 & 0 & 0 & 1 & 0 & 0 & 0 \\
X_{34} & 0 & 0 & 0 & 1 & 1 & 0 & 0 & 1 & 1 & 0 & 0 & 0 & 1 & 0 & 0 & 1 & 0 & 1 & 0 & 0 & 0 & 0 & 0 & 1 \\
X_{36} & 0 & 0 & 0 & 0 & 0 & 0 & 0 & 0 & 1 & 0 & 0 & 0 & 1 & 0 & 0 & 0 & 1 & 1 & 0 & 0 & 0 & 0 & 0 & 0 \\
X_{42} & 0 & 0 & 1 & 0 & 0 & 0 & 1 & 0 & 0 & 0 & 0 & 1 & 0 & 0 & 1 & 0 & 0 & 0 & 0 & 1 & 0 & 0 & 1 & 0 \\
X_{45} & 1 & 1 & 1 & 0 & 0 & 1 & 1 & 0 & 0 & 0 & 0 & 0 & 0 & 0 & 0 & 0 & 1 & 0 & 0 & 0 & 0 & 1 & 1 & 0 \\
X_{53} & 0 & 0 & 0 & 0 & 0 & 0 & 0 & 0 & 0 & 1 & 1 & 1 & 0 & 1 & 1 & 0 & 0 & 0 & 1 & 1 & 1 & 0 & 0 & 0 \\
X_{57} & 0 & 0 & 0 & 1 & 0 & 0 & 0 & 0 & 0 & 0 & 0 & 0 & 0 & 0 & 0 & 0 & 0 & 1 & 1 & 1 & 0 & 0 & 0 & 0 \\
X_{64} & 0 & 0 & 0 & 0 & 1 & 0 & 0 & 1 & 0 & 0 & 0 & 0 & 0 & 0 & 0 & 0 & 0 & 0 & 0 & 0 & 1 & 0 & 0 & 1 \\
X_{68} & 1 & 1 & 1 & 1 & 1 & 1 & 1 & 1 & 0 & 0 & 0 & 0 & 0 & 0 & 0 & 0 & 0 & 0 & 0 & 0 & 0 & 0 & 0 & 0 \\
X_{72} & 0 & 0 & 0 & 0 & 0 & 1 & 1 & 1 & 0 & 0 & 0 & 0 & 1 & 1 & 1 & 0 & 0 & 0 & 0 & 0 & 0 & 0 & 0 & 0 \\
X_{76} & 0 & 0 & 0 & 0 & 0 & 0 & 0 & 0 & 1 & 1 & 1 & 1 & 1 & 1 & 1 & 1 & 0 & 0 & 0 & 0 & 0 & 0 & 0 & 0 \\
X_{85} & 0 & 0 & 0 & 0 & 0 & 0 & 0 & 0 & 0 & 0 & 0 & 0 & 0 & 0 & 0 & 1 & 0 & 0 & 0 & 0 & 0 & 1 & 1 & 1 \\
X_{87} & 0 & 0 & 0 & 0 & 0 & 0 & 0 & 0 & 0 & 0 & 0 & 0 & 0 & 0 & 0 & 0 & 1 & 1 & 1 & 1 & 1 & 1 & 1 & 1
\end{array}\right),
$$

where the relations between bifundamentals and GLSM fields can be directly read off. Then we can get the total charge matrix:

$$
Q_{t}=\left(\begin{array}{cccccccccccccccccccccccc}
r_{1} & r_{2} & s_{1} & p_{1} & s_{2} & p_{2} & r_{3} & r_{4} & s_{3} & s_{4} & s_{5} & p_{3} & r_{5} & r_{6} & s_{6} & p_{4} & p_{5} & r_{7} & r_{8} & s_{7} & p_{6} & r_{9} & s_{8} & s_{9} \\
\hline 0 & 1 & 0 & 0 & -1 & 0 & 0 & 0 & 1 & 0 & 0 & 0 & 0 & 0 & 0 & -1 & -1 & 0 & 0 & 0 & 0 & 0 & 0 & 1 \\
0 & 1 & -1 & 0 & 0 & 0 & 0 & 0 & 1 & 0 & 0 & 0 & 0 & 0 & 0 & -1 & -1 & 0 & 0 & 0 & 0 & 0 & 1 & 0 \\
-1 & 1 & 0 & 0 & 0 & 0 & 0 & 0 & 1 & 0 & 0 & 0 & 0 & 0 & 0 & -1 & -1 & 0 & 0 & 0 & 0 & 1 & 0 & 0 \\
1 & 0 & 0 & 0 & -1 & 0 & 0 & 0 & 1 & -1 & 0 & 0 & 0 & 0 & 0 & 0 & -1 & 0 & 0 & 0 & 1 & 0 & 0 & 0 \\
1 & 1 & -1 & -1 & 0 & 0 & 0 & 0 & 1 & -1 & 0 & 0 & 0 & 0 & 0 & 0 & -1 & 0 & 0 & 1 & 0 & 0 & 0 & 0 \\
0 & 1 & 0 & -1 & 0 & 0 & 0 & 0 & 1 & -1 & 0 & 0 & 0 & 0 & 0 & 0 & -1 & 0 & 1 & 0 & 0 & 0 & 0 & 0 \\
0 & 1 & 0 & -1 & 0 & 0 & 0 & 0 & 0 & 0 & 0 & 0 & 0 & 0 & 0 & 0 & -1 & 1 & 0 & 0 & 0 & 0 & 0 & 0 \\
2 & 0 & -1 & 0 & 0 & -1 & 0 & 0 & 0 & -1 & 0 & 0 & 0 & 0 & 1 & 0 & 0 & 0 & 0 & 0 & 0 & 0 & 0 & 0 \\
1 & 0 & 0 & 0 & 0 & -1 & 0 & 0 & 0 & -1 & 0 & 0 & 0 & 1 & 0 & 0 & 0 & 0 & 0 & 0 & 0 & 0 & 0 & 0 \\
1 & 0 & 0 & 0 & 0 & -1 & 0 & 0 & -1 & 0 & 0 & 0 & 1 & 0 & 0 & 0 & 0 & 0 & 0 & 0 & 0 & 0 & 0 & 0 \\
1 & 0 & -1 & 0 & 0 & 0 & 0 & 0 & 0 & -1 & 0 & 1 & 0 & 0 & 0 & 0 & 0 & 0 & 0 & 0 & 0 & 0 & 0 & 0 \\
1 & -1 & 0 & 0 & 0 & 0 & 0 & 0 & 0 & -1 & 1 & 0 & 0 & 0 & 0 & 0 & 0 & 0 & 0 & 0 & 0 & 0 & 0 & 0 \\
1 & 0 & 0 & 0 & -1 & -1 & 0 & 1 & 0 & 0 & 0 & 0 & 0 & 0 & 0 & 0 & 0 & 0 & 0 & 0 & 0 & 0 & 0 & 0 \\
1 & 0 & -1 & 0 & 0 & -1 & 1 & 0 & 0 & 0 & 0 & 0 & 0 & 0 & 0 & 0 & 0 & 0 & 0 & 0 & 0 & 0 & 0 & 0 \\
\hline 1 & 0 & 0 & 0 & 0 & 0 & 0 & 0 & 1 & 0 & 0 & 0 & 0 & 0 & 0 & -1 & -1 & 0 & 0 & 0 & 0 & 0 & 0 & 0 \\
2 & -2 & 0 & 1 & 0 & -1 & 0 & 0 & -1 & 0 & 0 & 0 & 0 & 0 & 0 & 0 & 1 & 0 & 0 & 0 & 0 & 0 & 0 & 0 \\
1 & -1 & 0 & 0 & -1 & 0 & 0 & 0 & 1 & 0 & 0 & 0 & 0 & 0 & 0 & 0 & 0 & 0 & 0 & 0 & 0 & 0 & 0 & 0 \\
2 & -1 & 0 & -1 & 0 & 0 & 0 & 0 & 0 & -1 & 0 & 0 & 0 & 0 & 0 & 1 & 0 & 0 & 0 & 0 & 0 & 0 & 0 & 0 \\
1 & -1 & -1 & 0 & 1 & 0 & 0 & 0 & 0 & 0 & 0 & 0 & 0 & 0 & 0 & 0 & 0 & 0 & 0 & 0 & 0 & 0 & 0 & 0 \\
1 & -1 & 0 & 0 & 0 & 0 & 0 & 0 & -1 & 1 & 0 & 0 & 0 & 0 & 0 & 0 & 0 & 0 & 0 & 0 & 0 & 0 & 0 & 0 \\
0 & -2 & 1 & 0 & 0 & 1 & 0 & 0 & 0 & 0 & 0 & 0 & 0 & 0 & 0 & 0 & 0 & 0 & 0 & 0 & 0 & 0 & 0 & 0
\end{array}\right)
$$

with kernel

$$
G_{t}=\left(\begin{array}{ccccccccccccccccccccccccccc}
r_{1} & r_{2} & s_{1} & p_{1} & s_{2} & p_{2} & r_{3} & r_{4} & s_{3} & s_{4} & s_{5} & p_{3} & r_{5} & r_{6} & s_{6} & p_{4} & p_{5} & r_{7} & r_{8} & s_{7} & p_{6} & r_{9} & s_{8} & s_{9} \\
\hline 0 & 0 & 1 & 1 & 1 & -1 & 0 & 0 & 1 & 1 & 1 & 2 & 0 & 0 & 1 & 2 & -1 & 0 & 0 & 1 & 0 & 0 & 1 & 1 \\
1 & 1 & 0 & 1 & 0 & 2 & 1 & 1 & 0 & 0 & 0 & -1 & 1 & 1 & 0 & 0 & 1 & 1 & 1 & 0 & 0 & 1 & 0 & 0 \\
1 & 1 & 1 & 1 & 1 & 1 & 1 & 1 & 1 & 1 & 1 & 1 & 1 & 1 & 1 & 1 & 1 & 1 & 1 & 1 & 1 & 1 & 1 & 1
\end{array}\right) .
$$

From $G_{t}$, we can get the GLSM fields associated to each point as shown in (6.28), where

$$
r=\left\{r_{1}, \ldots, r_{9}\right\}, \quad s=\left\{s_{1}, \ldots, s_{9}\right\} .
$$

From $Q_{t}$ (and $Q_{F}$ ), the mesonic symmetry reads $\mathrm{U}(1)^{2} \times \mathrm{U}(1)_{\mathrm{R}}$ and the baryonic symmetry reads $\mathrm{U}(1)_{\mathrm{h}}^{4} \times \mathrm{U}(1)^{3}$, where the subscripts " $\mathrm{R}$ " and " $\mathrm{h}$ " indicate $\mathrm{R}$ - and hidden symmetries respectively. 
The Hilbert series of the toric cone is

$$
\begin{aligned}
H S= & \frac{1}{\left(1-\frac{1}{t_{2}}\right)\left(1-\frac{t_{1}}{t_{2} t_{3}}\right)\left(1-\frac{t_{2}^{2} t_{3}^{2}}{t_{1}}\right)}+\frac{1}{\left(1-t_{2}\right)\left(1-\frac{t_{2}}{t_{1}}\right)\left(1-\frac{t_{1} t_{3}}{t_{2}^{2}}\right)} \\
& +\frac{1}{\left(1-t_{2}\right)\left(1-\frac{t_{3}^{2}}{t_{1}}\right)\left(1-\frac{t_{1}}{t_{2} t_{3}}\right)}+\frac{1}{\left(1-\frac{1}{t_{2}}\right)\left(1-\frac{t_{2}}{t_{1}}\right)\left(1-t_{1} t_{3}\right)} \\
& +\frac{1}{\left(1-\frac{1}{t_{1}}\right)\left(1-\frac{t_{1}}{t_{2}}\right)\left(1-t_{2} t_{3}\right)}+\frac{1}{\left(1-t_{1}\right)\left(1-\frac{1}{t_{2}}\right)\left(1-\frac{t_{2} t_{3}}{t_{1}}\right)} \\
& +\frac{1}{\left(1-\frac{t_{1}}{t_{3}}\right)\left(1-\frac{t_{3}}{t_{2}}\right)\left(1-\frac{t_{2} t_{3}}{t_{1}}\right)}+\frac{1}{\left(1-t_{2}\right)\left(1-\frac{t_{1}}{t_{2}}\right)\left(1-\frac{t_{3}}{t_{1}}\right)} .
\end{aligned}
$$

The volume function is then

$$
V=\frac{6\left(b_{1}^{2}-2 b_{1} b_{2}-3 b_{1}+6 b_{2}^{2}+3 b_{2}-99\right)}{\left(b_{1}-6\right)\left(b_{1}+3\right)\left(b_{2}-3\right)\left(b_{2}+3\right)\left(b_{1}-2 b_{2}+3\right)\left(b_{1}-2\left(b_{2}+3\right)\right)} .
$$

Minimizing $V$ yields $V_{\min }=40 / 243$ at $b_{1}=3 / 2, b_{2}=0$. Thus, $a_{\max }=243 / 160$. Together with the superconformal conditions, we can solve for the R-charges of the bifundamentals. Then the R-charges of GLSM fields should satisfy

$$
\begin{aligned}
& \left(15 p_{3}+5 p_{4}+10 p_{5}+15 p_{6}\right) p_{2}^{2}+\left(15 p_{3}^{2}+10 p_{4} p_{3}+30 p_{5} p_{3}+30 p_{6} p_{3}-30 p_{3}+5 p_{4}^{2}+10 p_{5}^{2}\right. \\
& \left.+15 p_{6}^{2}-10 p_{4}+10 p_{4} p_{5}-20 p_{5}+10 p_{4} p_{6}+30 p_{5} p_{6}-30 p_{6}\right) p_{2}=-5 p_{4} p_{3}^{2}-20 p_{5} p_{3}^{2}-15 p_{6} p_{3}^{2} \\
& -5 p_{4}^{2} p_{3}-20 p_{5}^{2} p_{3}-15 p_{6}^{2} p_{3}+10 p_{4} p_{3}-20 p_{4} p_{5} p_{3}+40 p_{5} p_{3}-20 p_{4} p_{6} p_{3}-40 p_{5} p_{6} p_{3} \\
& +30 p_{6} p_{3}-10 p_{4} p_{5}^{2}-10 p_{4} p_{6}^{2}-10 p_{5} p_{6}^{2}-10 p_{4}^{2} p_{5}+20 p_{4} p_{5}-10 p_{4}^{2} p_{6}-10 p_{5}^{2} p_{6}+20 p_{4} p_{6} \\
& -20 p_{4} p_{5} p_{6}+20 p_{5} p_{6}-9
\end{aligned}
$$

constrained by $\sum_{i=1}^{6} p_{i}=2$ and $0<p_{i}<2$, with others vanishing.

\subsection{Polytope 45: $\mathrm{PdP}_{6 c}$ (3)}

The polytope is

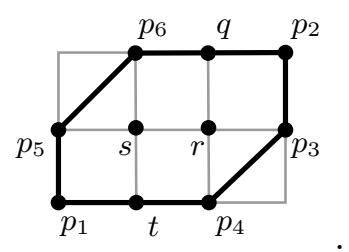

The brane tiling and the corrresponding quiver are
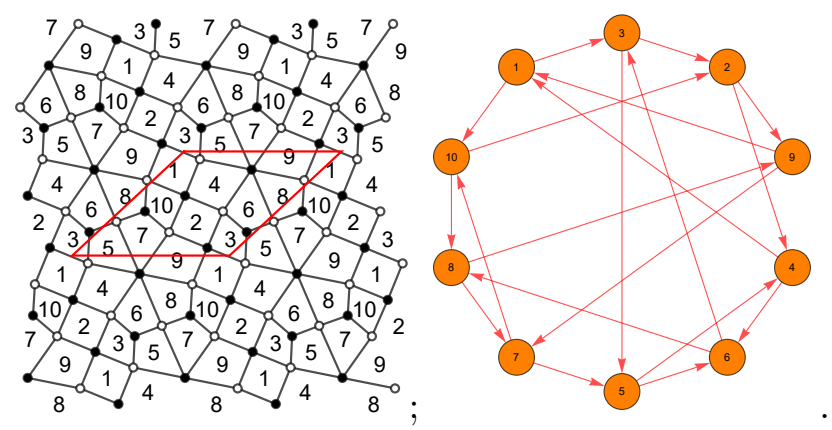
The superpotential is

$$
\begin{aligned}
W= & X_{13} X_{35} X_{54} X_{41}+X_{46} X_{63} X_{32} X_{24}+X_{68} X_{87} X_{75} X_{56}+X_{89} X_{91} X_{1,10} X_{10,8} \\
& +X_{10,2} X_{29} X_{97} X_{7,10}-X_{29} X_{91} X_{13} X_{32}-X_{1,10} X_{10,2} X_{24} X_{41}-X_{63} X_{35} X_{56} \\
& \times X_{54} X_{46} X_{68} X_{89} X_{97} X_{75}-X_{87} X_{7,10} X_{10,8}
\end{aligned}
$$

The number of perfect matchings is $c=40$, which leads to gigantic $P, Q_{t}$ and $G_{t}$. Hence, we will not list them here. The GLSM fields associated to each point are shown in (6.38), where

$$
q=\left\{q_{1}, q_{2}\right\}, \quad r=\left\{r_{1}, \ldots, r_{15}\right\}, \quad s=\left\{s_{1}, \ldots, s_{15}\right\}, \quad t=\left\{t_{1}, t_{2}\right\} .
$$

The mesonic symmetry reads $\mathrm{U}(1)^{2} \times \mathrm{U}(1)_{\mathrm{R}}$ and the baryonic symmetry reads $\mathrm{U}(1)_{\mathrm{h}}^{4} \times \mathrm{U}(1)^{5}$, where the subscripts "R" and "h" indicate R- and hidden symmetries respectively.

The Hilbert series of the toric cone is

$$
\begin{aligned}
H S= & \frac{1}{\left(1-t_{2}\right)\left(1-\frac{t_{3}^{2}}{t_{1}}\right)\left(1-\frac{t_{1}}{t_{2} t_{3}}\right)}+\frac{1}{\left(1-\frac{1}{t_{2}}\right)\left(1-\frac{t_{1}}{t_{3}}\right)\left(1-\frac{t_{2} t_{3}^{2}}{t_{1}}\right)} \\
& +\frac{1}{\left(1-\frac{1}{t_{2}}\right)\left(1-\frac{t_{2}}{t_{1}}\right)\left(1-t_{1} t_{3}\right)}+\frac{1}{\left(1-t_{1}\right)\left(1-\frac{t_{2}}{t_{1}}\right)\left(1-\frac{t_{3}}{t_{2}}\right)} \\
& +\frac{1}{\left(1-\frac{1}{t_{1}}\right)\left(1-t_{2}\right)\left(1-\frac{t_{1} t_{3}}{t_{2}}\right)}+\frac{1}{\left(1-t_{1}\right)\left(1-\frac{1}{t_{1} t_{2}}\right)\left(1-t_{2} t_{3}\right)} \\
& +\frac{1}{\left(1-\frac{1}{t_{1}}\right)\left(1-\frac{t_{1}}{t_{2}}\right)\left(1-t_{2} t_{3}\right)}+\frac{1}{\left(1-\frac{t_{1}}{t_{3}}\right)\left(1-\frac{t_{3}}{t_{2}}\right)\left(1-\frac{t_{2} t_{3}}{t_{1}}\right)} \\
& +\frac{1}{\left(1-t_{2}\right)\left(1-\frac{t_{1}}{t_{2}}\right)\left(1-\frac{t_{3}}{t_{1}}\right)}+\frac{1}{\left(1-\frac{1}{t_{2}}\right)\left(1-t_{1} t_{2}\right)\left(1-\frac{t_{3}}{t_{1}}\right)} .
\end{aligned}
$$

The volume function is then

$$
V=\frac{3\left(4 b_{1}^{2}-4 b_{1}\left(b_{2}+3\right)+3\left(b_{2}^{2}+2 b_{2}-51\right)\right)}{\left(b_{1}-6\right)\left(b_{1}+3\right)\left(b_{2}-3\right)\left(b_{2}+3\right)\left(b_{1}-b_{2}-6\right)\left(b_{1}-b_{2}+3\right)} .
$$

Minimizing $V$ yields $V_{\min }=32 / 243$ at $b_{1}=3 / 2, b_{2}=0$. Thus, $a_{\max }=243 / 128$. Together with the superconformal conditions, we can solve for the R-charges of the bifundamentals. Then the R-charges of GLSM fields should satisfy

$$
\begin{aligned}
& \left(12 p_{3}+16 p_{4}+12 p_{5}+16 p_{6}\right) p_{2}^{2}+\left(12 p_{3}^{2}+32 p_{4} p_{3}+24 p_{5} p_{3}+40 p_{6} p_{3}-24 p_{3}+16 p_{4}^{2}+12 p_{5}^{2}\right. \\
& \left.+16 p_{6}^{2}-32 p_{4}+16 p_{4} p_{5}-24 p_{5}+32 p_{4} p_{6}+24 p_{5} p_{6}-32 p_{6}\right) p_{2}=-8 p_{4} p_{3}^{2}-12 p_{5} p_{3}^{2}-20 p_{6} p_{3}^{2} \\
& -8 p_{4}^{2} p_{3}-12 p_{5}^{2} p_{3}-20 p_{6}^{2} p_{3}+16 p_{4} p_{3}-16 p_{4} p_{5} p_{3}+24 p_{5} p_{3}-32 p_{4} p_{6} p_{3}-24 p_{5} p_{6} p_{3} \\
& +40 p_{6} p_{3}-8 p_{4} p_{5}^{2}-16 p_{4} p_{6}^{2}-4 p_{5} p_{6}^{2}-8 p_{4}^{2} p_{5}+16 p_{4} p_{5}-16 p_{4}^{2} p_{6}-4 p_{5}^{2} p_{6}+32 p_{4} p_{6} \\
& -16 p_{4} p_{5} p_{6}+8 p_{5} p_{6}-9
\end{aligned}
$$

constrained by $\sum_{i=1}^{6} p_{i}=2$ and $0<p_{i}<2$, with others vanishing. 


\section{The toric variety $\widetilde{X(\Delta)}$}

Given a lattice polytope $\Delta$ of (complex) dimension $n$, besides the $(n+1)$-dimensional Calabi-Yau cone which is non-compact, we can also get a compact toric variety $X(\Delta)$ under the construction of inner normal fan $\Sigma(\Delta)$. Here, we give a quick review on the compact toric variety $X(\Delta)$. A detailed treatment can be found in [46, 47].

To build $X(\Delta)$, we choose one interior point as the origin, then the fan $\Sigma(\Delta)$ is constructed out of cones having rays going through the vertices of each face with origin as the apex, viz,

$$
\Sigma(\Delta)=\{\operatorname{pos}(F): F \in \operatorname{Faces}(\Delta)\}
$$

where

$$
\operatorname{pos}(F)=\left\{\sum_{i} \lambda_{i} \boldsymbol{v}_{i}: \boldsymbol{v}_{i} \in F, \lambda_{i} \geq 0\right\}
$$

is the positive hull of the $n$-cone over face $F$. For instance, choosing the left interior point as the origin, the polygon (4.139) in section $4.17, \mathcal{C} /\left(\mathbb{Z}_{3} \times \mathbb{Z}_{2}\right)(1,0,0,2)(0,1,1,0)$, has the toric variety

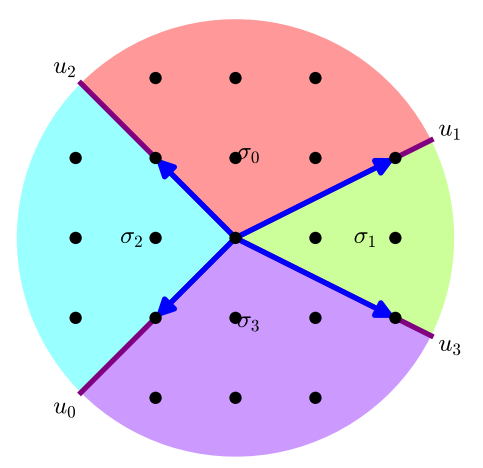

with the cones $\sigma_{i}$ as affine patches.

However, such $X(\Delta)$ may not be smooth. In fact, the toric variety built from (7.3) is not smooth. This is solved by the following definition:

Definition 7.1. The polytope and the corresponding fan are regular if every cone in the fan has generators that form part of a $\mathbb{Z}$-basis.

The regularity can be determined by the determinant of all $n$-tuple vectors of each cone. If all the determinants are \pm 1 , then we have a regular polytope and a regular fan. With regularity, we have [47]

Theorem 7.1. The toric variety $X(\Delta)$ is smooth iff $\Delta$ is regular.

For example, in $(7.3), \operatorname{det}\left(u_{0}, u_{2}\right)=-2$, and therefore the corresponding toric variety is singular. Nevertheless, we can always resolve the singularities via triangulations of the polytope. For reflexive polytopes, FRS triangulations are considered [13, 71], where

- "Fine" stands for all the lattice points of the polytope involved in the triangulation; 
- "Regular" stands for the polytope being regular;

- "Star" stands for the origin being the apex of all the triangulated cones.

Now that we are dealing with polygons having two interior points, $\mathrm{F}$ and $\mathrm{S}$ can not be simultaneously satisfied. Hence, we will drop the condition F, and contemplate RS triangulations. Under such triangulations, we get a complete resolution, $\widetilde{X(\Delta)}$, of $X(\Delta)$. For instance, (7.3) can be resolved to

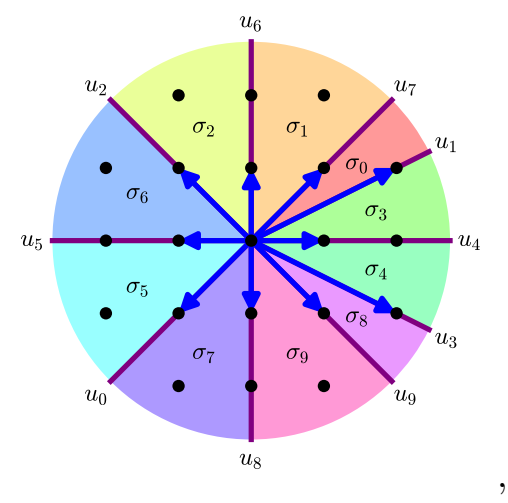

which is complete and smooth.

\subsection{The two interior points as origins}

From [72], we know that $X(\Delta)$ 's constructed from reflexive polytopes are Gorenstein Fano, i.e., its anticanonical divisor $K_{X}$ is Cartier and ample. However, as we have two interior points here, $X(\Delta)$ does not hold this property any more. Actually, since we have two choices of the origin, we can build two compact toric varieties, which may or may not be the same. ${ }^{17}$

For the two $\widetilde{X(\Delta)}$ 's built from $\Delta$ to coincide, it is necessary for them to have the same Euler number. As we will discuss in section 7.2, the Euler number of $\widetilde{X(\Delta)}$ equals to the number of triangles under the triangulation, viz, the number of two-dimensional cones. Hence, this can be checked by counting the numbers of triangles under triangulations. After complete resolutions, we find that there are only 12 polygons that have $\widetilde{X(\Delta)}$ 's with different Euler numbers. In terms of the ordering in appendix A, they are (2), (4), (10), (12), (15), (18), (19), (23), (37), (38), (39) and (40).

As the two interior points is connected by a straight line, now for simplicity, let us call this line the "spine" of the polygon. Since the Euler number is related to triangulation, it is not hard to see that when we have zero or two perimeter points lying on the spine, the two Euler numbers are equal. ${ }^{18}$ On the other hand, if there is only one perimeter point on the spine, the two complete resolutions would yield different Euler numbers. This is because

\footnotetext{
${ }^{17}$ Notice that even though we have this choice on the level of the toric 2 -fold, the affine 3 -fold is the same and hence the gauge theories are the same.

${ }^{18}$ Hence, none of the hexagons belongs to the 12 polygons as it has been proven in [27] that the two interior points of a hexagon must lie on the same diagonal.
} 


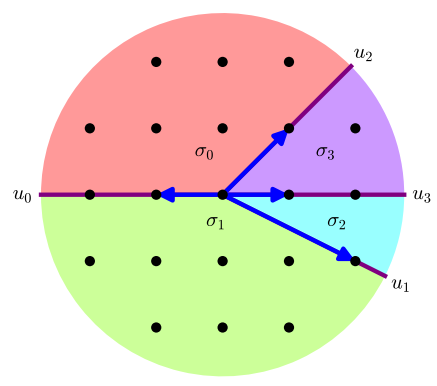

(a)

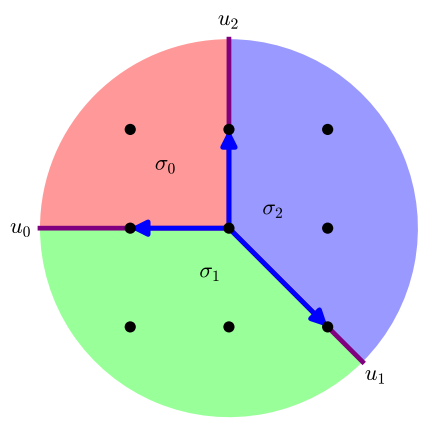

(b)

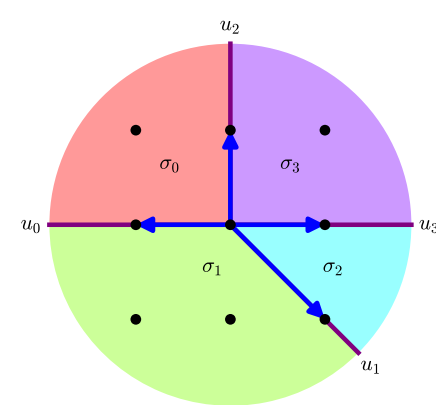

(c)

Figure 1. (a) The complete resolution $\widetilde{X_{0}}$ is constructed from the zeroth-grade point. The Euler number $\chi_{0}$ is 4 . (b) The toric variety $X_{1}$ is already smooth, viz, $X_{1}=\widetilde{X}_{1}$. The Euler number $\chi_{1}$ is 3. (c) We make a further blow-up on $X_{1}$ by adding the ray $u_{3}=(1,0)$. The new variety $\widetilde{X_{1}^{\prime}}$ has Euler number $\chi_{1}^{\prime}=4$.

for these three points on the spine, if the interior point is in the middle (which we will refer to as the "zeroth-grade" point), the fan will have rays extending to both of the other two points on the spine. For the other interior point (which we will refer to as the "first-grade" point), the fan will only have one ray on the spine. Thus, the zeroth-/first-grade Euler numbers will differ by 1 :

$$
\chi_{0}-\chi_{1}=1 .
$$

As will be discussed in section 7.2, the first Chern numbers will then satisfy $C_{1,1}-C_{1,0}=1$ where $C_{1, i}$ denotes the first Chern number of $\widetilde{X_{i}(\Delta)}$ from the $i^{\text {th }}$-grade point. ${ }^{19}$

For the remanining 33 polygons who have two zeroth-grade points, it turns out that not only the corresponding Chern numbers of $\widehat{X(\Delta)}$ 's, but also the two Chern classes (and hence the two Euler numbers) are equal. For the 12 polygons with first-grade points, consider the complete resolution whose fan has the first-grade point as the apex. If we add another ray opposite to the original ray on the spine, i.e., we further resolve the complete smooth surface, then we will reach a new variety with Euler number $\chi_{1}^{\prime}=\chi_{1}+1=\chi_{0}$. As a matter of fact, we find that the total Chern classes of $\widetilde{X_{0}(\Delta)}$ and $\widetilde{X_{1}^{\prime}(\Delta)}$ are equal:

$$
c\left(\widetilde{X_{1}^{\prime}}\right)=c\left(\widetilde{X_{0}}\right) .
$$

As an example, the different resolutions of (3.10) in section 3.2 is depicted in figure 1.

It is worth noting that all the 12 polygons with first-grade points can be higgsed from a minimal parent theory which also has a first-grade point (and two zeroth-grade points). This minimal parent theory is

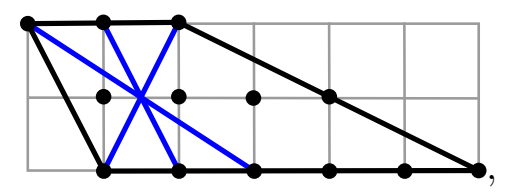

\footnotetext{
${ }^{19}$ For polytopes with arbitrarily many interior points, the zeroth-grade points will be those which give the largest possible Euler number $n$ while the $m^{\text {th }}$-grade points will give Euler number $(n-m)$.
} 


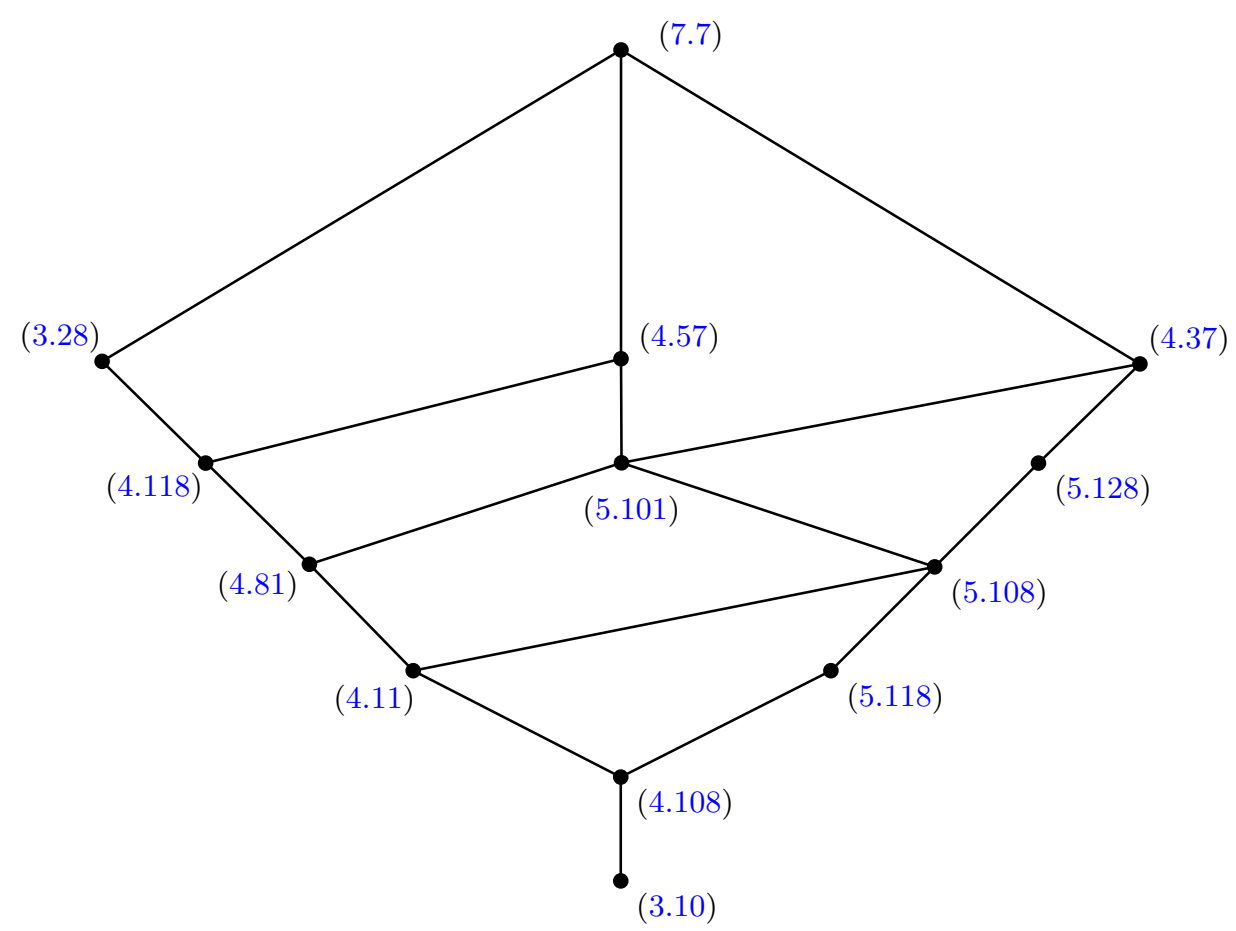

Figure 2. Each point in the Hasse diagram corresponds to a toric diagram, with the corresponding equation number as indicated. Going down along the lines in the Hasse diagram corresponds to the process of higgsing.

where the blue lines indicate three of the higgsed polygons each from blowing down three points. The remaining 9 can be obtained from these three polygons. Notice that the first-grade point in (7.7) is always higgsed away, and one zeroth-grade point becomes a first-grade point after higgsing. Since these polygons form a poset, we can arrange them into a Hasse diagram ${ }^{20}$ as in figure 2.

As the first-grade point trivially yields a different $\widetilde{X_{1}(\Delta)}$ from $\widetilde{X_{0}(\Delta)}$, we will consider $\widetilde{X_{1}^{\prime}(\Delta)}$ which has an extra step of resolution when comparing the two compact smooth complete varieties built from each toric diagram. Since the characteristic classes are always the same for the two varieties, we need a new approach to distinguish them. Our strategy is the same as classifying inequivalent lattice polygons, that is, checking whether the two fans are related by $\operatorname{SL}(2, \mathbb{Z})$ transformations (along with translations and reflections). ${ }^{21}$ One way to see this is to tell whether the vectors ending on the each row/column are properly shifted. Another way is to consider the determinants since all the transformations have determinant \pm 1 and all the $2 \times 2$ matrices with determinant \pm 1 is such a transformation. Then if we pick out any corresponding pairs of vectors from the two fans, the matrices they form should have the same determinant up to a sign.

\footnotetext{
${ }^{20}$ It is worth noting that recently Hasse diagrams has become a powerful tool to study various geometric spaces, along with magnetic quivers, in theories with 8 supercharges. See, for example, [73-78].

${ }^{21}$ More precisely, as the origin is always the apex of the cones, we have no translations here, and thus the transformations lie in $\operatorname{SL}(2, \mathbb{Z}) \times \mathbb{Z}_{2}$.
} 
It turns out that this can be directly read off from the symmetries of the toric diagrams since we only have one spine (which is a result of always having two interior points). Due to the existence of the unique spine, the vectors above and below the spine should be shifted along opposite directions. However, as we are moving from one interior point to the other along the spine, the vectors above and below the spine would always be shifted along the same direction. An example is illustrated in figure 1(a,c).

Hence, reflection or rotation ${ }^{22}$ is necessary to make the two varieties coincide. As a result, the two $\widetilde{X(\Delta)}$ 's are the same iff the lattice polygon (under certain $S L(2, \mathbb{Z})$ transformations) satisfies either of the following two: (1) axially symmetric with respect to the perpendicular bisector of the two interior points; (2) centrosymmetric. ${ }^{23}$ Therefore, only 8 out of the 45 toric diagrams give rise to two same $\widetilde{X}(\Delta)$ 's. In terms of the ordering in appendix A, they are (14), (20), (22), (24), (26), (43), (44) and (45).

Before moving on to the next subsection, let us briefly discuss the smoothness of $X(\Delta)$. Although it is not always the case, some $\Delta$ 's still lead to smooth $X(\Delta)$. There are 9 such polygons. In terms of the ordering in appendix A, they are (2), (6), (7), (8), (18), (25), (26), (41) and (42). In particular, since (2) and (18) (that is, the toric diagrams in (3.10) and (4.108), the bottom two points in figure 2) have both zeroth- and first-grade points, only the first-grade points in both of the cases can give smooth varieties directly. The other 7 toric diagrams can all give rise to two smooth complete surfaces without any further resolutions. It is straightforward that all the perimeter points need to be corner points for $X(\Delta)$ to be smooth. If the toric diagram has a first-grade point as well, then the zeroth-grade point cannot yield a smooth $X(\Delta)$.

\subsection{Minimized volumes and topological quantities}

As we have obtained the volume data of the 45 cases in section $3-6$, we plot $1 / V_{\min }$ against the number of lattice points $N$ in figure 3 .

Now we would like to relate the minimized volume functions of Sasaki-Einstein manifolds to the topological quantities of $\widetilde{X(\Delta)}$ 's. From [46, 47], we have

Theorem 7.2. For the smooth projective variety $\widetilde{X(\Delta)}$ of (complex) dimension $n$, the Betti numbers satisfy

$$
b_{2 k-1}=0, b_{2 k}=\sum_{i=k}^{n}(-1)^{i-k}\left(\begin{array}{l}
i \\
k
\end{array}\right) d_{n-i},
$$

where $k=0,1, \ldots, n$ and $d_{j}$ is the number of $j$-dimensional cones in $\widetilde{\Delta}$. As the Euler number $\chi=\sum_{i=0}^{n}(-1)^{i} b_{i}$, then

$$
\chi=d_{n}
$$

This verifies our statement that the Euler number is the number of triangles under the triangulation used in section 7.1. Then

\footnotetext{
${ }^{22}$ Due to reflection, without loss of generality, rotation can be restricted to inversion, viz, rotation by $\pi$.

${ }^{23}$ These two properties then rule out all the toric diagrams with a first-grade point. Even though we further resolve them to make the Chern classes match, we still cannot have same toric varieties.
} 


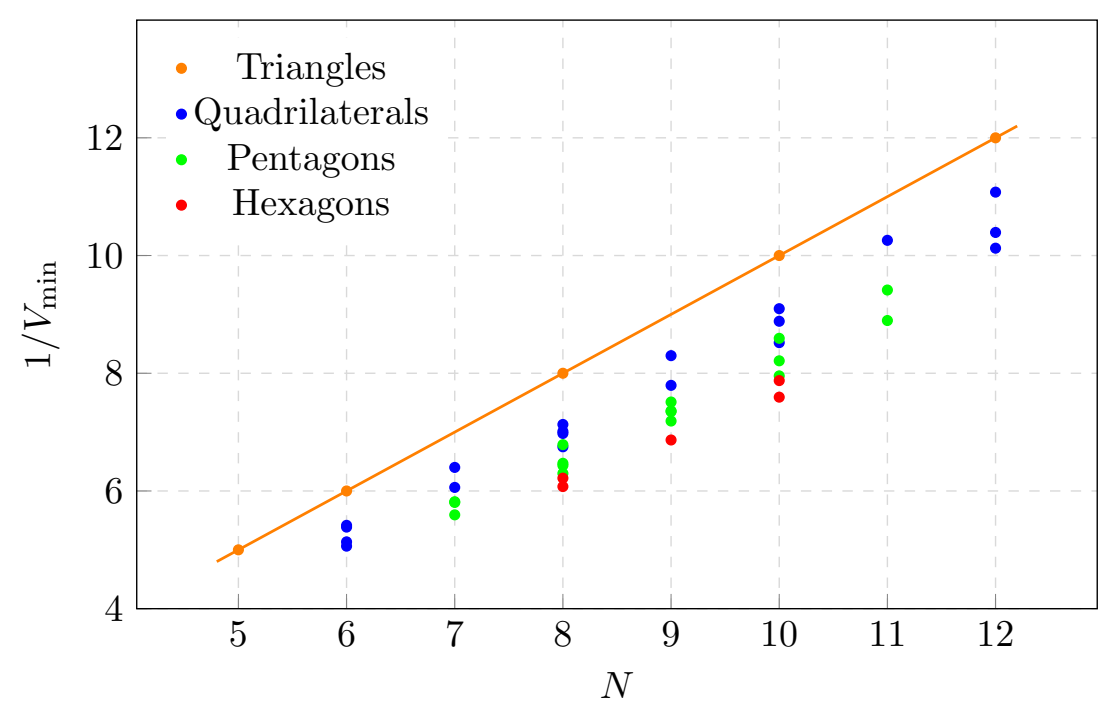

Figure 3. The reciprocals of minimized volumes against the number of lattice points $N$. This is bounded by the straight line $1 / V_{\min }=N$ where the triangles live.

Corollary 7.2.1. For the lattice polygons, we have

$$
b_{0}=b_{4}=1, \quad b_{1}=b_{3}=0, \quad b_{2}=d_{1}-2 d_{0}=d_{1}-2=\chi-2 .
$$

Since $b_{k}=\sum_{i=0}^{k} h^{i, k-i}$, we get

$$
\begin{aligned}
\chi & =\sum_{r, s}(-1)^{r+s} h^{r, s} \\
& =h^{2,2}+h^{2,0}+h^{1,1}+h^{0,2}+h^{0,0} \\
& =2+2 h^{2,0}+h^{1,1} .
\end{aligned}
$$

In fact, we find that the dimension of the Kähler cone over $\widetilde{X(\Delta)}$ is always $\chi-2$. Thus,

$$
h^{2,2}=h^{0,0}=1, \quad h^{2,0}=h^{0,2}=0, \quad h^{1,1}=\chi-2 .
$$

The vanishing $h^{2,0}\left(h^{0,2}\right)$ shows that there is no global sections to the (anti-)canonical bundle. Then the only remaining interesting Hodge number $h^{1,1}$ is determined by the Euler number. As we are now going to see, the (first) Chern number is also determined by the Euler number.

For surfaces, we have two Chern numbers: $C_{1}=\int_{\widetilde{X}} c_{1}^{2}$ and $C_{2}=\int_{\widetilde{X}} c_{2}=\chi$. In figure 4 , we plot $1 / V_{\min }$ against the first and second Chern numbers respectively, following the strategy of [13]. First of all, putting the two graphs together, we can see that the two sets of points are symmetric with respect to $x=6$. Indeed, we find

Proposition 7.3. For a smooth complete toric surface $\widetilde{X}$, we have

$$
C_{1}+\chi=12 .
$$

To prove this, we need the Hirzebruch-Riemann-Roch (HRR) theorem [47]: 
(a)

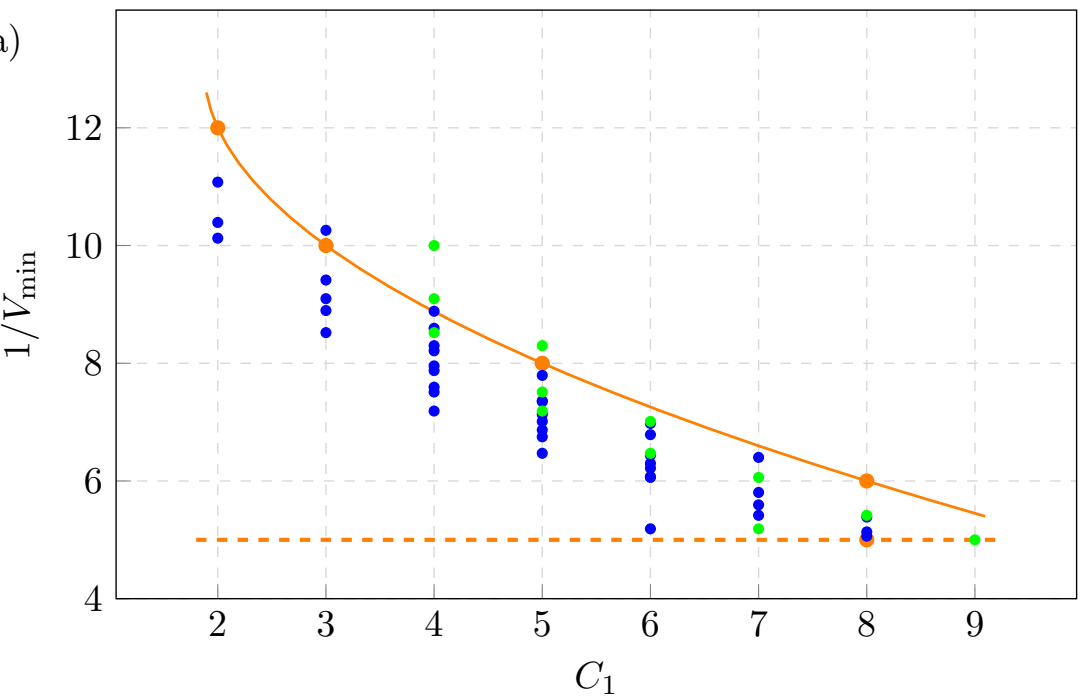

(b)

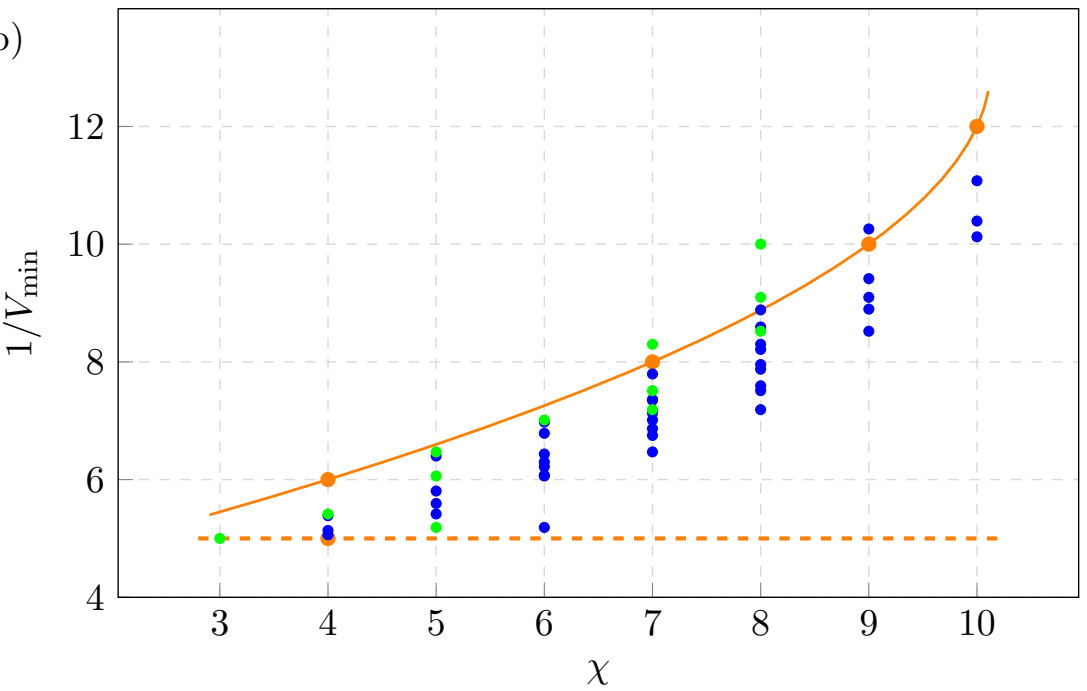

Figure 4. The green points correspond to $\widetilde{X(\Delta)}$ built from first-grade points. The varieties (from zeroth-grade points) of triangles are in orange.

Theorem 7.4. Let $D$ be a divisor of $\widetilde{X}$ and $\mathcal{O}_{\widetilde{X}}(D)$ denote the sheaf of it, then

$$
\chi\left(\mathcal{O}_{\tilde{X}}(D)\right)=\int_{\widetilde{X}} \operatorname{ch}\left(\mathcal{O}_{\tilde{X}}(D)\right) \operatorname{Td}(\tilde{X}) .
$$

Therefore, we are able to prove (7.13):

Proof. Take $D=0$ such that $\operatorname{ch}\left(\mathcal{O}_{\tilde{X}}\right)=1$. Then by HRR theorem,

$$
\begin{aligned}
\chi\left(\mathcal{O}_{\widetilde{X}}\right) & =\int_{\widetilde{X}} \operatorname{Td}(\widetilde{X}) \\
& =\int_{\widetilde{X}}\left(1+\frac{1}{2} c_{1}+\frac{1}{12}\left(c_{1}^{2}+c_{2}\right)\right) \\
& =0+\int_{\widetilde{X}}\left(\frac{1}{12}\left(c_{1}^{2}+c_{2}\right)\right)=\frac{1}{12}\left(C_{1}+\chi\right) .
\end{aligned}
$$


Since $X$ is smooth and complete, by Demazure vanishing [47],

$$
\chi\left(\mathcal{O}_{\widetilde{X}}\right)=\operatorname{dim} H^{0}\left(\widetilde{X}, \mathcal{O}_{\widetilde{X}}\right)-\operatorname{dim} H^{1}\left(\widetilde{X}, \mathcal{O}_{\widetilde{X}}\right)+\operatorname{dim} H^{2}\left(\widetilde{X}, \mathcal{O}_{\widetilde{X}}\right)=1-0+0=1 .
$$

Thus, $C_{1}+\chi=12$.

This would yield many other interesting identities. For instance,

Corollary 7.4.1. For a smooth complete toric surface $\widetilde{X}$, we have

$$
C_{1}-\chi+2=\int_{\widetilde{X}} \operatorname{ch}(\widetilde{X}) \operatorname{Td}(\widetilde{X})
$$

Proof. We start from the r.h.s. :

$$
\begin{aligned}
\int_{\widetilde{X}} \operatorname{ch}(\widetilde{X}) \operatorname{Td}(\widetilde{X}) & =\int_{\widetilde{X}}\left(2+c_{1}+\frac{1}{2} c_{1}^{2}-c_{2}\right)\left(1+\frac{1}{2} c_{1}+\frac{1}{12}\left(c_{1}^{2}+c_{2}\right)\right) \\
& =\frac{7}{6} C_{1}-\frac{5}{6} \chi \\
& =C_{1}+\frac{1}{6} C_{1}-\chi+\frac{1}{6} \chi \\
& =C_{1}-\chi+2,
\end{aligned}
$$

where in the last equality, we have used $C_{1}+\chi=12$.

Henceforth, we will solely plot the graph of minimized volumes with Euler numbers as all the other topological quantities discussed here give no new information.

It is conjectured in [13] that the lower bound of minimized volumes is $1 / \chi$, and the bound is saturated when $\mathcal{X}$ is an abelian orbifold of $\mathbb{C}^{3}$ for reflexive polytopes in any dimensions. However, as we can see from figure $4,1 / V_{\min }$ can be greater than the Euler number. Furthermore, the volumes of triangles do not form a lower bound any more. ${ }^{24}$ There are two cases (13 and 17) that are above the orange curve even if we ignore the green points. Nevertheless, we still find the orange curve seems to follow some pattern. For reflexive cases, such curve would be $\chi=1 / V_{\min }$ as this is the bound mentioned above. For the cases with two interior points, the curve is

$$
\chi=\frac{1}{8}\left(14-\frac{1}{V_{\min }}\right)\left(12-\frac{1}{V_{\min }}\right)+2 .
$$

We suspect that for polygons with arbitrarily many interior points, such curves would follow some specific pattern.

On the other hand, the upper bounds of minimized volumes for reflexive cases in any dimensions are fibrations of $\mathrm{dP}_{3}$ [13]. Here, for polygons with two interior points, we find that the upper bound is $\mathbb{C}^{3} / \mathbb{Z}_{5}(1,2,2)$, which is the only $\mathbb{C}^{3}$ orbifold not on the orange curve.

\footnotetext{
${ }^{24}$ However, we should emphasize that such bound may still be true for reflexive polytopes in any dimension, though we do not have available data to test this.
} 


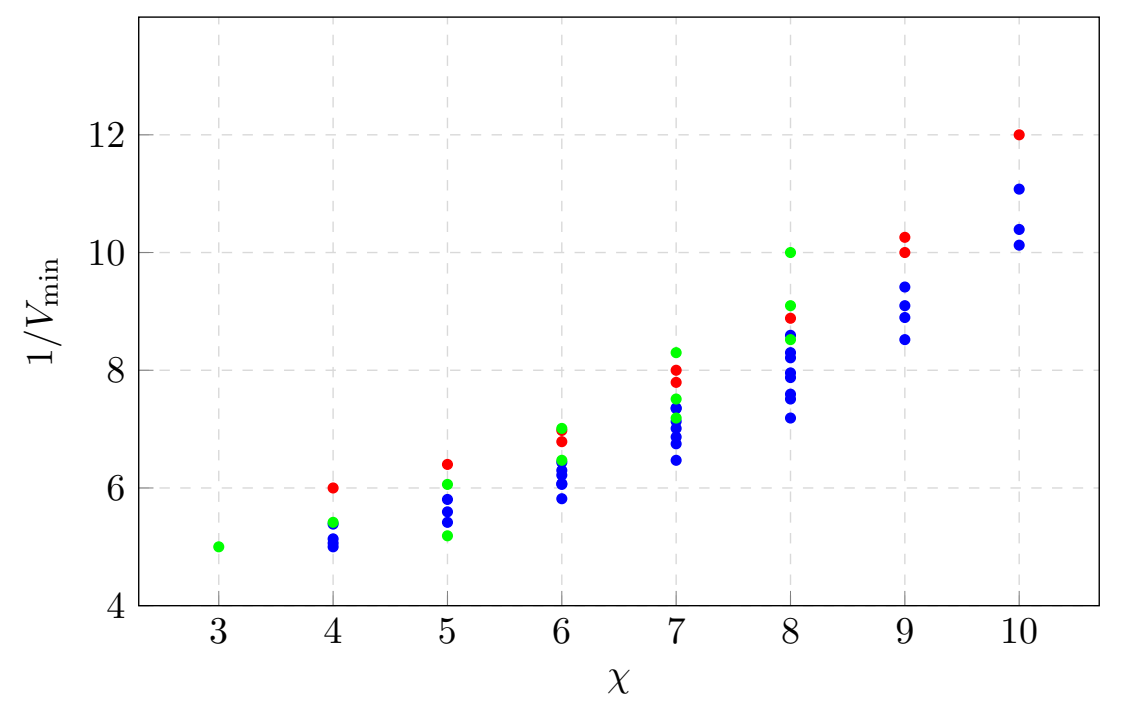

Figure 5. The reciprocal of minimized volumes against the Euler numbers. Here, the points that live much closer to the upper bound in the diagram are labelled red.

It is conjectured in [13] that the bounds of the minimized volumes for toric CY $n$-folds $\mathcal{X}$ with reflexive $(n-1)$-dimensional polytopes as the toric diagrams are

$$
\frac{1}{\chi} \leq V_{\min } \leq m_{n} \int c_{1}^{n-1}
$$

where $m_{3} \sim 3^{-3}, m_{4} \sim 4^{-4}$ and $m_{n}>m_{n+1}$. We have already seen that the first inequality does not hold for non-reflexive cases (while the second one still holds here). In figure 5 , we plot the $\chi-1 / V_{\min }$ diagram again. It is obvious that the area bounded by $1 / V_{\min }=\chi / m_{3}$ and $1 / V_{\min }=(12-\chi) / m_{3}$ is much larger than the region where our data points live. Hence, it is possible that we may extend the above conjecture to

$$
\frac{1}{\chi} \leq V_{\min } / m_{n} \leq \int c_{1}^{n-1}
$$

for non-reflexive polytopes. ${ }^{25}$

As aforementioned, we have two polytopes (13 and 17) that go beyond the bound of $\mathbb{C}^{3}$ orbifolds. ${ }^{26}$ In fact, we find that the red points in figure 5 , including the four $\mathbb{C}^{3}$ orbifolds and (13) and (17), live much closer to the upper bound in the diagram (lower bound of volumes) than to the other points.

Finally, we would also like to know whether the minimized volume of $Y$ with an arbitrary polytope $\Delta$ can be arbitrarily close to 0 , viz, unbounded from above in the $\chi$ $1 / V_{\min }$ diagram. The answer is yes and can be seen from considering the orbifolds. We know that the volume of an orbifold is the volume of its parent divided by the order of the

\footnotetext{
${ }^{25}$ Since the bounded region here is too large, one may consider that we can refine such bounds. However, as $m_{n}$ grows for larger $n$ 's, this might be the best bound for any dimensions. Anyway, the bounds of minimized volumes involving non-reflexive polytopes still require further study.

${ }^{26}$ We will still ignore the green points as they can be turned into non-green points with an extra blow-up.
} 
quotient group, regardless of the action:

$$
\operatorname{vol}(M / \Gamma)=\frac{\operatorname{vol}(M)}{|\Gamma|}
$$

From [62], we know that the volume of a (finite) cone is proportional to the volume of the Sasaki-Einstein manifold. Then the minimized volume function should also follow 27

$$
V(M / \Gamma)=\frac{V(M)}{|\Gamma|} .
$$

For instance, this provides a quick way to see that $V_{\min }\left(\mathbb{C}^{n} / \mathbb{Z}_{n}\right)=1 / n$ as we have shown in section 2.4. For the conifold $\mathcal{C}$, we have $V_{\min }(\mathcal{C})=16 / 27$. Then we would expect the generalized conifolds $(4.21),(4.139)$ and $(4.155)$ to give $V_{\min }(\mathcal{C}) / 3, V_{\min }(\mathcal{C}) / 6$ and $V_{\min }(\mathcal{C}) / 4$ respectively. These are indeed the results we get in section 4 . Also, this does not depend on the orbifold action. The lattice rectangle of size $2 \times 1$ and the toric diagram of $F_{0}$ are both $\mathcal{C}$ quotiented by $\mathbb{Z}_{2}$, but with different actions. However, they both have $V_{\min }=8 / 27$.

\section{Conclusions and outlook}

In this paper, we focused on polygons with two interior points, which serve as the toric diagrams of certain toric $\mathrm{CY}_{3}$ cones, as well as those of compact base surfaces. Using brane tilings, we found the quiver gauge theories associated to D3-branes probing these geometries. The volume functions of Sasaki-Einstein base manifolds were computed so as to get the $\mathrm{R}$-charges of the fields via volume minimization. Compared to reflexive cases, there are much more quivers in the toric phases corresponding to one toric diagram. However, there is always one toric quiver which arises from each orbifold of $\mathbb{C}^{3}$.

We have also analyzed the minimized volumes in terms of the topological quantities of the compact toric varieties constructed from the polygons. To obtain the compact varieties, we made fans over the polytope followed by complete resolutions. However, unlike reflexive cases, we have two choices of origins here, which we called zeroth-grade and first-grade points. It turns out for most of the cases, the Chern numbers and even the Chern classes coincide for the two compact varieties. For those with first-grade points, they obviously do not have such property, but if we further resolve the smooth surface with a ray opposite to the existed ray along the spine, we found that the Chern numbers and classes are again the same for the two varieties. We have also argued that whether the two varieties are the same surface is completely determined by the symmetries of the polygon, namely whether it is axial symmetric or centrosymmetric.

We showed that all the relevant topological invariants, including Chern numbers, Betti numbers and Hodge numbers, are dependent to each other. Hence, all the non-trivial quantities can be expressed with Euler numbers, such as $C_{1}+\chi=12$ and $b_{1}=h^{1,1}=\chi-2$. Thus, we only need to consider the relation between $V_{\min }$ and $\chi$. We plotted the diagram of $1 / V_{\min }$ against $\chi$. It turns out that the volume bounds relation from the reflexive cases does

\footnotetext{
${ }^{27}$ Since it should be clear, we will use the corresponding orbifold to denote the volume function of $Y$ in our notation.
} 
not hold for non-reflexive ones, and we have hinted at a generalized conjecture. Moreover, the minimized volumes of Sasaki-Einstein manifolds of $\mathbb{C}^{3}$ orbifolds do not form a lower bound anymore. However, the upper bound is still safe. Besides, by tracking the orbifold relation, we saw that the volumes coming from any polytopes can only be bounded by 0 , viz, we can have toric diagrams giving as small volumes as we want.

There is still a lot to study for future works. First of all, we have solely considered $2 \mathrm{~d}$ polygons with two interior points. This is quite a strict constraint which only gives us 45 inequivalent toric diagrams. However, as we can see, there are already a sea of toric quivers that we cannot even list all of them in this paper. If we wish to study the gauge theories from polytopes with more interior points and higher dimensions, we cannot search them one by one. For instance, the classification of $3 \mathrm{~d}$ lattice polytopes with two interior points has been done in [79], which gives 22673449 of them up to unimodular equivalence. Instead, a general method needs to be found to get a more detailed understanding of the theories. It would also be interesting to randomize over the space of toric diagrams and try volume-topolgy plots.

Besides, even just for the 45 polygons, not everything is studied. For instance, the specular duality for reflexive polygons is studied in [26]. For reflexive cases, the specular dual of a reflexive toric diagram is still reflexive. Their brane tilings are both on the torus. However, for non-reflexive cases, although the brane tiling is still on the torus, the dual brane tiling is not on $\mathbb{T}^{2}$ anymore. If we go further, there are also cases that neither of the specular duals have brane tiling on $\mathbb{T}^{2}$. We wish to explore these in future.

On the geometry side, the study of compact toric varieties could also be extended to polytopes with more interior points and higher dimensions. We wish to understand minimized volumes via topological invariants for more general cases. In particular, we proposed an enlarged bound for volumes. Whether this is really a bound and whether this is the best bound still requires tests for general cases. However, as it would be impossible to deal with them case by case, new techniques may be necessary.

For reflexive polytopes of dimension $n$, besides the affine $\mathrm{CY}_{n+1}$ cone which is noncompact, we know that compact smooth $\mathrm{CY}_{n-1}$ can be constructed as hypersurfaces in $X(\Delta)$ from [80-85]. However, for non-reflexive ploytopes, we do not have the defining polynomials any more. It would be interesting to study the hypersurfaces for such cases.

\section{Acknowledgments}

We would like to thank Cyril Closset and Alexander Kasprzyk for useful comments. JB would like to thank Zhijie Ji for enjoyable discussions. JB is also grateful to Xiangnan Feng during the work. GBC would like to thank the BC family, in particular, Tracey Colverd for her excellent copyediting and support. YHH would like to thank STFC for grant ST/J00037X/1. 
A The 45 lattice polygons with two interior points

5 Triangles:

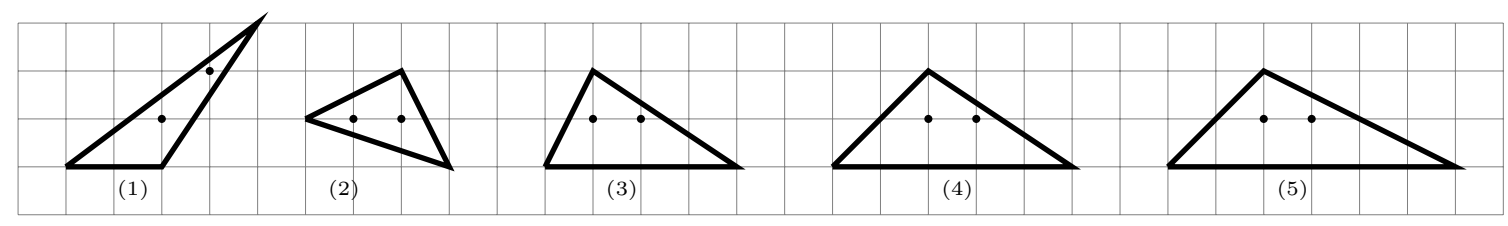

19 Quadrilaterals
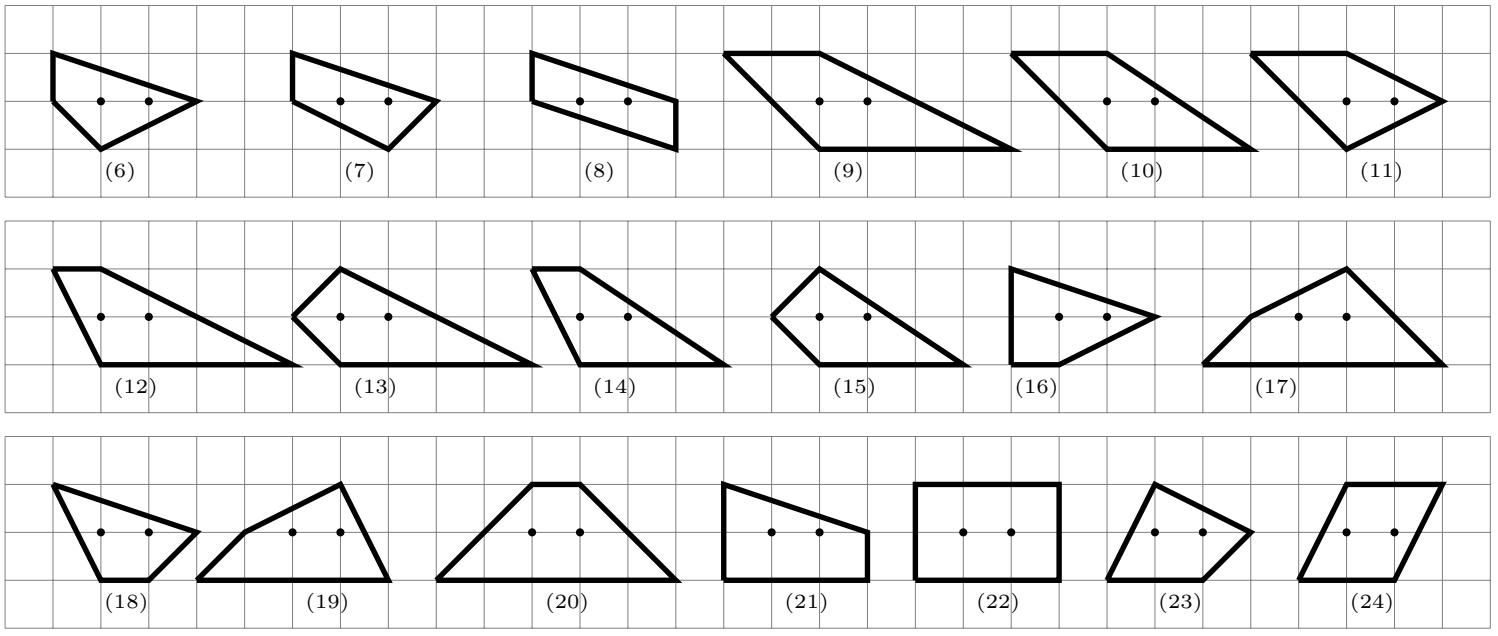

16 Pentagons
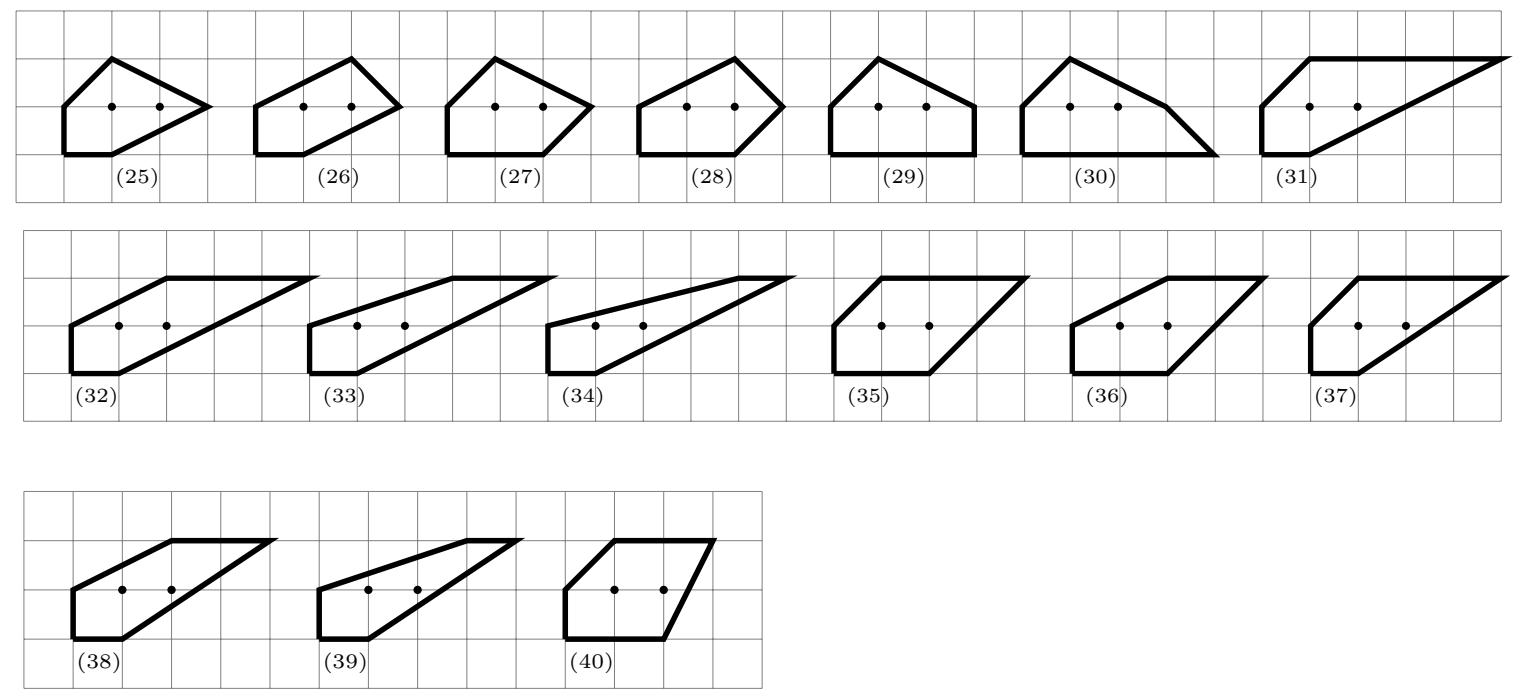


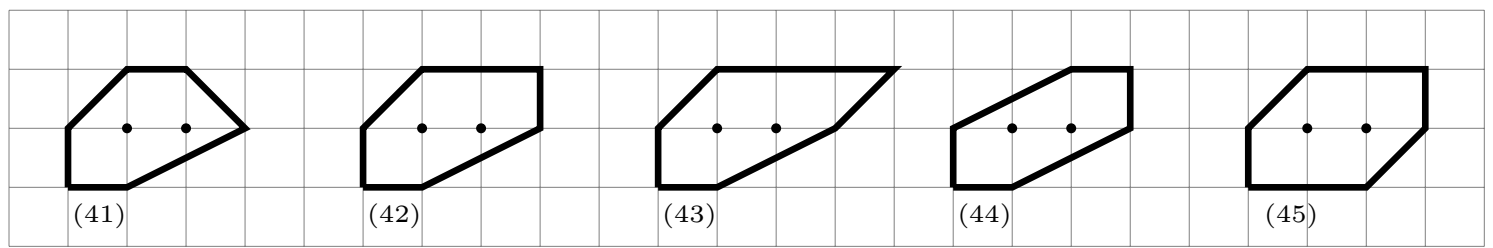

\section{B Volume functions}

For reference, we list all the 45 volume functions and their minima in table 3.

\begin{tabular}{|c|c|c|c|c|c|}
\hline Section & $V$ & $b_{1}^{*}$ & $b_{2}^{*}$ & $V_{\min }$ & {$[L: \mathbb{Q}]$} \\
\hline 3.1 & $\frac{18}{-\frac{18}{\left(b_{2}+3\right)\left(-3 b_{1}+2 b_{2}+6\right)\left(-3 b_{1}+4 b_{2}-6\right)}}$ & $\frac{1}{0}$ & 0 & $\frac{1}{6}$ & 1 \\
\hline 3.2 & $-\frac{\left(b_{2}+3 b_{1}\right)}{\left(b_{1}-2 b_{2}+3\right)\left(2 b_{1}+b_{2}-9\right)\left(b_{1}+3 b_{2}+3\right)}$ & 2 & 0 & $\frac{1}{5}$ & 1 \\
\hline 3.3 & $\frac{16}{\left(b_{2}+3\right)\left(-2 b_{1}+b_{2}-3\right)\left(2 b_{1}+3 b_{2}-9\right)}$ & 2 & -1 & $\frac{1}{8}$ & 1 \\
\hline 3.4 & $\frac{\left(b_{2}+3\right)\left(-2 b_{1}+b_{2}-3\right)\left(2 b_{1}+3 b_{2}-9\right)}{\left(b_{2}+3\right)\left(-b_{1}+b_{2}-3\right)\left(2 b_{1}+3 b_{2}-9\right)}$ & 1 & -1 & $\frac{1}{10}$ & 1 \\
\hline 3.5 & $\frac{6}{\left(b_{2}+3\right)\left(-b_{1}+b_{2}-3\right)\left(b_{1}+2 b_{2}-6\right)}$ & 2 & -1 & $\frac{1}{12}$ & 1 \\
\hline 4.1 & $\frac{3\left(4 b_{1}+2 b_{2}+21\right)}{\left(b_{1}+3\right)\left(b_{1}+b_{2}+3\right)\left(b_{1}+3 b_{2}-6\right)\left(b_{1}-2\left(b_{2}+3\right)\right)}$ & $\frac{1}{2}(-6+3 \sqrt{6})$ & 0 & $\frac{4}{405}(9+4 \sqrt{6})$ & 2 \\
\hline 4.2 & $\frac{6\left(b_{1}+b_{2}+12\right)}{\left(b_{1}+3\right)\left(b_{1}-b_{2}-6\right)\left(b_{1}+2 b_{2}+3\right)\left(b_{1}+3 b_{2}-6\right)}$ & $\frac{3}{2}(\sqrt{33}-5)$ & 0 & $\frac{1}{648}(63+11 \sqrt{33})$ & 2 \\
\hline 4.3 & $\frac{\left(b_{1}+3\right)\left(b_{1}-b_{2}-6\right)\left(b_{1}+2 b_{2}+3\right)\left(b_{1}+3 b_{2}-6\right)}{\left(b_{1}-6\right)\left(b_{1}+3\right)\left(b_{1}+3 b_{2}-6\right)\left(b_{1}+3 b_{2}+3\right)}$ & $\frac{3}{2}$ & 0 & $\frac{16}{81}$ & 1 \\
\hline 4.4 & $-\frac{2\left(b_{2}-9\right)}{\left(b_{2}-3\right)\left(b_{2}+3\right)\left(b_{1}+b_{2}+3\right)\left(b_{1}+2 b_{2}-6\right)}$ & $3(\sqrt{3}-1)$ & $3-2 \sqrt{3}$ & $\frac{\sqrt{3}}{18}$ & 2 \\
\hline 4.5 & $-\frac{2\left(b_{2}-15\right)}{\left(b_{2}-3\right)\left(b_{2}+3\right)\left(b_{1}+b_{2}+3\right)\left(2 b_{1}+3 b_{2}-9\right)}$ & $\frac{1}{2}(5 \sqrt{7}-11)$ & $5+2 \sqrt{7}$ & $\frac{1}{243}(10+7 \sqrt{7})$ & 2 \\
\hline 4.6 & $\frac{2\left(b_{1}+4\left(b_{2}-6\right)\right)}{\left(b_{2}-3\right)\left(b_{1}+b_{2}+3\right)\left(b_{1}+2 b_{2}-6\right)\left(b_{1}-2\left(b_{2}+3\right)\right)}$ & 0 & $4-\sqrt{13}$ & $\frac{1}{648}(46+13 \sqrt{13})$ & 2 \\
\hline 4.7 & $\frac{6\left(b_{2}-5\right)}{\left(b_{2}-3\right)\left(b_{2}+3\right)\left(2 b_{1}+b_{2}+3\right)\left(b_{1}+2 b_{2}-6\right)}$ & $\frac{1}{6}(1+5 \sqrt{13})$ & $\frac{1}{3}(5-2 \sqrt{13})$ & $-\frac{1}{108}(35-13 \sqrt{13})$ & 2 \\
\hline 4.8 & $\frac{2\left(2 b_{1}+b_{2}+15\right)}{\left(b_{2}+3\right)\left(-b_{1}+b_{2}-3\right)\left(b_{1}+b_{2}+3\right)\left(b_{1}+2 b_{2}-6\right)}$ & 3.27464 & -0.831239 & 0.112571 & 4 \\
\hline 4.9 & $-\frac{8\left(b_{2}-6\right)}{\left(b_{2}-3\right)\left(b_{2}+3\right)\left(2 b_{1}+b_{2}+3\right)\left(2 b_{1}+3 b_{2}-9\right)}$ & $\frac{1}{2}(2 \sqrt{7}-1)$ & $2-\sqrt{7}$ & $\frac{4}{243}(-10+7 \sqrt{7})$ & 2 \\
\hline 4.10 & $\frac{2\left(3 b_{1}+2 b_{2}+24\right)}{\left(b_{2}+3\right)\left(-b_{1}+b_{2}-3\right)\left(b_{1}+b_{2}+3\right)\left(2 b_{1}+3 b_{2}-9\right)}$ & 2.19488194 & -0.760489 & 0.142613 & 4 \\
\hline 4.11 & $-\frac{b_{1}-12\left(b_{2}+4\right)}{\left(b_{1}+6\right)\left(b_{2}+3\right)\left(b_{1}-2 b_{2}-3\right)\left(b_{1}+3 b_{2}-3\right)}$ & -2.8546585 & -0.17276 & 0.156243 & 4 \\
\hline 4.12 & $-\frac{5 b_{1}-7 b_{2}+24}{\left(b_{2}+3\right)\left(b_{1}-2 b_{2}+3\right)\left(b_{1}-b_{2}+3\right)\left(b_{1}+b_{2}-6\right)}$ & 1.8379935 & -0.95469 & 0.0974795 & 4 \\
\hline 4.13 & $-\frac{2\left(b_{1}-7 b_{2}-36\right)}{\left(b_{2}+3\right)\left(b_{1}-b_{2}-6\right)\left(2 b_{1}+b_{2}+3\right)\left(b_{1}+3 b_{2}-6\right)}$ & 1.2608787 & -0.21349 & 0.184633 & 4 \\
\hline 4.14 & $-\frac{8 b_{1}-11 b_{2}+39}{\left(b_{2}+3\right)\left(b_{1}-2 b_{2}+3\right)\left(b_{1}-b_{2}+3\right)\left(2 b_{1}+b_{2}-9\right)}$ & 0.8345102 & -0.93610217 & 0.120498 & 4 \\
\hline 4.15 & $-\frac{18-4 b_{2}}{\left(b_{2}-3\right)\left(b_{2}+3\right)\left(-b_{1}+b_{2}-3\right)\left(b_{1}+b_{2}-6\right)}$ & $\frac{3}{2}$ & $\frac{1}{2}(3-\sqrt{21})$ & $\frac{4}{225}(-27+7 \sqrt{21})$ & 2 \\
\hline 4.16 & $-\frac{3\left(b_{1}-15\right)}{\left(b_{1}-6\right)\left(b_{1}+3\right)\left(b_{2}+3\right)\left(b_{1}+3 b_{2}-6\right)}$ & $3(2-\sqrt{3})$ & $\frac{1}{2}(\sqrt{3}-3)$ & $\frac{2}{9 \sqrt{3}}$ & 2 \\
\hline 4.17 & $\frac{18}{\left(b_{1}-6\right)\left(b_{1}+3\right)\left(b_{2}-3\right)\left(b_{2}+3\right)}$ & $\frac{3}{2}$ & 0 & $\frac{8}{81}$ & 1 \\
\hline 4.18 & $-\frac{4 b_{1}-7 b_{2}-69}{\left(b_{2}+3\right)\left(-2 b_{1}+b_{2}-3\right)\left(-b_{1}+b_{2}+6\right)\left(b_{1}+2 b_{2}-6\right)}$ & 1.20148202 & -0.4914321 & 0.165004 & 4 \\
\hline 4.19 & $\frac{48}{\left(b_{2}-3\right)\left(b_{2}+3\right)\left(-2 b_{1}+b_{2}-3\right)\left(-2 b_{1}+b_{2}+9\right)}$ & $\frac{3}{2}$ & 0 & $\frac{4}{27}$ & 1 \\
\hline 5.1 & $-\frac{b_{1}^{2}-2 b_{1}\left(4 b_{2}+15\right)+4\left(b_{2}{ }^{2}-6 b_{2}-45\right)}{\left(b_{1}+3\right)\left(b_{2}+3\right)\left(b_{1}-b_{2}+3\right)\left(b_{1}+2 b_{2}-6\right)\left(b_{1}-2\left(b_{2}+3\right)\right)}$ & 0.746501345 & -0.1982794 & 0.17226 & 11 \\
\hline 5.2 & $-\frac{b_{1} 2-4 b_{1}\left(b_{2}+3\right)+4 b_{2}-30 b_{2}-207}{\left(b_{1}+3\right)\left(b_{2}+3\right)\left(b_{1}-2 b_{2}+3\right)\left(b_{1}+b_{2}-6\right)\left(b_{1}-2\left(b_{2}+3\right)\right)}$ & 1.11941442 & -0.21197378 & 0.178752 & 8 \\
\hline 5.3 & $\begin{array}{c}-\frac{2 b_{1}^{2}-4 b_{1}\left(b_{2}+6\right)+2 b_{2}{ }^{2}-3 b_{2}-171}{\left(b_{1}+3\right)\left(b_{2}+3\right)\left(b_{1}-b_{2}-6\right)\left(b_{1}-b_{2}+3\right)\left(b_{1}+2 b_{2}-6\right)} \\
2\left(b_{1}{ }^{2}-b_{1}\left(b_{2}+3\right)+b_{2}{ }^{2}-3 b_{2}-99\right)\end{array}$ & 0.9337514 & -0.449691462 & 0.15542 & 8 \\
\hline 5.4 & $-\frac{\left(b_{1}+3\right)\left(b_{2}+3\right)\left(b_{1}-2 b_{2}+3\right)\left(b_{1}-b_{2}-6\right)\left(b_{1}+b_{2}-6\right)}{3\left(b_{1}{ }^{2}-6 b_{1}+6\left(b_{2}-9\right)\right)}$ & 1.26614895 & -0.4677020986 & 0.158756 & 3 \\
\hline 5.5 & $-\frac{3\left(b_{1}-6 b_{1}+6\left(b_{2}-9\right)\right)}{\left(b_{1}-6\right)\left(b_{1}+3\right)\left(b_{2}+3\right)\left(b_{1}-b_{2}+3\right)\left(b_{1}+2 b_{2}-6\right)}$ & 1.32269853 & -0.70067002 & 0.136079 & 8 \\
\hline 5.6 & $\begin{array}{c}-\frac{4 b_{1}^{2}+4 b_{1}\left(b_{2}-3\right)-2 b_{2}^{2}+39 b_{2}-153}{\left(b_{1}+3\right)\left(b_{2}+3\right)\left(b_{1}-b_{2}+3\right)\left(b_{1}+b_{2}-6\right)\left(b_{1}+2 b_{2}-6\right)} \\
2\left(b_{2} 2-3 b_{2}-36\right)-3 b_{1}\left(b_{2}+5\right)\end{array}$ & 1.939465 & -0.8789301 & 0.116367 & 3 \\
\hline 5.7 & $-\frac{\left(b_{1}+3\right)\left(b_{2}-3\right)\left(b_{2}+3\right)\left(b_{1}-b_{2}+3\right)\left(b_{1}-2\left(b_{2}+3\right)\right)}{-2 b_{1}\left(b_{2}+6\right)+4 b_{2} 2-90}$ & 2.9071583 & 0.6850367 & 0.106224 & 9 \\
\hline 5.8 & $-\frac{-2 b_{1}\left(b_{2}+6\right)+4 b_{2}{ }^{2}-90}{-\frac{\left.b_{1}+3\right)\left(b_{2}-3\right)\left(b_{2}+3\right)\left(b_{1}-2 b_{2}+3\right)\left(b_{1}-2\left(b_{2}+3\right)\right)}{6\left(b_{2}{ }^{2}+b_{2}-18\right)-b_{1}\left(b_{2}+9\right)}}$ & 3.0926707 & 0.479773042 & 0.121782 & 7 \\
\hline 5.9 & 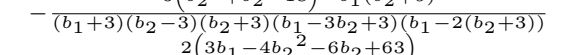 & 2.97485275 & 0.22750743 & 0.135851 & 9 \\
\hline 5.10 & $-\frac{\left.1 b_{1}+3\right)\left(b_{2}-3\right)(b-2+3)\left(b_{1}-4 b_{2}+3\right)\left(b_{1}-2\left(b_{2}+3\right)\right)}{\left(b_{1}-4 b_{2}-00^{2}+03\right.}$ & $\frac{1}{4}(9 \sqrt{57}-57)$ & 0 & $\frac{143+19 \sqrt{57}}{1944}$ & 2 \\
\hline 5.11 & $-\frac{-b_{1}\left(b_{2}+15\right)+b_{2} 2+3 b_{2}-72}{\left(b_{1}+3\right)\left(b_{2}-3\right)\left(b_{2}+3\right)\left(b_{1}-b_{2}-6\right)\left(b_{1}-b_{2}+3\right)}$ & 2.2242667 & 0.26148655 & 0.112411 & 7 \\
\hline 5.12 & $-\frac{2\left(-6 b_{1}+b_{2}^{2}+6 b_{2}-45\right)}{\left(b_{1}+3\right)\left(b_{2}-3\right)\left(b_{2}+3\right)\left(b_{1}-2 b_{2}+3\right)\left(b_{1}-b_{2}-6\right)}$ & $\frac{1}{8}(9 \sqrt{33}-33)$ & 0 & $\frac{1}{972}(59+11 \sqrt{33})$ & 2 \\
\hline 5.13 & $-\frac{3\left(b_{2}{ }^{2}-2 b_{2}-39\right)-4 b_{1}\left(b_{2}+6\right)}{\left(b_{1}+3\right)\left(b_{2}-3\right)\left(b_{2}+3\right)\left(b_{1}-b_{2}+3\right)\left(2 b_{1}-3\left(b_{2}+3\right)\right)}$ & 1.84403082 & 0.57573193 & 0.133134 & 9 \\
\hline 5.14 & $-\frac{6\left(b_{2}{ }^{2}+b_{2}-24\right)-2 b_{1}\left(b_{2}+9\right)}{\left(b_{1}+3\right)\left(b_{2}-3\right)\left(b_{2}+3\right)\left(b_{1}-2 b_{2}+3\right)\left(2 b_{1}-3\left(b_{2}+3\right)\right)}$ & 1.9049613 & 0.28929897 & 0.154554 & 9 \\
\hline
\end{tabular}




\begin{tabular}{|c|c|c|c|c|c|}
\hline $\begin{array}{l}5.15 \\
5.16 \\
\end{array}$ & $\begin{array}{c}\frac{3\left(4 b_{1}-3 b_{2}{ }^{2}-6 b_{2}+57\right)}{\left(b_{1}+3\right)\left(b_{2}-3\right)\left(b_{2}+3\right)\left(b_{1}-3 b_{2}+3\right)\left(2 b_{1}-3\left(b_{2}+3\right)\right)} \\
-\frac{-24 b_{1}+b_{2} 2+12 b_{2}-117}{\left(b_{1}+3\right)\left(b_{2}-3\right)\left(b_{2}+3\right)\left(b_{1}-b_{2}+3\right)\left(2 b_{1}-b_{2}-9\right)}\end{array}$ & $\begin{array}{c}\frac{1}{16}(15 \sqrt{145}-153) \\
\frac{1}{32}(15 \sqrt{65}-81)\end{array}$ & 0 & $\begin{array}{l}\frac{347+29 \sqrt{145}}{4050} \\
\frac{83+13 \sqrt{65}}{1350} \\
\end{array}$ & 2 \\
\hline 6.1 & $\frac{6 b_{1}{ }^{2}-b_{1}\left(-4 b_{2}{ }^{2}+6 b_{2}+72\right)-2 b_{2}{ }^{3}+27 b_{2}{ }^{2}+36 b_{2}-513}{\left(b_{1}+3\right)\left(b_{2}-3\right)\left(b_{2}+3\right)\left(b_{1}-b_{2}+3\right)\left(b_{1}+b_{2}-6\right)\left(b_{1}-2\left(b_{2}+3\right)\right)}$ & 0.97912771 & 0 & 0.160827 & 3 \\
\hline 6.2 & $\frac{b_{1}^{2}\left(\left(b_{2}+9\right)\right)-18 b_{1}\left(b_{2}+3\right)+18\left(b_{2}-2 b_{2}-27\right)}{\left(b_{1}-6\right)\left(b_{1}+3\right)\left(b_{2}-3\right)\left(b_{2}+3\right)\left(b_{1}-b_{2}+3\right)\left(b_{1}-2\left(b_{2}+3\right)\right)}$ & 1.3830544 & 0.2588732 & 0.145643 & 17 \\
\hline 6.3 & $\frac{2 b_{1}^{2}\left(b_{2}+6\right)-2 b_{1}\left(2 b_{2}{ }^{2}+15 b_{2}+18\right)+2 b_{2}{ }^{3}+9 b_{2}{ }^{2}-108 b_{2}-459}{\left(b_{1}+3\right)\left(b_{2}-3\right)\left(b_{2}+3\right)\left(b_{1}-b_{2}-6\right)\left(b_{1}-b_{2}+3\right)\left(b_{1}-2\left(b_{2}+3\right)\right)}$ & 2.02070885 & 0.52070885 & 0.126977 & 3 \\
\hline 6.4 & $\frac{6\left(b_{1}-2 b_{1} b_{2}-3 b 1+6 b_{2}+3 b_{2}-99\right)}{\left(b_{1}-6\right)\left(b_{1}+3\right)\left(b_{2}-3\right)\left(b_{2}+3\right)\left(b_{1}-2 b_{2}+3\right)\left(b_{1}-2\left(b_{2}+3\right)\right)}$ & $\frac{3}{2}$ & 0 & $\frac{40}{243}$ & 1 \\
\hline 6.5 & $\frac{3\left(4 b_{1}{ }^{2}-4 b_{1}\left(b_{2}+3\right)+3\left(b_{2}+2 b_{2}-51\right)\right)}{\left(b_{1}-6\right)\left(b_{1}+3\right)\left(b_{2}-3\right)\left(b_{2}+3\right)\left(b_{1}-b_{2}-6\right)\left(b_{1}-b_{2}+3\right)}$ & $\frac{3}{2}$ & 0 & $\frac{32}{243}$ & 1 \\
\hline
\end{tabular}

Table 3. Volume functions $V$, critical Reeb vectors $b_{i}^{*}$ and their corresponding volume minima $V_{\min }$, with $b_{3}=3$. In the last column, we list the degree of the extension $L$ (of $\mathbb{Q}$ ), where $L=$ $\mathbb{Q}\left(b_{1}^{*}, b_{2}^{*}\right)=\mathbb{Q}\left(b_{1}^{*}\right)$.

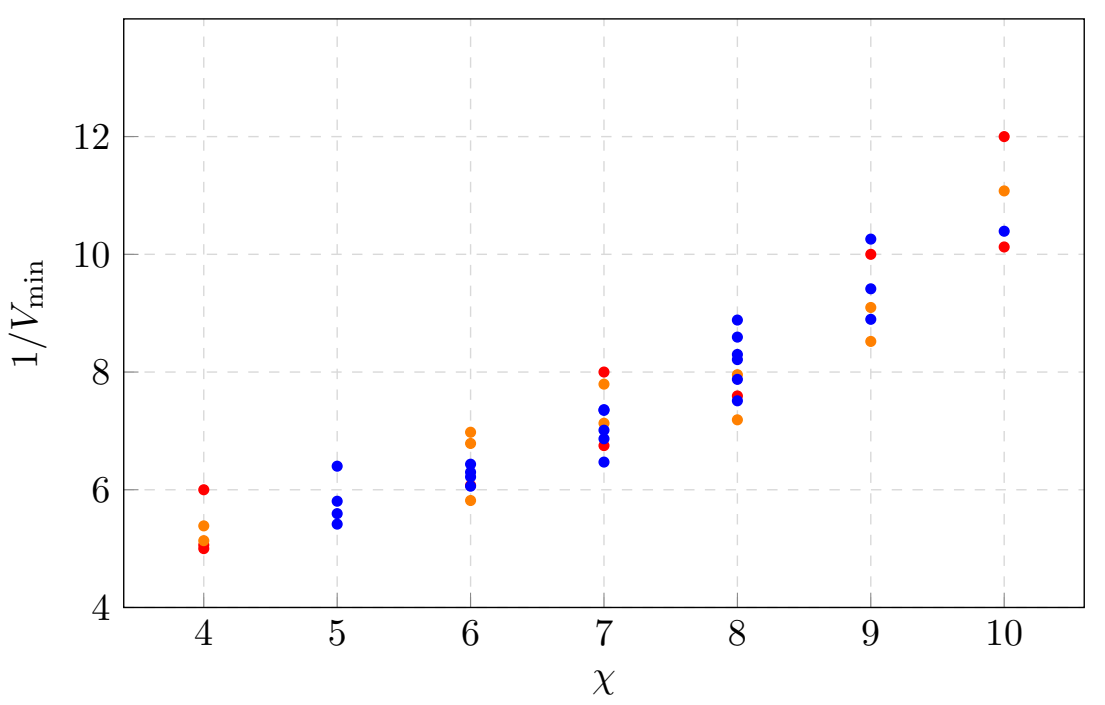

Figure 6. The red points correspond to regular Sasaki-Einstein manifolds while the quasi-regular ones are in orange. We omit the first-grade points in the plot.

As a matter of fact, all the minimized volume functions of Sasaki-Einstein manifolds $Y$ are algebraic. When $V_{\text {min }} \in \mathbb{Q}, Y$ is said to be regular. If $V_{\min } \in \mathbb{Q}(\sqrt{c})(c \in \mathbb{N})$, viz, quadratic irrationals, then $Y$ is quasi-regular. In figure 6 , we plot the $1 / V_{\min }$ against $\chi$, with regular and quasi-regular $Y$ 's highlighted.

Fine-tuning R-charges. There are 22 Sasaki-Einstein manifolds that are neither regular nor quasi-regular. As a result, the expressions/equations to solve the R-charges in the main text are also in decimals which are not exact. Nevertheless, we can express the exact R-charges in terms of roots of some polynomials. As an example, the volume function discussed in section 4.8 is reproduced here:

$$
V=\frac{2\left(2 b_{1}+b_{2}+15\right)}{\left(b_{2}+3\right)\left(-b_{1}+b_{2}-3\right)\left(b_{1}+b_{2}+3\right)\left(b_{1}+2 b_{2}-6\right)} .
$$

This reaches the minimum when $b_{1}=x_{0}$ and $b_{2}=y_{0}$, where $x_{0}$ is the only positive root of the equation

$$
-1296-192 x+100 x^{2}+21 x^{3}+x^{4}=0
$$


and

$$
y_{0}=\frac{-86345699328+342641664 x_{0}+4796983296 x_{0}^{2}+342641664 x_{0}^{3}}{20558499840+1713208320 x_{0}} .
$$

Then the R-charges of the GLSM fields should satisfy

$$
\begin{gathered}
351+432 p_{3} p_{4}-216 p_{3} p_{4}^{2}+171 y_{0}-3 y_{0}^{2}-7 y_{0}^{3}-90 x_{0}-12 x_{0} y_{0}+6 x_{0} y_{0}^{2}+12 x_{0}^{2}+4 x_{0}^{2} y_{0} \\
+\frac{-6561-2916 y_{0}+162 y_{0}^{2}+108 y_{0}^{3}-9 y_{0}^{4}}{15+2 x_{0}+y_{0}}=p_{2}^{2}\left(432 p_{3}+108 p_{4}\right)+p_{2}\left(-864 p_{3}+432 p_{3}^{2}\right. \\
\left.-216 p_{4}+432 p_{3} p_{4}+108 p_{4}^{2}\right)
\end{gathered}
$$

constrained by $\sum_{i=1}^{4} p_{i}=2$ and $0<p_{i}<2$, with others vanishing.

\section{Higgsing the parent theory}

The Higgs mechanism states that by turning on a non-zero vev of a bifundamental and integrating out the quadratic mass terms in superpotential, we would get a theory with a different moduli space. This corresponds to removal of an edge in the brane tiling and merger of two gauge nodes in the quiver. In terms of toric diagrams, it is easy to identify the parent theories by blowing up/down points. For instance, (3.37) is the parent of all the triangles and the pentagon (5.87) is the parent of all the hexagons here. As a simple example, we consider higgsing (3.10) to the theory of $\mathrm{dP}_{0}$ :

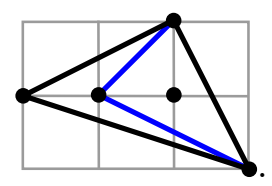

The superpotential of the parent theory is

$$
\begin{aligned}
W= & X_{12}^{1} X_{25} X_{51}^{2}+X_{12}^{2} X_{23}^{1} X_{31}+X_{23}^{2} X_{34}^{1} X_{42}+X_{34}^{2} X_{45}^{1} X_{53}+X_{45}^{2} X_{51}^{1} X_{14} \\
& -X_{12}^{2} X_{25} X_{51}^{1}-X_{12}^{1} X_{23}^{2} X_{31}-X_{23}^{1} X_{34}^{2} X_{42}-X_{34}^{1} X_{45}^{2} X_{53}-X_{45}^{1} X_{51}^{2} X_{14} .
\end{aligned}
$$

We first give a non-zero vev to $X_{53}$, viz, $\left\langle X_{53}\right\rangle=1$ :

$$
\begin{aligned}
W= & X_{12}^{1} X_{25} X_{51}^{2}+X_{12}^{2} X_{23}^{1} X_{31}+X_{23}^{2} X_{34}^{1} X_{42}+X_{34}^{2} X_{45}^{1}+X_{45}^{2} X_{51}^{1} X_{14} \\
& -X_{12}^{2} X_{25} X_{51}^{1}-X_{12}^{1} X_{23}^{2} X_{31}-X_{23}^{1} X_{34}^{2} X_{42}-X_{34}^{1} X_{45}^{2}-X_{45}^{1} X_{51}^{2} X_{14} .
\end{aligned}
$$

Integrating our the quadratic terms yields

$$
\begin{aligned}
W= & X_{12}^{1} X_{23}^{3} X_{31}^{2}+X_{12}^{2} X_{23}^{1} X_{31}^{3}+X_{42} X_{23}^{2} X_{31}^{1} X_{14} \\
& -X_{12}^{2} X_{23}^{3} X_{31}^{1}-X_{12}^{1} X_{23}^{2} X_{31}^{3}-X_{42} X_{23}^{1} X_{31}^{2} X_{14} .
\end{aligned}
$$

Finally, by turning on a vev of $X_{42}$ such that $\left\langle X_{42}\right\rangle=1$, the superpotential becomes

$$
\begin{aligned}
W= & X_{12}^{1} X_{23}^{3} X_{31}^{2}+X_{12}^{2} X_{23}^{1} X_{31}^{3}+X_{23}^{2} X_{31}^{1} X_{12}^{3} \\
& -X_{12}^{2} X_{23}^{3} X_{31}^{1}-X_{12}^{1} X_{23}^{2} X_{31}^{3}-X_{23}^{1} X_{31}^{2} X_{12}^{3},
\end{aligned}
$$


which is exactly the superpotential of the $\mathrm{dP}_{0}$ theory. In terms of quivers, we have
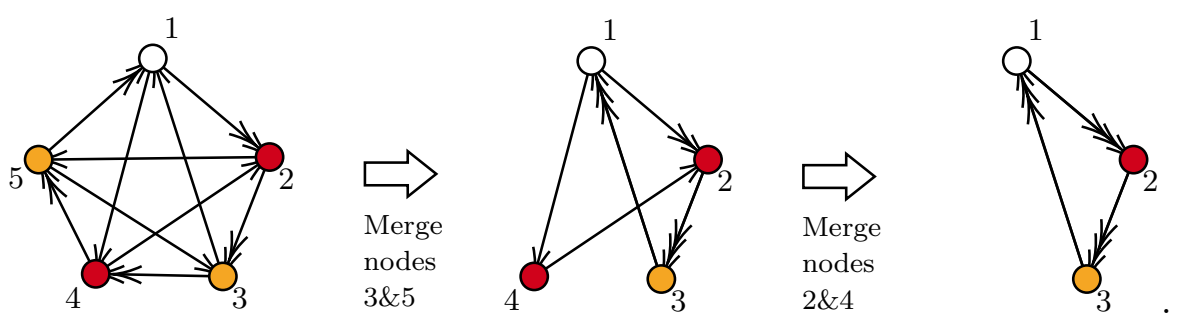

As a matter of fact, the 45 polygons can be higgsed from a same parent theory. This theory can be $\mathbb{C}^{3} /\left(\mathbb{Z}_{6} \times \mathbb{Z}_{6}\right)(1,0,5)(0,1,5)$ such that there is only one corresponding quiver in the toric phase. It is a huge quiver with 36 nodes and 108 bifundamentals. The Rcharges of the bifundamentals are all $2 / 3$, and hence the three GLSM fields corresponding to the extremal points all have R-charge $2 / 3$, with others vanishing. If we only want the minimal parent toric diagram, then we would have $\mathcal{C} /\left(\mathbb{Z}_{6} \times \mathbb{Z}_{2}\right)(1,0,0,5)(0,1,1,0)$.

\section{More toric phases}

Here, we list the toric quivers (other than those appeared in section 3-6) and the corresponding superpotentials for some of the polytopes. Notice that we are not listing all the toric quivers here (especially for those in section 5-6) as this is exhaustive. These quivers can be obtained via Seiberg duality as discussed in section 2.2. All the triangles only have one quiver in the toric phase (up to permutation equivalence). Different quivers of all the quadrilaterals are tabulated in table 4.

\begin{tabular}{|c|c|}
\hline Polytope-Phase & Quiver \& Superpotential \\
\hline 7 -2 (section 4.2) & $W=X_{12}^{1} X_{26} X_{61}^{2}+X_{12}^{2} X_{23}^{1} X_{31}-X_{15}^{1} X_{54}^{2} X_{41}+X_{15}^{2} X_{56} X_{61}^{1}$ \\
& $-X_{35}^{2} X_{54}^{1} X_{43}+X_{35}^{1} X_{52} X_{23}^{2}-X_{12}^{2} X_{26} X_{61}^{1}-X_{12}^{1} X_{23}^{2} X_{31}$ \\
& $+X_{15}^{1} X_{54}^{2} X_{41}-X_{15}^{1} X_{56} X_{61}^{2}+X_{35}^{1} X_{54}^{2} X_{43}-X_{35}^{2} X_{52} X_{23}^{1}$ \\
\hline
\end{tabular}




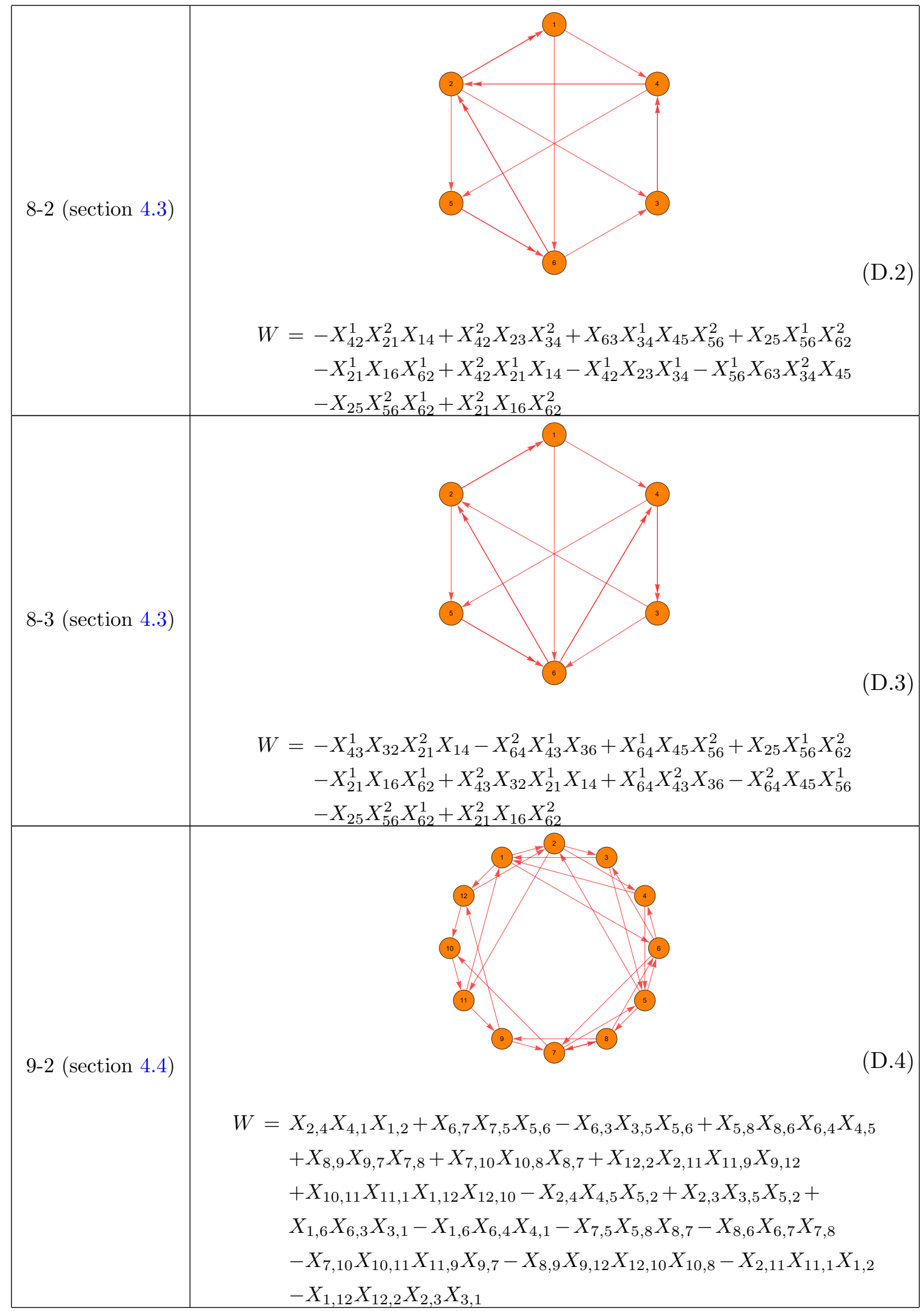




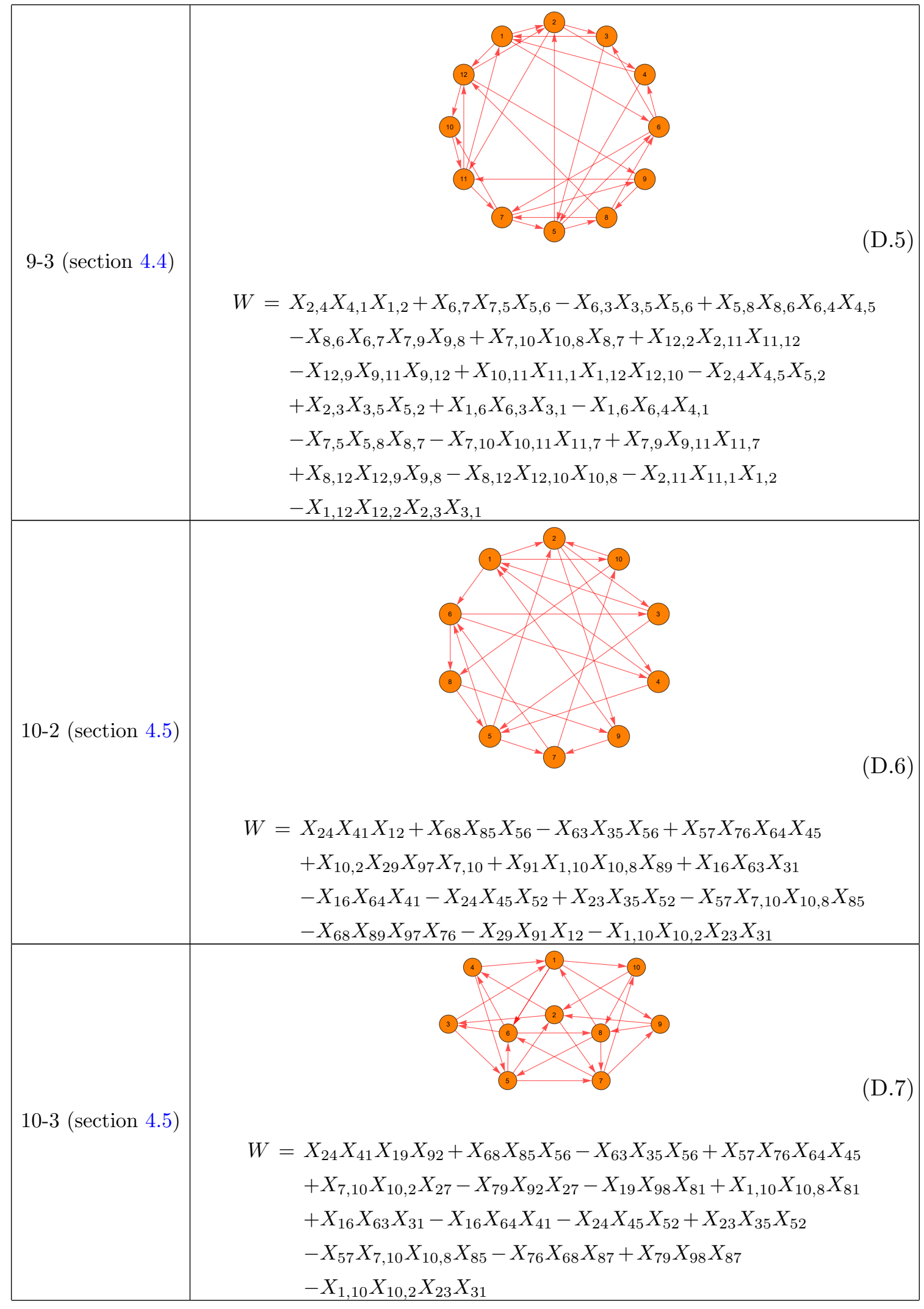




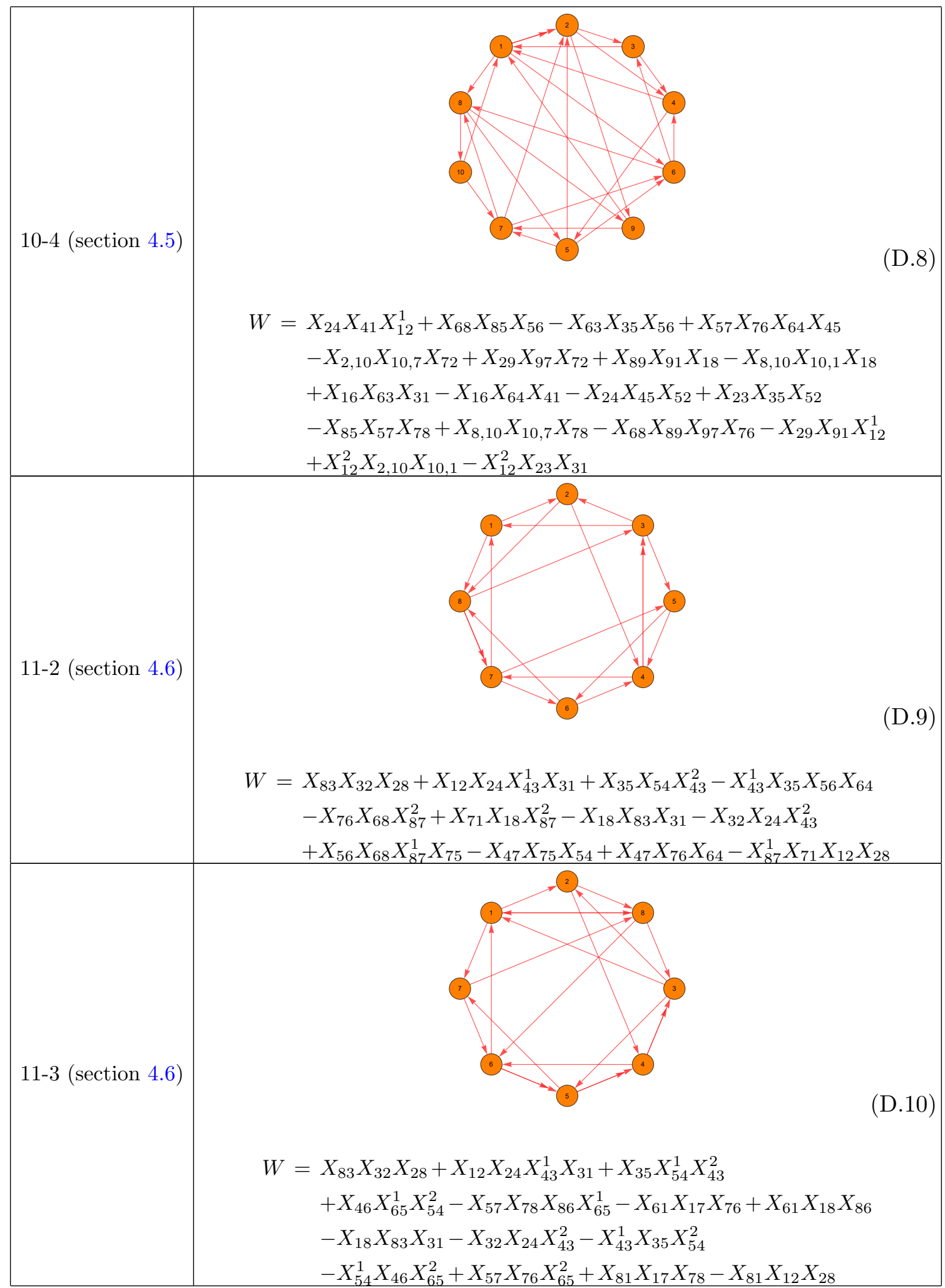




12-2 (section 4.7)




13-3 (section 4.8)




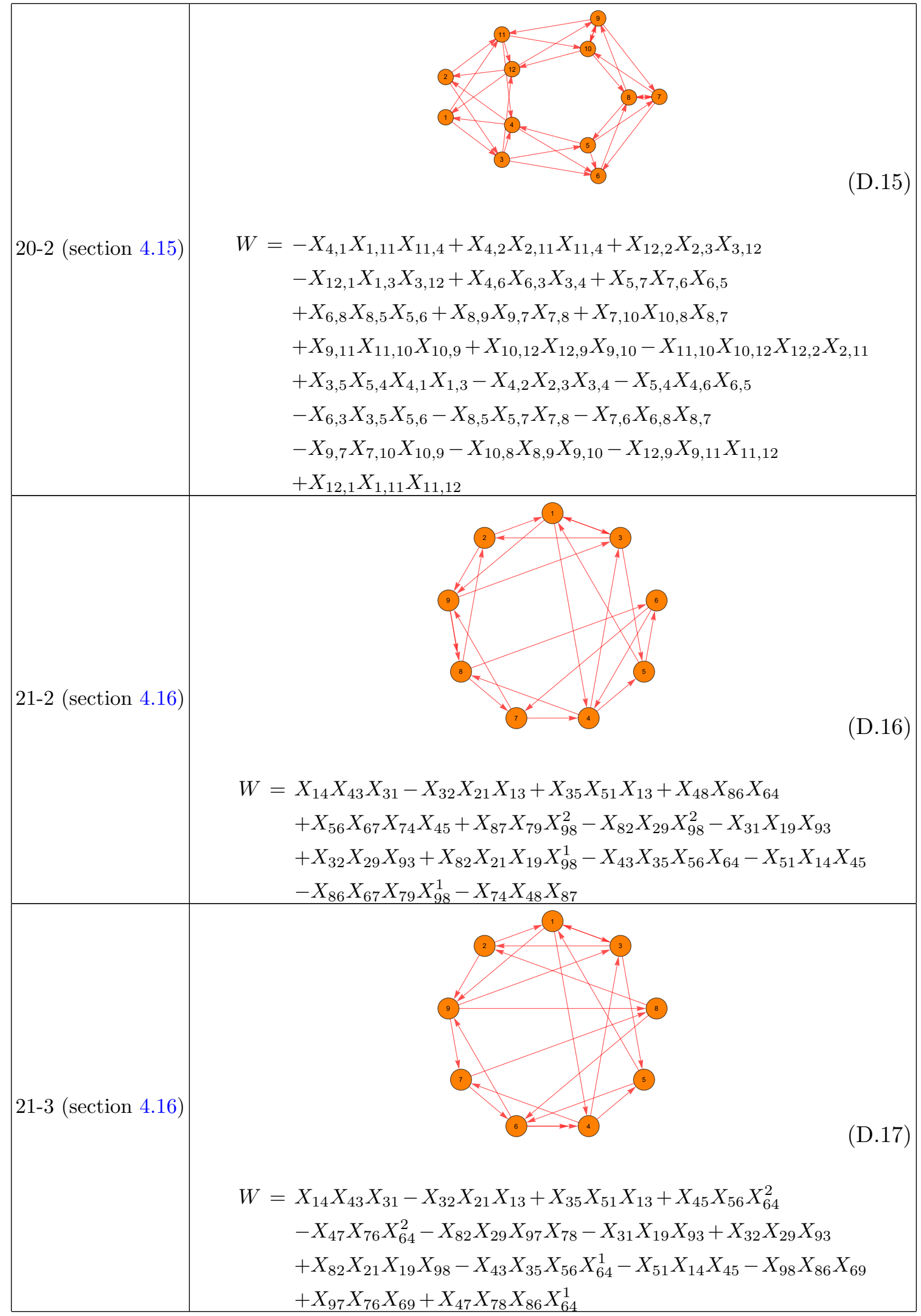




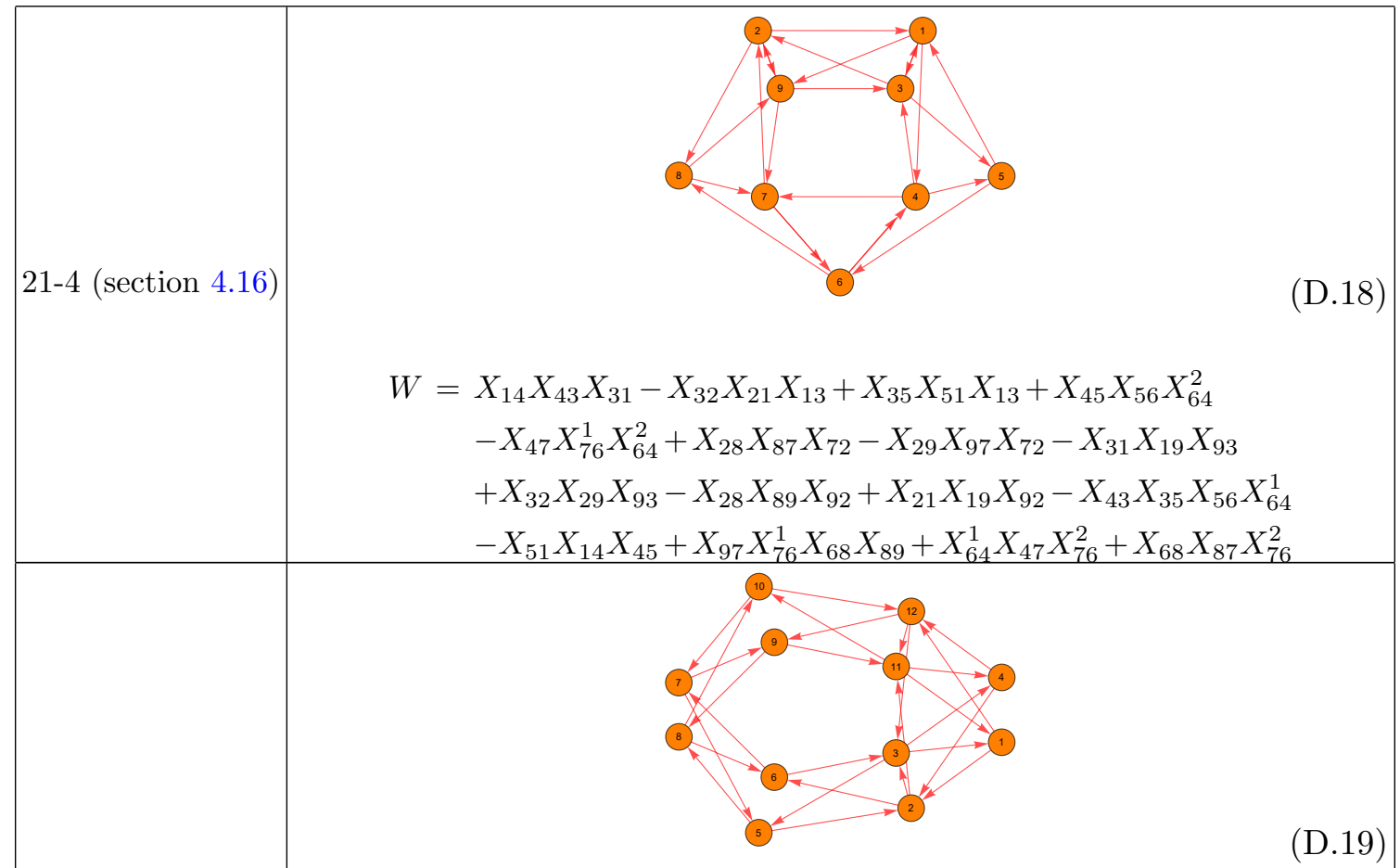

22-2 (section 4.17)

$$
\begin{aligned}
W= & -X_{12,11} X_{11,1} X_{1,12}+X_{12,11} X_{11,10} X_{10,12}+X_{11,4} X_{4,12} X_{12,9} X_{9,11} \\
& +X_{3,5} X_{5,2} X_{2,3}-X_{3,1} X_{1,2} X_{2,3}+X_{2,6} X_{6,3} X_{3,4} X_{4,2} \\
& +X_{8,10} X_{10,7} X_{7,5} X_{5,8}+X_{7,9} X_{9,8} X_{8,6} X_{6,7}+X_{11,1} X_{1,2} X_{2,11} \\
& -X_{11,4} X_{4,2} X_{2,11}-X_{3,4} X_{4,12} X_{12,3}+X_{3,1} X_{1,12} X_{12,3} \\
& -X_{5,2} X_{2,6} X_{6,7} X_{7,5}-X_{6,3} X_{3,5} X_{5,8} X_{8,6}-X_{10,7} X_{7,9} X_{9,11} X_{11,10} \\
& -X_{9,8} X_{8,10} X_{10,12} X_{12,9}
\end{aligned}
$$

$22-3$ (section 4.17)

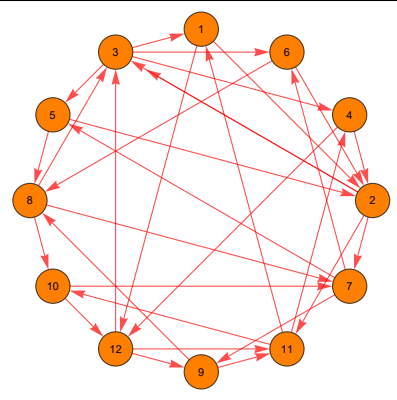

$$
\begin{aligned}
W= & -X_{12,11} X_{11,1} X_{1,12}+X_{12,11} X_{11,10} X_{10,12}+X_{11,4} X_{4,12} X_{12,9} X_{9,11} \\
& +X_{3,5} X_{5,2} X_{2,3}^{1}-X_{3,1} X_{1,2} X_{2,3}^{1}+X_{3,4} X_{4,2} X_{2,3}^{2} \\
& -X_{3,6} X_{6,2} X_{2,3}^{2}+X_{8,10} X_{10,7} X_{7,5} X_{5,8}+X_{7,9} X_{9,8} X_{8,7} \\
& -X_{7,6} X_{6,8} X_{8,7}+X_{11,1} X_{1,2} X_{2,11}-X_{11,4} X_{4,2} X_{2,11} \\
& -X_{3,4} X_{4,12} X_{12,3}+X_{3,1} X_{1,12} X_{12,3}-X_{7,5} X_{5,2} X_{2,7} \\
& +X_{7,6} X_{6,2} X_{2,7}+X_{3,6} X_{6,8} X_{8,3}-X_{3,5} X_{5,8} X_{8,3} \\
& -X_{10,7} X_{7,9} X_{9,11} X_{11,10}-X_{9,8} X_{8,10} X_{10,12} X_{12,9}
\end{aligned}
$$




22-4 (section 4.17)




22-6 (section 4.17)




23-2 (section 4.18)

Table 4. The remaining toric phases of quadrilaterals. 
We also give three examples of pentagons in table $5-7$.

Quiver \& Superpotential




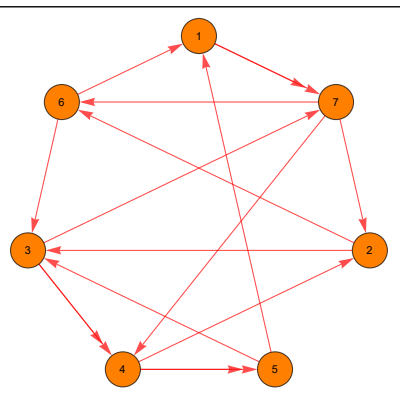

(D.31)

$W=X_{17}^{1} X_{74} X_{45}^{2} X_{51}+X_{53} X_{34}^{2} X_{45}^{1}+X_{37} X_{76} X_{63}-X_{37} X_{72} X_{23}^{1}$ $-X_{26} X_{61} X_{17}^{1} X_{72}+X_{17}^{2} X_{76} X_{61}-X_{17}^{2} X_{74} X_{45}^{1} X_{51}-X_{45}^{2} X_{53} X_{34}^{1}$ $-X_{34}^{2} X_{42} X_{23}^{1}+X_{26} X_{63} X_{34}^{1} X_{42}$

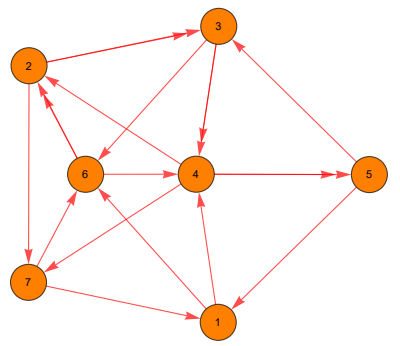

$$
\begin{aligned}
W= & -X_{14} X_{47} X_{71}+X_{14} X_{45}^{2} X_{51}+X_{53} X_{34}^{2} X_{45}^{1}+X_{34}^{1} X_{42} X_{23}^{2} \\
& +X_{62}^{2} X_{23}^{1} X_{36}-X_{62}^{2} X_{27} X_{76}-X_{45}^{1} X_{51} X_{16} X_{64}+X_{47} X_{76} X_{64} \\
& -X_{45}^{2} X_{53} X_{34}^{1}-X_{34}^{2} X_{42} X_{23}^{1}-X_{23}^{2} X_{36} X_{62}^{1}+X_{27} X_{71} X_{16} X_{62}^{1}
\end{aligned}
$$

Table 5. The remaining toric phases of (5.2) in section 5.1.

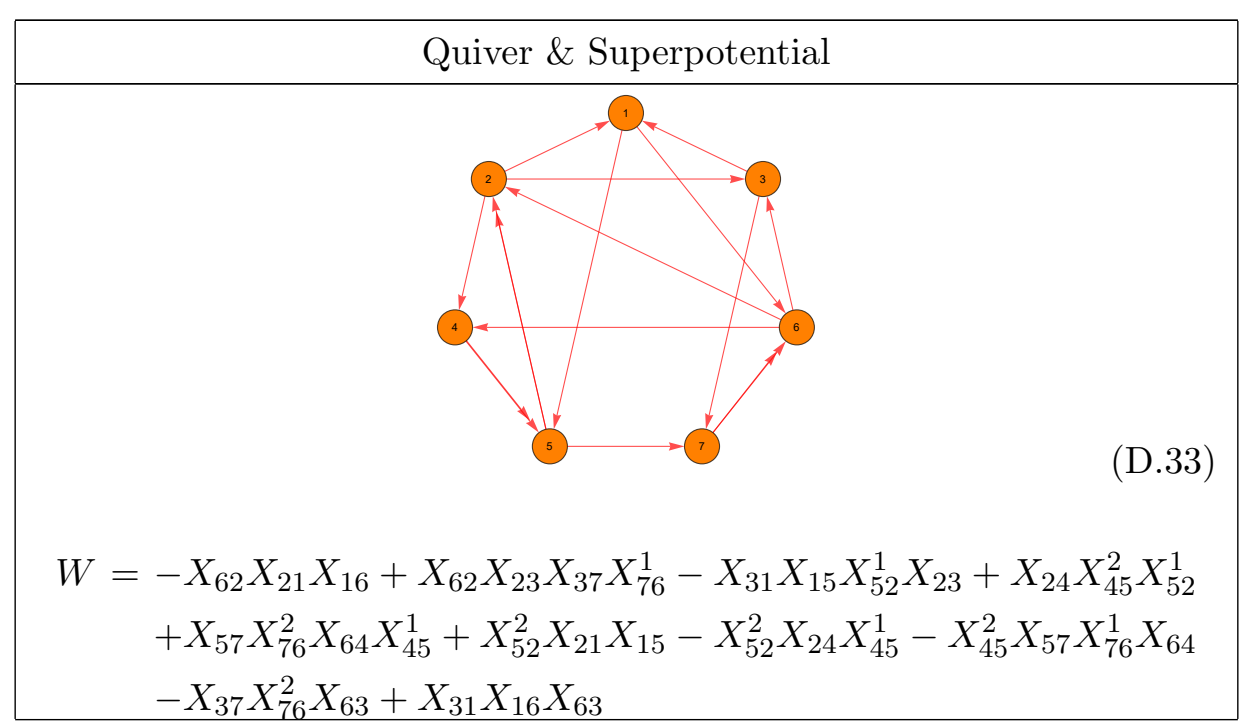




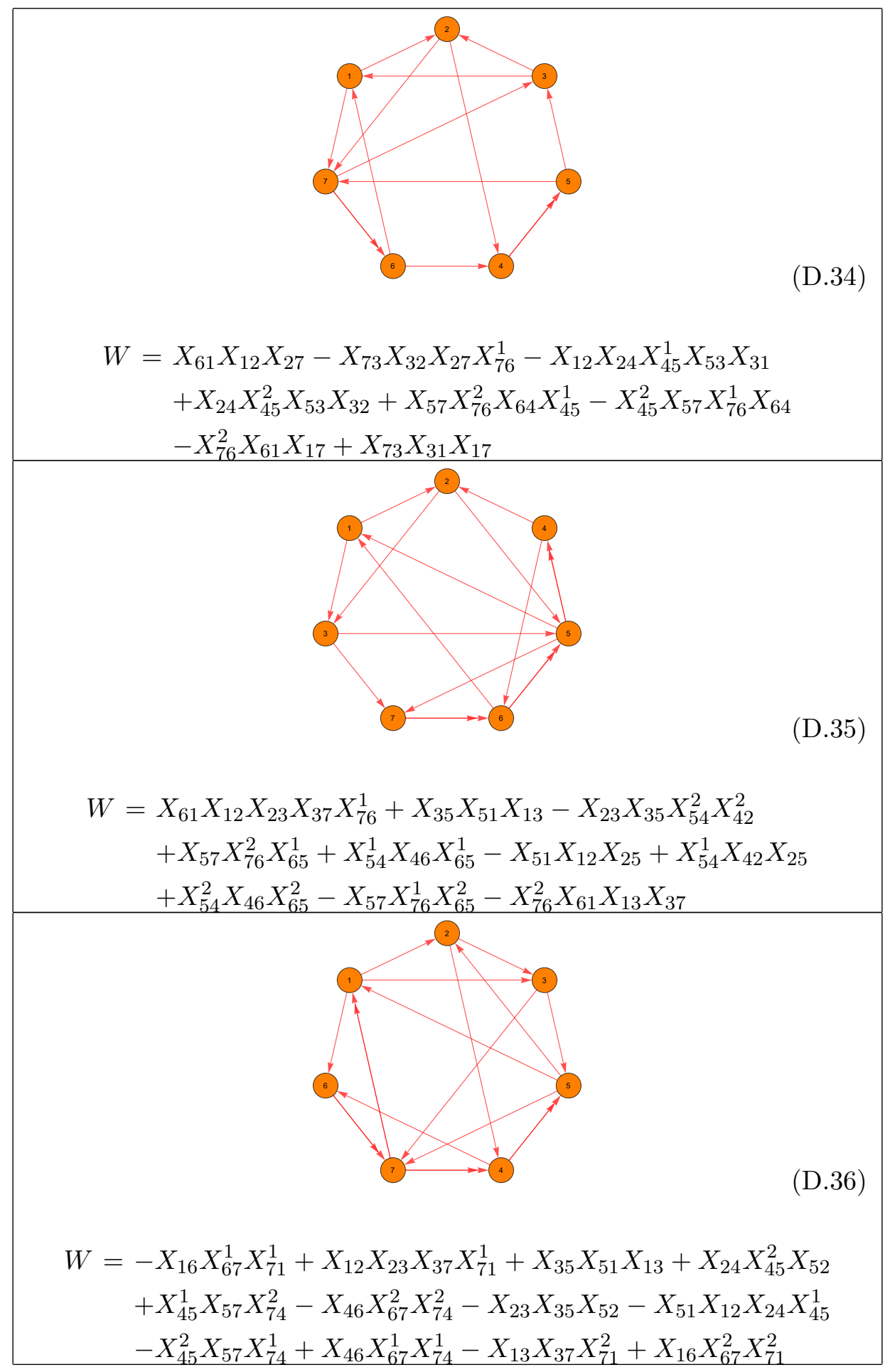




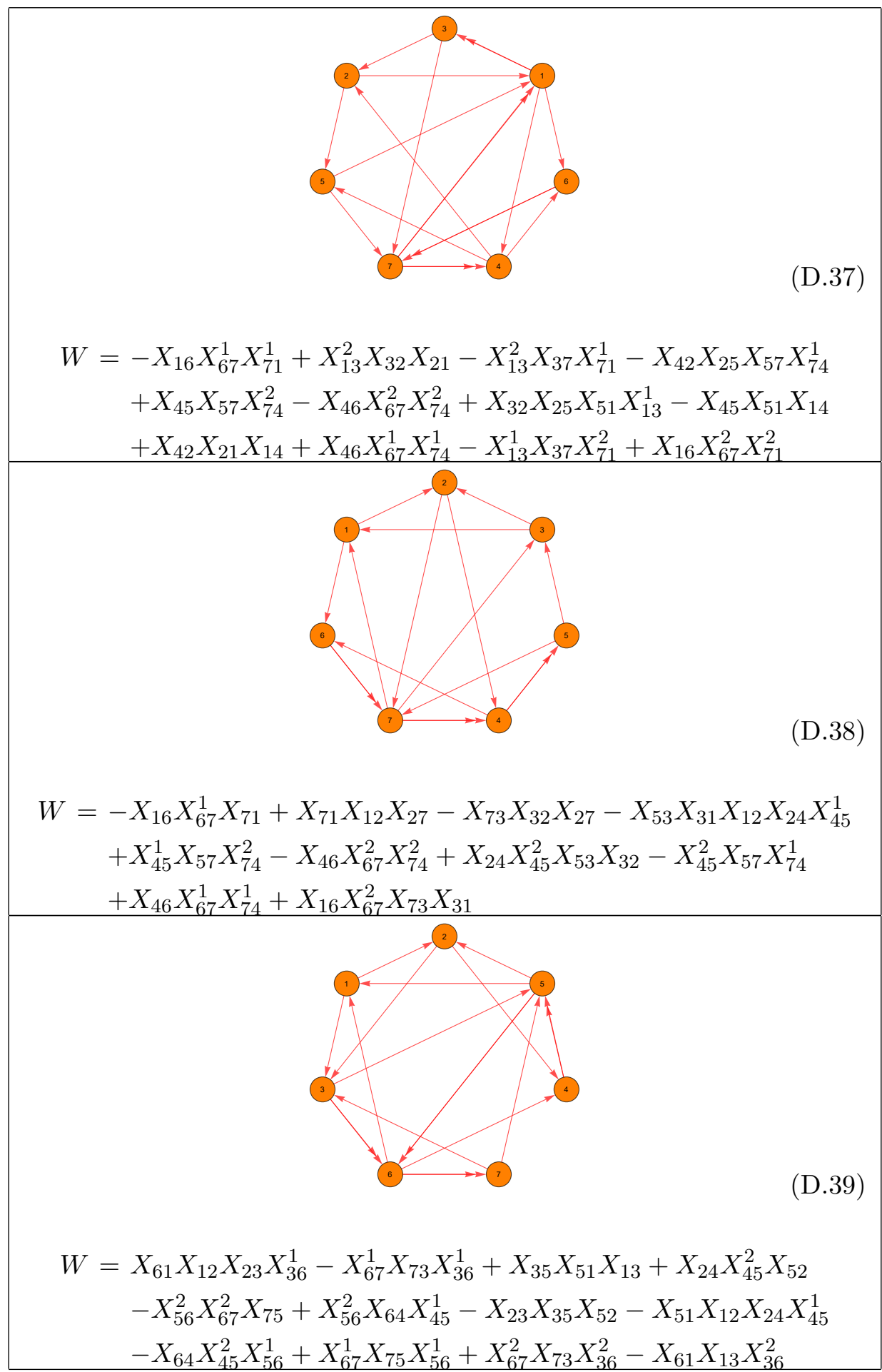




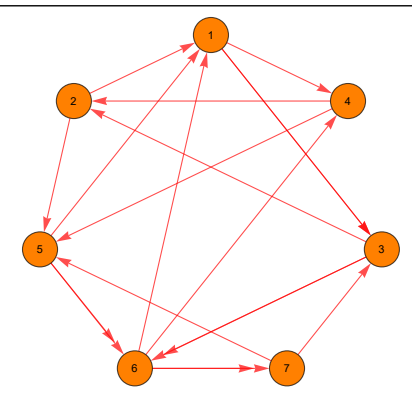

(D.40)

$$
\begin{aligned}
W= & X_{36}^{1} X_{61} X_{13}^{2}-X_{32} X_{21} X_{13}^{2}-X_{67}^{1} X_{73} X_{36}^{1}-X_{42} X_{25} X_{56}^{1} X_{64} \\
& -X_{56}^{2} X_{67}^{2} X_{75}+X_{56}^{2} X_{64} X_{45}^{1}+X_{32} X_{25} X_{51} X_{13}^{1}-X_{45}^{1} X_{51} X_{14} \\
& +X_{42} X_{21} X_{14}+X_{67}^{1} X_{75} X_{56}^{1}+X_{67}^{2} X_{73} X_{36}^{2}-X_{61} X_{13}^{1} X_{36}^{2}
\end{aligned}
$$

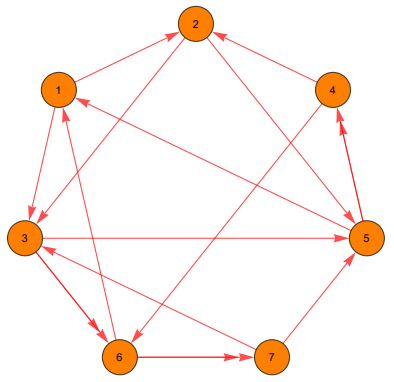

$$
\begin{aligned}
W= & X_{61} X_{12} X_{23} X_{36}^{1}-X_{67}^{1} X_{73} X_{36}^{1}+X_{35} X_{51} X_{13}+X_{23} X_{35} X_{54}^{2} X_{42} \\
& -X_{54} X_{46} X_{67}^{2} X_{75}-X_{51} X_{12} X_{25}+X_{54} X_{42}^{1} X_{25}+X_{67}^{1} X_{75} X_{54} X_{46} \\
& +X_{67}^{2} X_{73} X_{36}^{2}-X_{61} X_{13} X_{36}^{2}
\end{aligned}
$$

Table 6. The remaining toric phases of (5.12) in section 5.2.

Quiver \& Superpotential
$W=-X_{32} X_{21}^{1} X_{13}+X_{35} X_{51} X_{13}+X_{56} X_{67} X_{74} X_{45}+X_{48} X_{86} X_{64}$
$+X_{87} X_{71} X_{18}^{2}-X_{82} X_{21}^{2} X_{18}^{2}+X_{14} X_{43} X_{32} X_{21}^{2}-X_{43} X_{35} X_{56} X_{64}$
$-X_{51} X_{14} X_{45}-X_{86} X_{67} X_{71} X_{18}^{1}+X_{82} X_{21}^{1} X_{18}^{1}-X_{74} X_{48} X_{87}$




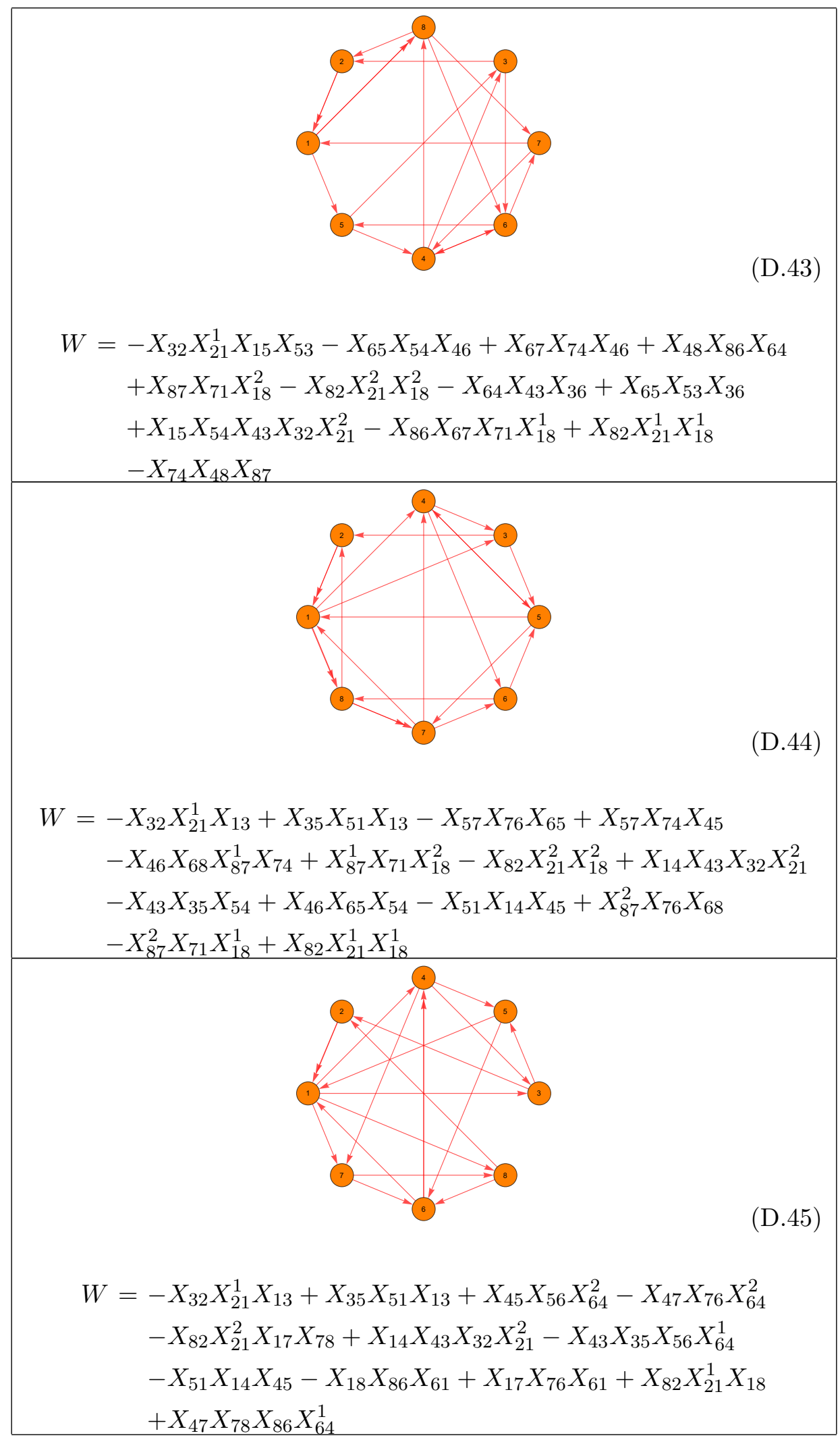




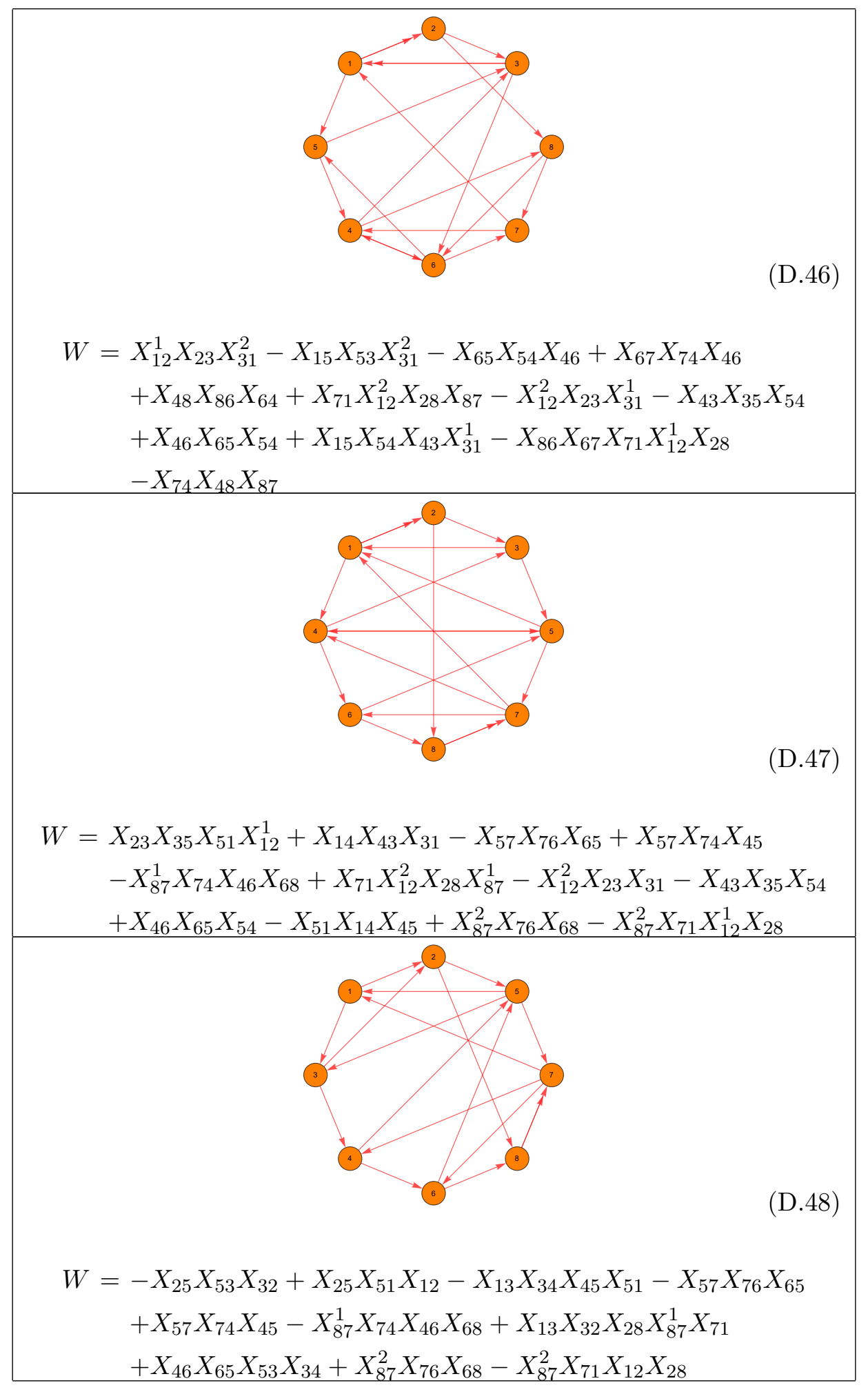




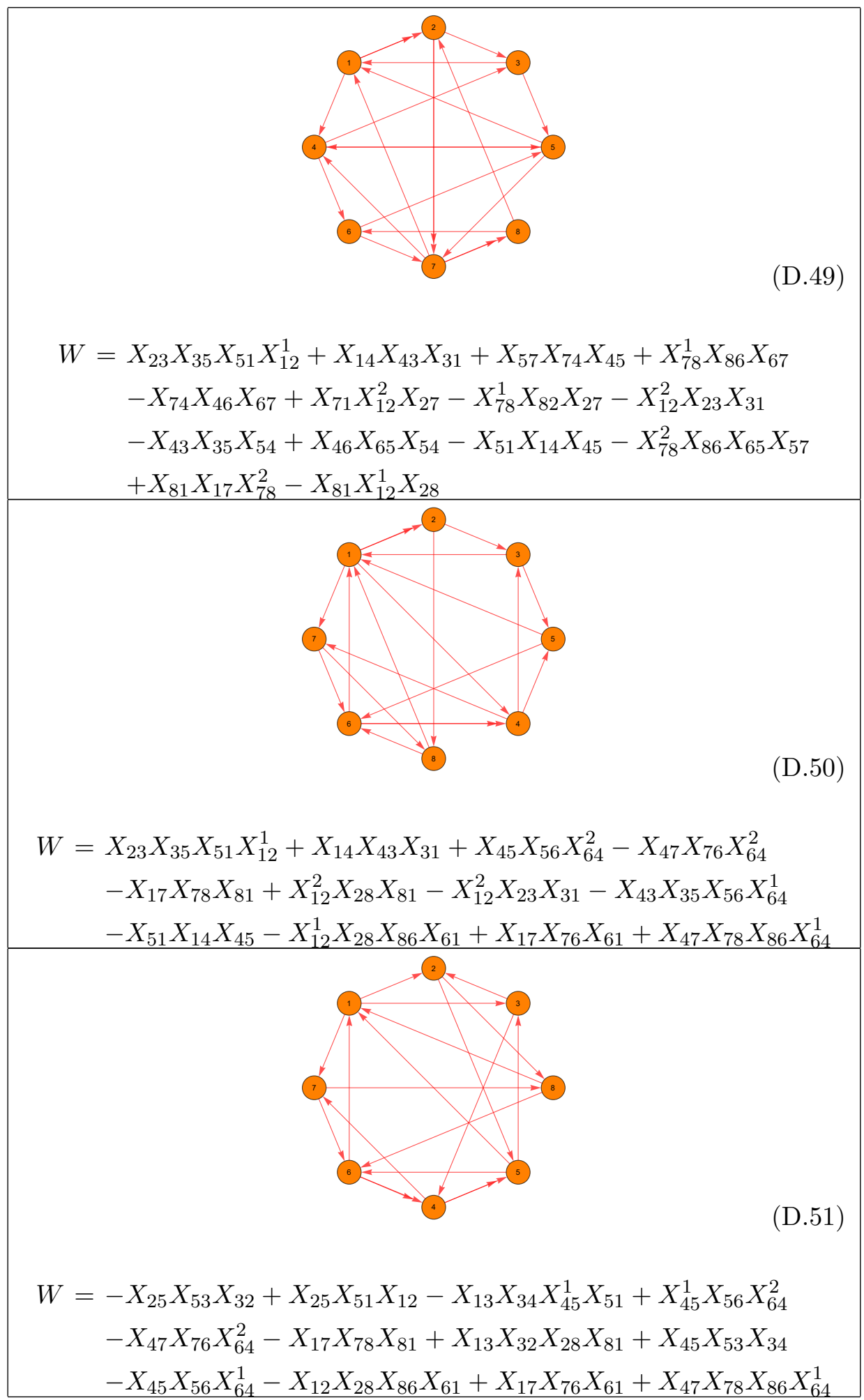




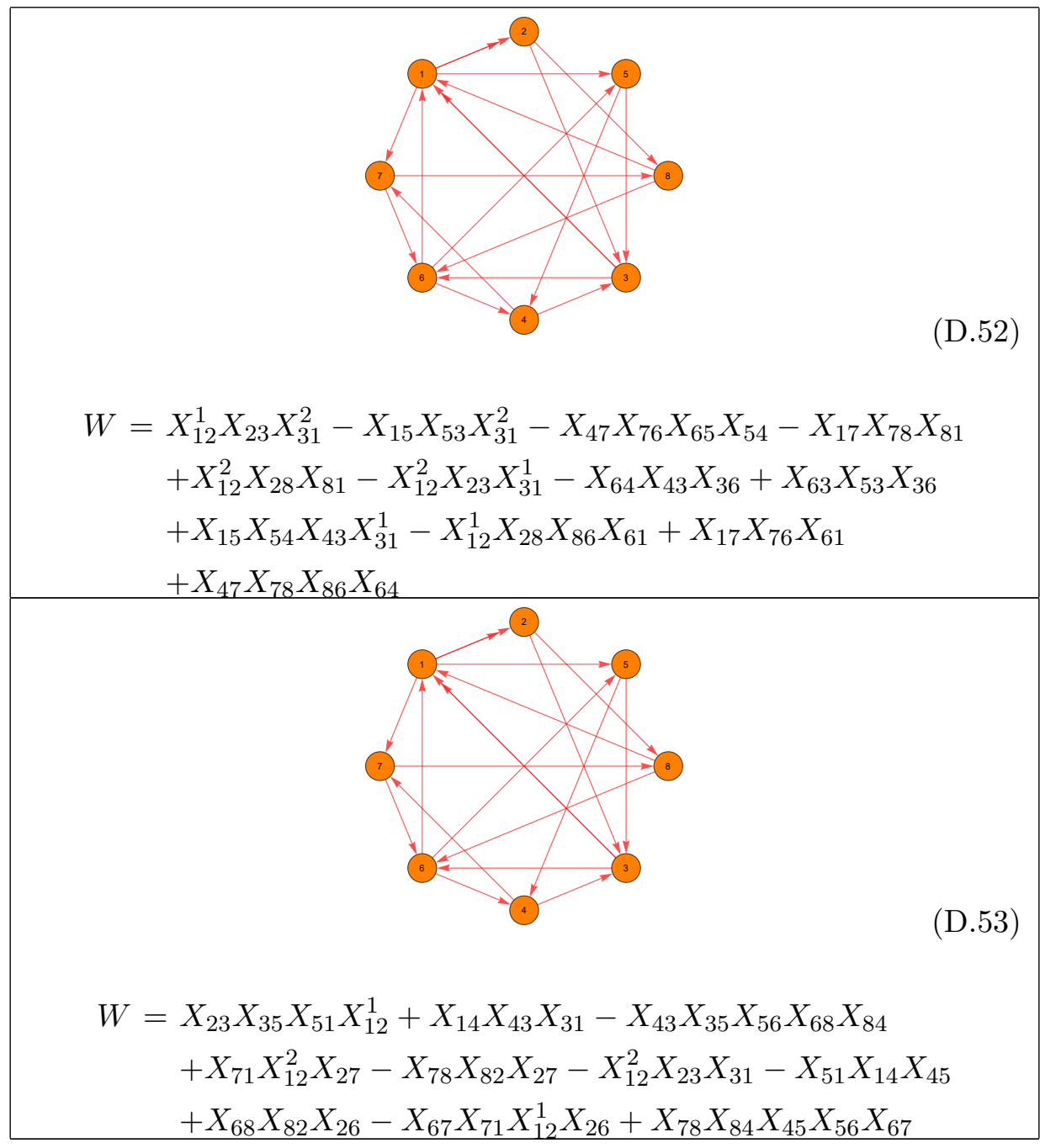

Table 7. The remaining toric phases of (5.22) in section 5.3.

Open Access. This article is distributed under the terms of the Creative Commons Attribution License (CC-BY 4.0), which permits any use, distribution and reproduction in any medium, provided the original author(s) and source are credited.

\section{References}

[1] B. Feng, A. Hanany and Y.-H. He, D-brane gauge theories from toric singularities and toric duality, Nucl. Phys. B 595 (2001) 165 [hep-th/0003085] [INSPIRE].

[2] B. Feng, Y.-H. He and F. Lam, On correspondences between toric singularities and $(p, q)$ webs, Nucl. Phys. B 701 (2004) 334 [hep-th/0403133] [InSPIRE].

[3] D.R. Gulotta, Properly ordered dimers, R-charges and an efficient inverse algorithm, JHEP 10 (2008) 014 [arXiv:0807.3012] [INSPIRE].

[4] B. Feng, A. Hanany, Y.-H. He and A.M. Uranga, Toric duality as Seiberg duality and brane diamonds, JHEP 12 (2001) 035 [hep-th/0109063] [INSPIRE]. 
[5] J.M. Maldacena, The large $N$ limit of superconformal field theories and supergravity, Int. J. Theor. Phys. 38 (1999) 1113 [hep-th/9711200] [INSPIRE].

[6] B.S. Acharya, J.M. Figueroa-O'Farrill, C.M. Hull and B.J. Spence, Branes at conical singularities and holography, Adv. Theor. Math. Phys. 2 (1999) 1249 [hep-th/9808014] [INSPIRE].

[7] D.R. Morrison and M. Plesser, Nonspherical horizons. 1, Adv. Theor. Math. Phys. 3 (1999) 1 [hep-th/9810201] [INSPIRE].

[8] A. Hanany and K.D. Kennaway, Dimer models and toric diagrams, hep-th/0503149 [INSPIRE].

[9] S. Franco, A. Hanany, K.D. Kennaway, D. Vegh and B. Wecht, Brane dimers and quiver gauge theories, JHEP 01 (2006) 096 [hep-th/0504110] [INSPIRE].

[10] B. Feng, Y.-H. He, K.D. Kennaway and C. Vafa, Dimer models from mirror symmetry and quivering amoebae, Adv. Theor. Math. Phys. 12 (2008) 489 [hep-th/0511287] [InSPIRE].

[11] R. Kenyon, Local statistics of lattice dimers, Ann. Inst. H. Poincaré B 33 (1997) 591 [math.C0/0105054].

[12] R. Kenyon, An introduction to the dimer model, math.c0/0310326.

[13] Y.-H. He, R.-K. Seong and S.-T. Yau, Calabi-Yau volumes and reflexive polytopes, Commun. Math. Phys. 361 (2018) 155 [arXiv:1704.03462] [InSPIRE].

[14] O. Aharony, O. Bergman, D.L. Jafferis and J. Maldacena, $N=6$ superconformal Chern-Simons-matter theories, M2-branes and their gravity duals, JHEP 10 (2008) 091 [arXiv:0806.1218] [INSPIRE].

[15] J. Bagger and N. Lambert, Modeling multiple M2's, Phys. Rev. D 75 (2007) 045020 [hep-th/0611108] [INSPIRE].

[16] A. Gustavsson, Algebraic structures on parallel M2-branes, Nucl. Phys. B 811 (2009) 66 [arXiv:0709.1260] [INSPIRE].

[17] A. Hanany and A. Zaffaroni, Tilings, Chern-Simons theories and M2 branes, JHEP 10 (2008) 111 [arXiv:0808.1244] [INSPIRE].

[18] A. Hanany, D. Vegh and A. Zaffaroni, Brane tilings and M2 branes, JHEP 03 (2009) 012 [arXiv:0809.1440] [INSPIRE].

[19] S. Franco, D. Ghim, S. Lee, R.-K. Seong and D. Yokoyama, $2 d(0,2)$ quiver gauge theories and D-branes, JHEP 09 (2015) 072 [arXiv: 1506.03818] [INSPIRE].

[20] S. Franco, S. Lee and R.-K. Seong, Brane brick models, toric Calabi-Yau 4-folds and 2d $(0,2)$ quivers, JHEP 02 (2016) 047 [arXiv: 1510.01744] [INSPIRE].

[21] S. Franco, S. Lee, R.-K. Seong and C. Vafa, Brane brick models in the mirror, JHEP 02 (2017) 106 [arXiv:1609.01723] [INSPIRE].

[22] S. Franco, S. Lee and R.-K. Seong, Brane brick models and 2d (0,2) triality, JHEP 05 (2016) 020 [arXiv: 1602.01834] [INSPIRE].

[23] A. Gadde, S. Gukov and P. Putrov, (0,2) trialities, JHEP 03 (2014) 076 [arXiv: 1310.0818] [INSPIRE].

[24] S. Franco, S. Lee, R.-K. Seong and C. Vafa, Quadrality for supersymmetric matrix models, JHEP 07 (2017) 053 [arXiv: 1612.06859] [INSPIRE]. 
[25] A. Hanany and R.-K. Seong, Brane tilings and reflexive polygons, Fortsch. Phys. 60 (2012) 695 [arXiv: 1201.2614] [INSPIRE].

[26] A. Hanany and R.-K. Seong, Brane tilings and specular duality, JHEP 08 (2012) 107 [arXiv: 1206.2386] [INSPIRE].

[27] X. Wei and R. Ding, Lattice polygons with two interior lattice points, Math. Notes 91 (2012) 868.

[28] J. Park, R. Rabadán and A.M. Uranga, Orientifolding the conifold, Nucl. Phys. B 570 (2000) 38 [hep-th/9907086] [INSPIRE].

[29] A.M. Uranga, Brane configurations for branes at conifolds, JHEP 01 (1999) 022 [hep-th/9811004] [INSPIRE].

[30] J.P. Gauntlett, D. Martelli, J. Sparks and D. Waldram, Supersymmetric AdS $S_{5}$ solutions of M-theory, Class. Quant. Grav. 21 (2004) 4335 [hep-th/0402153] [InSPIRE].

[31] J.P. Gauntlett, D. Martelli, J. Sparks and D. Waldram, Sasaki-Einstein metrics on $S^{2} \times S^{3}$, Adv. Theor. Math. Phys. 8 (2004) 711 [hep-th/0403002] [InSPIRE].

[32] S. Benvenuti, S. Franco, A. Hanany, D. Martelli and J. Sparks, An infinite family of superconformal quiver gauge theories with Sasaki-Einstein duals, JHEP 06 (2005) 064 [hep-th/0411264] [INSPIRE].

[33] S. Benvenuti, A. Hanany and P. Kazakopoulos, The toric phases of the $Y^{p, q}$ quivers, JHEP 07 (2005) 021 [hep-th/0412279] [INSPIRE].

[34] S. Franco, A. Hanany, D. Martelli, J. Sparks, D. Vegh and B. Wecht, Gauge theories from toric geometry and brane tilings, JHEP 01 (2006) 128 [hep-th/0505211] [INSPIRE].

[35] A. Hanany, P. Kazakopoulos and B. Wecht, A new infinite class of quiver gauge theories, JHEP 08 (2005) 054 [hep-th/0503177] [INSPIRE].

[36] B. Feng, A. Hanany and Y.-H. He, Phase structure of D-brane gauge theories and toric duality, JHEP 08 (2001) 040 [hep-th/0104259] [INSPIRE].

[37] B. Feng, S. Franco, A. Hanany and Y.-H. He, UnHiggsing the del Pezzo, JHEP 08 (2003) 058 [hep-th/0209228] [INSPIRE].

[38] B. Feng, S. Franco, A. Hanany and Y.-H. He, Symmetries of toric duality, JHEP 12 (2002) 076 [hep-th/0205144] [INSPIRE].

[39] A. Hanany and R.-K. Seong, Symmetries of Abelian orbifolds, JHEP 01 (2011) 027 [arXiv: 1009.3017] [INSPIRE].

[40] J. Davey, A. Hanany and R.-K. Seong, Counting orbifolds, JHEP 06 (2010) 010 [arXiv: 1002.3609] [INSPIRE].

[41] J. Davey, A. Hanany and J. Pasukonis, On the classification of brane tilings, JHEP 01 (2010) 078 [arXiv:0909.2868] [INSPIRE].

[42] S. Franco, Y.-H. He, C. Sun and Y. Xiao, A comprehensive survey of brane tilings, Int. J. Mod. Phys. A 32 (2017) 1750142 [arXiv:1702.03958] [InSPIRE].

[43] C. Closset, M. Del Zotto and V. Saxena, Five-dimensional SCFTs and gauge theory phases: an M-theory/type IIA perspective, SciPost Phys. 6 (2019) 052 [arXiv:1812.10451] [INSPIRE].

[44] C. Closset and M. Del Zotto, On 5d SCFTs and their BPS quivers. Part I: B-branes and brane tilings, arXiv:1912.13502 [INSPIRE].

[45] V. Saxena, Rank-two 5d SCFTs from M-theory at isolated toric singularities: a systematic study, JHEP 20 (2020) 198 [arXiv:1911.09574] [INSPIRE]. 
[46] W. Fulton, Introduction to toric varieties, Annals of mathematics studies, Princeton University Press, Princeton, NJ, U.S.A. (1993).

[47] D. Cox, J. Little and H. Schenck, Toric varieties, Graduate studies in mathematics, American Mathematical Soc., U.S.A. (2011).

[48] G.S. Guralnik, C.R. Hagen and T.W.B. Kibble, Global conservation laws and massless particles, Phys. Rev. Lett. 13 (1964) 585 [InSPIRE].

[49] P.W. Higgs, Broken symmetries and the masses of gauge bosons, Phys. Rev. Lett. 13 (1964) 508 [INSPIRE].

[50] F. Englert and R. Brout, Broken symmetry and the mass of gauge vector mesons, Phys. Rev. Lett. 13 (1964) 321 [INSPIRE].

[51] M. Yamazaki, Brane tilings and their applications, Fortsch. Phys. 56 (2008) 555 [arXiv: 0803.4474] [INSPIRE].

[52] J. Bao, Y.-H. He, E. Hirst and S. Pietromonaco, Lectures on the Calabi-Yau landscape, arXiv: 2001.01212 [INSPIRE].

[53] E. Witten, Phases of $N=2$ theories in two-dimensions, AMS/IP Stud. Adv. Math. 1 (1996) 143 [hep-th/9301042] [inSPIRE].

[54] N. Seiberg, Electric-magnetic duality in supersymmetric non-Abelian gauge theories, Nucl. Phys. B 435 (1995) 129 [hep-th/9411149] [INSPIRE].

[55] C.E. Beasley and M. Plesser, Toric duality is Seiberg duality, JHEP 12 (2001) 001 [hep-th/0109053] [INSPIRE].

[56] G. Musiker and C. Stump, A compendium on the cluster algebra and quiver package in sage, arXiv:1102.4844.

[57] A. Hanany, Y.-H. He, V. Jejjala, J. Pasukonis, S. Ramgoolam and D. Rodriguez-Gomez, Invariants of toric Seiberg duality, Int. J. Mod. Phys. A 27 (2012) 1250002 [arXiv: 1107.4101] [INSPIRE].

[58] S. Franco, A. Hanany, Y.-H. He and P. Kazakopoulos, Duality walls, duality trees and fractional branes, hep-th/0306092 [INSPIRE].

[59] A. Hanany, Y.-H. He, C. Sun and S. Sypsas, Superconformal block quivers, duality trees and Diophantine equations, JHEP 11 (2013) 017 [arXiv:1211.6111] [INSPIRE].

[60] D. Forcella, A. Hanany, Y.-H. He and A. Zaffaroni, The master space of $N=1$ gauge theories, JHEP 08 (2008) 012 [arXiv:0801.1585] [INSPIRE].

[61] D. Forcella, A. Hanany, Y.-H. He and A. Zaffaroni, Mastering the master space, Lett. Math. Phys. 85 (2008) 163 [arXiv:0801.3477] [InSPIRE].

[62] D. Martelli, J. Sparks and S.-T. Yau, The geometric dual of a-maximisation for toric Sasaki-Einstein manifolds, Commun. Math. Phys. 268 (2006) 39 [hep-th/0503183] [INSPIRE].

[63] D. Martelli, J. Sparks and S.-T. Yau, Sasaki-Einstein manifolds and volume minimisation, Commun. Math. Phys. 280 (2008) 611 [hep-th/0603021] [InSPIRE].

[64] S. Benvenuti, B. Feng, A. Hanany and Y.-H. He, Counting BPS operators in gauge theories: quivers, Syzygies and Plethystics, JHEP 11 (2007) 050 [hep-th/0608050] [INSPIRE].

[65] S.S. Gubser, Einstein manifolds and conformal field theories, Phys. Rev. D 59 (1999) 025006 [hep-th/9807164] [INSPIRE]. 
[66] K.A. Intriligator and B. Wecht, The exact superconformal $R$ symmetry maximizes a, Nucl. Phys. B 667 (2003) 183 [hep-th/0304128] [INSPIRE].

[67] A. Butti and A. Zaffaroni, R-charges from toric diagrams and the equivalence of a-maximization and Z-minimization, JHEP 11 (2005) 019 [hep-th/0506232] [INSPIRE].

[68] A. Butti and A. Zaffaroni, From toric geometry to quiver gauge theory: the equivalence of a-maximization and Z-minimization, hep-th/0512240 [INSPIRE].

[69] M. Henningson and K. Skenderis, The holographic Weyl anomaly, JHEP 07 (1998) 023 [hep-th/9806087] [INSPIRE].

[70] D.Z. Freedman, S.S. Gubser, K. Pilch and N.P. Warner, Renormalization group flows from holography supersymmetry and a $c$ theorem, Adv. Theor. Math. Phys. 3 (1999) 363 [hep-th/9904017] [INSPIRE].

[71] R. Altman, J. Gray, Y.-H. He, V. Jejjala and B.D. Nelson, A Calabi-Yau database: threefolds constructed from the Kreuzer-Skarke list, JHEP 02 (2015) 158 [arXiv:1411.1418] [INSPIRE].

[72] B. Nill, Gorenstein toric Fano varieties, Manuscripta Math. 116 (2005) 183 [math. AG/0405448].

[73] S. Cabrera and A. Hanany, Branes and the Kraft-Procesi transition, JHEP 11 (2016) 175 [arXiv: 1609.07798] [INSPIRE].

[74] S. Cabrera and A. Hanany, Branes and the Kraft-Procesi transition: classical case, JHEP 04 (2018) 127 [arXiv: 1711.02378] [INSPIRE].

[75] A. Bourget et al., The Higgs mechanism - Hasse diagrams for symplectic singularities, JHEP 01 (2020) 157 [arXiv: 1908. 04245] [INSPIRE].

[76] A. Bourget, S. Cabrera, J.F. Grimminger, A. Hanany and Z. Zhong, Brane webs and magnetic quivers for SQCD, JHEP 03 (2020) 176 [arXiv: 1909.00667] [INSPIRE].

[77] S. Cabrera, A. Hanany and M. Sperling, Magnetic quivers, Higgs branches and $6 d N=(1,0)$ theories - orthogonal and symplectic gauge groups, JHEP 02 (2020) 184 [arXiv: 1912.02773] [INSPIRE].

[78] J.F. Grimminger and A. Hanany, Hasse diagrams for $3 d N=4$ quiver gauge theories inversion and the full moduli space, arXiv:2004.01675 [INSPIRE].

[79] G. Balletti and A.M. Kasprzyk, Three-dimensional lattice polytopes with two interior lattice points, arXiv:1612.08918.

[80] V.V. Batyrev, Toroidal Fano 3-folds, Math. USSR Izv. 19 (1982) 13.

[81] V.V. Batyrev and L.A. Borisov, On Calabi-Yau complete intersections in toric varieties, alg-geom/9412017 [INSPIRE].

[82] M. Kreuzer and H. Skarke, On the classification of reflexive polyhedra, Commun. Math. Phys. 185 (1997) 495 [hep-th/9512204] [INSPIRE].

[83] M. Kreuzer and H. Skarke, Classification of reflexive polyhedra in three-dimensions, Adv. Theor. Math. Phys. 2 (1998) 853 [hep-th/9805190] [InSPIRE].

[84] M. Kreuzer and H. Skarke, Complete classification of reflexive polyhedra in four-dimensions, Adv. Theor. Math. Phys. 4 (2002) 1209 [hep-th/0002240] [INSPIRE].

[85] M. Kreuzer and H. Skarke, PALP: a Package for Analyzing Lattice Polytopes with applications to toric geometry, Comput. Phys. Commun. 157 (2004) 87 [math.NA/0204356] [INSPIRE]. 